Proceedings of RIKEN BNL Research Center Workshop Volume 60

\title{
Lattice QCD at Finite \\ Temperature and Density
}

February 8-12, 2004

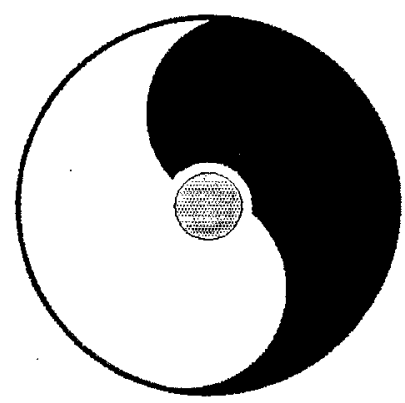

Organizers

T. Blum, M. Creutz, and P. Petreczky

RIKEN BNL Research Center

Building 510A, Brookhaven National Laboratory, Upton, NY 11973-5000, USA 


\section{DISCLAIMER}

This report was prepared as an account of work sponsored by an agency of the United States Government. Neither the United States Government nor any agency thereof, nor any of their employees, nor any of their contractors, subcontractors or their employees, makes any warranty, express or implied, or assumes any legal liability or responsibility for the accuracy, completeness, or any third party's use or the results of such use of any information, apparatus, product, or process disclosed, or represents that its use would not infringe privately owned rights. Reference herein to any specific commercial product, process, or service by trade name, trademark, manufacturer, or otherwise, does not necessarily constitute or imply its endorsement, recommendation, or favoring by the United States Government or any agency thereof or its contractors or subcontractors. The views and opinions of authors expressed herein do not necessarily state or reflect those of the United States Government or any agency thereof.

\section{FOR UNCLASSIFIED, UNLIMITED STI PRODUCTS}

Available electronically at-

\section{OSTI:}

http://www.osti.gov/bridge

Available for a processing fee to U.S. Department of Energy and it contractors, in paper from-

U.S. Department of Energy

Office of Scientific and Technical Information

P.O. Box 62

Oak Ridge, TN 37831

(865) 576-8401

Facsimile: (865) 576-5728

E-mail: reports@adonis.osti.gov

\section{National Technical Information Service (NTIS):}

Available for sale to the public from-

U.S. Department of Commerce

National Technical Information Service

5285 Port Royal Road

Springfield, VA 22131

(800) 553-6847

Facsimile: (703) 605-6900

Online ordering: http://www.ntis.gov/ordering.htm 
The RIKEN BNL Research Center (RBRC) was established in April 1997 at Brookhaven National Laboratory. It is funded by the "Rikagaku Kenkyusho" (RIKEN, The Institute of Physical and Chemical Research) of Japan. The Center is dedicated to the study of strong interactions, including spin physics, lattice QCD, and RHIC physics through the nurturing of a new generation of young physicists.

During the first year, the Center had only a Theory Group. In the second year, an Experimental Group was also established at the Center. At present, there are three Fellows and seven Research Associates in these two groups. During the third year, we started a new Tenure Track Strong Interaction Theory RHIC Physics Fellow Program, with six positions in the first academic year, 1999-2000. This program had increased to include ten theorists and one experimentalist in academic year, 2001-2002. With five fellows having already graduated, the program presently has ten theorists and three experimentalists. Five of the ten RHIC Physics Fellows have been awarded/offered tenured positions, and this will be their final year in the program.

Beginning in 2001 a new RIKEN Spin Program (RSP) category was implemented at RBRC. These appointments are joint positions of RBRC and RIKEN and include the following positions in theory and experiment: RSP Researchers, RSP Research Associates, and Young Researchers, who are mentored by RBRC Researchers. A number of RIKEN Jr. Research Associates and Visiting Scientists also contribute to the physics program at the Center.

RBRC also has an active workshop program on strong interaction physics with each workshop focused on a specific physics problem. Each workshop speaker is encouraged to select a few of the most important transparencies from his or her presentation, accompanied by a page of explanation. This material is collected at the end of the workshop by the organizer to form proceedings, which can therefore be available within a short time. To date there are fiftynine proceeding volumes available.

The construction of a 0.6 teraflops parallel processor, dedicated to lattice QCD, begun at the Center on February 19, 1998, was completed on August 28, 1998. A 10 teraflops QCDOC computer in under development and expected to be completed in JFY 2004.

\section{N. P. Samios, Director January 2004}

*Work performed under the auspices of U.S.D.O.E. Contract No. DE-AC02-98CH10886. 


\section{CONTENTS}

Preface to the Series

Introduction

N. Samios, M. Creutz, T. Blum, P. Petreczk............................ i

QCD thermodynamics from lattice calculations

F. Karsch .............................................................. 1

Domain wall fermion thermodynamics

R. Mawhinney .................................................... $\quad 7$

Phase diagram of finite temperature QCD with improved staggered fermions

U. M. Heller

Lattice QCD at finite T and $\mu$, phase diagram and the critical point

Z. Fodor

Taylor expansion at non-zero density and the chiral critical end-point at 3 flavor QCD

C. Schmidt

QCD thermodynamics from imaginary $\mu$

O. Philipsen

The QCD equation of state at finite $\mathrm{T}$ and $\mu$ : lattice result

S. Katz

QCD thermodynamics at non-zero $\mu$

S. Ejiri

QCD at zero baryon density

$P$. de Forcrand

Potentials and free energies of static quarks

O. Philipsen 
New developments in the field of Polyakov loop correlation functions

Heavy quark free energies and the renormalized Polyakov in full QCD

O. Kaczmarek

Static quark-antiquark potentials at finite temperature with Asqtad improved fermions

K. Petrov

Mesonic spectral functions at finite (high) temperature

M. Asakawa

Charmonia in finite temperature quenched QCD

S. Datta

Properties of the glueball at finite temperature from anisotropic lattice QCD

N. Ishii

Finite temperature meson correlations of light quarks

E. Laermann

Meson correlators in lattice $\mathrm{QCD}$ at finite temperature

H. Matsufuru

Temporal and spatial hadron corrrelators in finite temperature QCD

S. Gupta

Lattice simulations at the Fermi surface

S. Hands

Order from disorder in lattice QCD with baryons

B. Bringoltz

The Quark-gluon plasma at strong (QCD) and very strong (CFT) coupling

E. Shuryak

Renormalized Polyakov loops and matrix models 
Viscosity in heavy-ion collisions

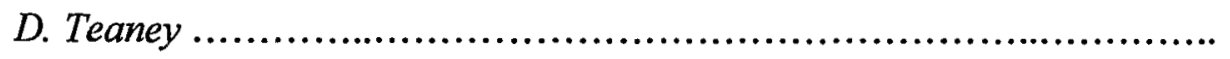

Pressure of hot $\mathrm{QCD}$ - is $\mathrm{g}^{6}$ and beyond calculable?

K. Kajantie

Mesonic screening masses at high temperature

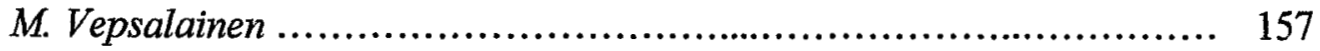

Gluon screening masses

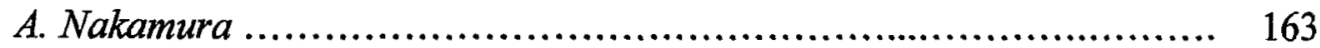

List of Registered Participants............................................. 167

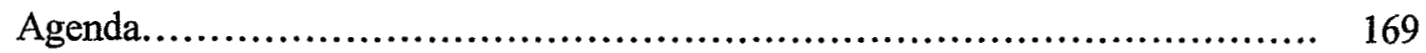

Additional RIKEN BNL Research Center Proceeding Volumes.................. 173

Contact Information 


\title{
Lattice QCD at finite temperature and density
}

\author{
Introduction and overview
}

With the operation of the RHIC heavy ion program, the theoretical understanding of QCD at finite temperature and density has become increasingly important. Though QCD at finite temperature has been extensively studied using lattice Monte-Carlo simulations over the past twenty years, most physical questions relevant for RHIC (and future) heavy ion experiments remain open.

In lattice QCD at finite temperature and density there have been at least two major advances in recent years. First, for the first time calculations of real time quantities, like meson spectral functions have become available. Second, the lattice study of the QCD phase diagram and equation of state have been extended to finite baryon density by several groups. Both issues were extensively discussed in the course of the workshop. A real highlight was the study of the QCD phase diagram in $(T, \mu)$-plane by $\mathrm{Z}$. Fodor and $\mathrm{S}$. Katz and the determination of the critical end-point for the physical value of the pion mass. This was the first time such lattice calculations at the physical pion mass have been performed. Results by Z. Fodor and S. Katz were obtained using a multi-parameter re-weighting method. Other determinations of the critical end point were also presented, in particular using a Taylor expansion around $\mu=0$ (Bielefeld group, Ejiri et al.) and using analytic continuation from imaginary chemical potential (Ph. de Forcrand and $O$. Philipsen). The result based on Taylor expansion agrees within errors with the new prediction of Z. Fodor and S. Katz, while methods based on analytic continuation still predict a higher value for the critical baryon density.

Most of the thermodynamics studies in full QCD (including those presented at this workshop) have been performed using quite coarse lattices, $a=0.2-0.3 \mathrm{fm}$. Therefore one may worry about cutoff effects in different thermodynamic quantities, like the transition temperature $T_{t r}$. At the workshop U. Heller presented a study of the transition temperature for three different lattice spacings and performed a continuum extrapolation of $T_{t r}$ for the first time. 
Lattice calculations of the meson spectral functions were presented by M. Asakawa, S. Datta, E. Laermann and H. Matsufuru. These show that charmonia ground states $\left(\eta_{c}\right.$ and $\left.J / \psi\right)$ continue to exist in the plasma at least up to a temperature of $1.7 T_{t r}$. At what temperature charmonia states cease to exist is not yet clear. Calculations presented by M. Asakawa show dissolution of the $J / \psi$ at $T=1.7 T_{t r}$, while the analysis presented $\mathrm{H}$. Matsufuru provided evidence that ground state charmonia still exist at this temperature. S. Datta argued that the ground state charmonia is likely to dissolve only for temperatures $T>2.25 T_{t r}$, while the $P$-states are dissociated at $1.1 T_{t r}$. It is also very interesting that, even in the case of light quarks, meson spectral functions show a resonance-like structure in the plasma phase (talk by $E$. Laermann). Finally attempts to calculate transport properties in the Quark Gluon Plasma were presented by S. Gupta.

The workshop devoted special attention to the finite temperature modification of inter-quark forces and color screening, another area where considerable progress has been made in recent years (talks by O. Kaczmarek, K. Petrov, O. Philipsen and F. Zantow). Many other new theoretical developments which cannot be discussed here were also presented on the workshop. Altogether the workshop was a great success, for which we thank all the participants.

We would like to thank Prof. T.D. Lee and the RIKEN BNL center for providing the opportunity and support to organize this workshop. We also thank Brookhaven National Laboratory and the U.S. Department of Energy for financial support. Finally, sincere thanks go to Pamela Esposito for her invaluable help in organizing and running the workshop as well as to Frithjof Karsch for many useful comments and suggestions concerning the scientific program.

T. Blum, M. Creutz and P. Petreczky

Brookhaven National Laboratory, February, 2004 


\title{
QCD Thermodynamics from Lattice Calculations
}

\author{
Frithjof Karsch
}

Fakultät für Physik, Universität Bielefeld, D-33615 Bielefeld, Germany

\section{SUMMARY}

We discuss lattice results on the QCD transition from hadronic matter to the quark gluon plasma and point out where lattice calculations can provide input to the interpretation of heavy ion collisions.

We emphasize the importance of lattice calculations for QCD phenomenology of the Quark Gluon Plasma and stress the need for careful control over lattice cut-off and finite volume effects to extrapolate lattice thermodynamics performed in a finite volume to the continuum and thermodynamic limit.

In particular, we discuss the role of resonances for the occurrence of the transition to the quark-gluon plasma in hot and dense matter. Properties of a hadronic resonance gas are compared to lattice results on the equation of state at zero as well as non-zero baryon chemical potential. We also show that the quark mass dependence of the transition temperature can be understood in terms of lines of constant energy density in a resonance gas. 


\section{Critical behavior in hot and dense matter: phase diagram}

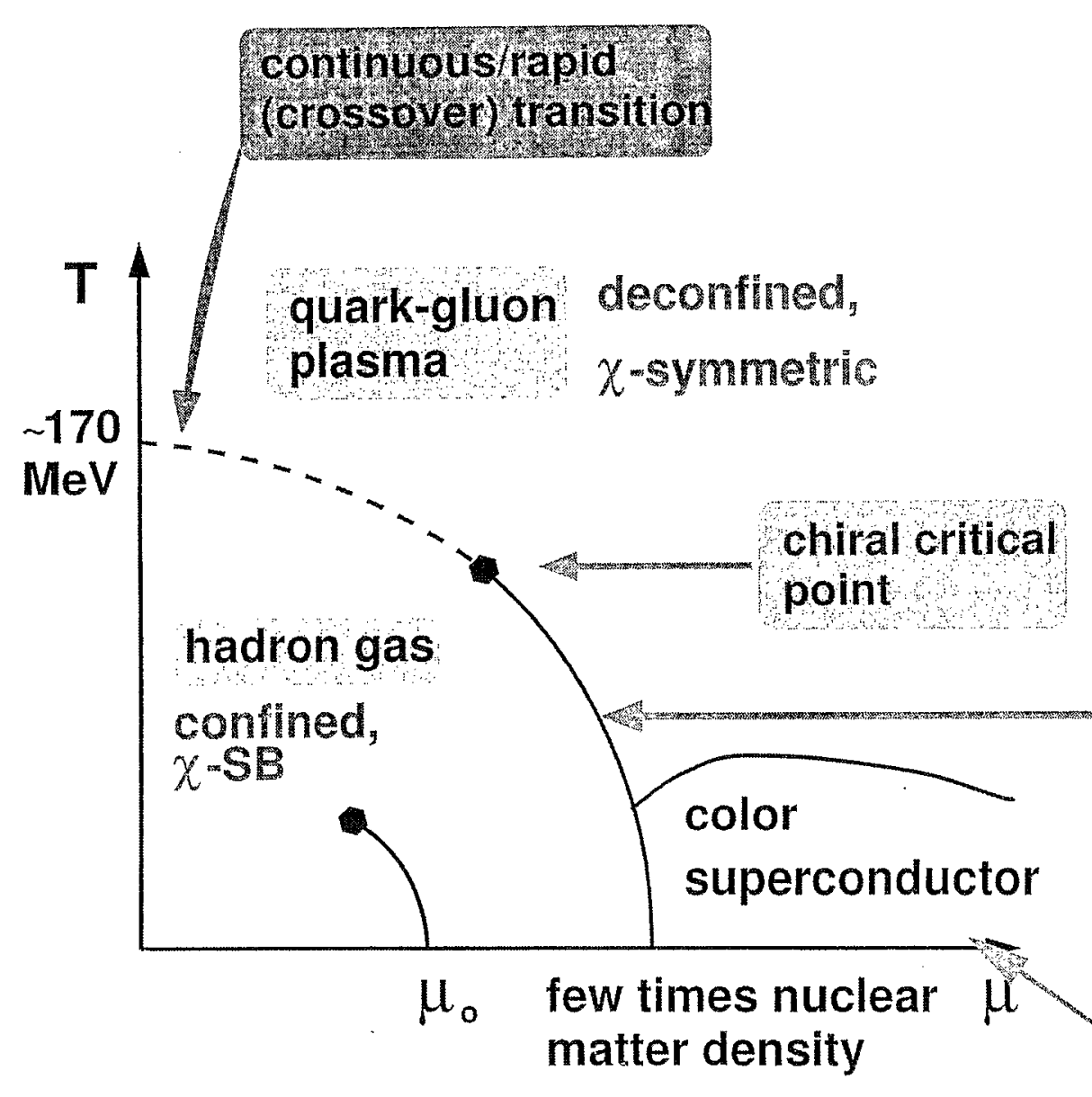

continuous transition for small chemical potential and small quark masses at

$$
\begin{aligned}
& T_{c} \simeq 170 \mathrm{MeV} \\
& \epsilon_{c} \simeq 0.7 \mathrm{GeV} / \mathrm{fm}^{3}
\end{aligned}
$$

2nd order phase transition;

Ising universality class

$T_{c}(\mu)$ under investigation

1 st order phase

transition???

expected - however, so far no direct evidence from lattice $\mathrm{QCD}$

attractive 1 -gluon exchange -> qq-condensates 


\section{Transition line for small $\mu$}

imaginary $\mu$; Taylor expansion

reweighting with exact determinant

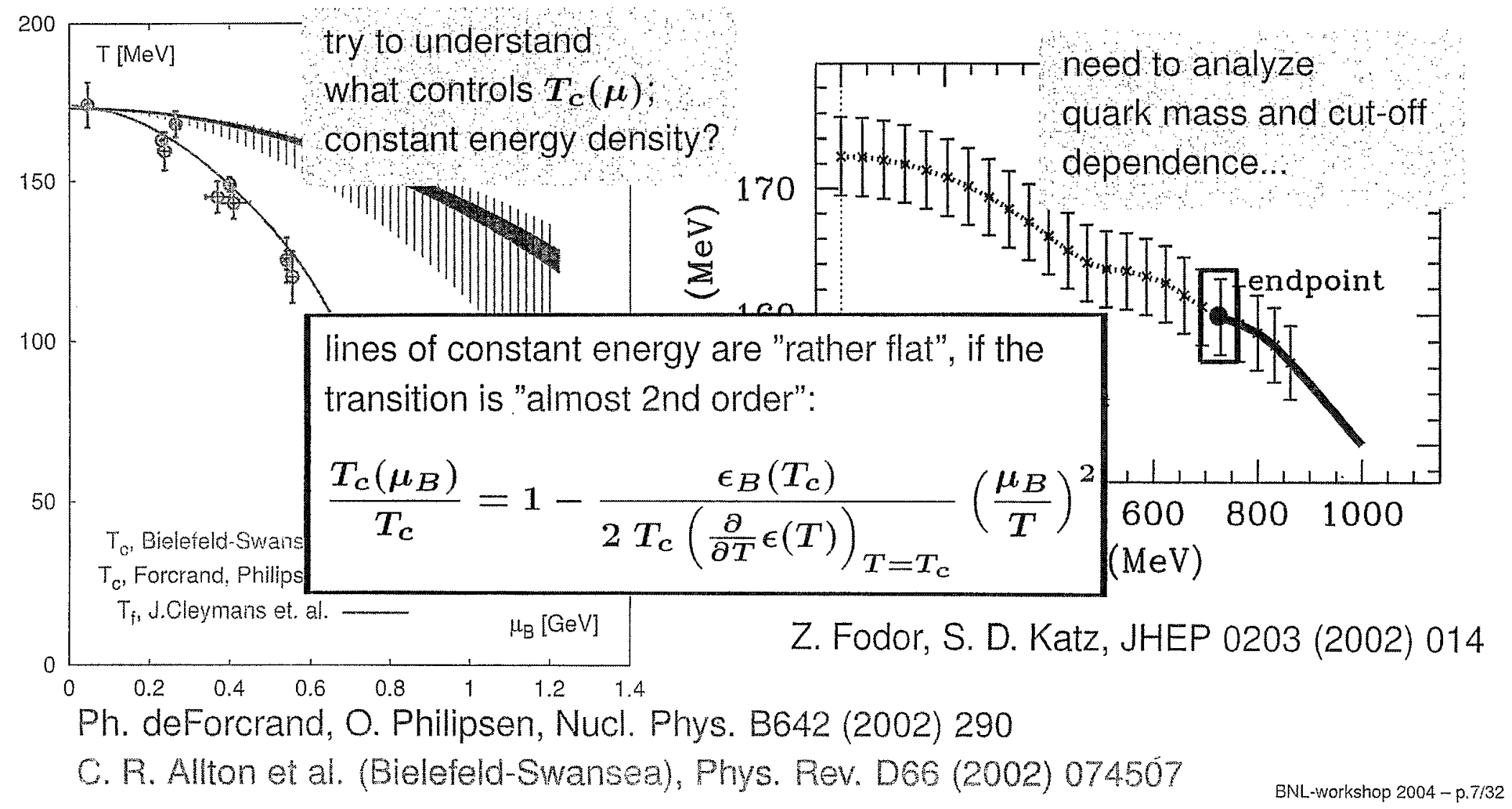




\section{Critical temperature,}

\section{equation of state}

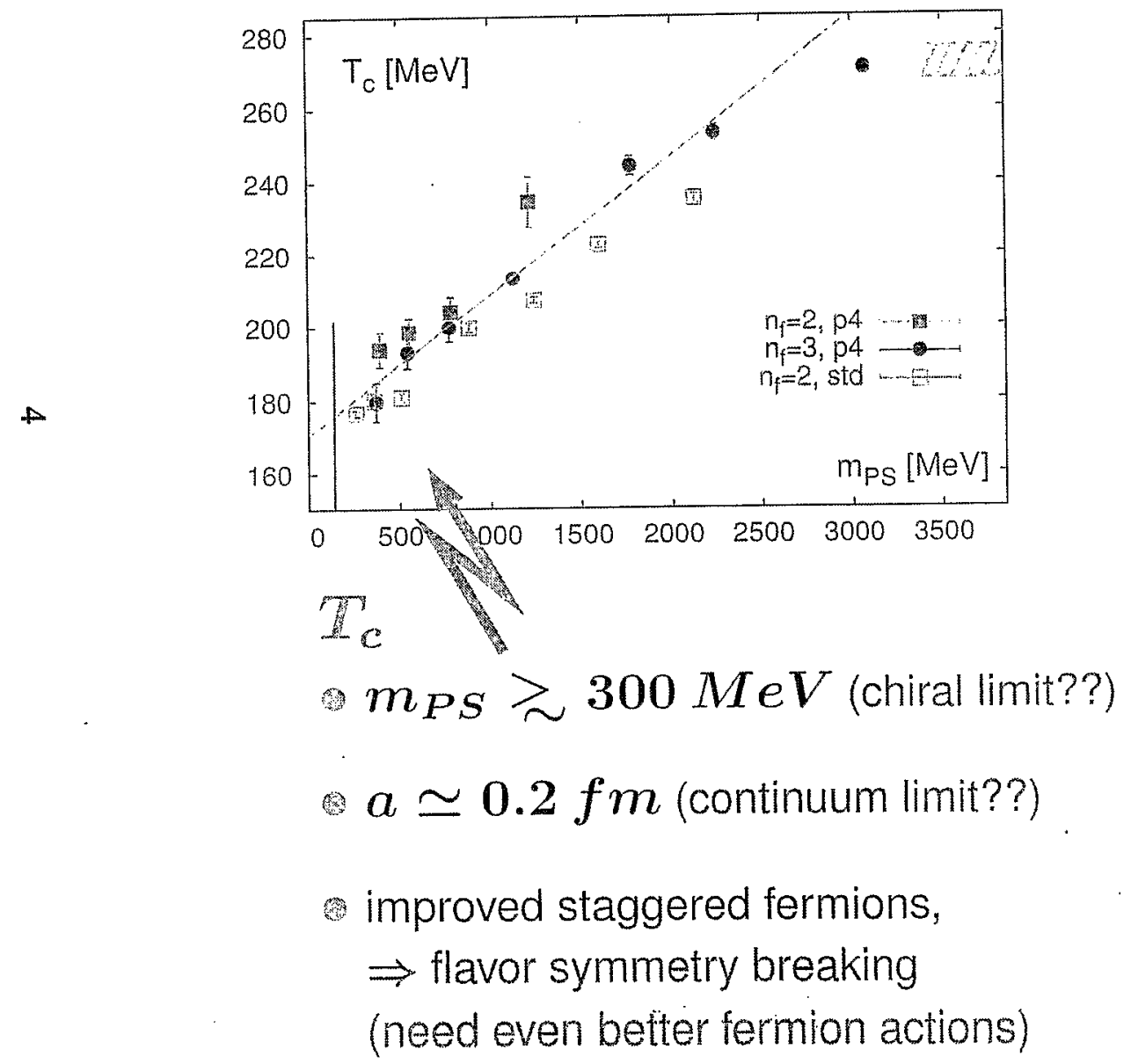

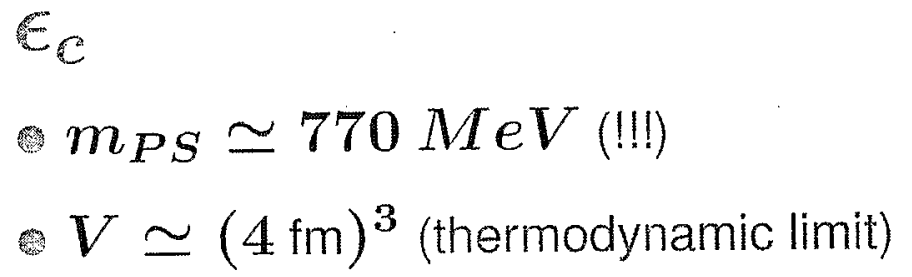
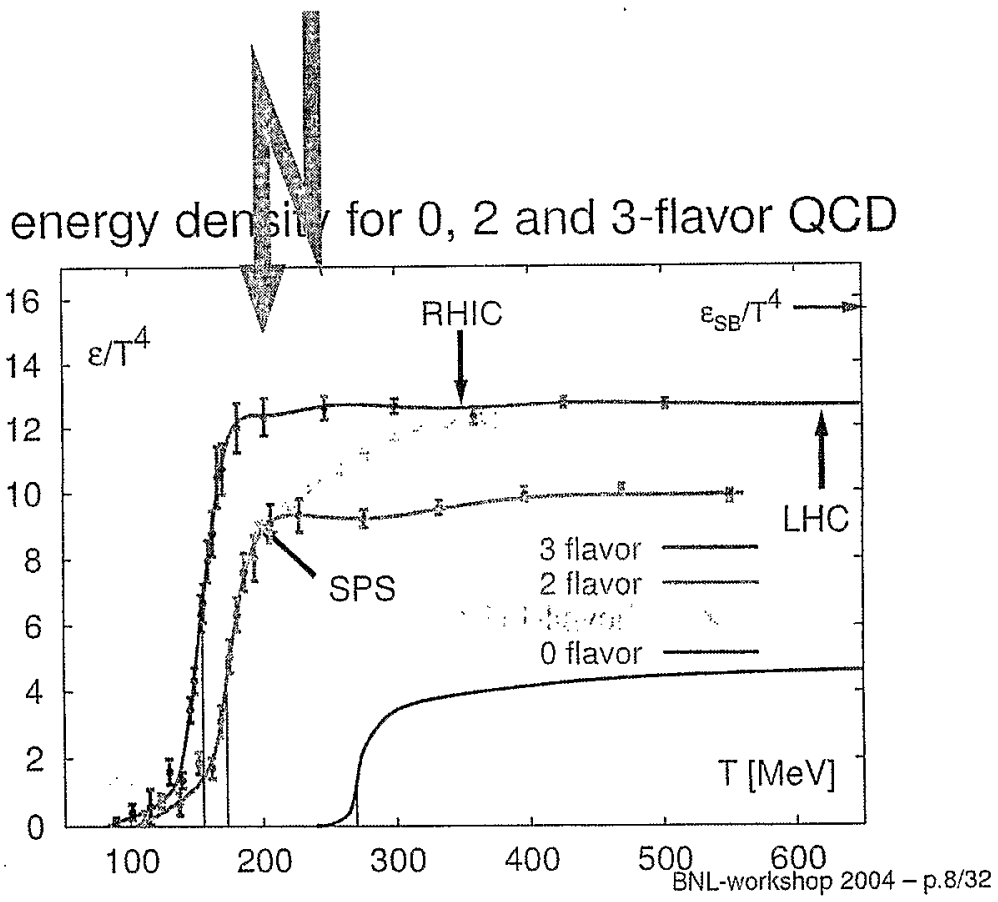


\section{Cut-off effects in the fermion sector for $\mu=0$ and $\mu>0$}

pressure on lattice with

$a T=1 / N_{\tau}=1 / 4$

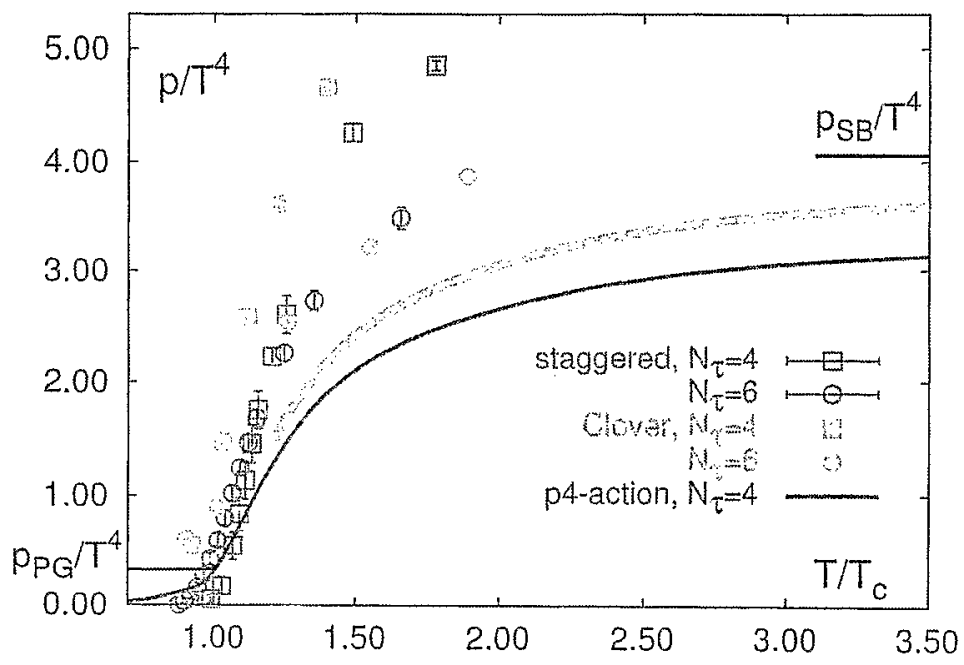

F. Karsch, E. Laermann and A. Peikert, Phys. Lett. B478 (2000) 4477

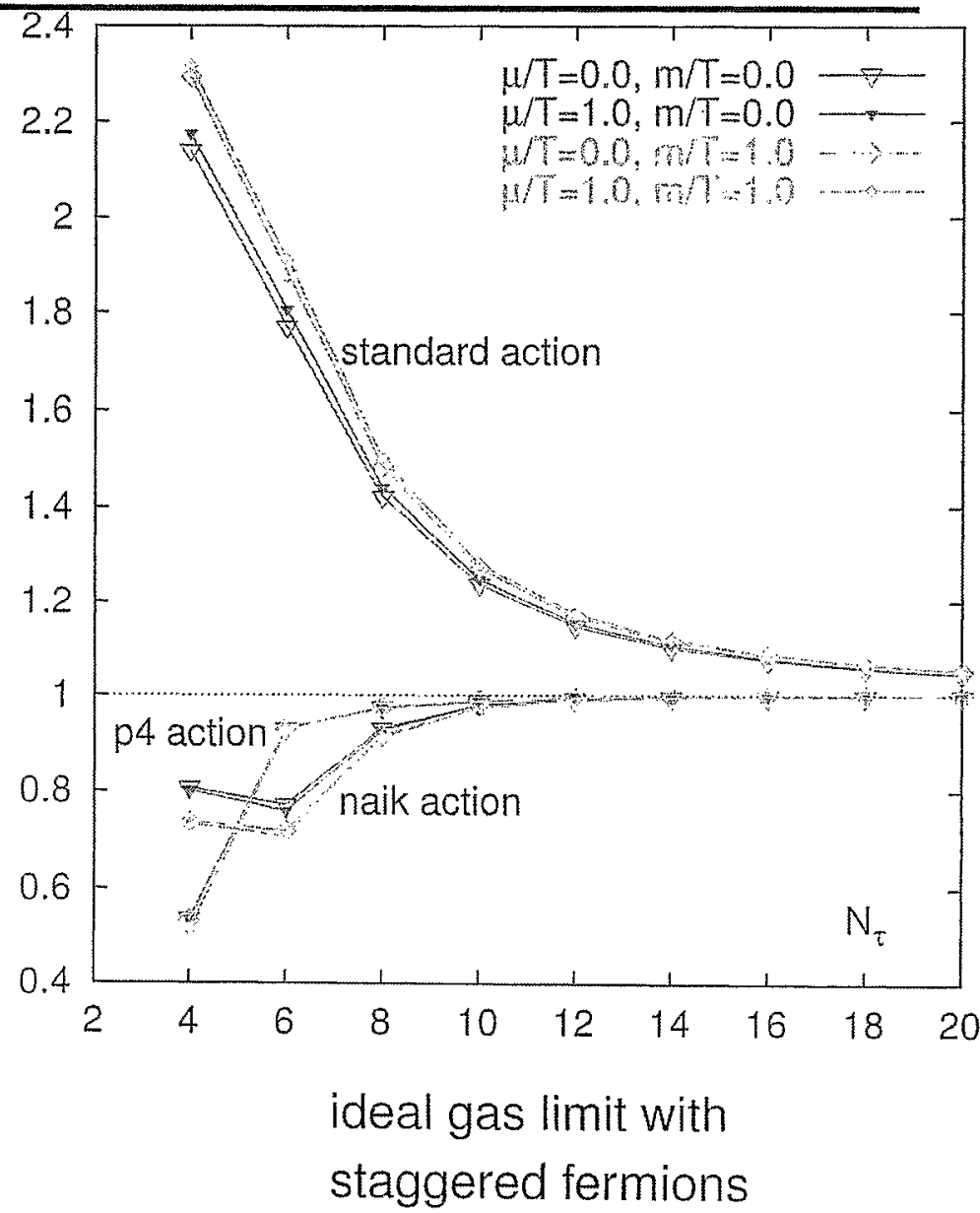

BNL-workshop $2004-p .11 / 32$ 


\section{Resonance gas: spectrum independent consequences}

FK, K. Redlich, A. Tawfik, PLB 571 (2003) 67

Boltzmann approximation $\Rightarrow$ factorization $\Rightarrow$ temperature independent ratios; spectrum independent results

$$
\frac{\Delta p}{T_{2}^{2} \chi_{q}}=\frac{1}{9}\left(1-\cosh \left(3 \mu_{q} / T\right)-\frac{\left(\frac{\mu_{q}}{T}\right)^{2}+\frac{c_{4}}{c_{2}}\left(\frac{\mu_{q}}{T}\right)^{4}+\frac{c_{6}}{c_{2}}\left(\frac{\mu_{q}}{T}\right)^{6}}{2+12 \frac{c_{4}}{c_{2}}\left(\frac{\mu_{q}}{T}\right)^{2}+30 \frac{c_{6}}{c_{2}}\left(\frac{\mu_{q}}{T}\right)^{4}}\right.
$$
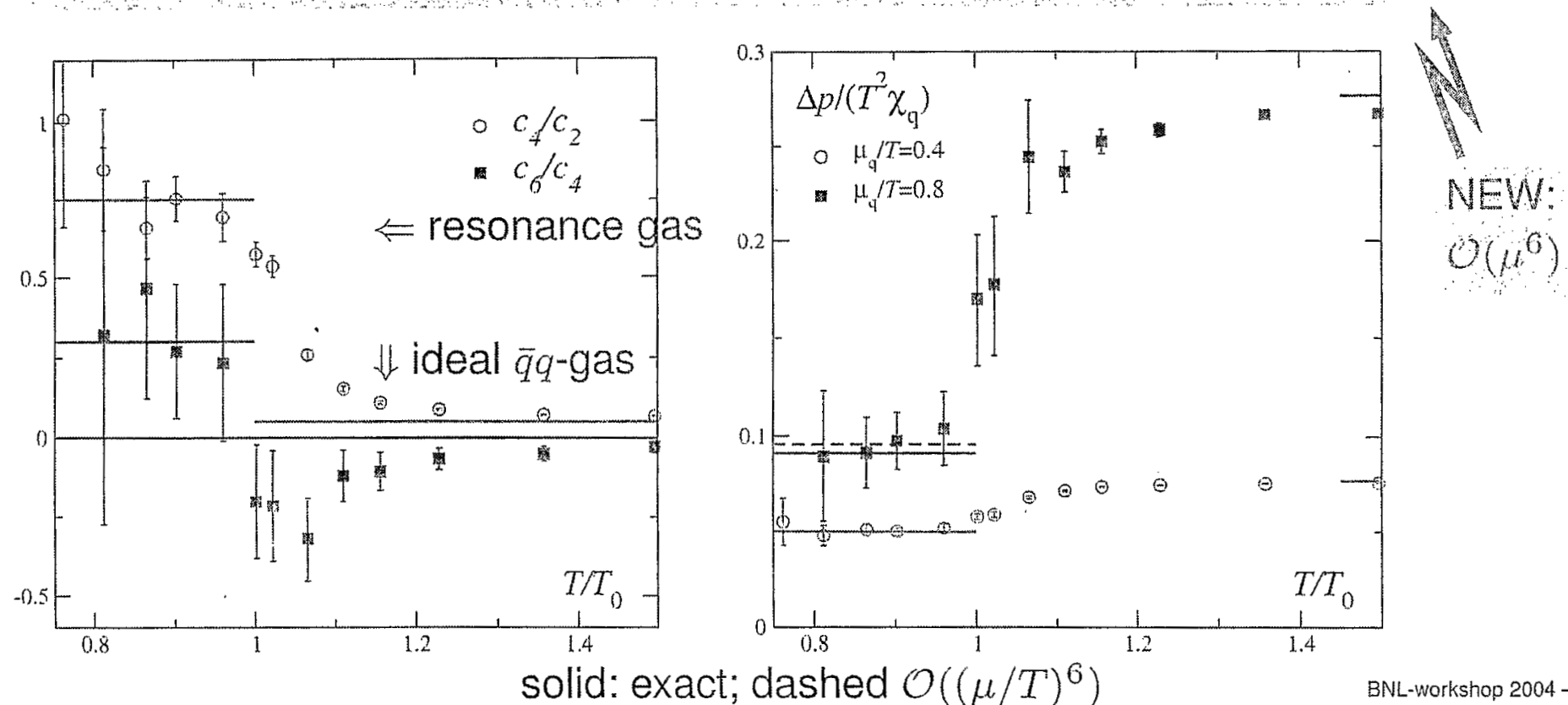


\title{
Domain Wall Fermion Thermodynamics
}

\author{
Robert D. Mawhinney \\ Columbia University
}

The first slide compares improved Wilson, improved staggered and domain wall fermions (DWF). For DWF, the chiral symmetries are present at finite lattice spacing for large $L_{s}$ (the length of the fifth dimension.)

Earlier work by the Columbia group on QCD thermodynamics for $N_{f}=2$ with DWF showed a crossover region. The second slide summarizes our simulations on $16^{3} \times 4$ and $16^{3} \times 6$ lattices. The transition looks sharper on the $N_{t}=6$ lattices. Zero temperature scale setting calculations gave $T_{c}=165 \mathrm{MeV}$ for the $N_{t}=4$ case. The $N_{t}=6$ scale setting calculation is too time consuming to do on the QCDSP computers.

Susceptibilites for Green's functions related by a $U_{A}(1)$ rotation were measured above $T_{c}$ for DWF. The results are plotted in the right-hand graph of the third slide. The expected $m^{2}$ dependence is seen. The left-hand graph shows similar results for staggered fermions, where presumably finite lattice spacing effects are distorting the expected $m^{2}$ dependence.

The fourth slide shows the residual mass for the $\beta=5.4$ DWF simulations, which have a large lattice spacing. The left-hand graph shows the residual mass at zero temperature, which exhibits a reasonable plateau for $t>5$. At finite temperature $\left(N_{t}=4\right)$ the residual mass continually changes as a function of the source-sink separation in the pseudoscalar correlator used to determine it. The residual chiral symmetry breaking resulting from finite $L_{s}$ does not appear as a constant shift in the input quark mass for these coarse lattices.

The fifth slide shows the residual mass for dynamical DWF simulations at weaker coupling with the DBW2 action done by the $\mathrm{RBC}$ Collaboration. For $\beta=0.80$, the residual mass is roughly constant, indicating that finite $L_{s}$ effects are under reasonable control. Thus with $N_{t}=10$ lattices, DWF thermodynamics studies with the full continuum symmetries are possible with known techniques. 


\section{Improved Fermion Actions}

\begin{tabular}{c|c|c|c|c} 
& $S U_{V}\left(N_{f}\right)$ & $S U_{A}\left(N_{f}\right)$ & $U_{V}(1)$ & $U_{A}(1)$ \\
\hline $\begin{array}{c}\text { Wilson } \\
\text { clover }\end{array}$ & $\sqrt{ }$ & $\begin{array}{c}\times \\
\mathcal{O}\left(a^{2}\right)\end{array}$ & $\sqrt{ }$ & $\begin{array}{c}\times \\
\mathcal{O}\left(a^{2}\right)\end{array}$ \\
\hline ASQTAD & $\times$ & $\times$ & $\sqrt{ }$ \\
staggered & $\mathcal{O}\left(a^{2}\right)$ & $\begin{array}{c}\mathcal{O}\left(a^{2}\right) \\
\text { discrete subgroup }\end{array}$ & $\begin{array}{c}U(1) \text { subgroup } \\
\left(4 N_{f} \text { flavors on lattice from fermion doubling) }\right.\end{array}$ & $\mathcal{O}\left(a^{2}\right)$ \\
\hline domain wall & $\sqrt{ }$ & $\sqrt{ }$ & $\sqrt{ }$ & $\mathcal{O}\left(a e^{-\alpha L_{s}}\right)$ \\
& \multicolumn{3}{|c}{ (for modes bound to 4-d walls) }
\end{tabular}

- Wilson: clover fermions markedly improves chiral symmetry.

- ASQTAD: improved staggered fermions have much smaller $\mathcal{O}\left(a^{2}\right)$ flavor breakin:

- DWF: decouple chiral and $a \rightarrow 0$ limits, off-shell quantities $\mathcal{O}(a)$ improved. 


\section{Finite Temperature with DWF and Wilson Gauge Action}

$$
16^{3} \times 4, m_{f}=0.02, L_{s}=24, M_{5}=1.9 \quad 16^{3} \times 6, m_{f}=0.02, L_{s}=32, M_{5}=1.8
$$

$a^{-1}=0.66 \mathrm{GeV}, T_{c}=165 \mathrm{MeV}, m_{\pi} / m_{\rho}=0.56$
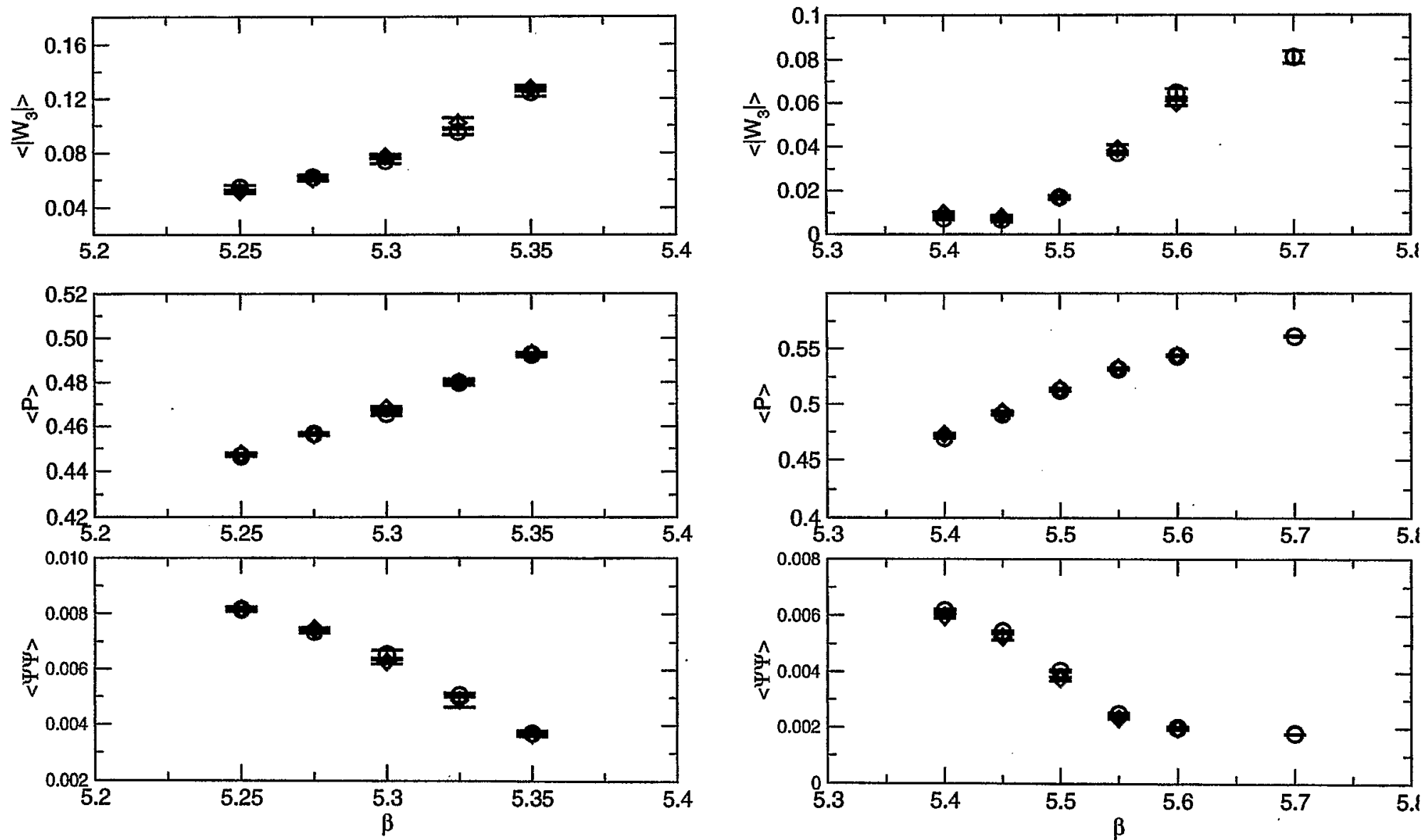


\section{Anomalous Symmetry Breaking for Staggered and DWF}

Staggered

$$
16^{3} \times 4, \beta=5.3
$$

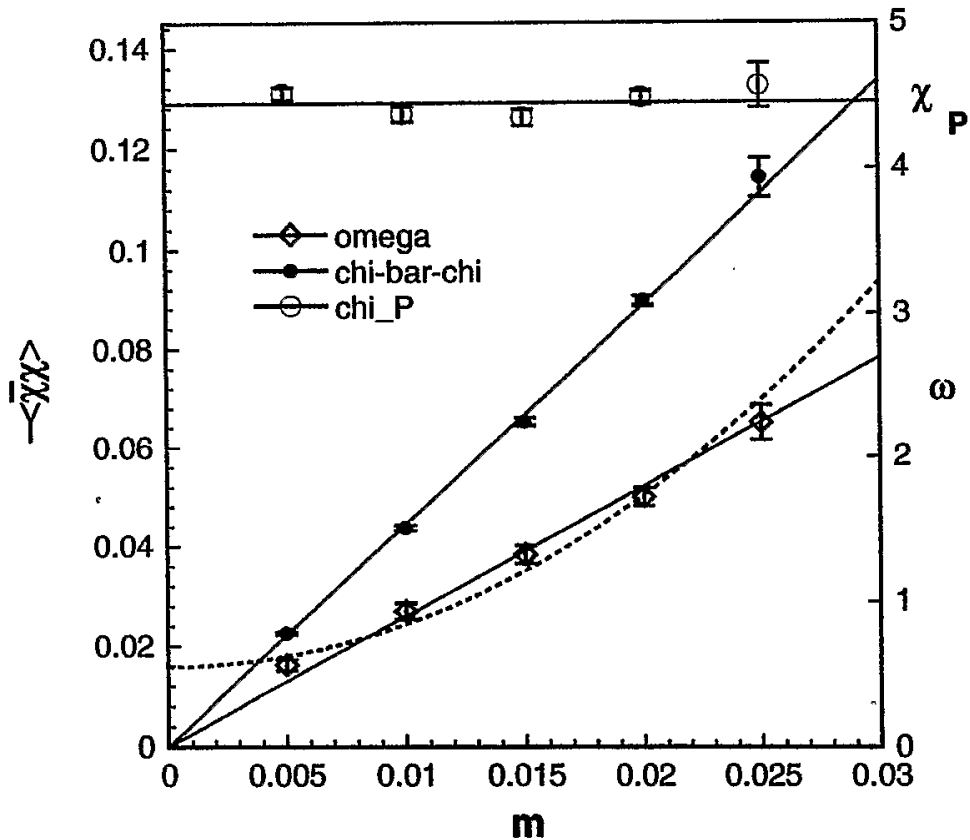

Domain Wall

$$
\begin{gathered}
16^{3} \times 4, \beta=5.40, L_{s}=16, M_{5}=1.8 \\
\chi_{\pi}=\frac{1}{2 \Omega} \int d^{4} x d^{4} y\left\langle P^{j}(x) P^{j}(y)\right\rangle \\
P^{j}(x) \equiv i \bar{\psi} \tau^{j} \gamma^{5} \psi(x)
\end{gathered}
$$$$
\omega \equiv \chi_{\pi}-\chi_{S}
$$

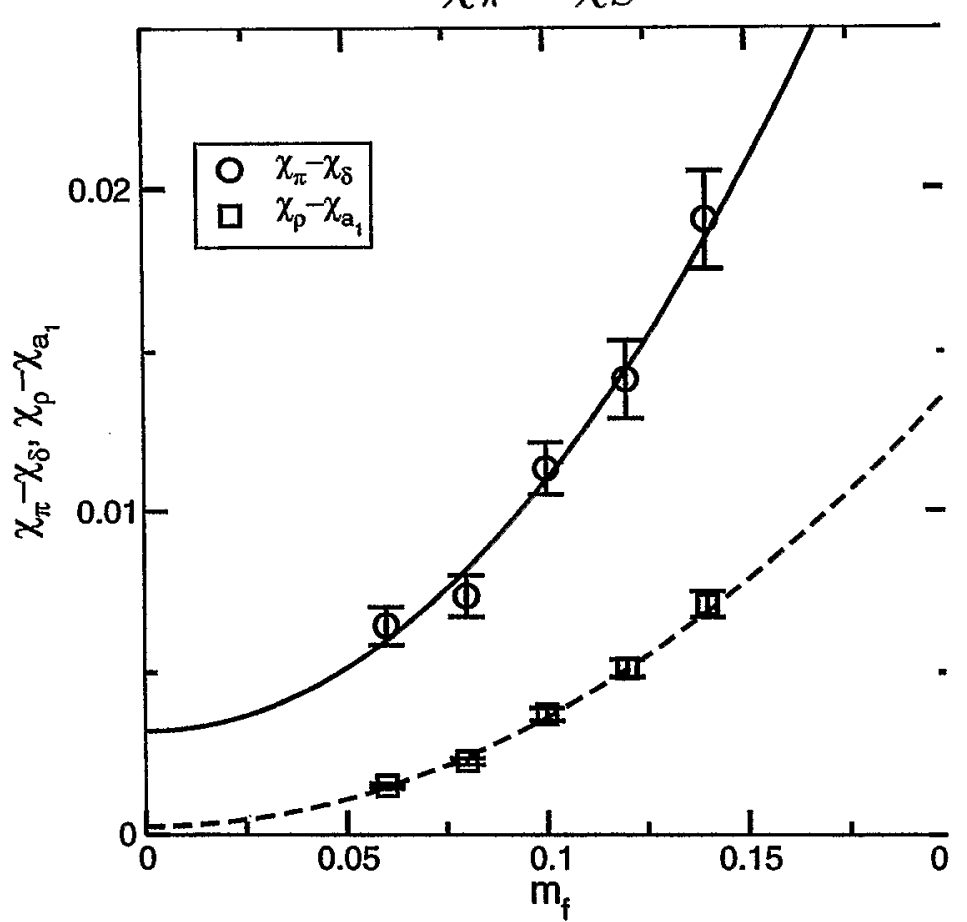




\section{Residual mass at finite $\mathbf{T}$}

Zero temperature, $8^{3} \times 32$

$$
\begin{aligned}
& \beta=5.40, m_{f}=0.06 \\
& L_{s}=16, M_{5}=1.90
\end{aligned}
$$

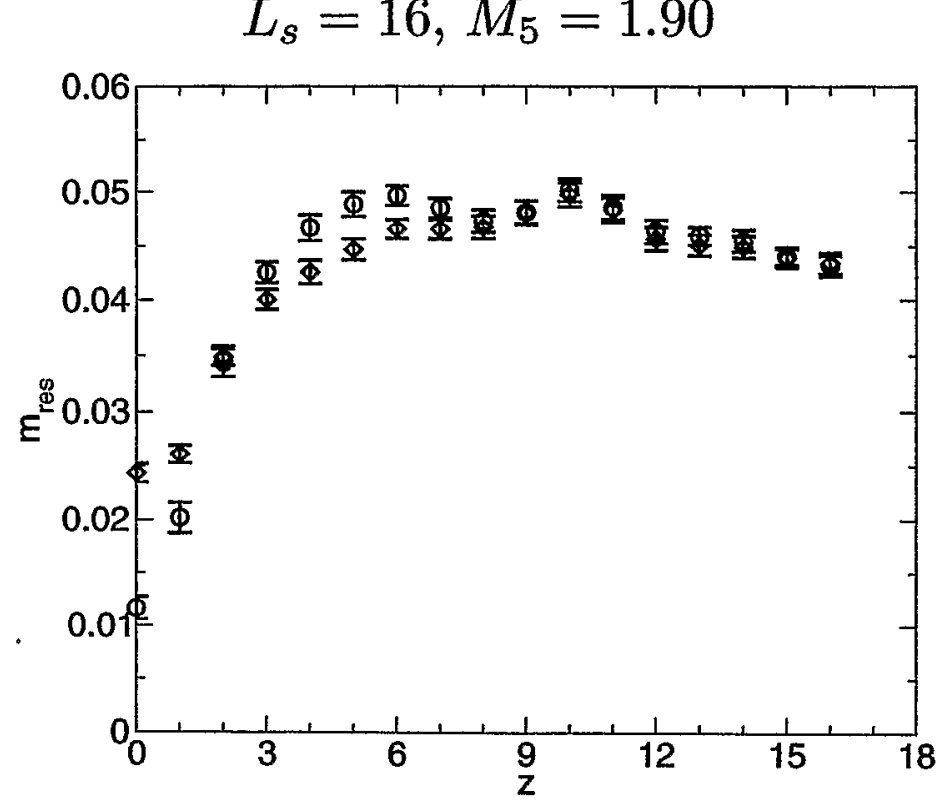

Above $T_{c}, 16^{3} \times 4$

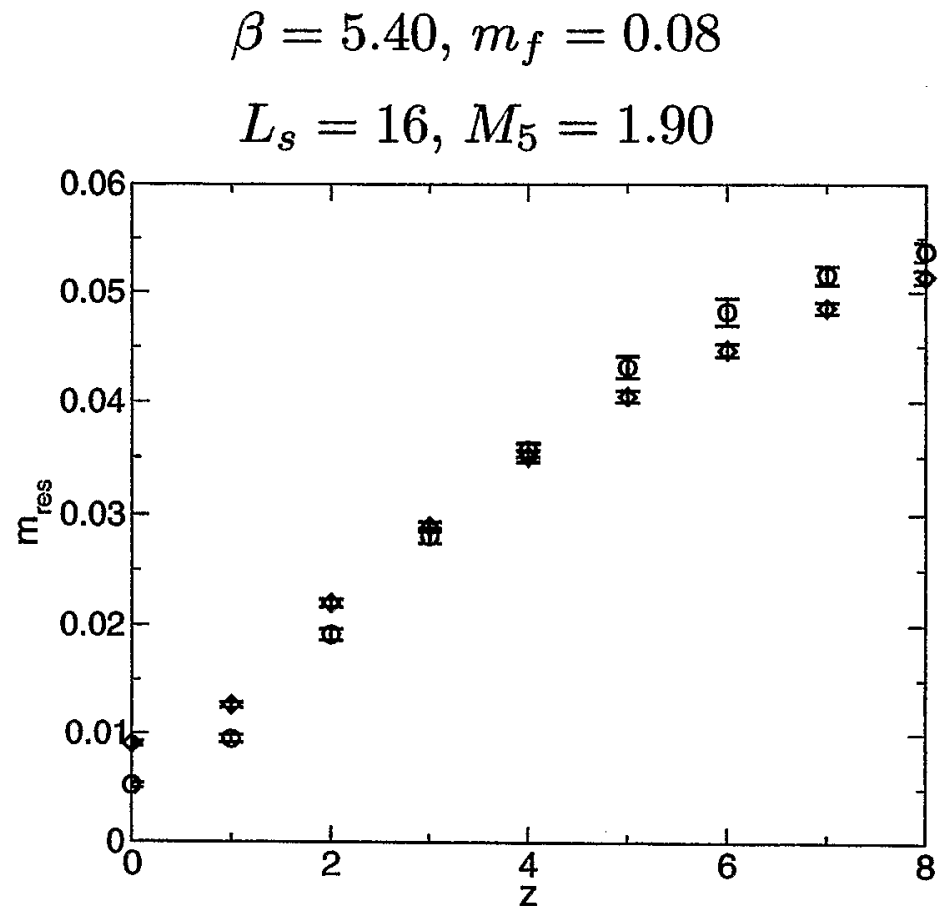

Residual mass is not well defined at finite $T$ on these coarse lattices 


\section{Dynamical DBW2 $m_{\mathrm{res}}$ for $T>T_{c}$}

\begin{tabular}{llll}
\hline \hline$\beta$ & $m_{f}$ & $N_{t}$ & $m_{\rho}$ \\
\hline \hline 0.75 & 0.022 & 4 & $1.465(3)$ \\
0.80 & 0.020 & 4 & $1.472(3)$ \\
0.80 & 0.020 & 8 & $0.737(6)$ \\
\hline \hline
\end{tabular}

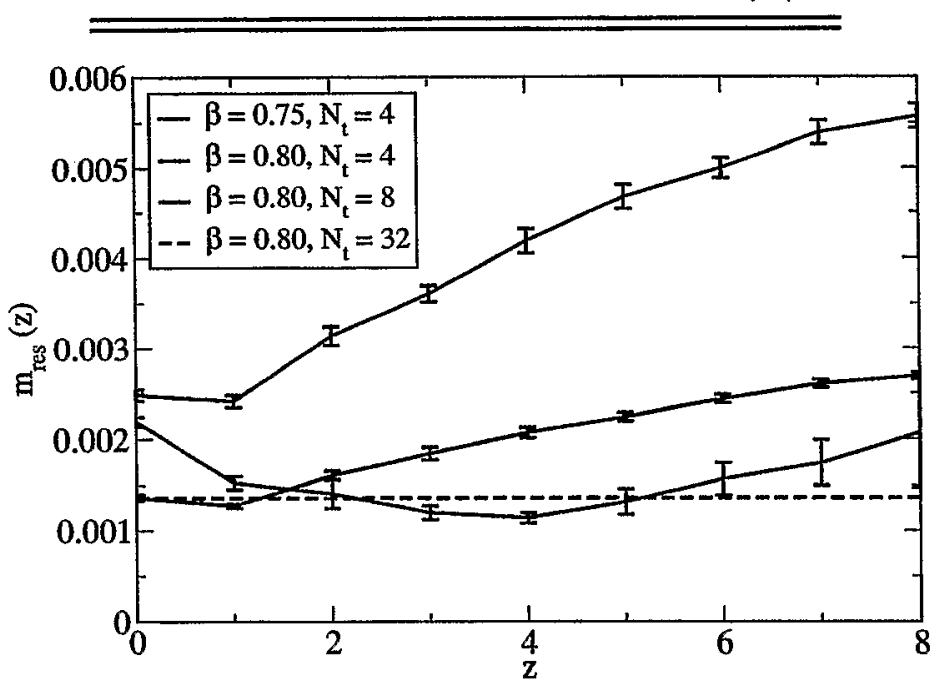

Results from $16^{3}$ spatial volumes with $M_{5}=1.8$ 
Urs M. Heller

\title{
Phase diagram of finite temperature QCD with improved staggered fermions
}

\author{
Urs M. Heller \\ APS, Ridge \\ \& $B N L$
}

\begin{abstract}
With the MILC collaboration:
C. Aubin, C. Bernard, T. Burch, C. DeTar, Steven Gottlieb, E. Gregory, UMH, J. Hetrick,

J. Osborn, R. Sugar, and D. Toussaint.
\end{abstract}

- Introduction and Motivation

- The Quark Action and its Properties

- Simulation Strategy

- Numerical Results

- Quark Number Susceptibilities

- Conclusions

BNL workshop "Lattice QCD at finite temperature and density"

February 8, 2004. 


\section{Introduction}

With the Relativistic Heavy Ion Collider (RHIC) now producing data, it has become even more important to understand the phase diagram of QCD at finite temperature, and to determine properties of the high temperature quark-gluon-plasma phase with confidence, i.e. with controlled lattice spacing errors.

For this, we employed the improved staggered "asqtad" quark action, with a "fat link" to improve flavor symmetry (see left plot) and a Naik (3-hop) term to improve the dispersion relation, which decreases lattice artifacts in the high temperature phase (see right plot).
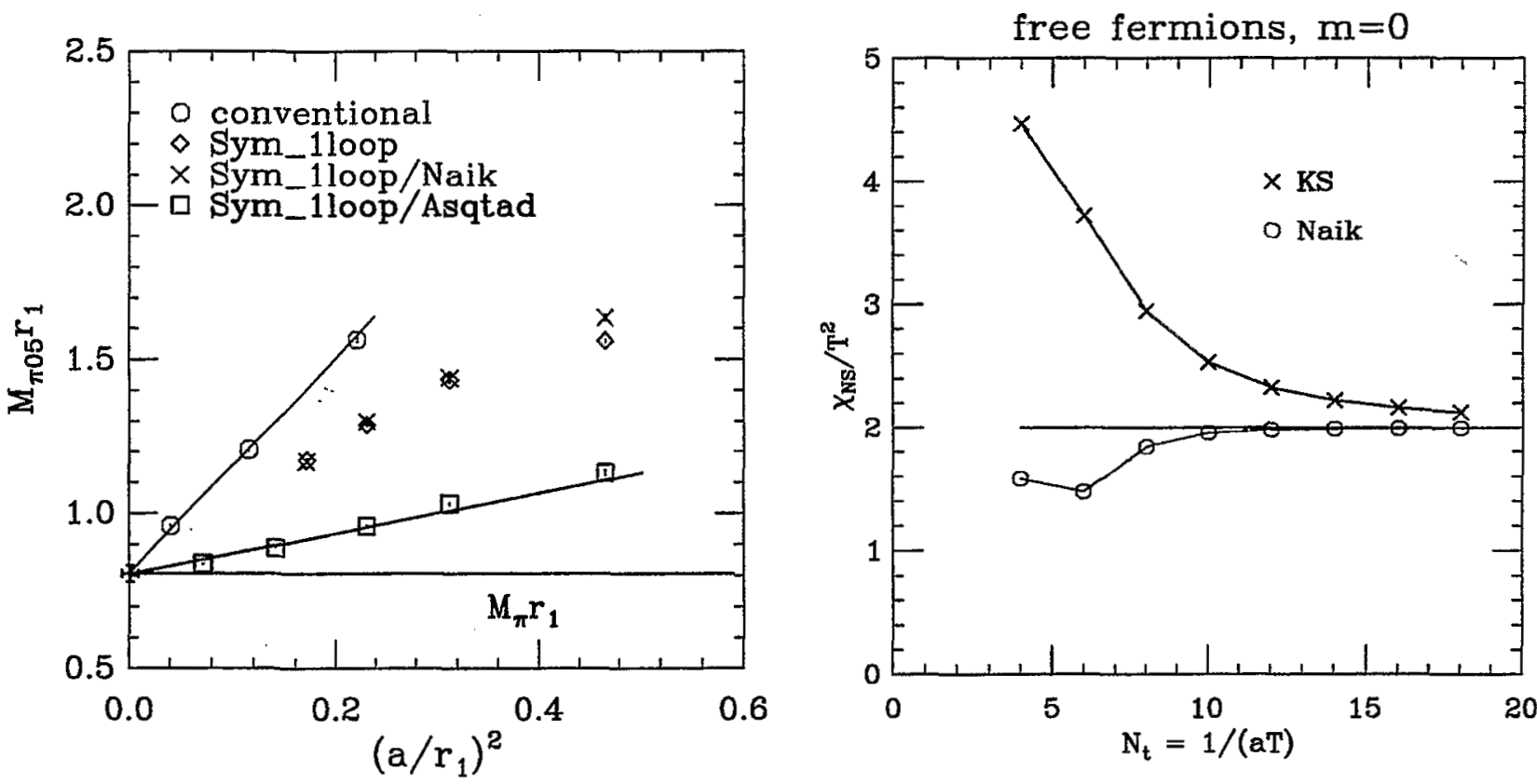

For zero temperature results see also PRL 92 (2004) 022001. 
Urs M. Heller

\section{Numerical Results}

We made simulations for $N f=3$ degenerate flavors, and for $N f=2+1$ flavors with one quark fixed at the strange quark mass, doing simulations along "curves of constant physics". $\langle R e P\rangle$ is an indicator of (de-) confinement:
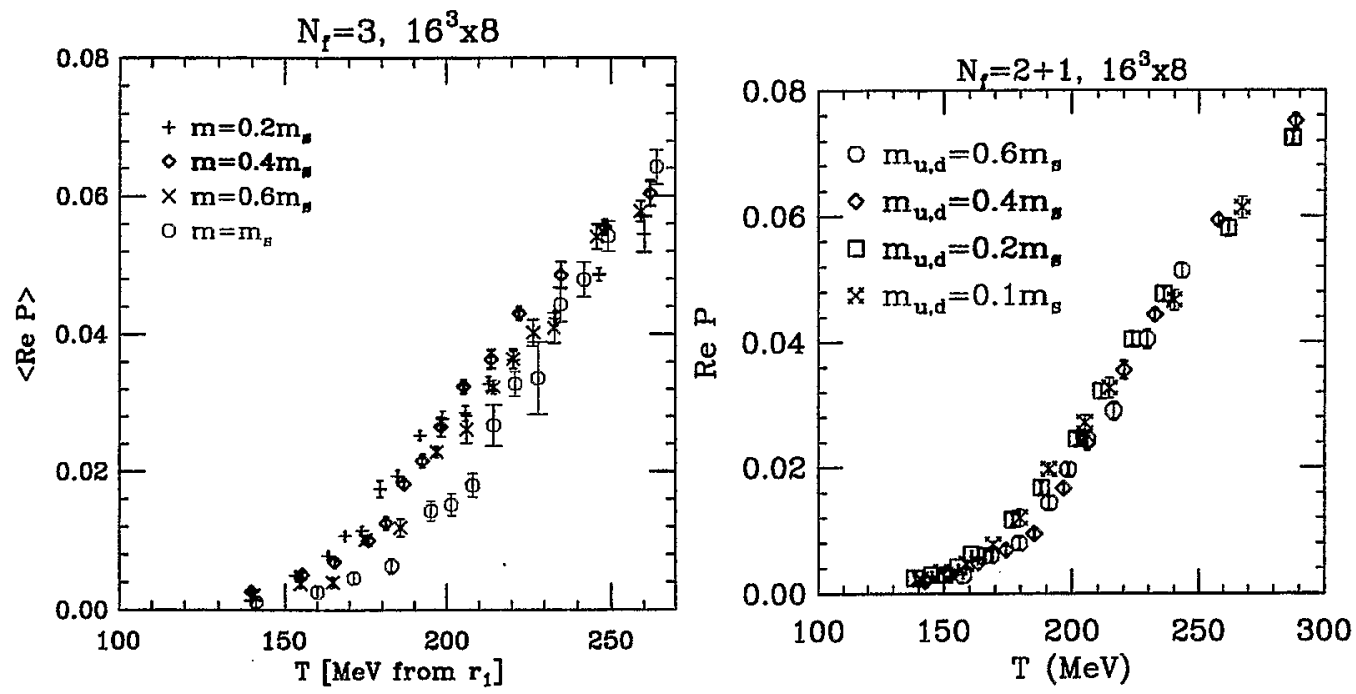

$\langle\bar{\psi} \psi(T)\rangle$ signals chiral symmetry breaking and restoration. It is shown with an extrapolation to the chiral limit.
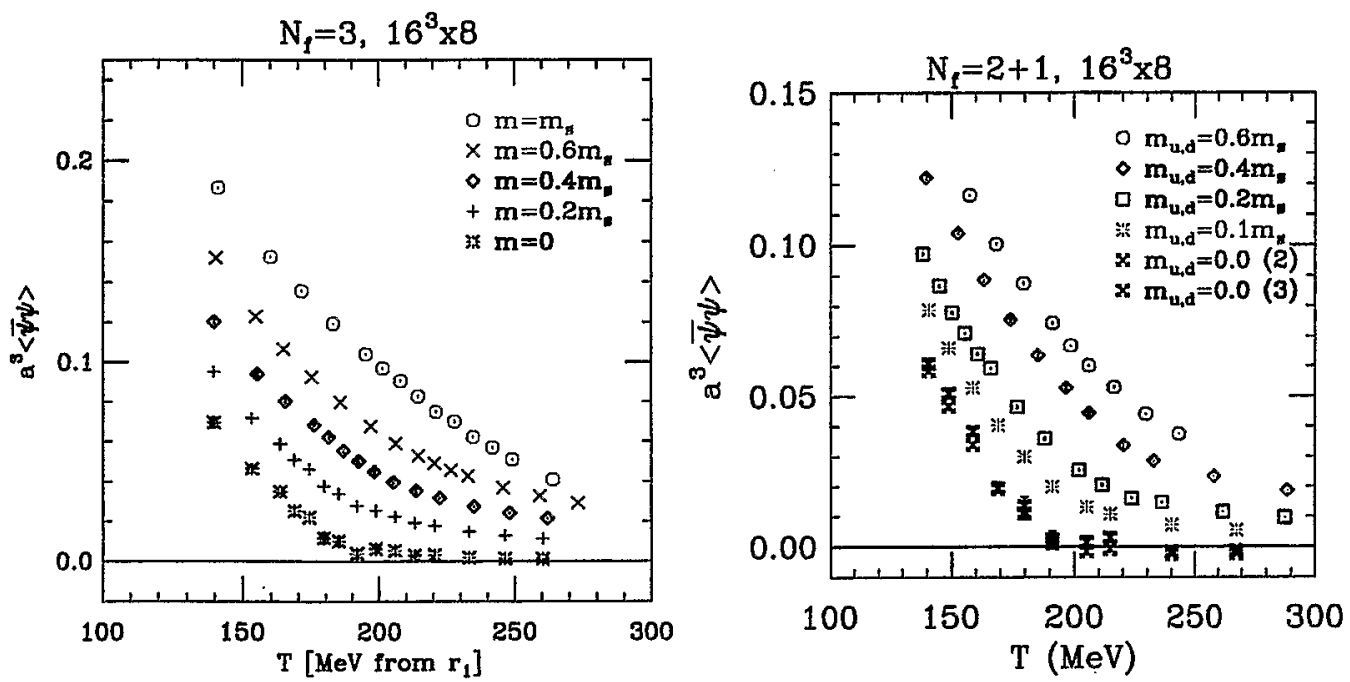
Urs M. Heller

To locate the crossover/transition we measured the " $\bar{\psi} \psi$-susceptibilities"
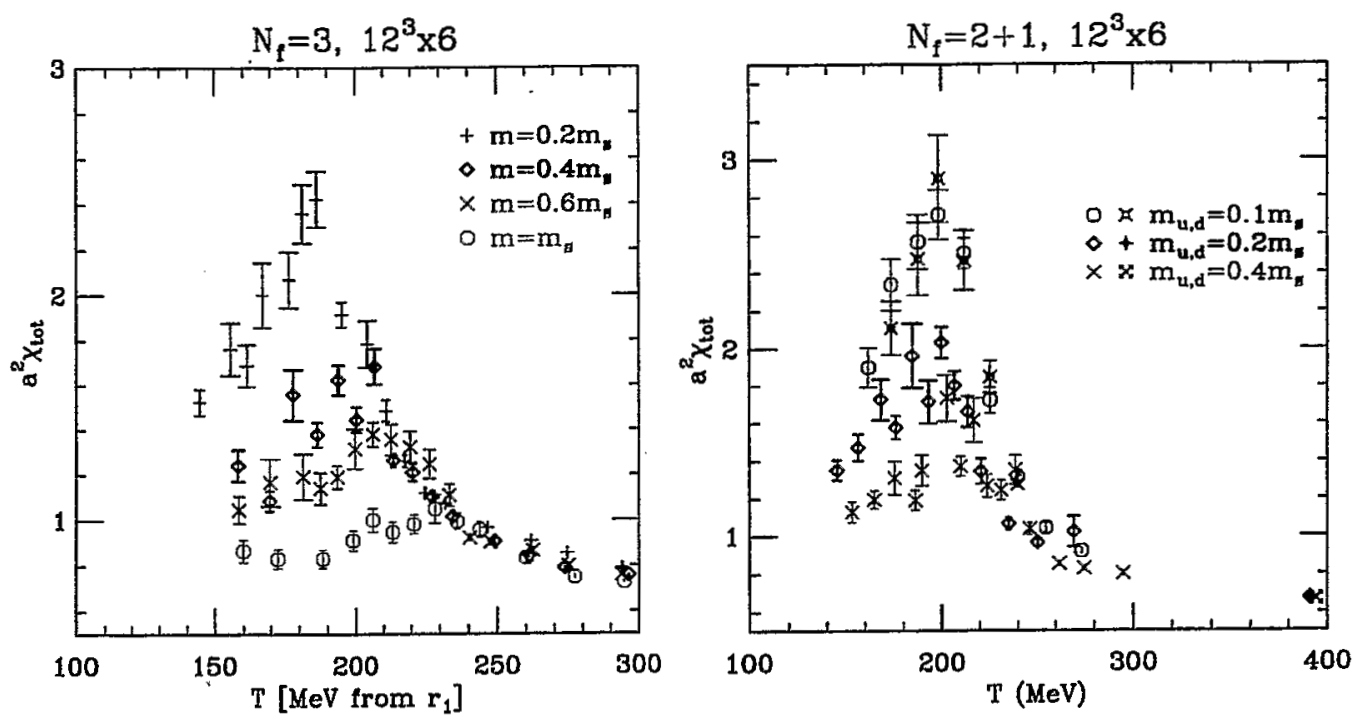

From the peak positions we inferred the values of $r_{1} T_{c}$ :
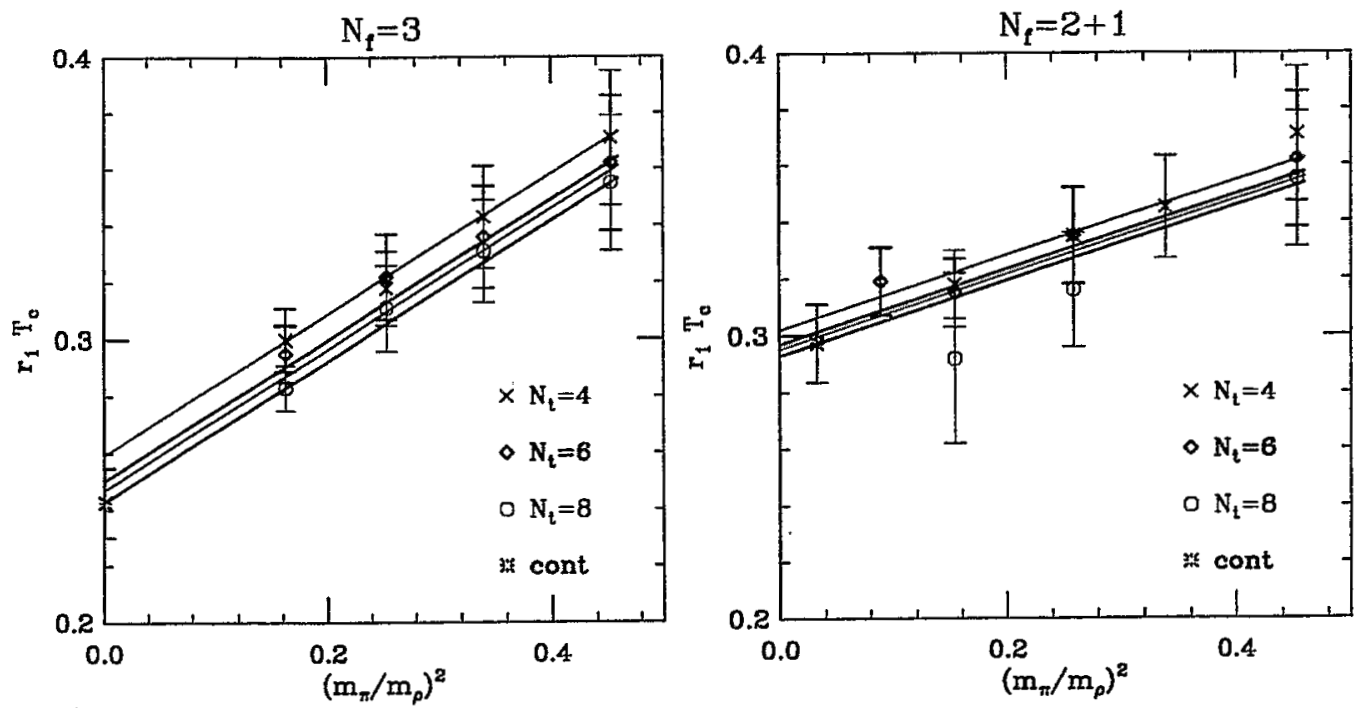

Giving $T_{c}=151(7)(3) \mathrm{MeV}$ for $N_{f}=3$ and $T_{c}=185(9)(4) \mathrm{MeV}$ for $N_{f}=2+1$. 
Urs M. Heller

\section{Quark number susceptibilities}

We also measured quark number susceptibilities, which have small lattice artifacts with the improved quark action:
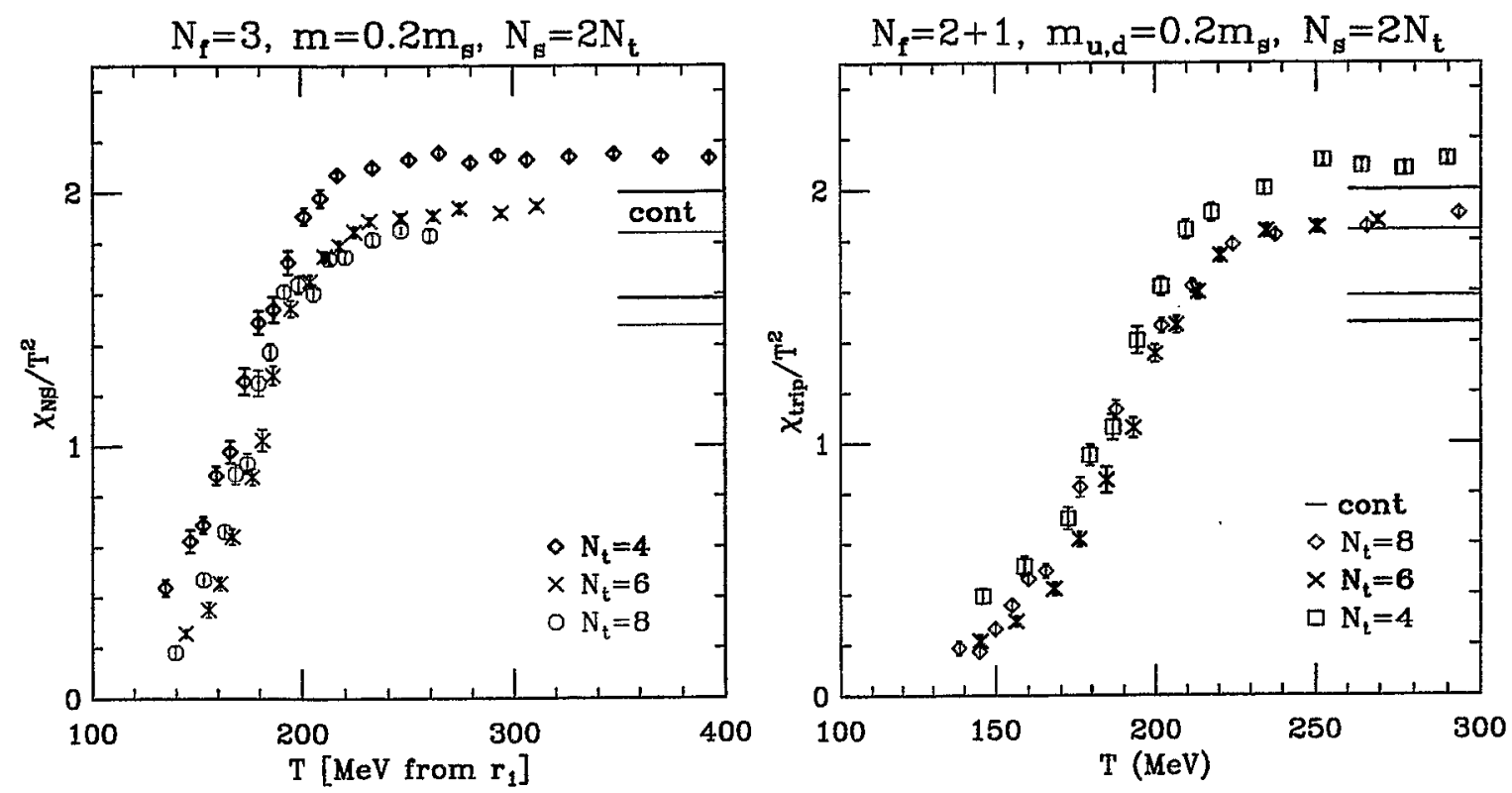

The difference between singlet and triplet susceptibility is visible around the transiton/crossover
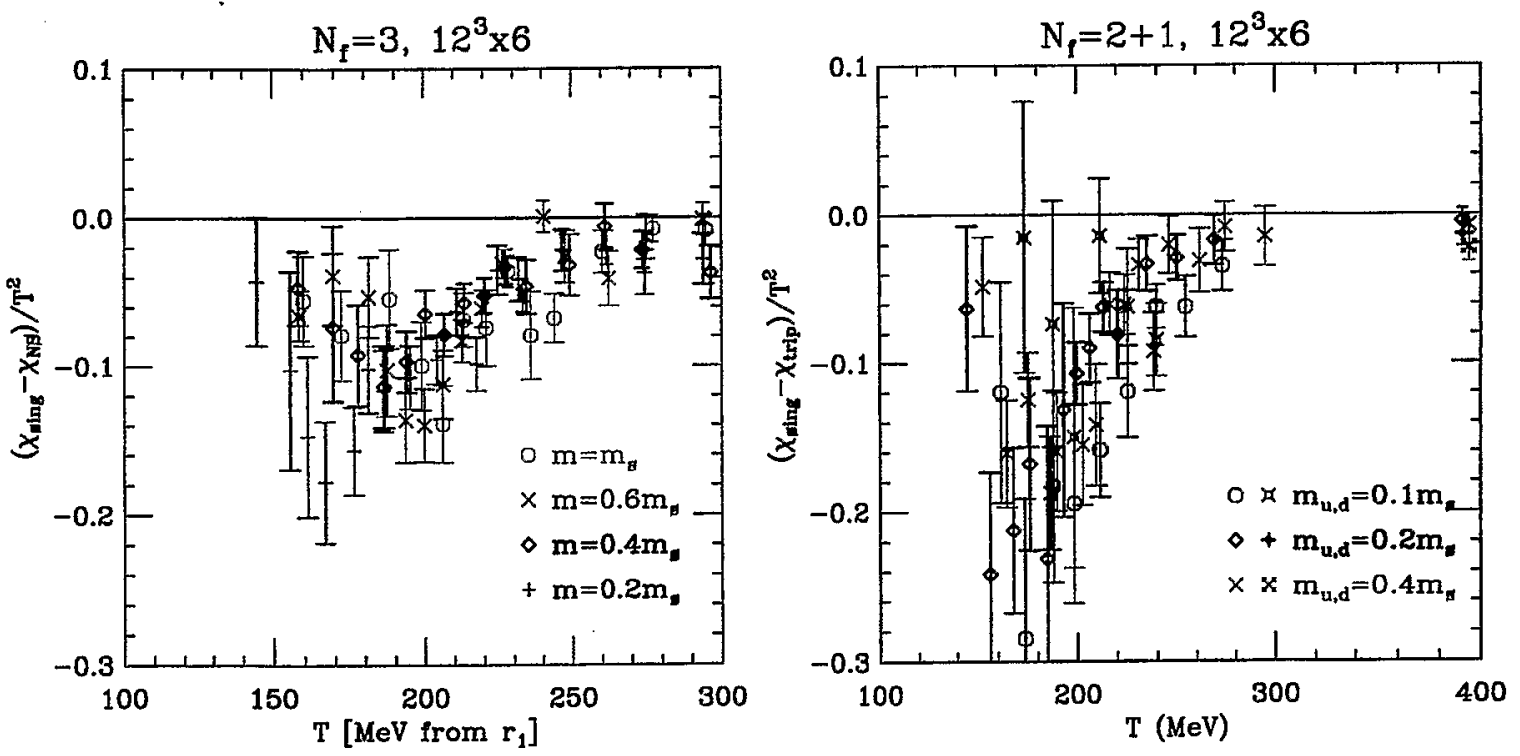

APS, Ridge \& BNL

February 2004 
We extended these calculations to higher temperature:
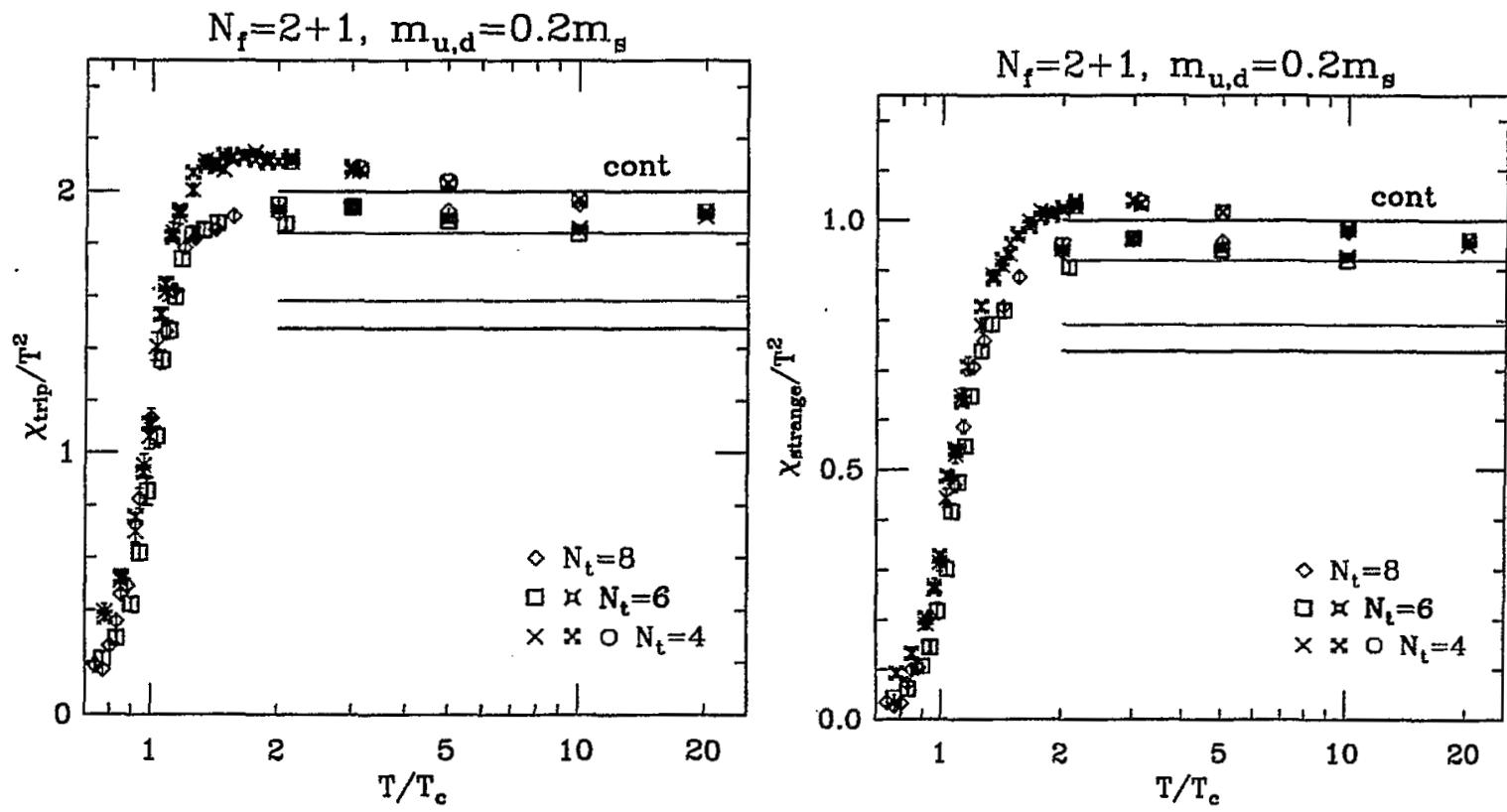

We see an indication that lattice artifacts are different around $T_{c}$, where the results from $N_{t}=6$ and 8 agree, within errors, and at larger temperatures, where the data start going towards their extreme high tempertaure, i.e. free field, values.

\section{Conclusions}

An improved staggered fermion action, the asqtad action, with reduced flavor symmetry breaking and better dispersion relation, appears to give improved results, with smaller lattice artifacts, not only in zero temperature simulations but also at finite temperature. Thus simulations at small $N_{t}$ are already close to the continuum limit. 
Lattice QCD at finite $T$ and $\mu$, phase diagram and the critical point

Zoltán Fodor

Z. Fodor, S. D. Katz, hep-lat/0104001 (PLB 534 (2002) 87)

Z. Fodor, S. D. Katz, hep-lat/0106002 (JHEP 03 (2002) 014)

Z. Fodor, S. D. Katz, hep-lat/0402006

1. Introduction

2. Overlap improving multi-parameter reweighting

3. Phase diagram, critical endpoint in $n_{f}=2+1$ dynamical QCD

4. (Equation of state at finite $\mu$ : S.D. Katz)

5. Summary 


\section{QLU witn $n_{f}=\angle+\perp$ aynamical staggerea Termions}

Z. Fodor, S. D. Katz, hep-lat/0106002 (JHEP 03 (2002) 014)

- partition function with multi-parameter reweighting

$$
\begin{gathered}
Z(\alpha)=\int \mathcal{D} \phi \exp \left[-S_{b o s}\left(\alpha_{0}, \phi\right)\right]\left[\operatorname{det} M\left(\phi, \alpha_{0}\right)\right]^{n_{f} / 4} \\
\left\{\exp \left[-S_{b o s}(\alpha, \phi)+S_{b o s}\left(\alpha_{0}, \phi\right)\right]\left[\operatorname{det} M(\phi, \alpha) / \operatorname{det} M\left(\phi, \alpha_{0}\right)\right]^{n_{f} / 4}\right\}
\end{gathered}
$$

we measure fractional powers of the complex determinants $\Rightarrow$ choose among the possible Riemann-sheets

a. gauge fix to $A_{0}=0$ on all but the last timeslice

b. multiply the $j$-th row/column by $e^{ \pm j \mu}$

c. rearrange the columns of the matrix

d. $L_{t}-2$ Gauss elimination step gives a $6 L_{s}^{3} \times 6 L_{s}^{3}$ matrix

$$
\operatorname{det} M(\mu)=e^{-3 V \mu} \prod_{i=1}^{6 L_{s}^{3}}\left(e^{L_{t} \mu}-\lambda_{i}\right)
$$

$\Rightarrow$ gives $\mathbf{Z}$ for "arbitrary" $\mu$ and $\beta$ 
Z. Fodor, S. D. Katz, hep-lat/0402006 (140 times more CPU demanding)

- three basic steps of the analysis $m_{s}=0.25, m_{u d}=0.0092$ : physical ones, $\mathrm{T}=0$ measurements show three times smaller light quark masses as in the previous case

a. determine the transition points, $\operatorname{Re}\left(\beta_{0}\right)$, on $L_{s}=6,8,10,12$ three times larger physical volumes as in the previous case $\beta_{c}$ as a function of $\mu$ by the Lee-Yang zeros for $\mu \neq 0$ overlap improving multi-parameter reweighting $100 \mathrm{k}, 100 \mathrm{k}, 100 \mathrm{k}, 150 \mathrm{k}$ configurations, respectively every 50th configuration treated as independent (few thousend)

b. by inspecting the $V \rightarrow \infty$ limit of $\operatorname{Im}\left(\beta_{0}\right)$ separate the crossover and the $1^{\text {st }}$ order $\mathrm{PT}$ regions in $\mu$

c. connect $\mu=\mathrm{T}=0$ lattice parameters with observables: physical scale by $R_{0}(1 / 403 \mathrm{MeV})$ and $m_{\rho}(770 \mathrm{MeV})$ $\left(3 \times 3000\right.$ configurations on $12^{3} \cdot 24$ lattices $)$ 
- separate the crossover and the $1^{\text {st }}$ order PT $V \rightarrow \infty$ limit of $\operatorname{Im}\left(\beta_{0}\right)$ as a function of $\mu$

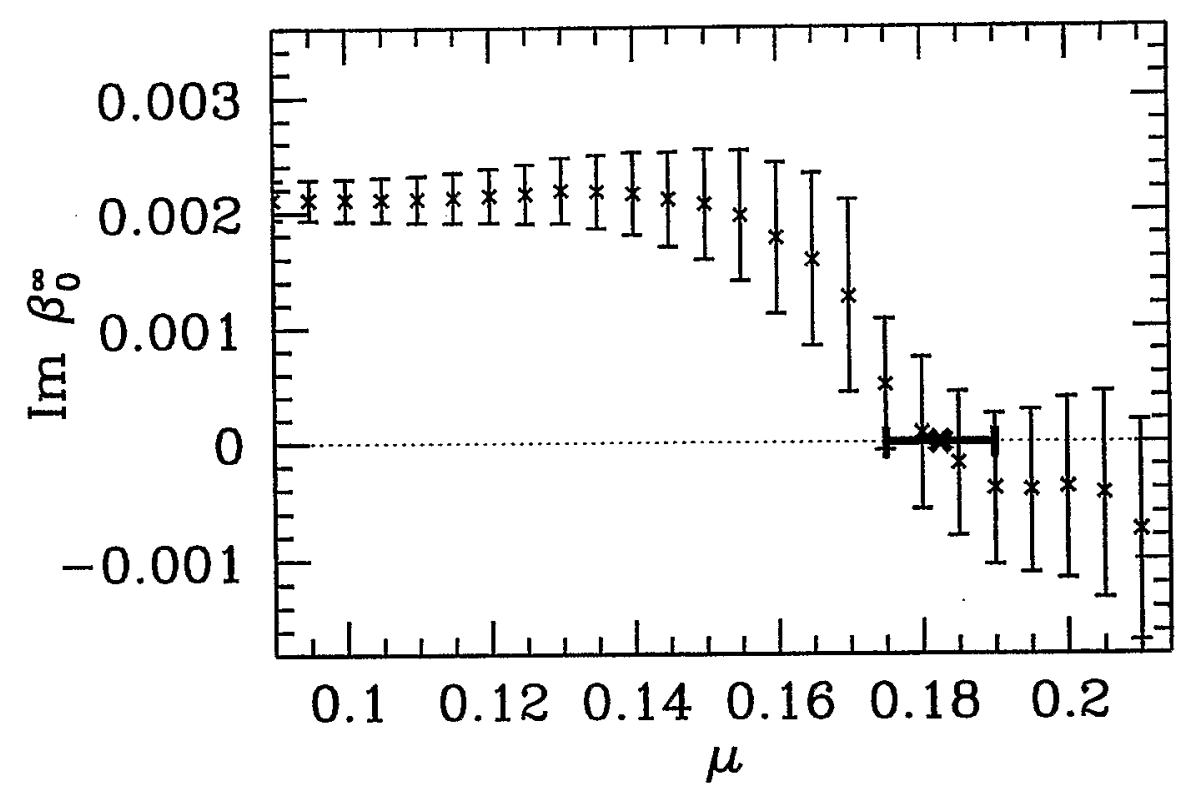

small $\mu$ : $\operatorname{Im}\left(\beta_{0}^{\infty}\right)$ inconsistent with $0 \Rightarrow$ crossover increasing $\mu$ : $\operatorname{Im}\left(\beta_{0}^{\infty}\right)$ decreases $\Rightarrow$ transition becomes consistent with a $1^{\text {st }}$ order $\mathrm{PT}$

endpoint chemical potential: $\mu_{e n d}=0.183(8)$ 
- overall scale and $\beta$-function

$\tilde{\omega}$

\begin{tabular}{l|l|l|l|l|l}
$\operatorname{Re}(\mu)$ & 0.04 & 0.08 & 0.12 & 0.16 & 20 \\
\hline $\operatorname{Re}\left(\beta_{0}\right) ; L_{s}=6$ & $5.1863(9)$ & $5.1839(9)$ & $5.1800(9)$ & $5.1749(11)$ & $5.1713(14)$ \\
$10^{2} \operatorname{Im}\left(\beta_{0}\right)$ & $2.39(6)$ & $2.39(6)$ & $2.41(8)$ & $2.41(13)$ & $2.26(22)$ \\
\hline $\operatorname{Re}\left(\beta_{0}\right) ; L_{s}=8$ & $5.1886(4)$ & $5.1858(5)$ & $5.1811(5)$ & $5.1753(7)$ & $5.1710(16)$ \\
$10^{2} \operatorname{Im}\left(\beta_{0}\right)$ & $1.32(2)$ & $1.33(3)$ & $1.33(3)$ & $1.28(5)$ & $0.98(12)$ \\
\hline $\operatorname{Re}\left(\beta_{0}\right) ; L_{s}=10$ & $5.1892(3)$ & $5.1865(3)$ & $5.1821(3)$ & $5.1758(8)$ & $5.1751(11)$ \\
$10^{3} \operatorname{Im}\left(\beta_{0}\right)$ & $7.27(14)$ & $7.26(15)$ & $7.33(22)$ & $6.44(74)$ & $5.29(76)$ \\
\hline $\operatorname{Re}\left(\beta_{0}\right) ; L_{s}=12$ & $5.1888(2)$ & $5.1861(2)$ & $5.1817(3)$ & $5.1768(4)$ & $5.1739(6)$ \\
$10^{3} \operatorname{Im}\left(\beta_{0}\right)$ & $4.95(12)$ & $4.95(13)$ & $4.88(20)$ & $4.16(71)$ & $2.07(74)$ \\
\hline $\operatorname{Re}\left(\beta_{0}\right) ; L_{s} \rightarrow \infty$ & $5.1893(3)$ & $5.1866(3)$ & $5.1822(3)$ & $5.1769(5)$ & $5.1745(6)$ \\
$10^{3} \operatorname{Im}\left(\beta_{0}\right)$ & $2.12(14)$ & $2.12(16)$ & $2.14(23)$ & $1.77(65)$ & $-0.39(77)$ \\
\hline \hline$\beta$ & $m_{u, d}$ & $m_{\pi}$ & $m_{K}$ & $m_{\rho}$ & $R_{0}$ \\
\hline 5.09 & 0.02 & $0.3555(1)$ & $0.8948(2)$ & $1.361(9)$ & $1.58(2)$ \\
5.09 & 0.04 & $0.4978(1)$ & $0.9235(1)$ & $1.391(4)$ & $1.58(1)$ \\
5.09 & 0.06 & $0.6044(1)$ & $0.9511(1)$ & $1.423(4)$ & $1.57(1)$ \\
\hline 5.16 & 0.02 & $0.3630(2)$ & $0.9061(3)$ & $1.306(10)$ & $1.73(3)$ \\
5.16 & 0.04 & $0.5063(2)$ & $0.9335(2)$ & $1.344(8)$ & $1.67(1)$ \\
5.16 & 0.06 & $0.6129(1)$ & $0.9603(1)$ & $1.389(4)$ & $1.64(1)$ \\
\hline 5.19 & 0.02 & $0.3674(1)$ & $0.9122(3)$ & $1.287(8)$ & $1.77(2)$ \\
5.19 & 0.04 & $0.5063(1)$ & $0.9337(2)$ & $1.325(6)$ & $1.72(1)$ \\
5.19 & 0.06 & $0.6130(1)$ & $0.9604(1)$ & $1.366(2)$ & $1.70(1)$ \\
\hline
\end{tabular}

pion to rho mass ratio: $0.188(2)$ (physical value is 0.18 ) pion to $K$ mass ratio: $0.267(1)$ (physical value is 0.28 ) 
- $T$ as a function of the baryonic chemical potential $\mu_{B}$

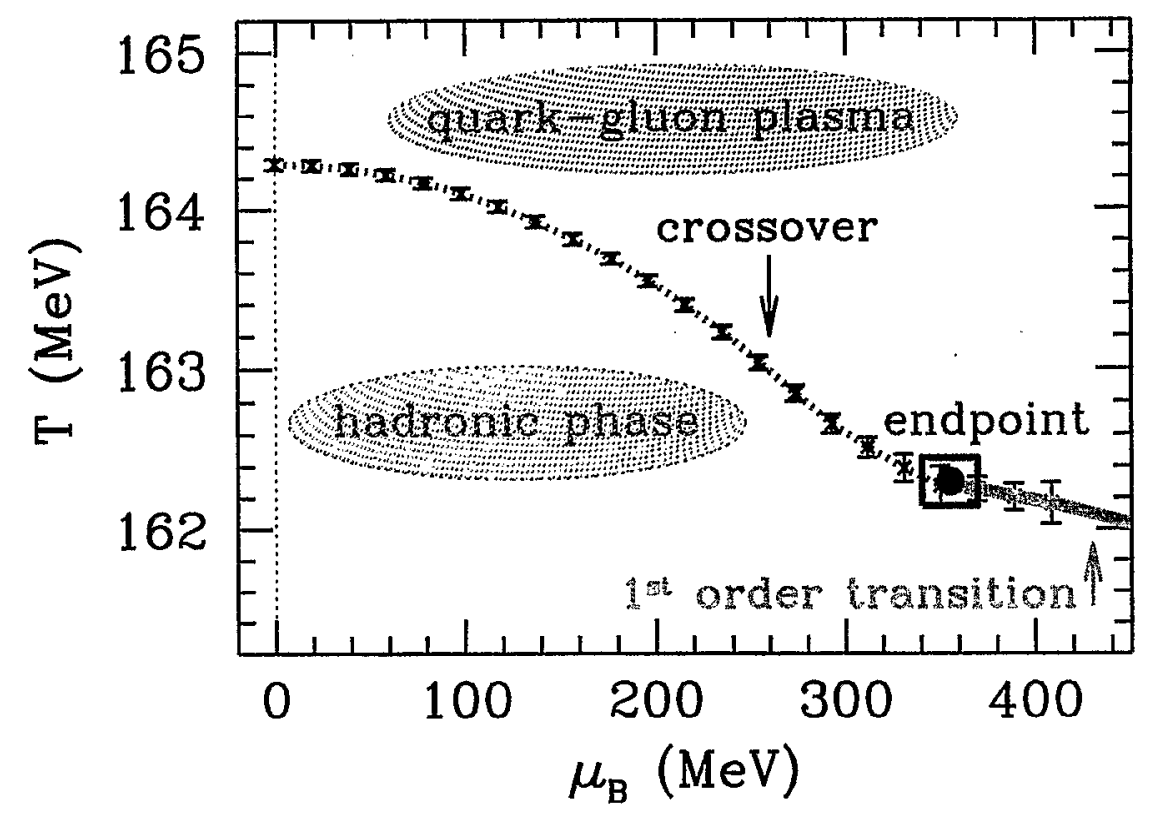

- lattice result for physical quark masses at $L_{t}=4$

$$
\begin{gathered}
\text { endpoint: } T_{E}=162 \pm 2 \mathrm{MeV}, \mu_{E}=360 \pm 40 \mathrm{MeV} \\
\text { at } \mu_{B}=0 \text { transition temperature: } T_{C}=164 \pm 2 \mathrm{MeV} . \\
T / T_{C}=1-C \mu_{B}^{2} / T_{c}^{2} \text { wit } C=0.0032(1)
\end{gathered}
$$

as expected: $E$ moves closer to $\mu=0, T_{c}$ decreases 


\title{
Taylor expansion at non-zero density and \\ the chiral critical end-point at 3 flavor QCD
}

C. Schmidt ${ }^{a}$ with C.R. Allton, S. Ejiric, S.J. Hands, O. Kaczmarek, F. Karsch, and E. Laermann ${ }^{c}$

${ }^{a}$ Fachbereich Physik, Bergische Universität Wuppertal, D-42097 Wuppertal, Germany.

b Department of Physics, University of Wales Swansea, Singleton Park, Swansea SA2 8PP, U.K.

c Fakultät für Physik, Universität Bielefeld, D-33615 Bielefeld, Germany.

\begin{abstract}
We present results of a QCD Monte Carlo simulation with 3 continuum flavors of p4-improved staggered fermions with mass $m / T=0.02$. Using a multi-parameter reweighting method, we explore the phase diagram of the chiral transition in dependence of temperature, quark mass and quark chemical potential. Based on estimates of the Binder Cumulant and a correlation analysis of different Reweighting operators we discuss the chemical potential and strange quark mass dependence of the critical end-point on the transition line.
\end{abstract}




\section{1) Lattice $Q C D$ at non-zero Density}

\section{- Taylor expansion}

Taylor-expanded reweighting up to order $\mathcal{O}\left(\omega^{2}\right), \omega=(m, \mu)$ :

$$
\begin{aligned}
\langle O\rangle_{\omega, \beta}= & \frac{\left\langle\left(O_{0}+O_{1} \Delta \omega+O_{2}(\Delta \omega)^{2}\right) \exp \left\{R_{1} \Delta \omega+R_{2}(\Delta \omega)^{2}\right\} \exp \left\{\Delta S_{G}\right\}\right\rangle_{\omega_{0}, \beta_{0}}}{\left\langle\exp \left\{R_{1} \Delta \omega+R_{2}(\Delta \omega)^{2}\right\} \exp \left\{\Delta S_{G}\right\}\right\rangle_{\omega_{0}, \beta_{0}}} \\
& +\mathcal{O}\left((\Delta \omega)^{3}\right)
\end{aligned}
$$

N

Bielefeld-Swansea, Phys. Rev. D66 (2002) 074507.

$$
\begin{aligned}
R_{1} \Delta \omega= & \left.\frac{n_{f}}{4} \frac{\partial \ln \operatorname{det} M}{\partial m}\right|_{\Delta m=0} \Delta m+\left.\frac{n_{f}}{4} \frac{\partial \ln \operatorname{det} M}{\partial \mu}\right|_{\mu=0} \mu \\
\equiv & R_{10}+R_{01} \\
R_{2}(\Delta \omega)^{2}= & \left.\frac{n_{f}}{8} \frac{\partial^{2} \ln \operatorname{det} M}{\partial m^{2}}\right|_{\Delta m=0}(\Delta m)^{2}+\left.\frac{n_{f}}{8} \frac{\partial^{2} \ln \operatorname{det} M}{\partial \mu^{2}}\right|_{\mu=0} \mu^{2} \\
& \left.\frac{n_{f}}{4} \frac{\partial^{2} \ln \operatorname{det} M}{\partial m \partial \mu}\right|_{\Delta m=\mu=0} \Delta m \mu \\
\equiv & R_{20}+R_{02}+R_{11}
\end{aligned}
$$




\section{2) Investigation of the Phase Diagram}

- Determination of the 3-flavor chiral

critical point at $\mu=0$

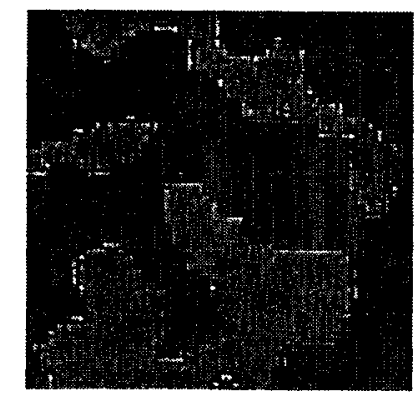

Susceptibilities:
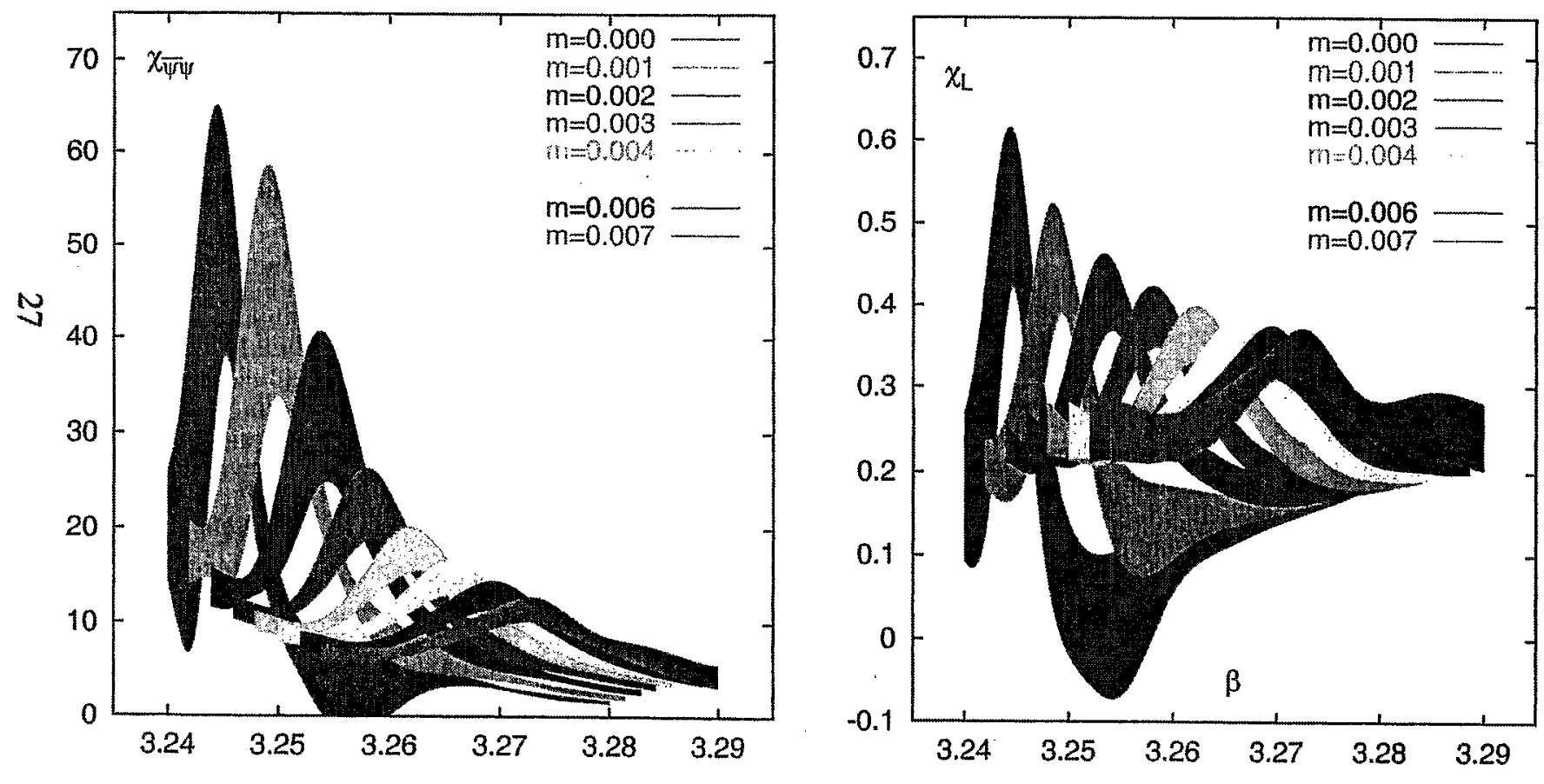

- pronounced

peak in $\chi_{\bar{\psi} \psi}, \chi_{L}$

- $n$-dependence

of the peak

position

tincreasing peak

height with

$m \rightarrow 0$

- finite volume

effect

Simulations at quark mass $m=0.005\left(m_{q} \simeq 172 \mathrm{MeV}\right)$

$\longrightarrow$ mass-reweighting, $R: \mathcal{O}\left((\Delta m)^{2}\right)$

- truncation

error 


\section{2) Investigation of the Phase Diagram}

- Determination of the 3-flavor chiral

$$
\text { critical point at } \mu=0
$$

\section{Results from standard-action:}
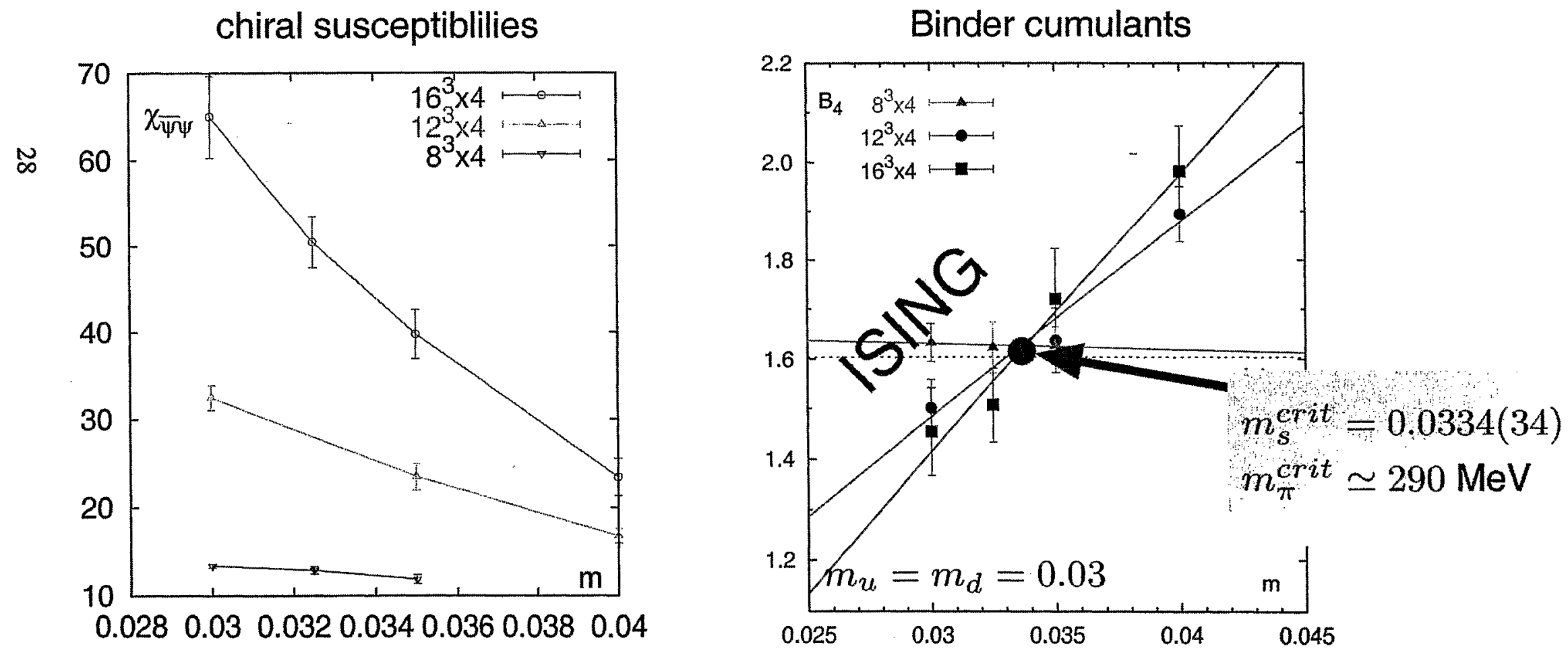

$\Longrightarrow$ standard-action enables high statistics 


\title{
2) Investigation of the Phase Diagram
}

- Determination of the 3-flavor chiral critical point at $\mu=0$
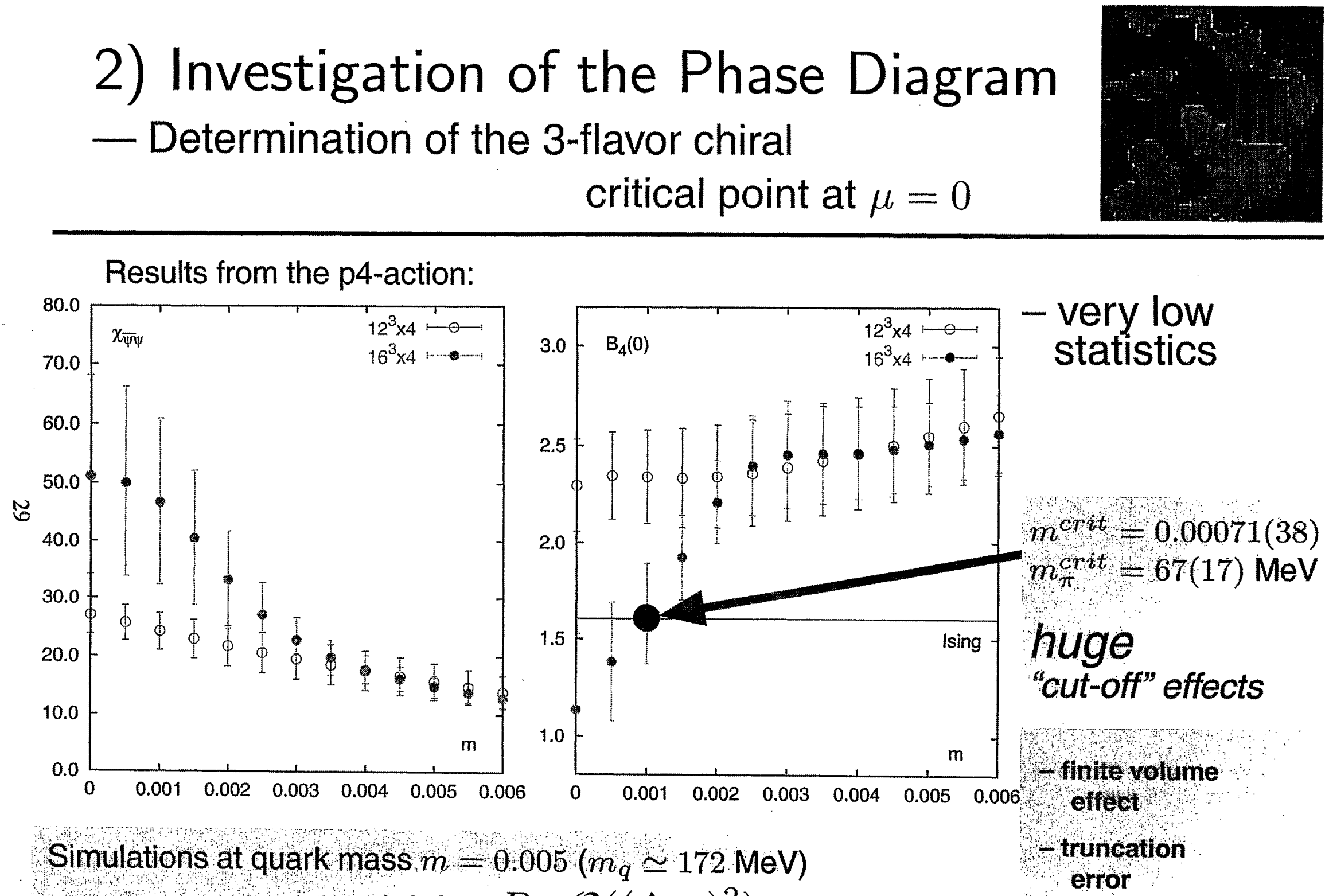

Simulations at quark mass $m=0.005\left(m_{q} \simeq 172 \mathrm{MeV}\right)$ $\Longrightarrow$ mass reweighting, $R: \mathcal{O}\left((\Delta m)^{2}\right)$

\author{
error
}




\section{3) Investigation of the Phase Diagram - Approximation of the critical surface in the vicinity of the 3-flavor critical point}

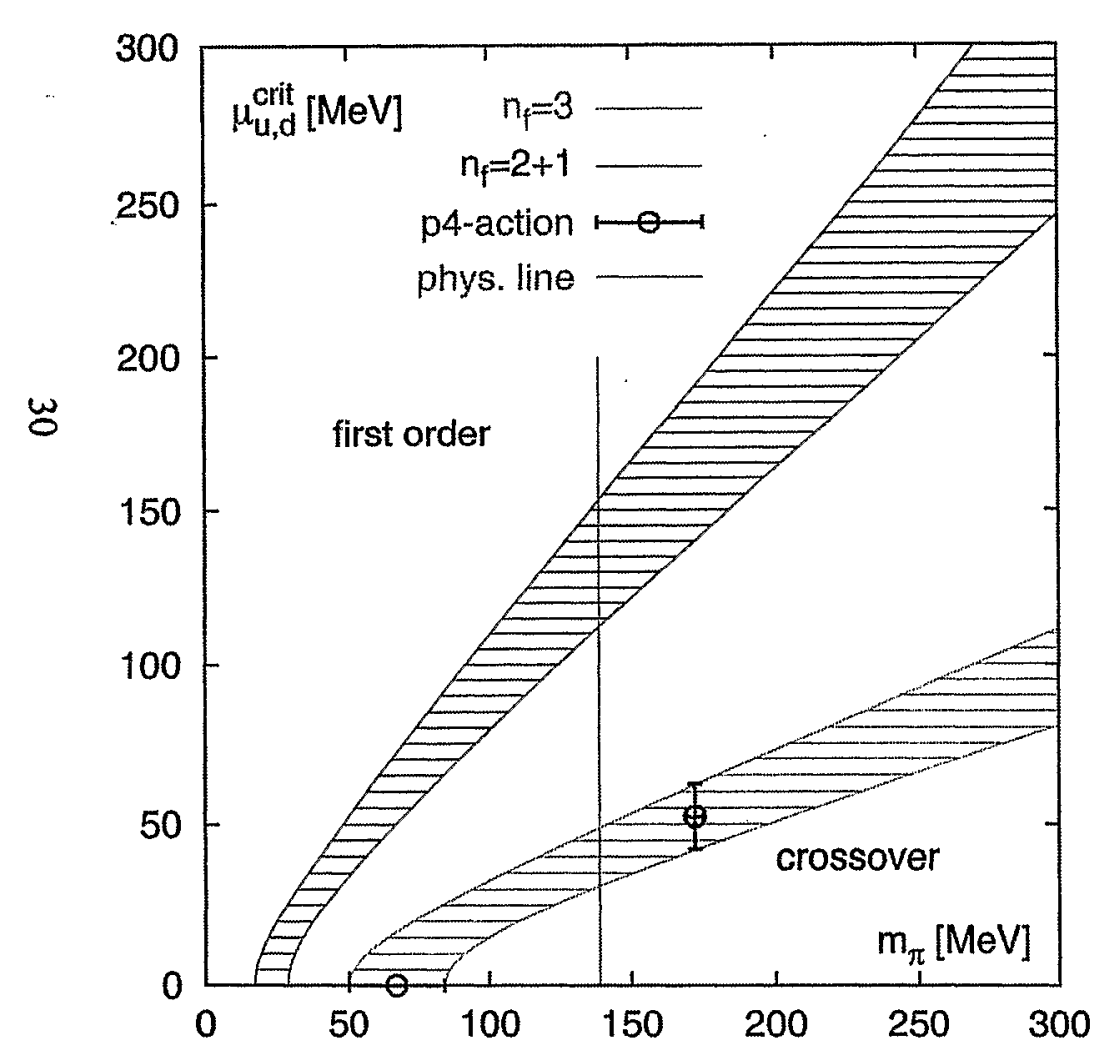

The critical surface:

$\rightarrow$ in the $\left(m_{u, d}, m_{s}\right)$-plane:

$$
m_{s}^{c r i t}=3 m_{u, d}^{c r i t}-2 m_{u, d}
$$

$\rightarrow$ chiral perturbation theory:

$$
m_{K}^{2} / m_{\pi}^{2}=\left(m_{u, d}+m_{s}\right) / 2 m_{u, d}
$$

$\rightarrow$ in the $\left(m_{\pi}^{2}, m_{K}^{2}\right)$-plane:

$$
\left(m_{K}^{c r i t}\right)^{2}=\frac{3}{2}\left(m_{\pi}^{c r i t}\right)^{2}-\frac{1}{2} m_{\pi}^{2}
$$

$\rightarrow$ quadratic interpolation of the 3-flavor critical points:

$$
\left(m_{\pi}^{c r i t}\right)^{2}\left[\mu_{q}\right]=\left(m_{\pi}^{c r i t}\right)^{2}[0]+A \mu_{q}^{2}
$$

$\Longrightarrow$ surface defined by (1) and (2) 
February 2004

\title{
QCD Thermodynamics from Imaginary $\mu$
}

\author{
Owe Philipsen (University of Sussex) \\ with Philippe de Forcrand (ETH/CERN)
}

Motivation

- Imaginary chemical potential

- "Analyticity" of the pseudo-critical line

- $T_{c}(\mu): N_{f}=2,3,4$ results and comparison with other methods

- Critical endpoint and its quark mass dependence for $N_{f}=3$

- Outlook on $N_{f}=2+1$

- Conclusions 
Analyticity of the (pseudo-) criticall line with P. de Forcrand phase transition from maximum of susceptibilites:

$$
\chi(\beta, \bar{\mu}, V)=V N_{t}\left\langle(\mathcal{O}-\langle\mathcal{O}\rangle)^{2}\right\rangle, \quad \mathcal{O} \in\{\text { plaq, } \bar{\psi} \psi,|P(x)|\}
$$

finite volume: suscept. finite and analytic in all cases

- Location of transition:

Critical line $\beta_{c}(\bar{\mu})$ defined by peak $\chi_{\max } \equiv \chi\left(\bar{\mu}_{c}, \beta_{c}\right)$

$$
\left.\frac{\partial \chi}{\partial \beta}\right|_{\bar{\mu}_{c}, \beta_{c}}=0,\left.\quad \frac{\partial^{2} \chi}{\partial \beta^{2}}\right|_{\bar{\mu}_{c, \beta_{c}}}<0 .
$$

Implicit function theorem:

$\chi(\beta, \bar{\mu})$ analytic $\Rightarrow \beta_{c}(\bar{\mu})$ analytic!

symmetries: $\chi(\bar{\mu})=\chi(-\bar{\mu}) \Rightarrow \beta_{c}(\bar{\mu})=\beta_{c}(-\bar{\mu})$

$$
\beta_{c}(\bar{\mu})=\sum_{n} c_{n}(a \bar{\mu})^{2 n}
$$


$N_{f}=3$ results, $T_{c}(\mu, m):$

de Forrond of

vary quark masses $m$, much more stats.

check for NLO terms in Taylor series:

$$
\beta_{c}(a \mu, a m)=\sum_{k, l=0} c_{k l}(a \mu)^{2 k}(a m)^{l}
$$

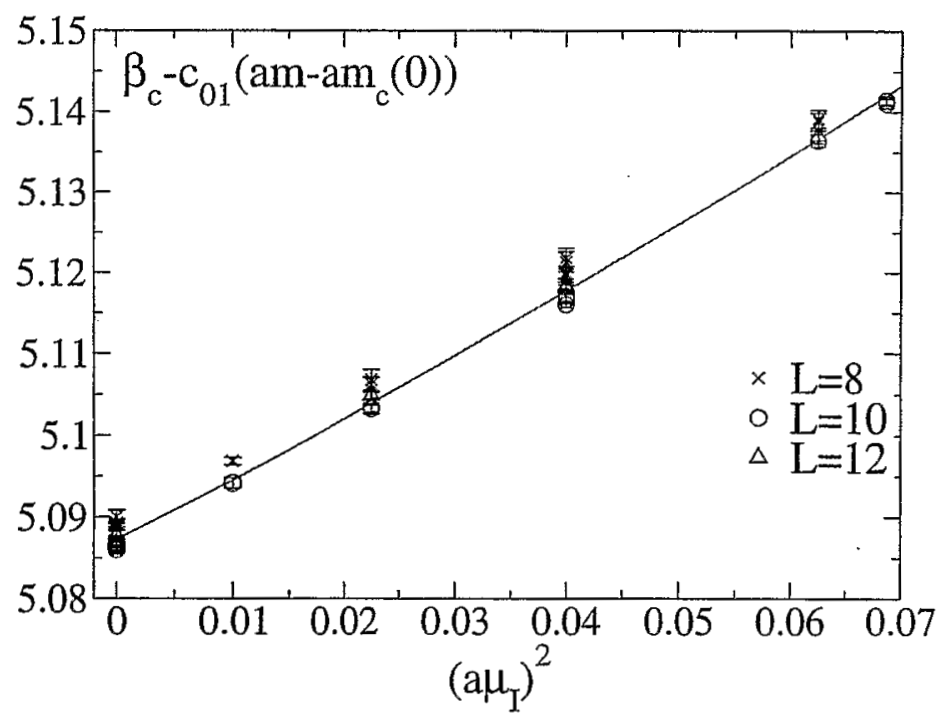

$\Rightarrow$ evidence for $\mu^{4} ! \quad$ no evidence for $\mathcal{O}\left(m^{2}, \mu^{2} m\right)$

$$
\begin{aligned}
& \frac{T_{c}(\mu, m)}{T_{c}\left(\mu=0, m_{c}(0)\right)}=1-1.94(2)\left(\frac{m-m_{c}(0)}{\pi T_{c}}\right) \\
& +0.602(9)\left(\frac{\mu}{\pi T_{c}(0, m)}\right)^{2}+0.23(9)\left(\frac{\mu}{\pi T_{c}(0, m)}\right)^{4}
\end{aligned}
$$


The critical endpoint Phase diag. 3d: $(T, \mu, m)$

-confined/deconfined $\Rightarrow$ pseudo-crit. surface $T_{c}(\mu, m)$

On this surface,

-1.O./crossover $\Rightarrow$ line of crit.points $T^{*}(\mu)=T_{c}\left(\mu, m_{c}(\mu)\right)$

Project on $(T, \mu)$ :

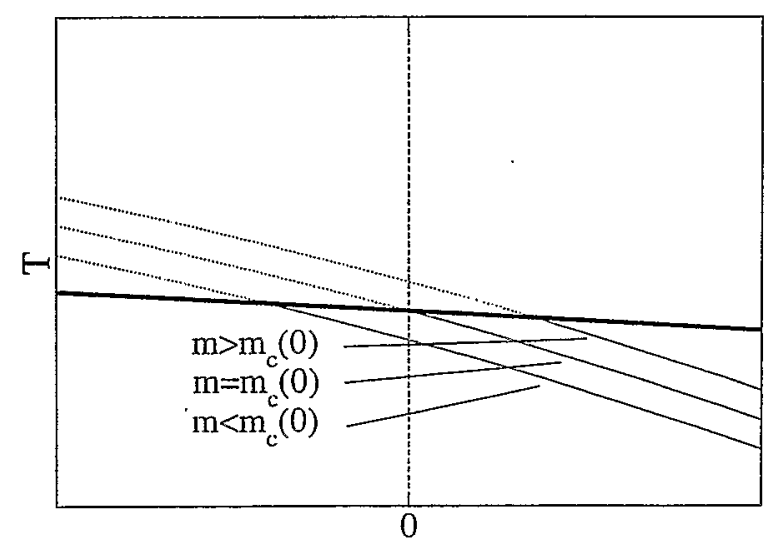

Project on $(m, \mu)$ :

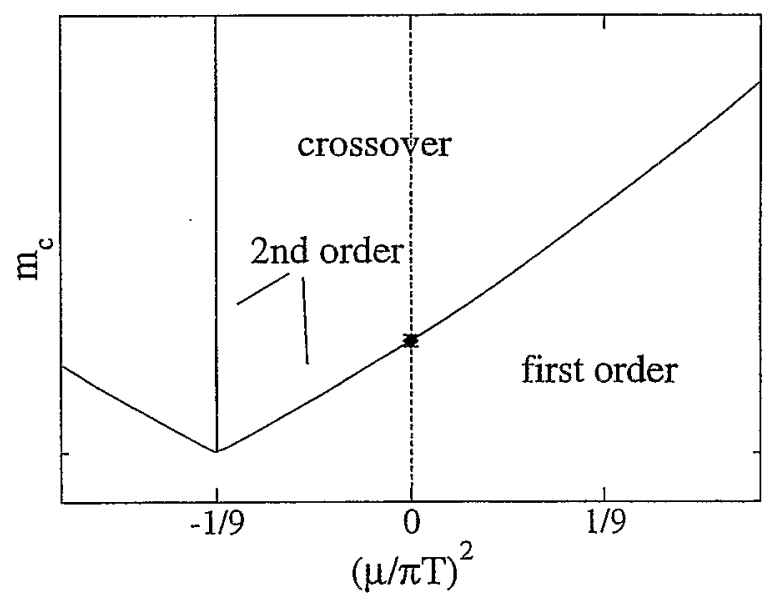

$m=0 \Rightarrow$ true chiral phase transition

Expect: $\quad \frac{m_{c}(\mu)}{m_{c}(\mu=0)}=1+c_{1}\left(\frac{\mu}{\pi T}\right)^{2} \Rightarrow c_{1} \leq 9$ 


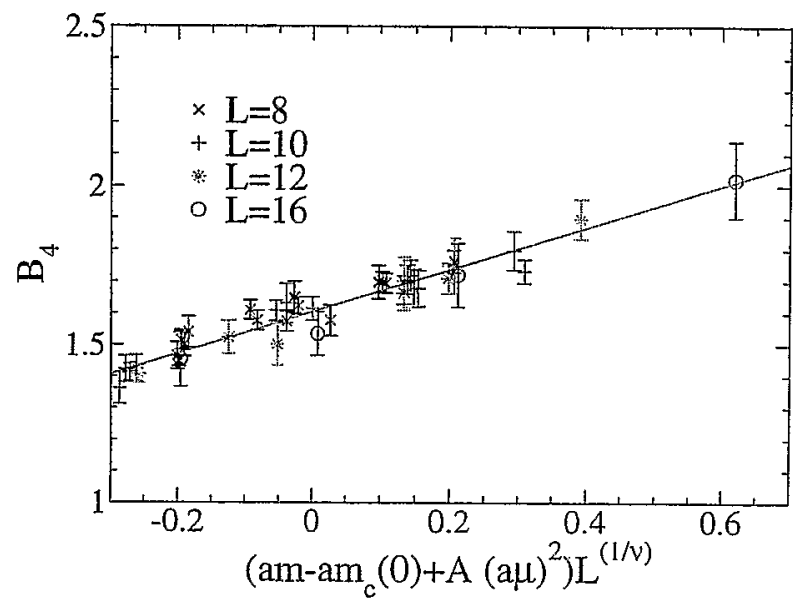

Critical quark mass, $\mu_{c}$ :

$$
\Rightarrow \frac{m_{c}(\mu)}{m_{c}(\mu=0)}=1+0.84(36)\left(\frac{\mu}{\pi T}\right)^{2}+\ldots
$$

\section{tentative comparison}

Taylor expanded rew., improved action: $c_{1} \sim 290 \ldots .2500$ Allon et al.

$\Rightarrow$ inconsistent, our critical point further out 


\section{Preliminary results:}

$m_{u, d}=0.025$ (Fodor,Katz)

We find $0.031 \lesssim m_{s}^{c}(0) \lesssim 0.45$

$$
\frac{m_{c}(\mu)}{m_{c}(\mu=0)}=1+c_{1}\left(\frac{\mu}{\pi T}\right)^{2}
$$

N.B: $\quad$ constraint on $c_{1}$ remains valid for $N_{f}=2+1$

$\Rightarrow m_{s}^{c}\left(\mu^{2}\right)-\mu^{2}$ diagram:

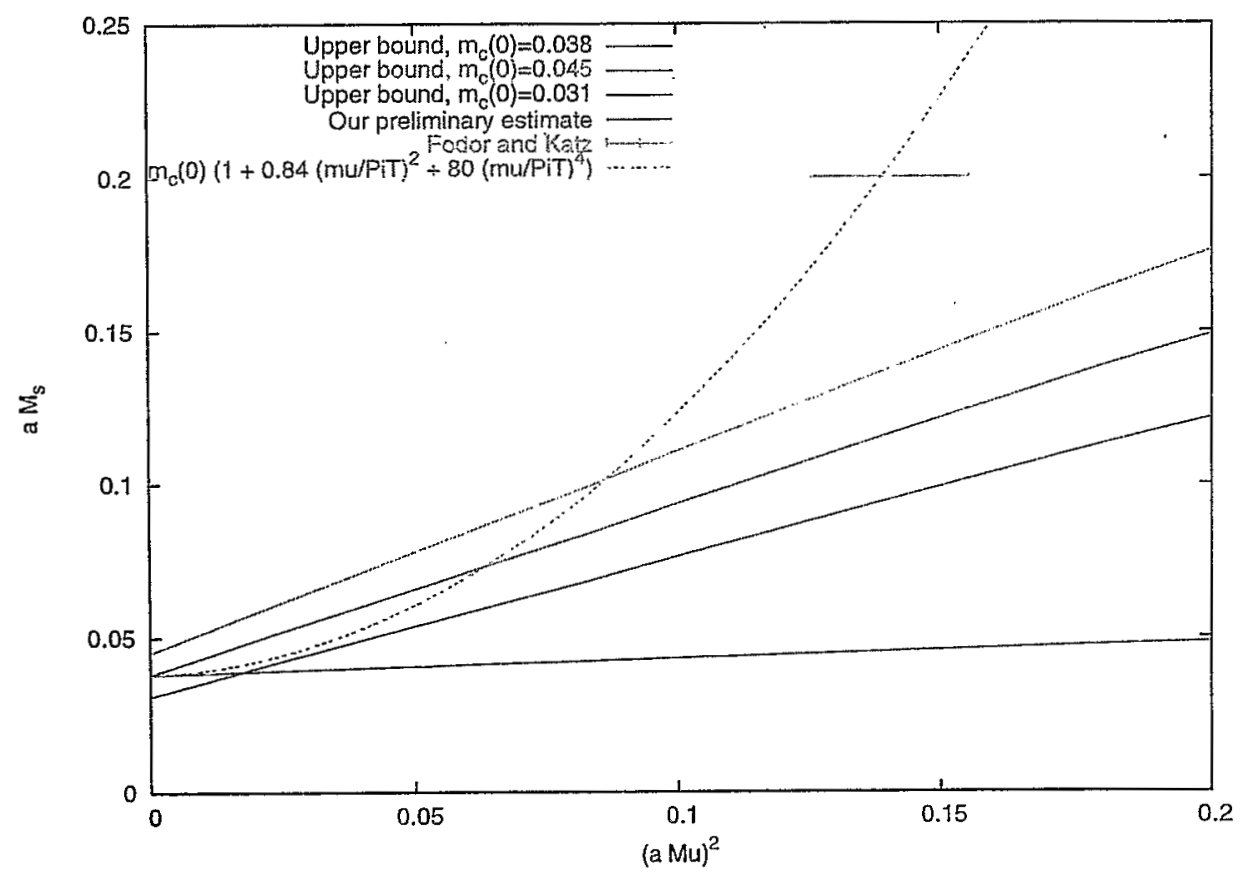

$\Rightarrow$ The only way to reconcile these are strong non-linearities! 
The QCD equation of state at finite $T$ and $\mu$ : lattice result

$$
\text { Sándor D. Katz (University of Wuppertal) }
$$

Z. Fodor, ȘDK, K.K. Szabó, hep-lat/0208078

F. Csikor, G.I. Egri, Z. Fodor, SDK, K.K. Szabó, A.I. Tóth, hep-lat/0401016

$\dot{v}$

1. Introduction

2. Line of constant physics

3. Equation of state at vanishing chemical potential

4. Overlap improving multi-parameter reweighting

5. Goodness of reweighting

6. Equation of state at non-vanishing chemical potential

7. Summary 
Equation of state along the line of constant physics (LCP) energy density $(\epsilon)$ and pressure $(p)$ from partition function:

$$
\epsilon(T)=\frac{T^{2}}{V} \frac{\partial(\log Z)}{\partial T} \quad p(T)=T \frac{\partial(\log Z)}{\partial V} .
$$

$T, V$ are varied by $a$, take $\frac{\partial \beta}{\partial a}$ and $\frac{\partial\left(a m_{i}\right)}{\partial a}=m_{i}$ on the LCP

$$
\frac{\epsilon-3 p}{T^{4}}=-\frac{L_{t}^{3}}{L_{s}^{3}} a \frac{d(\log Z)}{d a}=
$$

$$
-L_{t}^{4}\left[a \frac{\partial \beta}{\partial a}<\mathrm{P}>+a \frac{\partial\left(a m_{u}\right)}{\partial a}<\bar{u} u>+a \frac{\partial\left(a m_{s}\right)}{\partial a}<\bar{s} s>\right]
$$

the pressure $(p \propto \log [Z])$ along the LCP by the integral method:

$$
\begin{gathered}
\frac{p}{T^{4}}=L_{t}^{4} \int d(\beta, m \cdot a)\left(\frac{\partial(\log Z)}{\partial \beta}, \frac{\partial(\log Z)}{\partial(m \cdot a)}\right)= \\
\left.L_{t}^{4} \int d \beta\left[\langle\mathrm{P}\rangle+m_{u} \frac{\partial a}{\partial \beta}\langle\bar{u} u\rangle+m_{s} \frac{\partial a}{\partial \beta}<\bar{s} s\right\rangle\right]
\end{gathered}
$$


Equation of state at vanishing chemical potential
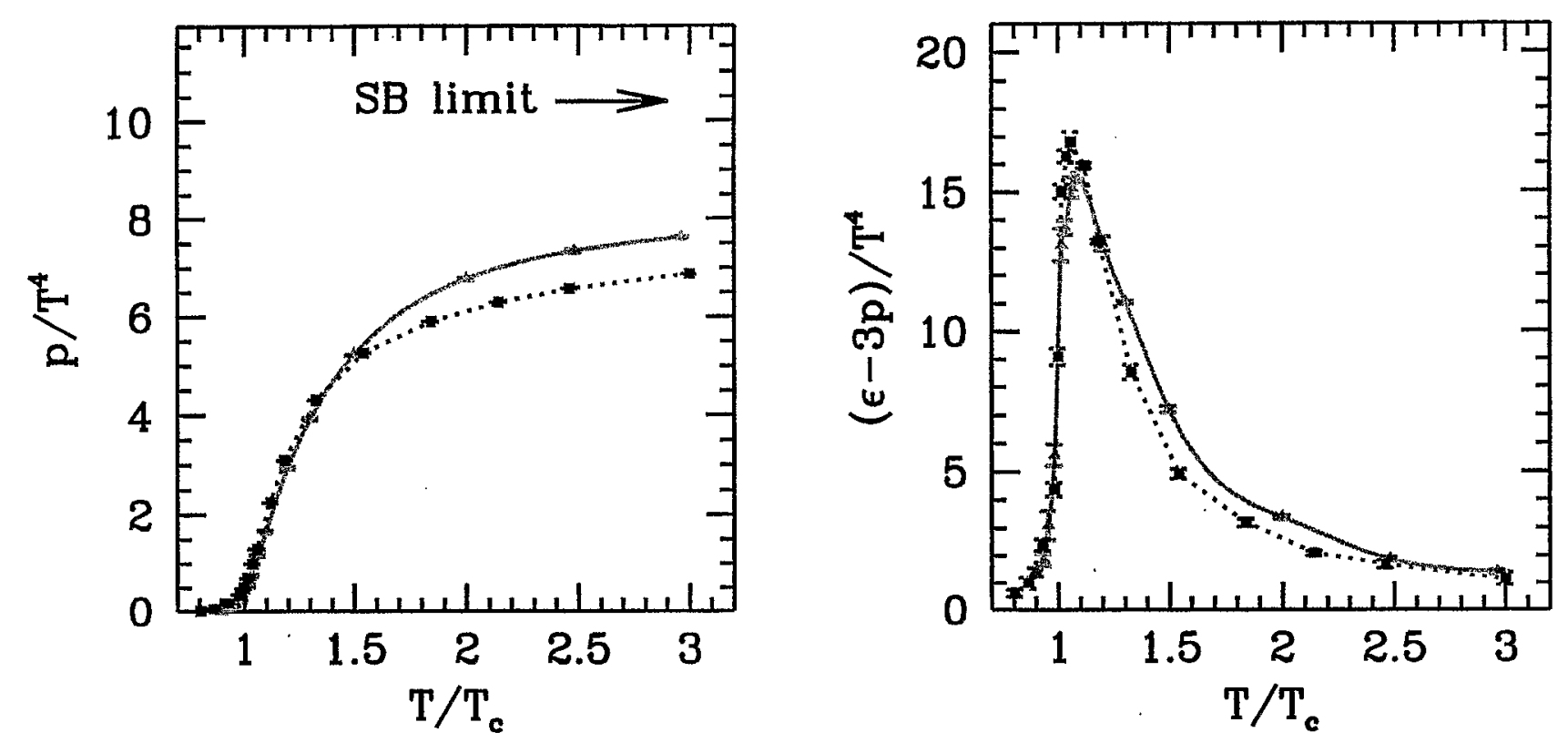

differences in $p, \epsilon-3 p$ values

following the LCP or using the "non-LCP approach" (fixed $m \cdot a$ ) setting the scales, $\beta$ functions are ambiguous

the value of the pressure is independent of the integration path $p \approx 20-25 \%$ below the Stefan-Boltzmann limit at $T \approx 3 T_{c}$ 


\section{Reliability of reveighting}

(see also [Ejiri, 2004] for other possibilities)

Idea:

From the original configurations $\Phi_{i}$ generate a new set $\Phi_{i}^{\prime}$ with a Metropolis-like accept reject:

$$
\begin{aligned}
& \Phi_{1}^{\prime}=\Phi_{1} \\
& \Phi_{i}^{\prime}=\Phi_{i} \text { with probability } \max \left(1, w_{i} / w_{i-1}^{\prime}\right) \\
& \Phi_{i-1}^{\prime} \text { otherwise }
\end{aligned}
$$

$\Phi^{\prime}$ has the desired distribution $\rightarrow$ errors can be computed

Problems:

- $\Phi^{\prime}$ may not be thermalized

- what to do with complex weights (use $\left|w_{i}\right|$ or $\left|\operatorname{Re} w_{i}\right|$ )

extreme reweighting: $\Phi^{\prime}$ only changes when $w_{i}>w_{i-1}$. after the largest weight all $\Phi_{i}^{\prime}$ the same $\rightarrow$ zero information $\rightarrow$ use always $\Phi_{i}^{\prime}$-s after the largest weight only 
- direct test: chiral condensate of $n_{f}=4$ dynamical QCD $m_{q}=0.05$ staggered fermions, imaginary $\mu$ on $4 \cdot 6^{3}$ lattices
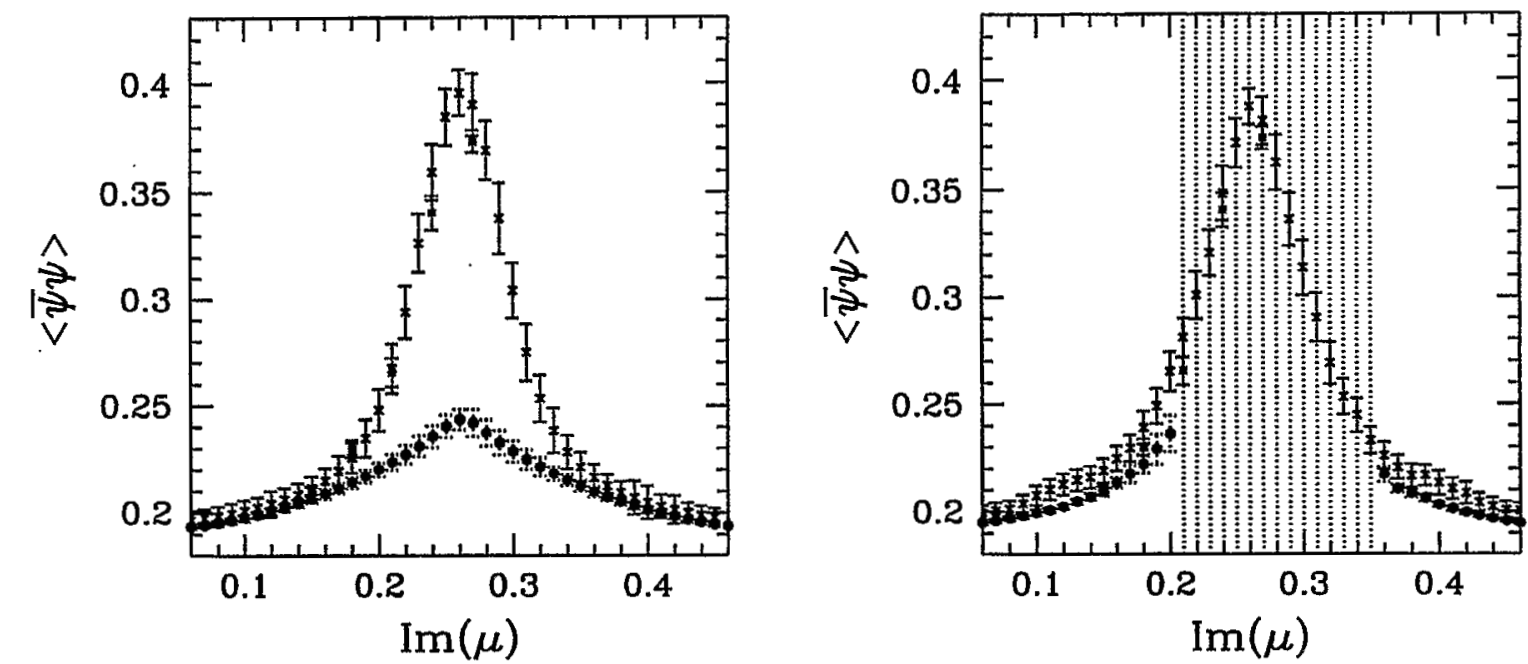

Glasgow-method: based on an ensemble of the high-T phase high- $\mu$ phase does not overlap with the states of interest

error estimate: based on Metropolis technique using the weights naturally works for "continuously connected" samples for first order transitions the method needs all sectors (e.g. $Z_{3}$ ) realistic only for huge samples (no inherent definition) 
- equation of state at finite chemical potential upto $\mu_{q} \approx T_{\mathcal{C}}$ difference between the $\mu=0$ and $\mu \neq 0$ pressure values chemical potential for $\mu_{B} \approx 100,210,330,410,530 \mathrm{MeV}$
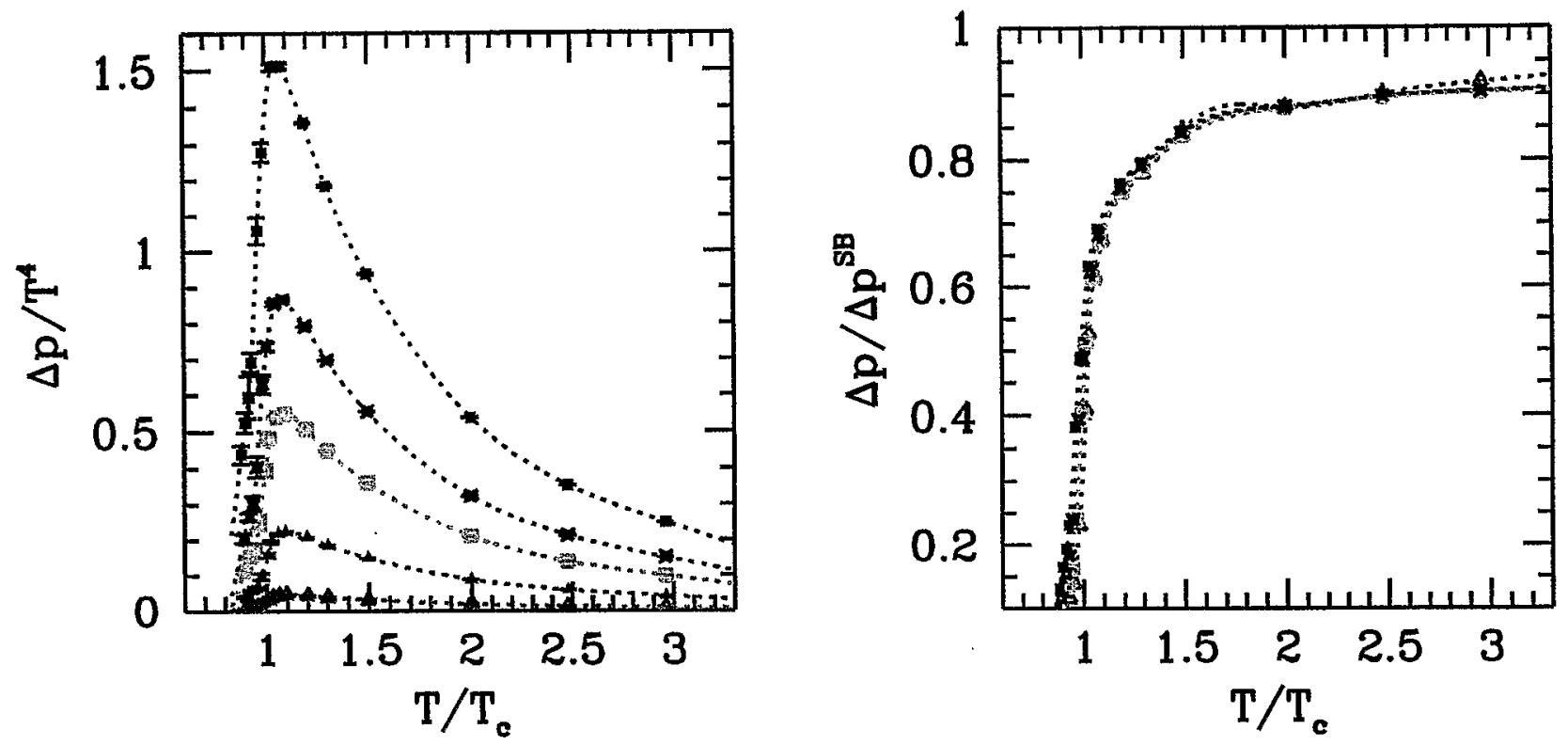

normalize with the leading order $\mu$ dependence: $\triangle p^{S B}$ almost universal temperature dependence for $\triangle p / \triangle p^{S B}$ 


\title{
QCD thermodynamics at non-zero $\mu$
}

\author{
Shinji Ejiri \\ Fakultät für Physik, Universität Bielefeld, D-33615 Bielefeld, Germany.
}

\begin{abstract}
It is important to study QCD at high temperature and high density by numerical simulations of lattice QCD. In particular, studies of the equation of state (EoS) can provide basic input for the analysis of the experimental signatures for QGP formation. We extend studies of the EoS to non-zero baryon number density. The simulation at nonzero baryon density has been known to be difficult. However, studies based on a Taylor expansion with respect to chemical potential $\mu_{\mathrm{q}}$ turned out to be an efficient technique to investigate the low density regime, interesting for heavy-ion collisions. We discuss the EoS for 2 flavor QCD at non-zero temperature and density. Derivatives of $\ln \mathcal{Z}$ with respect to $\mu_{\mathrm{q}}$ are calculated up to sixth order. From this Taylor series, the pressure, quark number density and associated susceptibilities are estimated as functions of temperature and $\mu_{\mathrm{q}}$. It is found that the fluctuations in the quark number density increase in the vicinity of the phase transition temperature and the susceptibilities start to develop a pronounced peak as $\mu_{q}$ is increased. This suggests the presence of a critical endpoint in the $\left(\mu_{q}, T\right)$ plane.
\end{abstract}




\section{Derivatives of pressure}
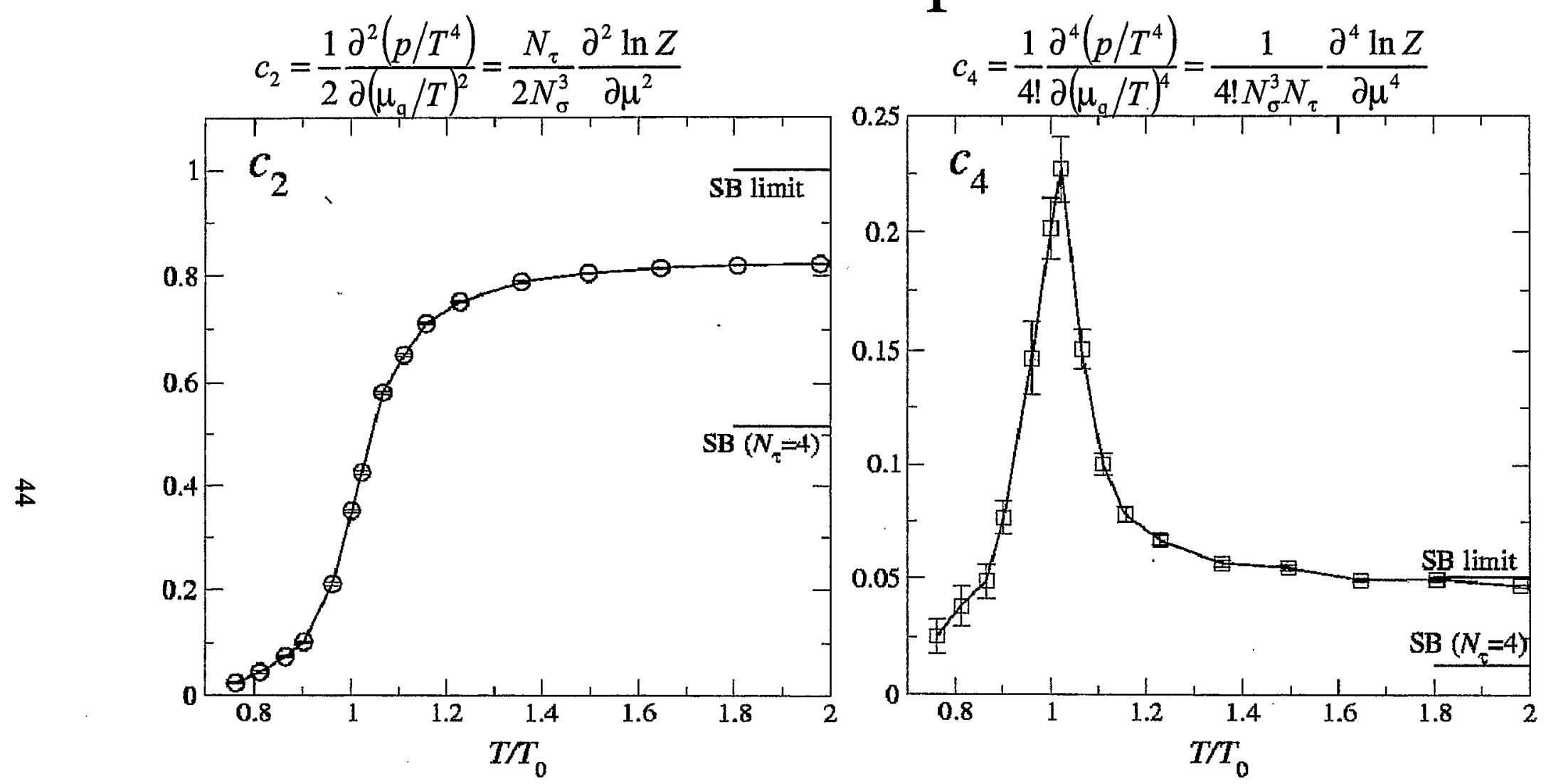

- Both terms are exponentially small in low $T$ phase and approach SB limit in the high $T$ limit.

- $\mathrm{O}\left(\mu^{4}\right)$ term: Peak around critical $T$.

$$
T_{0}=T_{C}(\mu=0) \approx 170 \mathrm{MeV}
$$




\section{Hadron resonance gas or QGP gas}

- At $T<T_{\mathrm{c}}, C_{4} / C_{2}$ is consistent with the prediction from

hadron resonance gas model (non-interacting baryon gas).

[Karsch-Redlich-Tawfik, hep-ph/0306208]

古

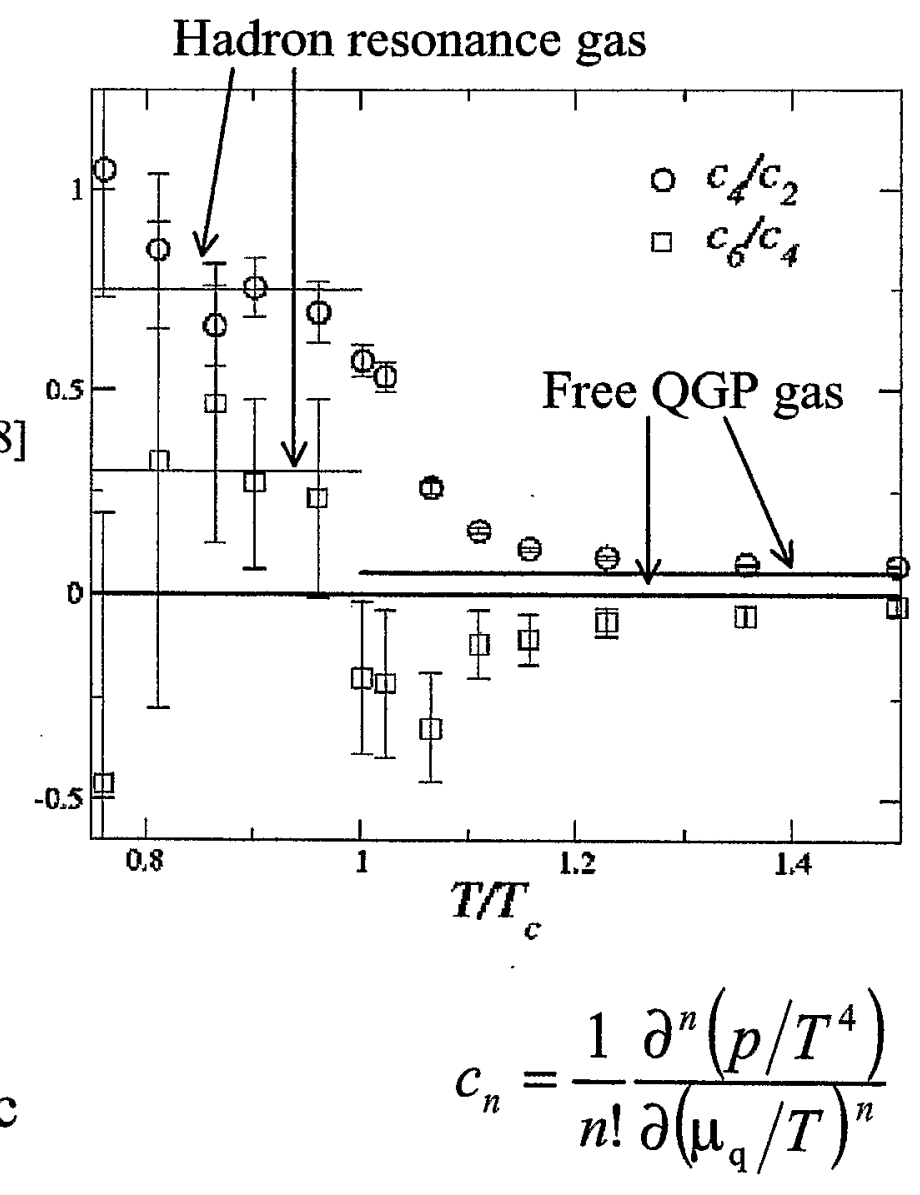

- At $T>T_{\mathrm{c}}, C_{4} / C_{2}$ approaches the value of a free QGP gas.

$\Rightarrow$ the sharp peak of $C 4$ at $T_{\mathrm{c}}$

$$
\frac{p}{T^{4}}=F(T) \cosh \left(\frac{3 \mu_{q}}{T}\right)
$$




\section{Difference of pressure for $\mu>0$ from $\mu=0$}

$$
\frac{p}{T^{4}}(\mu)=\frac{p}{T^{4}}(0)+c_{2}\left(\frac{\mu_{\mathrm{q}}}{T}\right)^{2}+c_{4}\left(\frac{\mu_{\mathrm{q}}}{T}\right)^{4}+c_{6}\left(\frac{\mu_{\mathrm{q}}}{T}\right)^{6}+O\left(\mu_{\mathrm{q}}^{8}\right) \quad \quad \mu_{\mathrm{q}} / T \text { : constant }
$$

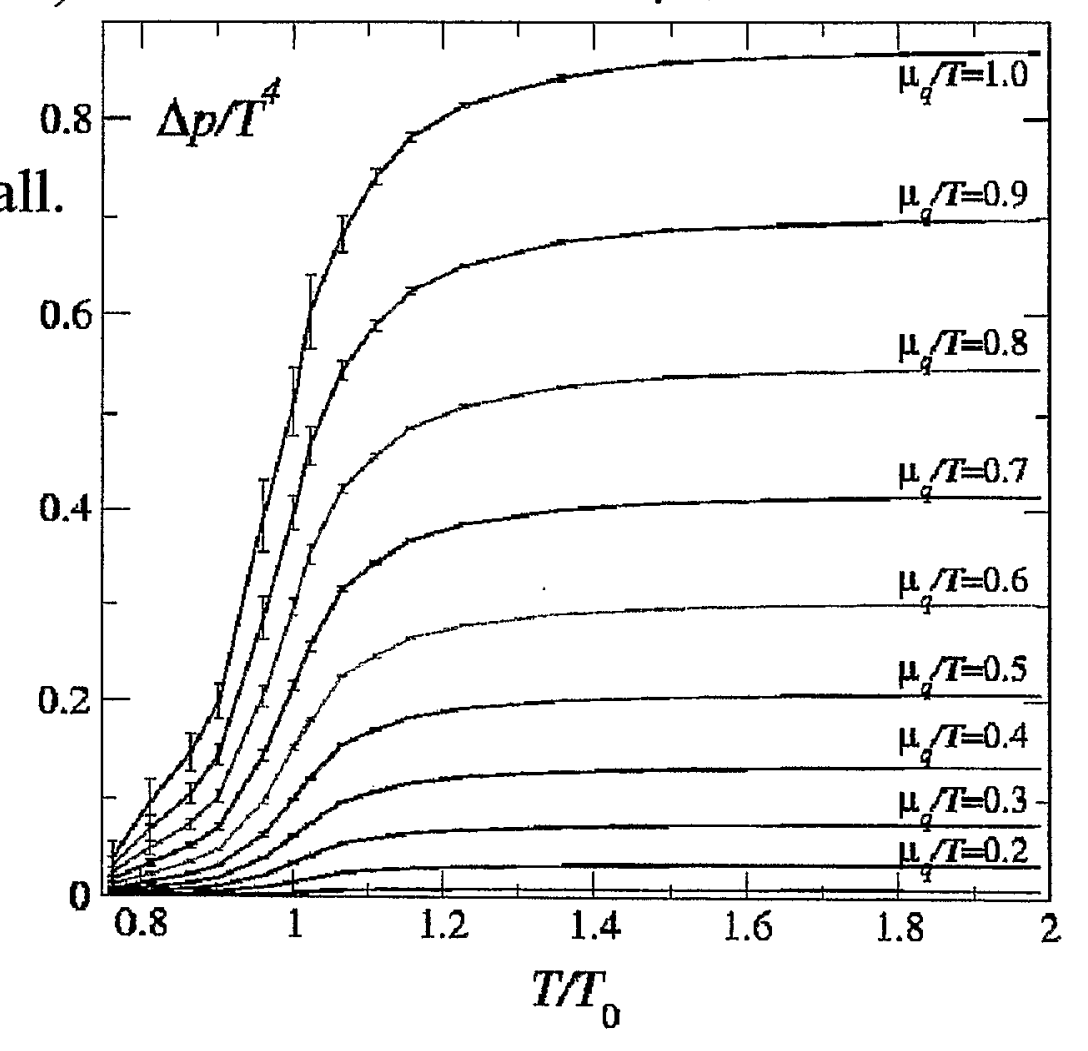

- Qualitatively agree with [Fodor-Katz-Szabo, hep-lat/0208078].

$$
T_{0}=T_{C}(\mu=0) \approx 170 \mathrm{MeV}
$$




\section{Quark number susceptibility}

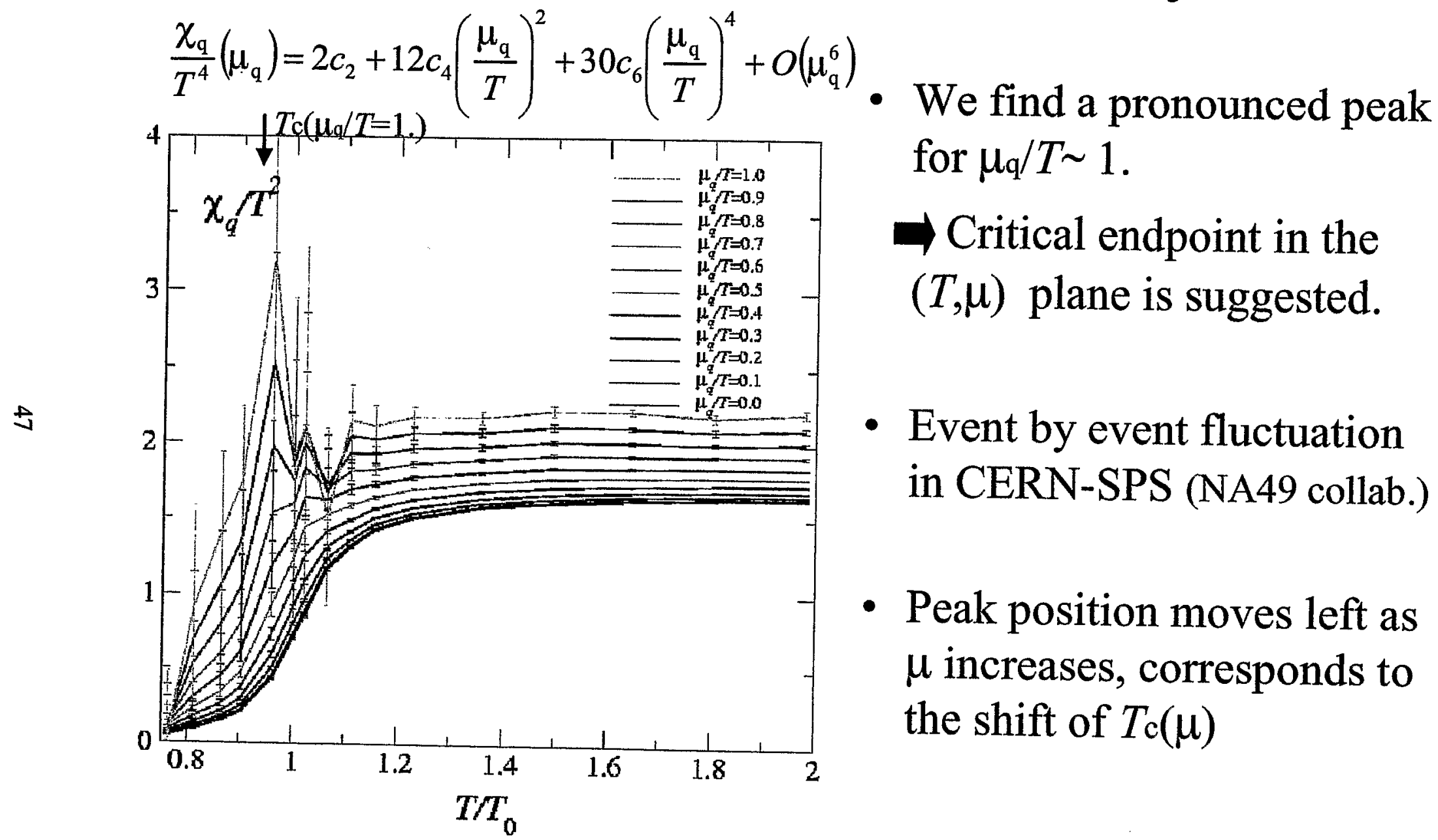




\section{Summary}

- Derivatives of pressure with respect to $\mu_{\mathrm{q}}$ up to $6^{\text {th }}$ order are computed.

- The convergence radius of Taylor expansion in $\mu \mathrm{q} / T$ seems to be large at high $T$ and $O(1)$ near $T_{\mathrm{c}}$.

- Quark number density fluctuation is discussed. $\chi_{\mathrm{q}}$ : A pronounced peak appears for $\mu / T_{0} \sim 1.0$.

$\Rightarrow$ This suggests the critical endpoint in $(T, \mu)$ plane.

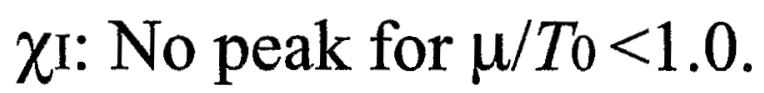

- Further studies at small quark and small lattice spacing are important.

- Study of strangeness is interesting. 


\title{
QCD at zero baryon density
}

\author{
Philippe de Forcrand (ETH \& CERN) \\ and \\ Slavo Kratochvila (ETH)
}

- Paradox:

Polyakov loop is $\neq 0$ in the $\mu=0$ grand-canonical ensemble. It is $=0$ in the zero-density canonical ensemble.

Are the two ensembles equivalent in the thermodynamic limit?

- We simulate the zero-density ensemble by integrating over an imaginary chemical potential.

No sign problem, negligible overhead.

- We demonstrate that the free energy minimum is the same in the two ensembles.

- By-product: the pressure as a function of $\mu$ and $T$.

- The non-zero Polyakov loop stems from sectors with fractional baryon number (non-zero triality), whose canonical partition function is zero. 


\section{The paradox}

chem. pot. $\mu$ conjugate to quark nb. $Q=\int d^{3} x \bar{\psi}(x) \gamma_{0} \psi(x)$

- Fix $\mu$ : grand-canonical ensemble $Z_{G C}$

- Fix $Q$ : canonical ensemble $Z_{C}$

equivalent in the thermodynamic limit. In particular,

$$
\mu=0 \Longleftrightarrow Q=0
$$

BUT Polyakov loop expectation value

$\mu=0:\langle P o l\rangle_{G C} \neq 0 \quad$ versus $\quad Q=0:\langle P o b l\rangle_{C}=0$

true for all volumes $V$ and temperatures $T$

- Equivalence of the two ensembles?

Oleszczuk \& Polonyi, Faber \& Borisenko, Azcoiti \& Galante,

".. "non-zero trallty" sectors?

- Order parameter confinement/deconfinement?

DeTar \& McLerran, Meyer-Ortmanns, Weiss, Fukushima, ...

Project: $(i)$ clarify and resolve paradox

(ii) numerical results for $Q=0$ 


\section{Connecting GC and $C$ ensembles}

Canonical $\rightarrow$ fix quark number $N \equiv \int d^{3} x \bar{\psi}(x) \gamma_{0} \psi(x)$ to $Q:$

$Z_{C}(Q)=\int[D U][d \bar{\psi}][d \psi] e^{-S_{g}[U]-S_{F}[U, \bar{\psi}, \psi]} \delta(N-Q)$

Fourier representation of $\delta$-function:

$=\frac{1}{2 \pi} \int_{0}^{2 \pi} d \bar{\mu}_{I} e^{-i Q \bar{\mu}_{I}} \times \int[D U][d \bar{\psi}][d \psi] e^{-S_{g}[U]-S_{F}[U, \bar{\psi}, \psi]+i \bar{\mu}_{I} N}$

$i \mu_{I}=i \bar{\mu}_{I} T$ is an imaginary chemical potential, and

$Z_{C}(Q)=\frac{1}{2 \pi} \int_{0}^{2 \pi} d \bar{\mu}_{I} e^{-i Q \frac{\mu_{I}}{T}} Z_{G C}\left(i \mu_{I}\right)$

Symmetries of $Z_{G C}\left(i \mu_{I}\right)$ :

Roberge \& Weiss

- even: $Z_{G C}\left(-i \mu_{I}\right)=Z_{G C}\left(+i \mu_{I}\right)$

- periodic: $Z_{G C}\left(i\left(\mu_{I}+2 \pi \frac{T}{3}\right)\right)=Z_{G C}\left(i \mu_{I}\right)$

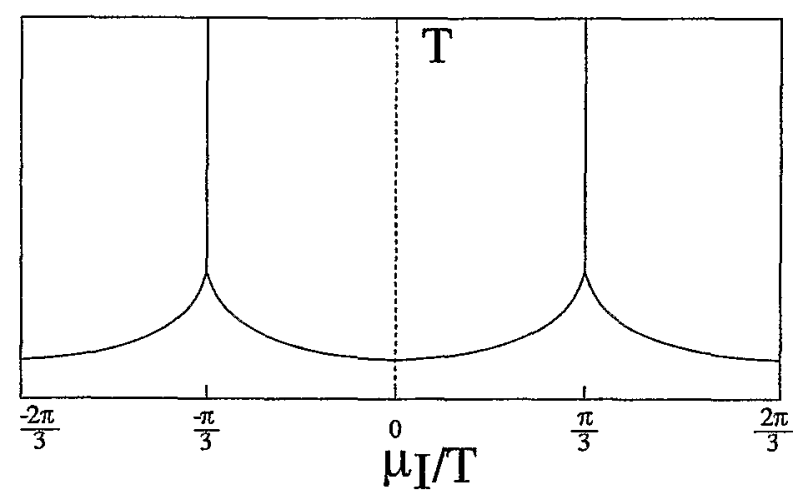

$\Rightarrow$ Fourier transform $Z_{C}(Q)$ vanishes unless $Q=0(\bmod 3)$

"Non-zero triality" sectors do not contribute. For $Q=3 B$ :

$$
Z_{C}(B)=\frac{1}{2 \pi} \int_{0}^{2 \pi} d \bar{\mu}_{I} e^{-i 3 B \frac{\mu_{J}}{T}} Z_{G C}\left(i \mu_{I}\right)
$$


Simulating $B=0$

How to sample

$Z_{C}(B=0)=\frac{1}{2 \pi} \int_{0}^{2 \pi} d \mu_{I} Z_{G C}\left(i \mu_{I}\right) ?$

with $Z_{G C}\left(i \mu_{I}\right)=\int[D U] e^{-S_{g}[U]} \operatorname{det}^{N_{f}} M\left(U ; i \mu_{I}\right)$

Make $\mu_{I}$ a dynamical degree of freedom, and alternate

- Standard Monte Carlo (HMC, R-algorithm, RHMC, ...)

to sample $Z_{G C}\left(i \mu_{I}\right)$, keeping $\mu_{I}$ fixed (no sign problem)

- noisy Metropolis update of $\mu_{I}$, keeping $\{U\}$ fixed

$\operatorname{Prob}\left(\mu_{I} \rightarrow \mu_{I}^{\prime}\right)=\min \left(1, \frac{\operatorname{det}^{N_{f}} M\left(U ; i \mu_{I}^{\prime}\right)}{\operatorname{det}^{N_{f}} M\left(U ; i \mu_{I}\right)}\right)$

Estimate ratio of determinants via

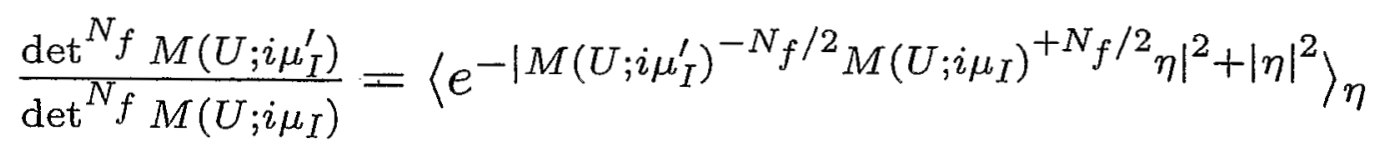

One Gaussian vector $\eta$ suffices.

Computational overhead totally negligible.

- Can supplement with " $Z_{3}$ moves" to help ergodicity:

$$
\mu_{I} \rightarrow \mu_{I} \pm \frac{2 \pi T}{3}, U_{4}(\vec{x}, t=0) \rightarrow U_{4}(\vec{x}, t=0) e^{\mp i \frac{2 \pi}{3}}, \forall \vec{x}
$$


Results: comparing $\mu=0$ and $B=0$

- Setup:

staggered quarks, $N_{f}=4$, am $=0.05, \mathrm{HMC}, 4^{3}, 6^{3}, 8^{3} \times 4$

- Distribution of $\operatorname{Tr}(P o l)$ in complex plane:

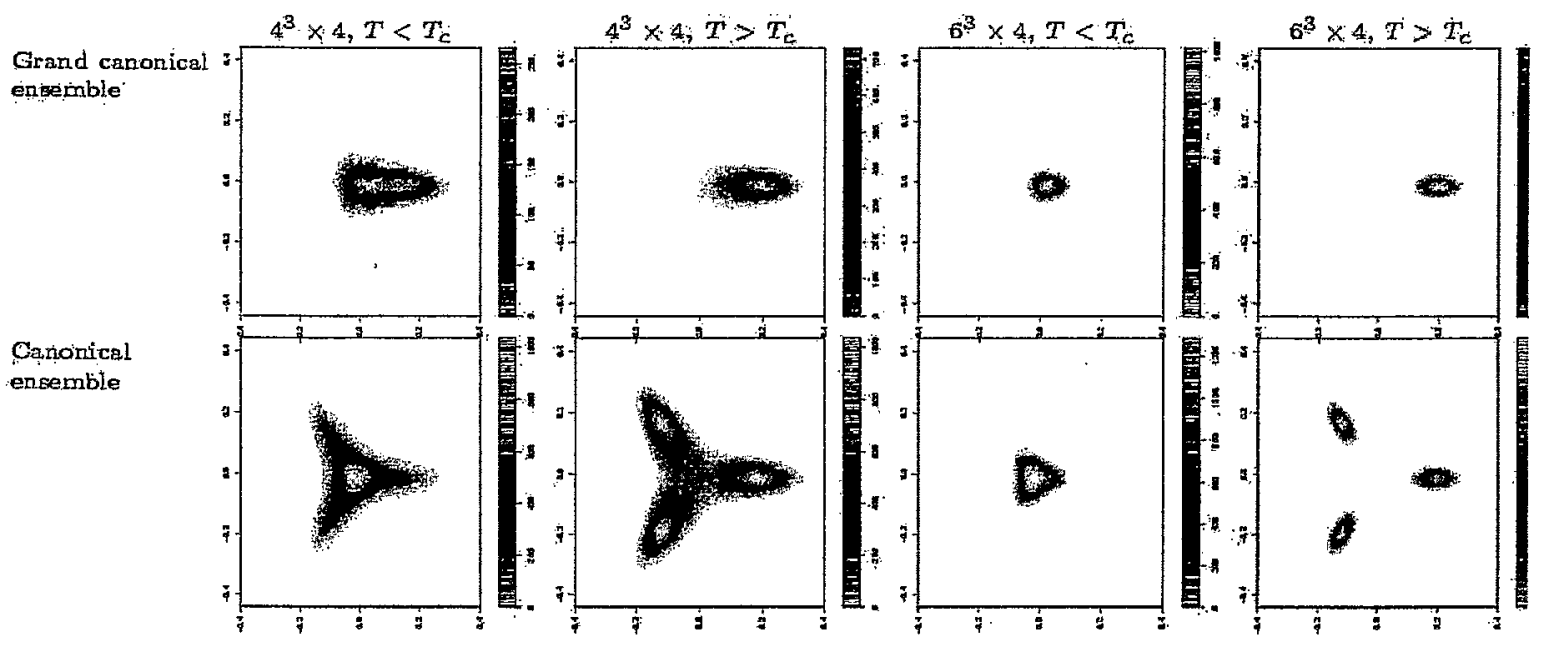

- canonical distribution is $Z_{3}$-symmetric

- canonical $\sim$ superposition of $Z_{3}$-rotated grand-canonical $\mu=$ 0 distributions

No big effect of $\mu_{I} \neq 0\left(\bmod \frac{2 \pi T}{3}\right)$ 
Free energy density versus $\mu_{I}$

$Z_{C}(B=0)=\frac{1}{2 \pi} \int_{0}^{2 \pi} d \mu_{I} Z_{G C}\left(i \mu_{I}\right)$

$\Rightarrow$ canonical simulation gives $F\left(\mu_{I}\right)=-\log Z_{G C}\left(\mu_{I}\right)$ for free, from $\mu_{I}$ histogram
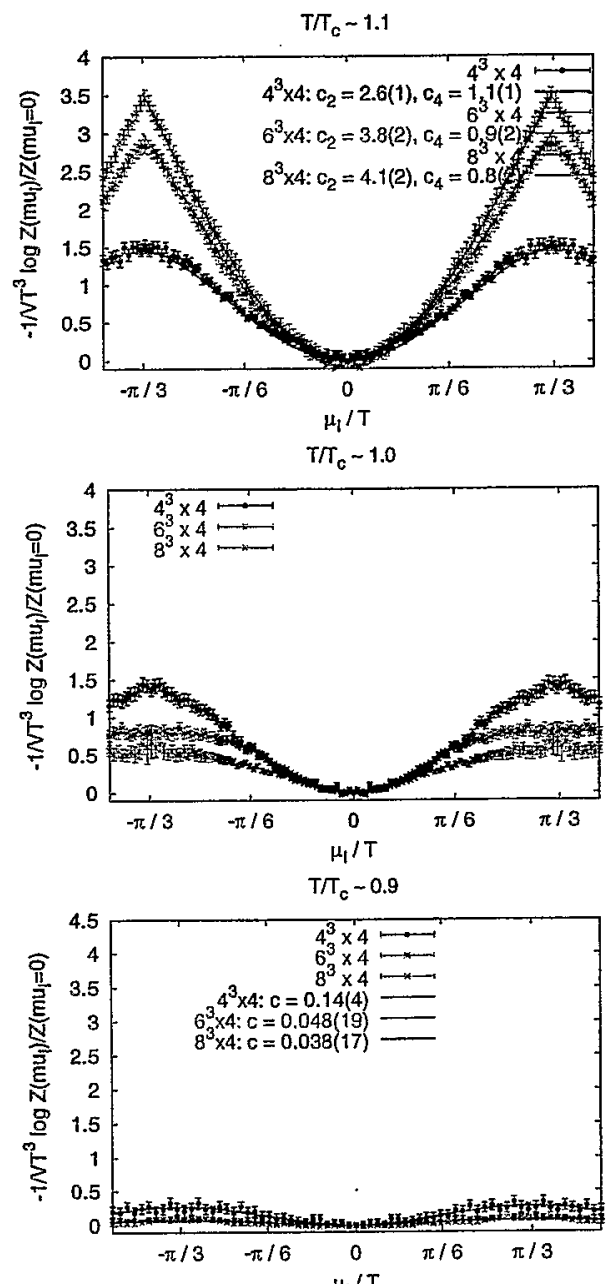

- Free energy density minimum at $\mu_{I}=0$ for all temperatures $\Longrightarrow \delta\left(\mu_{I}=0\right)$ in thermodynamic limit, i.e.

$$
B=0 \text { equivalent to } \mu=0
$$

- $\frac{F}{L^{3} T^{3}}=-\frac{p}{T^{4}} \rightarrow$ pressure vs $\mu_{I} / T$

Fit by truncated Taylor series in $\left(\frac{\mu_{I}}{T}\right)^{2}$ and analytically continue 


\title{
Potentials and Free Energies of Static Quarks
}

\author{
Owe Philipsen (University of Sussex)
}

- Introduction

- Gauge variant wave functions with gauge invariant energies

- Definitions of Static Potential

- Free energies

- Problems 
Why is it interesting?

\section{QGP and high density physics}

$\Rightarrow$ parton dynamics! $\Rightarrow$ colored intermediate states

Debye screening

Similarly: static charges in octet configuration

$T=0$ physics:

$J / \psi$ photoproduction in octet channel

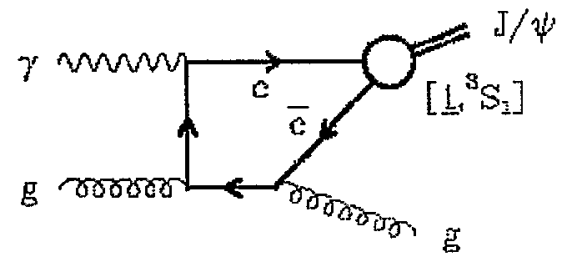

(a)

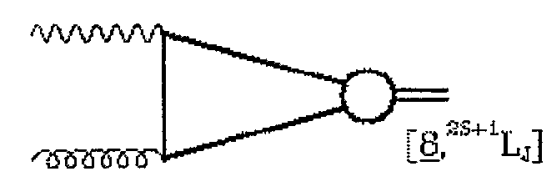

(b)

$\Rightarrow$ need non-pert. description of color dynamics 


\section{Strong coupling:}

gauge inv. ops. $\mathcal{O}$, e.g. plaquette

$$
\hat{H}_{0} O[U]=E_{O} O[U]
$$

strings: gauge variant states with gauge invariant eigenvalue

$$
\hat{H}_{0} U_{\alpha \beta}(\mathbf{x}, \mathbf{y})=E_{l} \dot{U}_{\alpha \beta}(\mathbf{x}, \mathbf{y})
$$

Multiply from right with $U^{\dagger}\left(\mathrm{x}_{0}, \mathrm{y}\right) T^{a} U\left(\mathrm{x}_{0}, \mathrm{y}\right)$ :

$$
\hat{H}_{0}\left[U\left(\mathbf{x}, \mathbf{x}_{\mathbf{0}}\right) T^{a} U\left(\mathbf{x}_{\mathbf{0}}, \mathbf{y}\right)\right]_{\alpha \beta}=E_{l}\left[U\left(\mathbf{x}, \mathbf{x}_{\mathbf{0}}\right) T^{a} U\left(\mathbf{x}_{\mathbf{0}}, \mathbf{y}\right)\right]_{\alpha \beta}
$$

With explicit source fields: $\bar{\psi}(\mathbf{x}) U(\mathbf{x}, \mathbf{y}) \psi(\mathbf{y})$ g.i.

not octet state, only its correlator

\section{Euclidean, any coupling:}

$$
\begin{aligned}
\left\langle\mathcal{O}^{\dagger}(t) \mathcal{O}(0)\right\rangle & =Z^{-1} \hat{\operatorname{Tr}}\left\{\hat{T}^{N_{t}-t} \hat{\mathcal{O}}^{\dagger}(0) \hat{T}^{t} \hat{\mathcal{O}}(0)\right\} \\
\langle W(r, t)\rangle & =Z^{-1} \hat{\operatorname{Tr}}\left\{\hat{T}^{N_{t}-t} \hat{U}_{\alpha \beta}(\mathbf{x}, \mathbf{y}) \hat{T}_{0}^{t} \hat{U}_{\alpha \beta}^{\dagger}(\mathrm{x}, \mathrm{y})\right\} \\
\Rightarrow \lim _{N_{t} \rightarrow \infty}\langle\ldots\rangle & =\sum_{n}\left|\left\langle 0\left|\hat{\mathcal{O}}(0) / \hat{U}_{\alpha \beta}(\mathbf{x}, \mathbf{y})\right| n\right\rangle\right|^{2} \mathrm{e}^{-\left(E_{n}-E_{0}\right) t}
\end{aligned}
$$


Nadkarni:

$q \bar{q}$ decomposition: $\bar{N} \otimes N=1+\left(N^{2}-1\right)$

Polyakov loop correlation: (potential at finite $T, \beta=1 / T$ )

$$
C_{\bar{q} q}(|\mathbf{x}|)=\left\langle L^{\dagger}(\mathbf{x}) L(0)\right\rangle=\mathrm{e}^{-\beta V_{1}} P_{1}+\mathrm{e}^{-\beta V_{a}} P_{a} .
$$

$P_{1, a}$ projection ops. on rep. matrices

$$
\begin{aligned}
\mathrm{e}^{-\beta V_{a v}} & =\frac{1}{N^{2}}\left\langle\operatorname{Tr} L^{\dagger}(\mathbf{x}) \operatorname{Tr} L(0)\right\rangle=\frac{1}{N^{2}} \mathrm{e}^{-\beta V_{1}}+\frac{N^{2}-1}{N^{2}} \mathrm{e}^{-\beta V_{a}} \\
\mathrm{e}^{-\beta V_{1}} & =\frac{1}{N}\left\langle\operatorname{Tr} L^{\dagger}(\mathbf{x}) L(0)\right\rangle
\end{aligned}
$$

gauge trafo: $L^{g}(\mathbf{x})=g(\mathbf{x}) L(\mathbf{x}) g^{-1}(\mathbf{x})$

$\Rightarrow$ only $V_{a v}$ gauge inv.

Hibert space formulation:

Static quark propagation $\psi_{\alpha}\left(\mathrm{x}, N_{t}\right)=L(\mathrm{x})_{\alpha \beta} \psi_{\beta}(\mathrm{x}, 0)$

$$
\begin{aligned}
\mathrm{e}^{-\beta V_{a v}} & =\hat{\operatorname{Tr}}\left\{\hat{\psi}_{\beta}^{c}(\mathbf{y}) \hat{\psi}_{\alpha}(\mathbf{x}) \hat{T}^{N_{t}} \hat{\psi}_{\alpha}^{\dagger}(\mathbf{x}) \hat{\psi}_{\beta}^{c \dagger}(\mathbf{y})\right\} \\
\mathrm{e}^{-\beta V_{1}} & =\hat{\operatorname{Tr}}\left\{\hat{\psi}_{\alpha}^{c}(\mathbf{y}) \hat{\psi}_{\alpha}(\mathbf{x}) \hat{T}^{N_{t}} \hat{\psi}_{\beta}^{\dagger}(\mathbf{x}) \hat{\psi}_{\beta}^{c \dagger}(\mathbf{y})\right\}
\end{aligned}
$$

second line singlet only for $\mathrm{x}=\mathrm{y}$ 
Dressed sources:

op.

Consider functional $\Omega\left[U_{i}\right]$ in every t-slice, with

$$
\Omega^{g}(\mathbf{x}, t)=g(x) \Omega(\mathbf{x}, t) h(t)
$$

(e.g. gauge fixing function of Coulomb gauge)

$$
\begin{aligned}
& \psi^{\Omega}(\mathbf{x})= \Omega^{\dagger}(\mathbf{x}) \psi(\mathbf{x}) \\
& \Rightarrow\left(\bar{\psi}^{\Omega}(\mathbf{x}) \psi^{\Omega}(\mathbf{y})\right)^{g}=\bar{\psi}^{\Omega}(\mathbf{x}) \psi^{\Omega}(\mathbf{y}) \\
&\left(\bar{\psi}^{\Omega}(\mathbf{x}) T^{a} \psi^{\Omega}(\mathbf{y})\right)^{g}=\left(\psi^{\Omega \dagger}(\mathbf{x}) h^{\dagger} T^{a} h \psi^{\Omega}(\mathbf{y})\right)
\end{aligned}
$$

correct trafo for all $\mathrm{x}, \mathrm{y}$ !

$\Rightarrow$ Dressed PL: $\tilde{L}(\mathrm{x})=\Omega^{\dagger}(\mathrm{x}) L(\mathrm{x}) \Omega(\mathrm{x})$

$\operatorname{Tr} \tilde{L}=\operatorname{Tr} L$, average potential the same

But now $\left(L^{\Omega}\right)^{g}=h L^{\Omega} h^{-1}$

$\Rightarrow\left\langle\operatorname{Tr} \tilde{L}^{\dagger}(\mathbf{x}) \tilde{L}(0)\right\rangle$ singlet, average correlators separately invariant!

$T=0$ :

$$
\begin{gathered}
\left\langle\operatorname{Tr} \tilde{L}^{\dagger}(\mathbf{x}) \tilde{L}(\mathbf{y})\right\rangle= \\
Z^{-1} \hat{\operatorname{Tr}}\left\{\hat{T}^{N_{t}-t}\left(\hat{\Omega}(\mathbf{x}) \hat{\Omega}^{\dagger}(\mathbf{y})\right)_{\alpha \beta} \hat{T}_{0}^{t}\left(\hat{\Omega}^{\dagger}(\mathbf{y}) \hat{\Omega}(\mathbf{x})\right)_{\beta \alpha}\right\}
\end{gathered}
$$


Numerical results in $2+1 \mathrm{~d} \mathrm{SU}(2)$

Wilson loop vs. dressed Wilson line

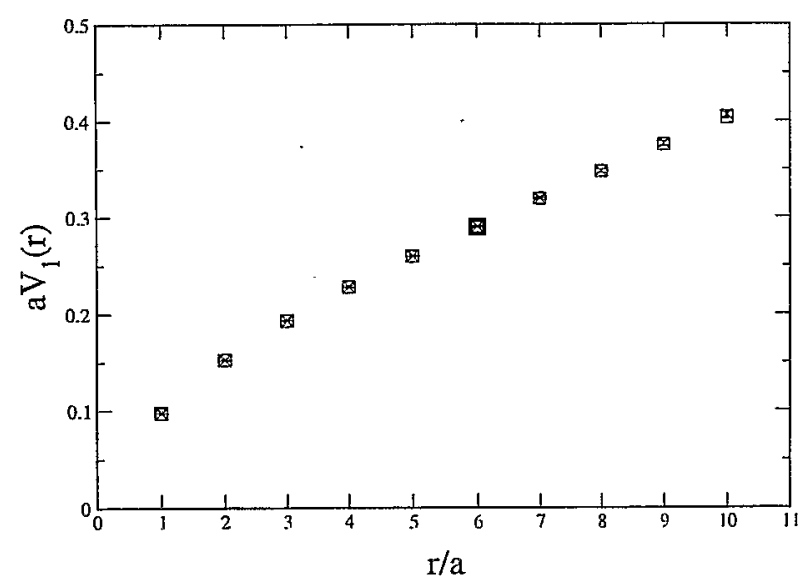

$T \neq 0: \quad$ Def: $\quad \mathrm{e}^{-V_{i}(T) / T}:=\sum_{n} \mathrm{e}^{-V_{i}^{n} / T} \quad$ free energies
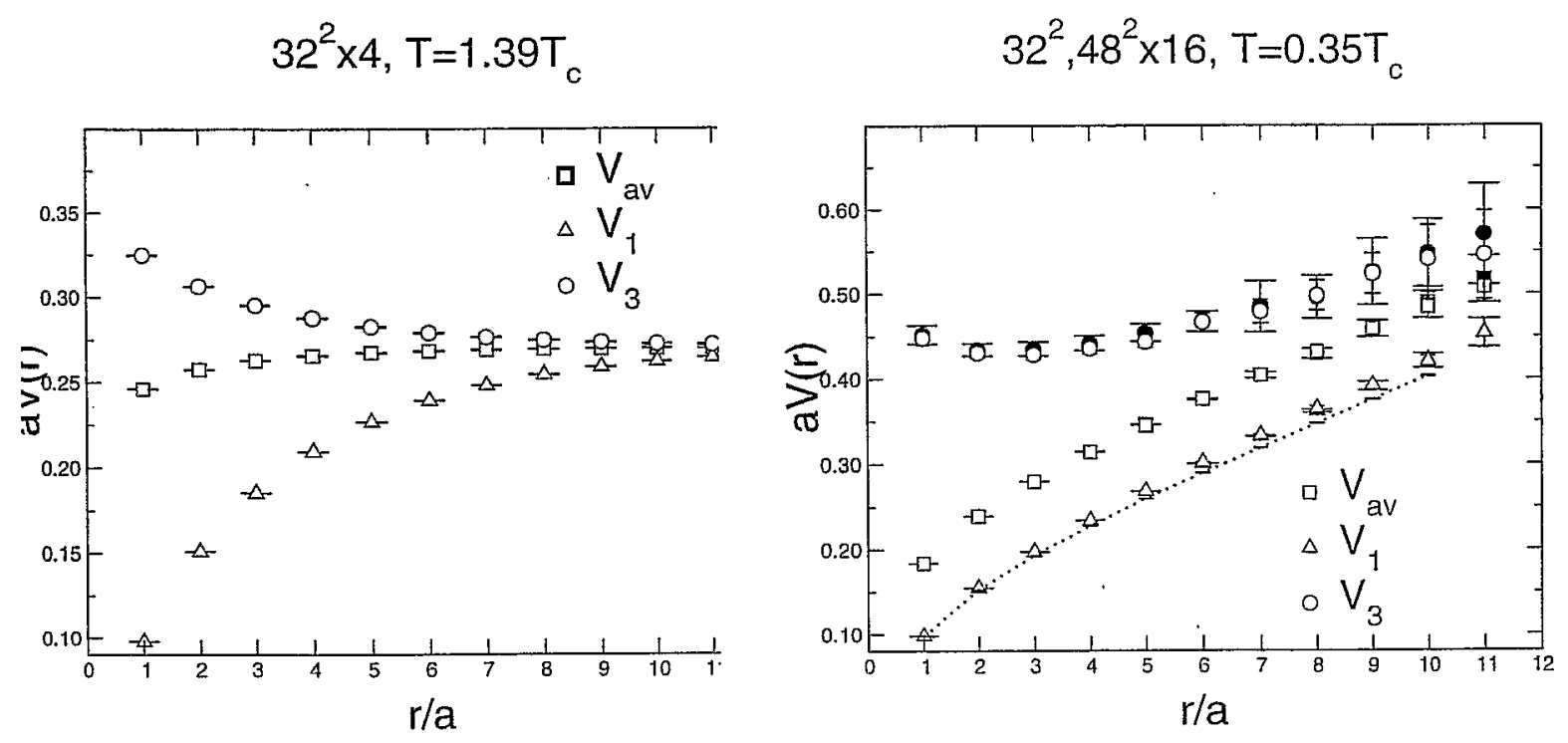


\title{
New Developments in the field of Polyakov Loop Correlation Functions
}

\author{
O. Kaczmarek, F. Karsch, P. Petreczky and F. Zantow ${ }^{1}$
}

\begin{abstract}
and further notes

One of the basic approaches in the discussion of thermal properties of heavy quark bound states is based on the analysis of the non-relativistic Schroedinger-Eq. using potentials calculated in, or motivated through, lattice calculations. So far, however, lattice calculations at finite temperatures provided the color averaged free energies rather than the color singlet energies which could define the finite temperature potential. Of even greater importance than the free energy for the analysis of the confinement forces is the potential energy at finite temperature, however, its calculation at finite temperature is still missing. Moreover, the role of any entropy in the free energy has been ignored so far in potential models using free energies.
\end{abstract}

This Talk is organized as follows: We mainly analyze the singlet heavy quark antiquark free energies calculated from the Polyakov loop correlation function in $S U(3)$ gauge theory (quenched QCD) on the lattice at finite temperature. We start our analysis by discussing the thermal properties of the running coupling $\left(\alpha=g^{2} / 4 \pi\right)$ at finite temperature defined on the basis of leading order perturbation theory for singlet free energies. While at large distances the coupling scales with the temperature, i.e. $\alpha=\alpha(T)$, at small distances the coupling recovers vacuum physics, i.e. $\alpha=\alpha(1 / r)$.

We argue that the short distance, zero temperature dominated properties of the quark antiquark free energies can be used to define the renormalized free energies and the renormalized Polyakov loop. The renormalized Polyakov loop does show no significant dependence on the temporal lattice extent $\left(N_{\tau}\right)$, although lattices were used with $N_{\tau}=4,8$ and $N_{\tau}=16$. This shows that the renormalized Polyakov loop is well-behaved in the continuum limit.

Finally we present first lattice results for the quark antiquark potential energy $(\Delta E)$ at finite temperature which we extract from the (renormalized) free energies $(\Delta F)$ by applying thermal relations, i.e. $\Delta E=-T^{2} d(\Delta F / T) / d T$. In fact, the potential energies show a surprisingly different behavior from the behavior of the free energies as the entropy turns out to be quite important in the free energy.

${ }^{1}$ Talk given by FZ. For Refs. and further questions contact zantow@physil.uni-bielefeld.de 


\section{Color singlet free energies}

- Short and large distances -

From one-gluon exchange:

$\delta \mathbf{F}_{1}(\mathbf{r}, \mathbf{T}) \simeq\left\{\begin{aligned}-\frac{\mathbf{g}^{2}(\mathbf{T})}{\mathbf{3} \pi \mathbf{r}} \mathbf{e}^{-\mu(\mathbf{T}) \mathbf{r}} & : \text { for } \mathbf{r} \mathbf{T} \gg \mathbf{1} \\ -\frac{\mathbf{g}^{2}(\mathbf{r})}{\mathbf{3} \pi \mathbf{r}} & : \text { for } \mathbf{r} \Lambda_{\mathrm{QCD}} \ll \mathbf{1}\end{aligned}\right.$

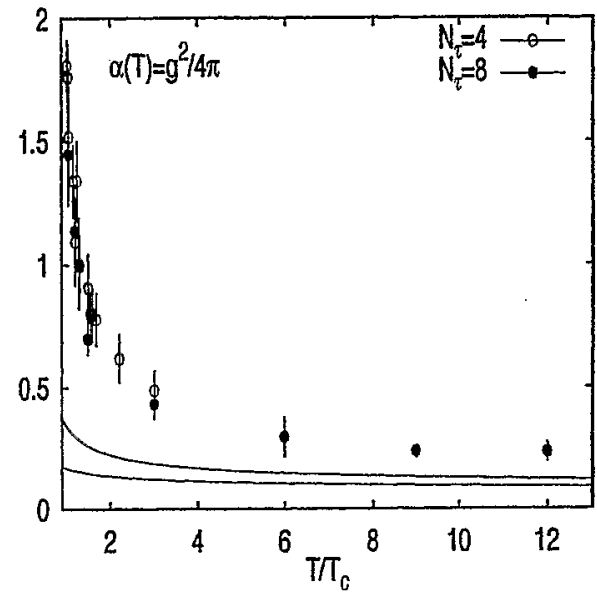

Define:

$\mathbf{S}_{1}(\mathbf{r}, \mathbf{T}) \equiv-\frac{\mathbf{3}}{\mathbf{4}} \mathbf{r} \mathbf{T} \times \frac{\delta \mathbf{F}_{1}(\mathbf{r}, \mathbf{T})}{\mathbf{T}}$

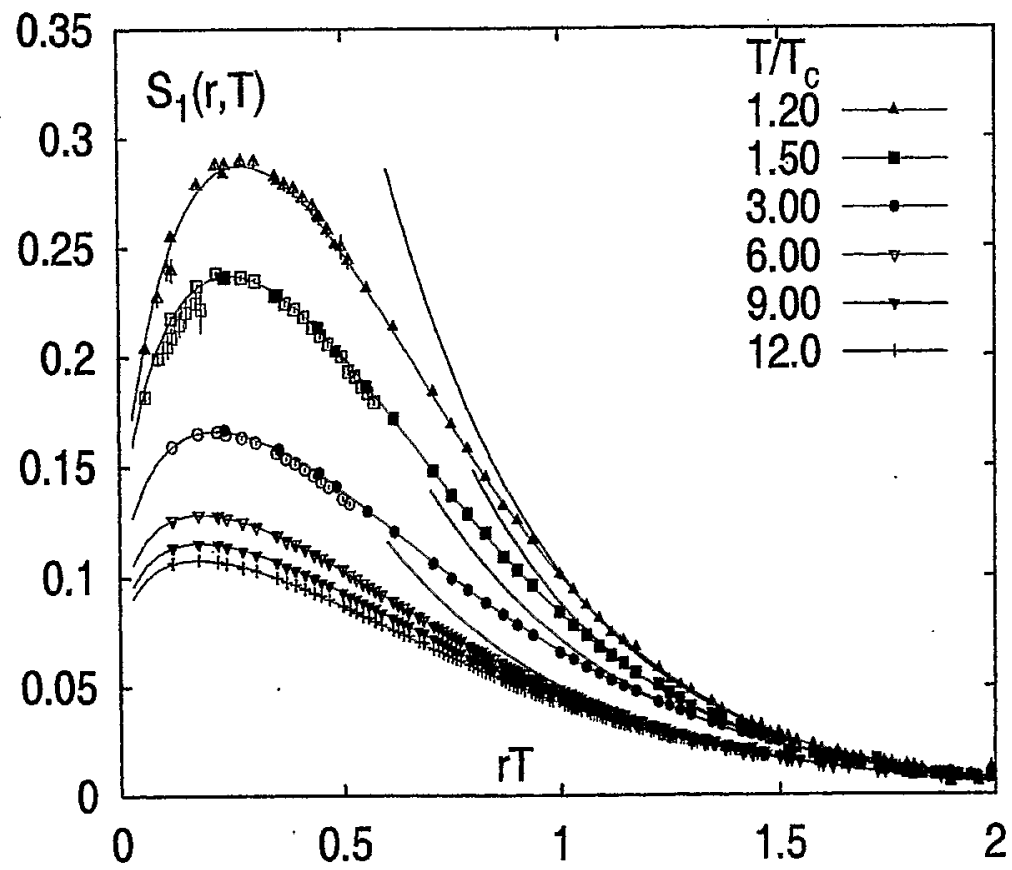

The $r$-dependence of the coupling becomes quite important:

$$
\mathrm{g}^{2}=\mathrm{g}^{2}(\mathrm{r}, \mathrm{T})
$$




\section{Lattice renormalization of free energies}

- Renormalization of the Polyakov Loop -

$$
\begin{aligned}
& \begin{array}{c}
\text { Divergences appear: } \\
\text { A. M. Polyakov (1980) } \\
\langle\operatorname{TrL}\rangle \simeq \mathrm{e}^{- \text {lin.div. } \times \mathrm{G}_{\mathrm{Ien}}} \\
\simeq \mathrm{e}^{-\mathrm{cN}_{\tau}} \times \mathrm{G}_{\mathrm{ren}}
\end{array} \\
& \text { Proof: } \\
& \text { I. Ya. Arefeva (1980); V. S. Dotsenko and S. N. Vergeles (1980); J. L. Gervais and A. Neveu (1980) } \\
& \text { How to remove the linear divergence on the lattice? }
\end{aligned}
$$

Concept of non-perturbative renormalization

- 1st step: $r \ll 1 / T, \quad \Delta F_{q \bar{q}}(r, T) \simeq V_{q \bar{q}}(r)$

- 2nd step: $\mathbf{r} \gg \mathbf{I} / \mathbf{T}, \quad|\langle\operatorname{Tr} \mathbf{L}\rangle|=\lim _{|\mathbf{r}| \rightarrow \infty}\left(\left\langle\operatorname{Tr} \mathbf{L}(\mathbf{0}) \operatorname{Tr} \mathbf{L}^{\dagger}(\mathbf{r})\right\rangle\right)^{\mathbf{1} / 2}$

(Definition!)

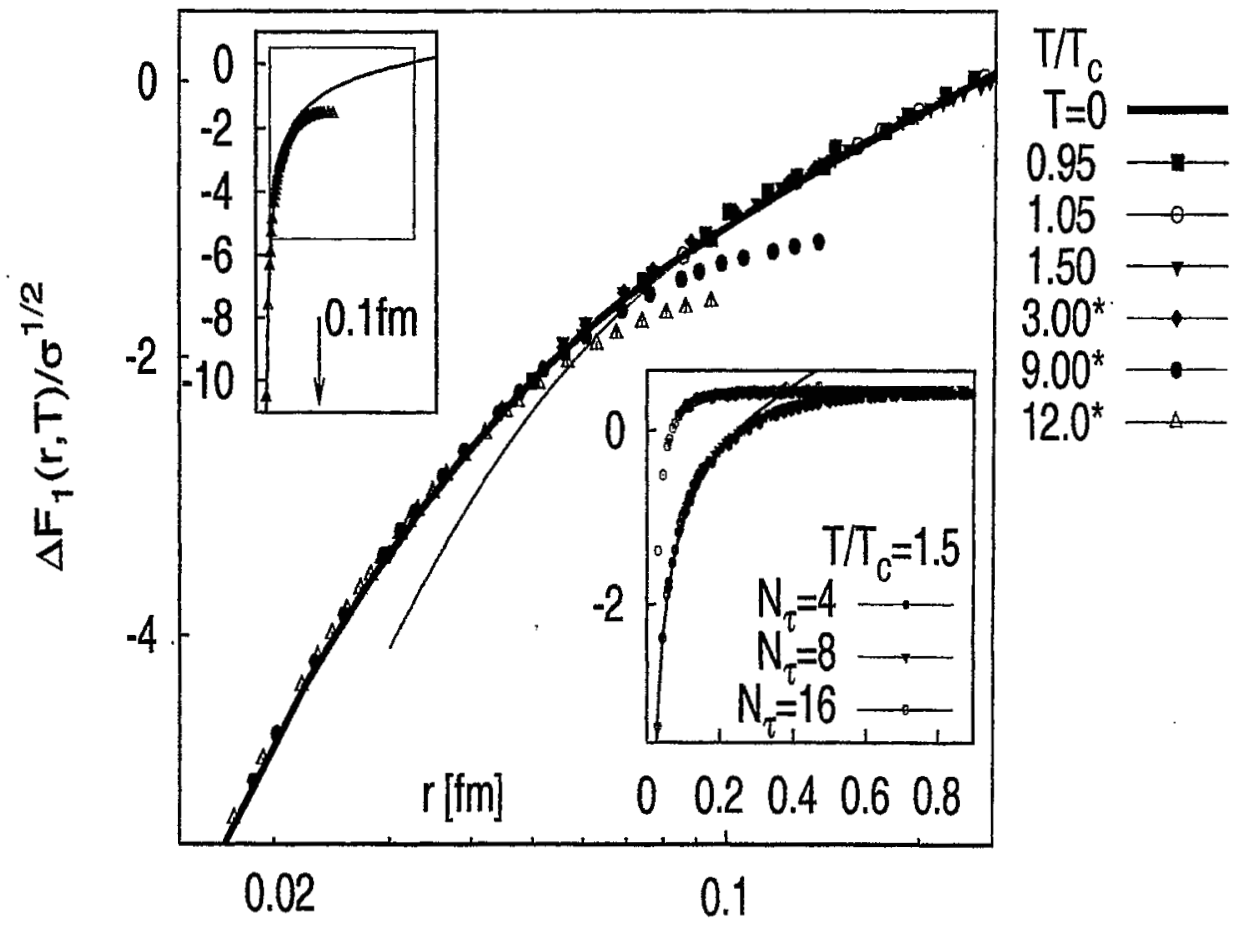




\section{The renormalized Polyakov loop \\ - here: SU(3) -}

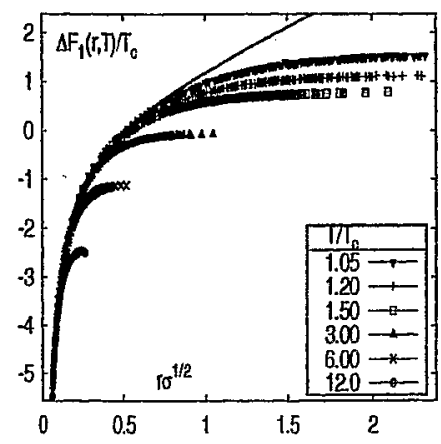

$$
\frac{\Delta \mathbf{F}_{\mathbf{q}}}{\mathbf{T}} \equiv \frac{1}{2} \lim _{\mathbf{r} \rightarrow \infty} \frac{\Delta \mathbf{F}_{\mathbf{q} \mathbf{q}}^{\mathbf{R}}(\mathbf{r}, \mathbf{T})}{\mathbf{T}} \text { fixed! }
$$

Renormalized Polyakov loop:

$$
\left|\left\langle\operatorname{Tr}^{\mathbf{R}}\right\rangle\right|=\exp \left(-\frac{\Delta \mathbf{F}_{\mathbf{q}}}{\mathbf{T}}\right)
$$

The lattice-renormalized Polyakov loop: Results from SU(3)

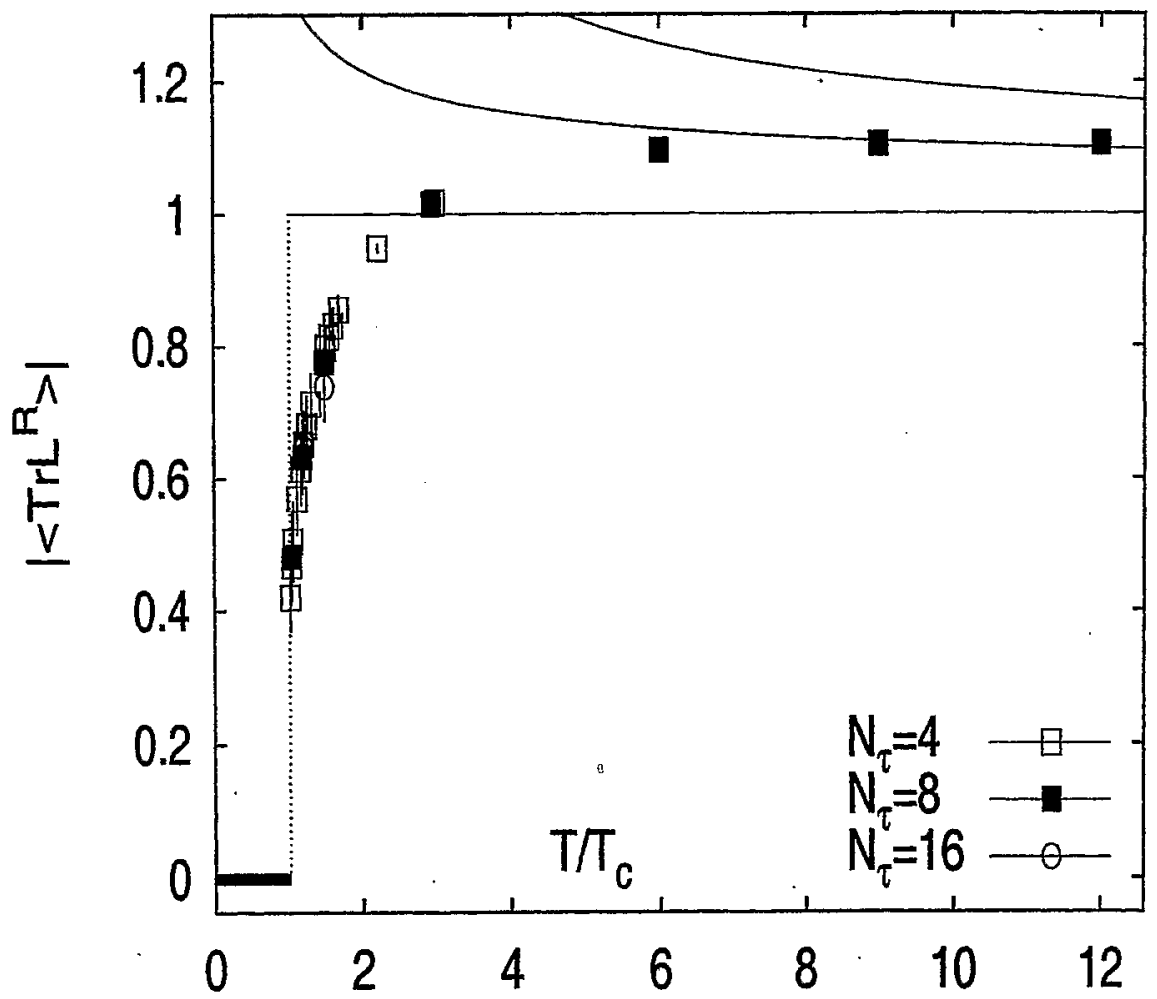

Continuum PT: E. Gava and R. Jengo (1981) 
Free energy vs. Potential energy

- Lattice results: $T>T_{c}$ -
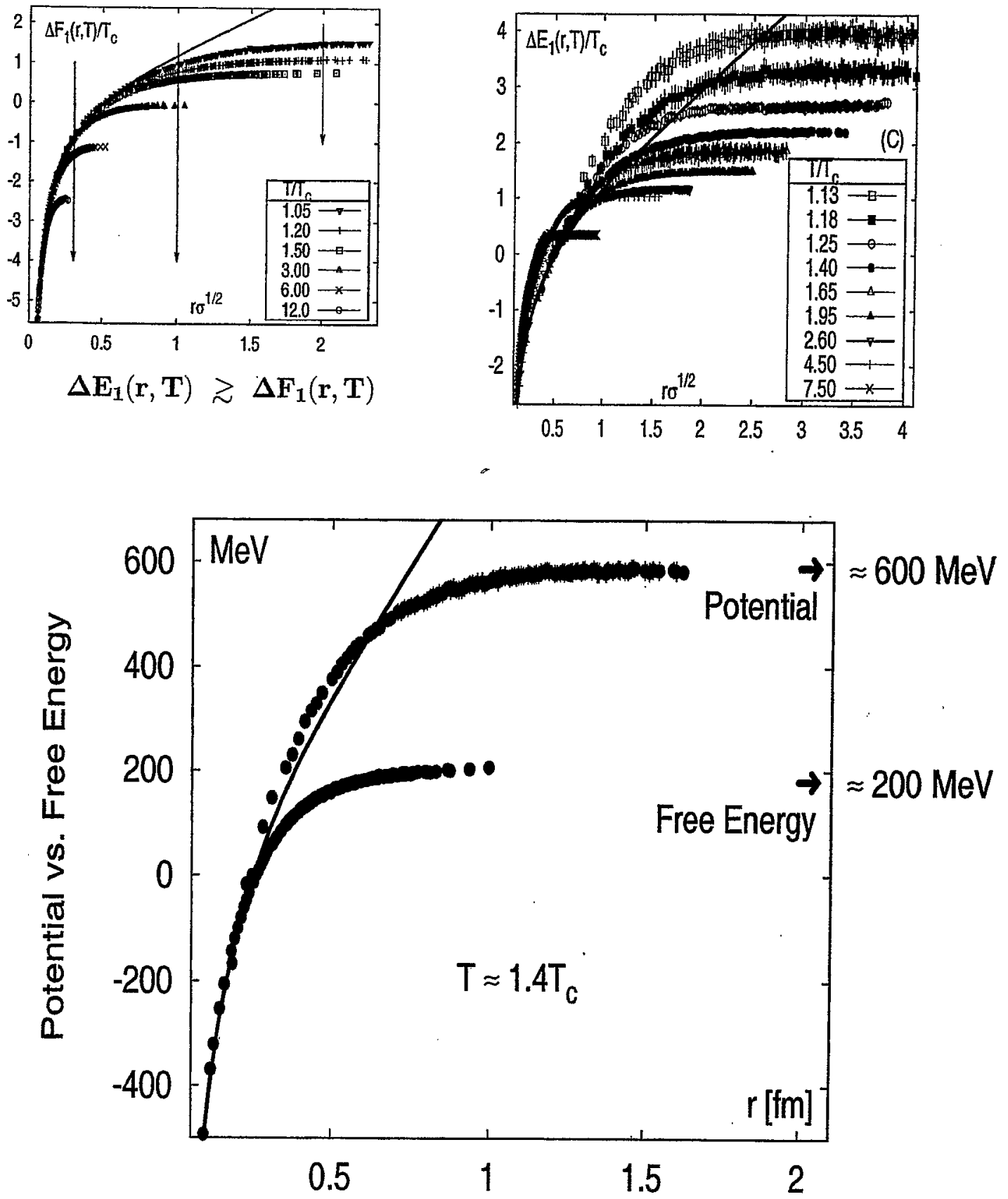

Consequences for $J / \Psi$-suppression? 
F. Zantow, at BNL, 02.07-02.13.04

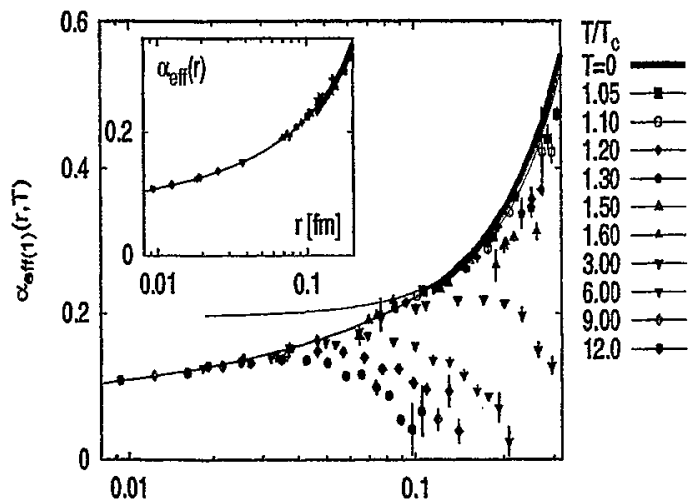

\section{Summary \\ - A strategy for future analysis? -}
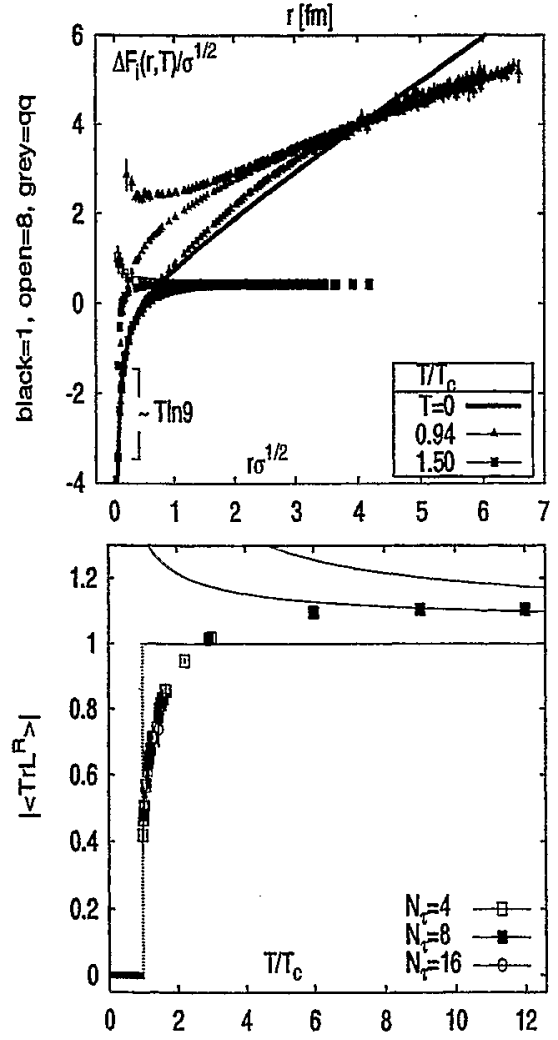

Analysis of $n$-point PLCs

- in $\mathrm{SU}(\mathrm{N})$

- in full QCD

- in full QCD and finite densities
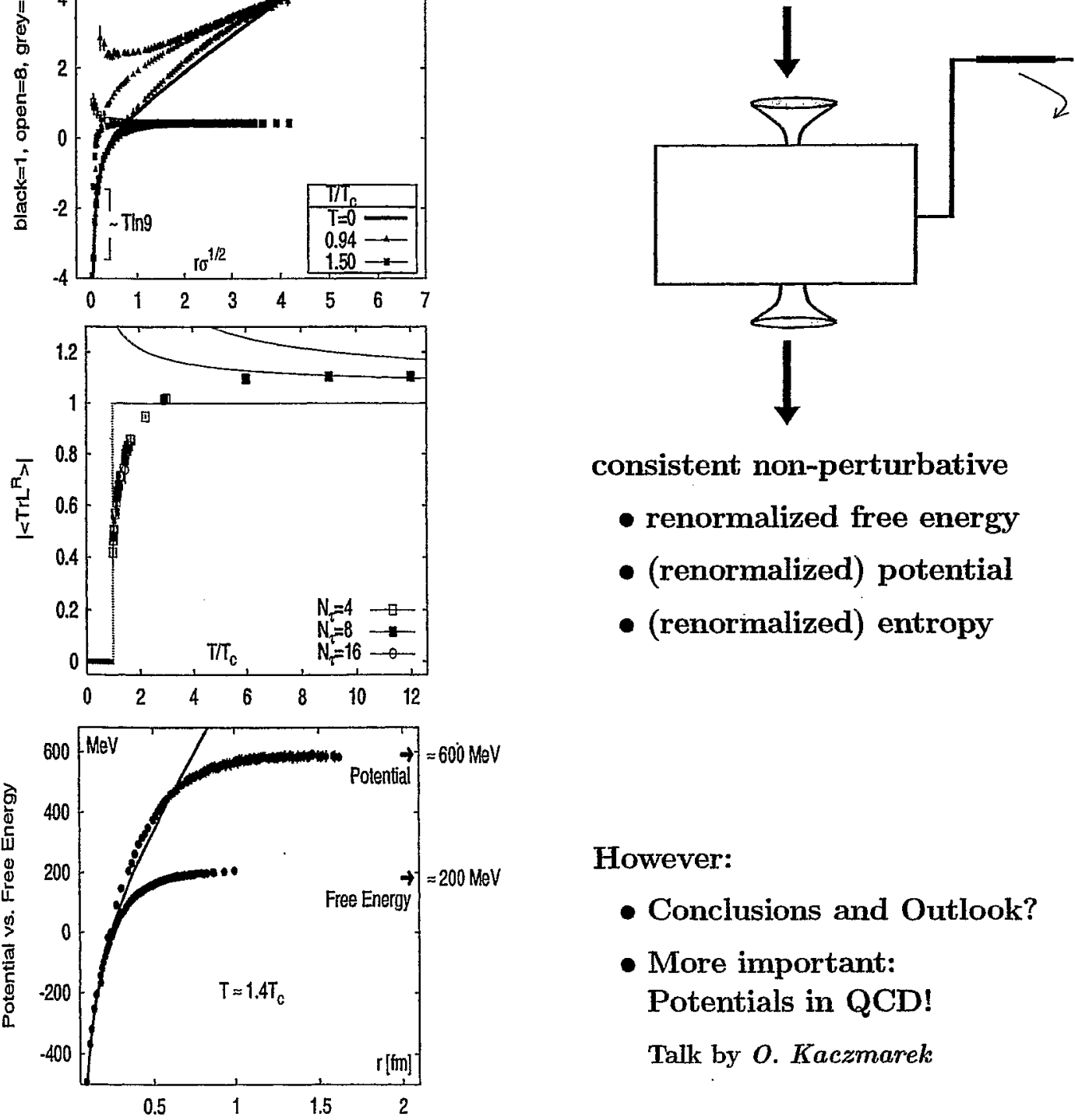

However:

- Conclusions and Outlook?

- More important:

Potentials in QCD!

Talk by $O$. Kaczmarek 


\title{
Heavy Quark free energies and the renormalized Polyakov in full QCD
}

\author{
O. Kaczmarek, S. Ejiri, F. Karsch, E. Laermann, F. Zantow \\ Fakultät für Physik, Universität Bielefeld
}

We will discuss first results on the heavy quark free energies in the different color channels in QCD with dynamical quarks. The basic concepts on the renormalization of the free energies and results for the quenched theory can be found in the contribution by F. Zantow. While in earlier studies of the heavy quark free energy in full QCD only the color averaged operators were analyzed, we have a quite detailed description of the different color channels in the quenched theory.

At very small distances, the dominant scale is set by the distance $r$ and the free energies are expected to be well described by the zero temperature potential, i.e. zero temperature perturbation theory at sufficiently small $r$. This is used to renormalize the free energies and therefore eliminating the divergent self-energy contributions by matching them at small $r$ to the $T=0$ potential.

In contrast to a linear rising potential in the quenched theory, in QCD with dynamical fermions the free energies below $T_{c}$ show a different behavior at large separations. Due to the possibility of pair creation the string between the two test quarks can break, leading to a constant free energy at large separations.

Above $T_{c}$, the free energies are exponentially screened at large distances $(r T \gg 1)$ due to the generation of a chromoelectric (Debye) mass. At very small separations, $r T \ll 1$, one gets into the perturbative regime, where the relevant scale is again set by the distance $r$ and no temperature effects are seen, even at high temperatures in the deconfined phase. In this region, the singlet free energy is well described by the zero temperature potential and the running coupling depends on the dominant scale, i.e. $g=g(r)$.

To see where the $T$-independent short distance behavior and the temperature dependent large distance behavior are separated, we have calculated the screening function and the effective running coupling. While at large distances temperature effects are visible, i.e. screening and a $T$-depending running coupling, $g(T)$, the short distance behavior is governed by zero temperature physics where the only scale is set by $r$ and the running coupling $g(r)$ depends only on this scale. The temperature effects set in at smaller distance with increasing temperature.

We define the renormalized Polyakov loop $L_{\text {ren }}$ by the large distance behavior of the heavy quark free energies. While $L_{r e n}$ is zero in the quenched theory below $T_{c}$, it is non-zero in full QCD for all temperatures. At temperatures above $T_{c}$ the behavior of the renormalized Polyakov loop is comparable to the quenched case. 


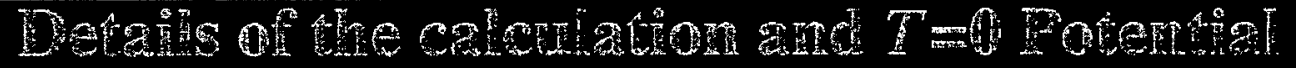

$N_{f}=2$ with $m / T=0.4\left(m_{\pi} / m_{\mathrm{\rho}} \approx 0.7\right.$ at $\left.T_{c}\right)$

Lattice size: $16^{3} \times 4, T_{c} \approx 170 \mathrm{MeV}$

(generated by the Bielefeld-Swansea collaboration)

Symanzik improved gauge action and p4-improved staggered fermion action

Physical scale is determined by string tension $T=0$ potential obtained from Wilson loops

[F. Karsch, E. Laermann and A. Peikert, Nucl. Phys . B 605 (2001) 579]

Coulomb gauge fixing [O. Philipsen, Phys. Lett. B535 (2002) 138]

$$
\begin{aligned}
-\ln \left(\left\langle\tilde{\operatorname{Tr}} L(\mathbf{x}) \tilde{\operatorname{Tr}} L^{\dagger}(\mathbf{y})\right\rangle\right) & =\frac{F_{\bar{q} q}(R, T)}{T} \\
-\left.\ln \left(\left\langle\tilde{\operatorname{Tr}} L(\mathbf{x}) L^{\dagger}(\mathbf{y})\right\rangle\right)\right|_{G F} & =\frac{F_{1}(R, T)}{T} \\
-\ln \left(\frac{9}{8}\left\langle\tilde{\operatorname{Tr}} L(\mathbf{x}) \tilde{\operatorname{Tr}} L^{\dagger}(\mathbf{y})\right\rangle-\left.\frac{1}{8}\left\langle\tilde{\operatorname{Tr}} L(\mathbf{x}) L^{\dagger}(\mathbf{y})\right\rangle\right|_{G F}\right) & =\frac{F_{8}(R, T)}{T}
\end{aligned}
$$




\section{Heavy quark free energy}
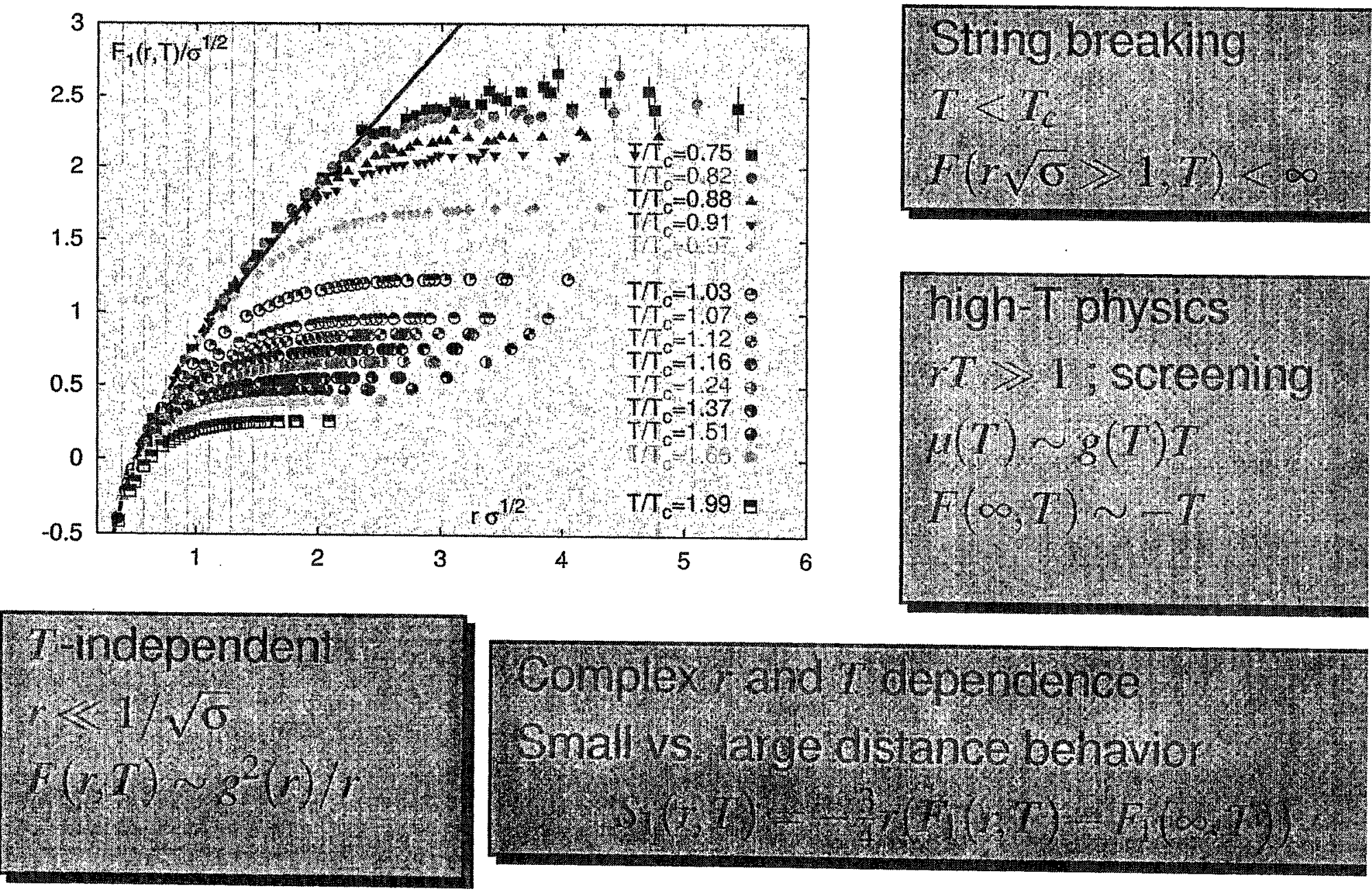


\section{Short vs. long distance behaviour}
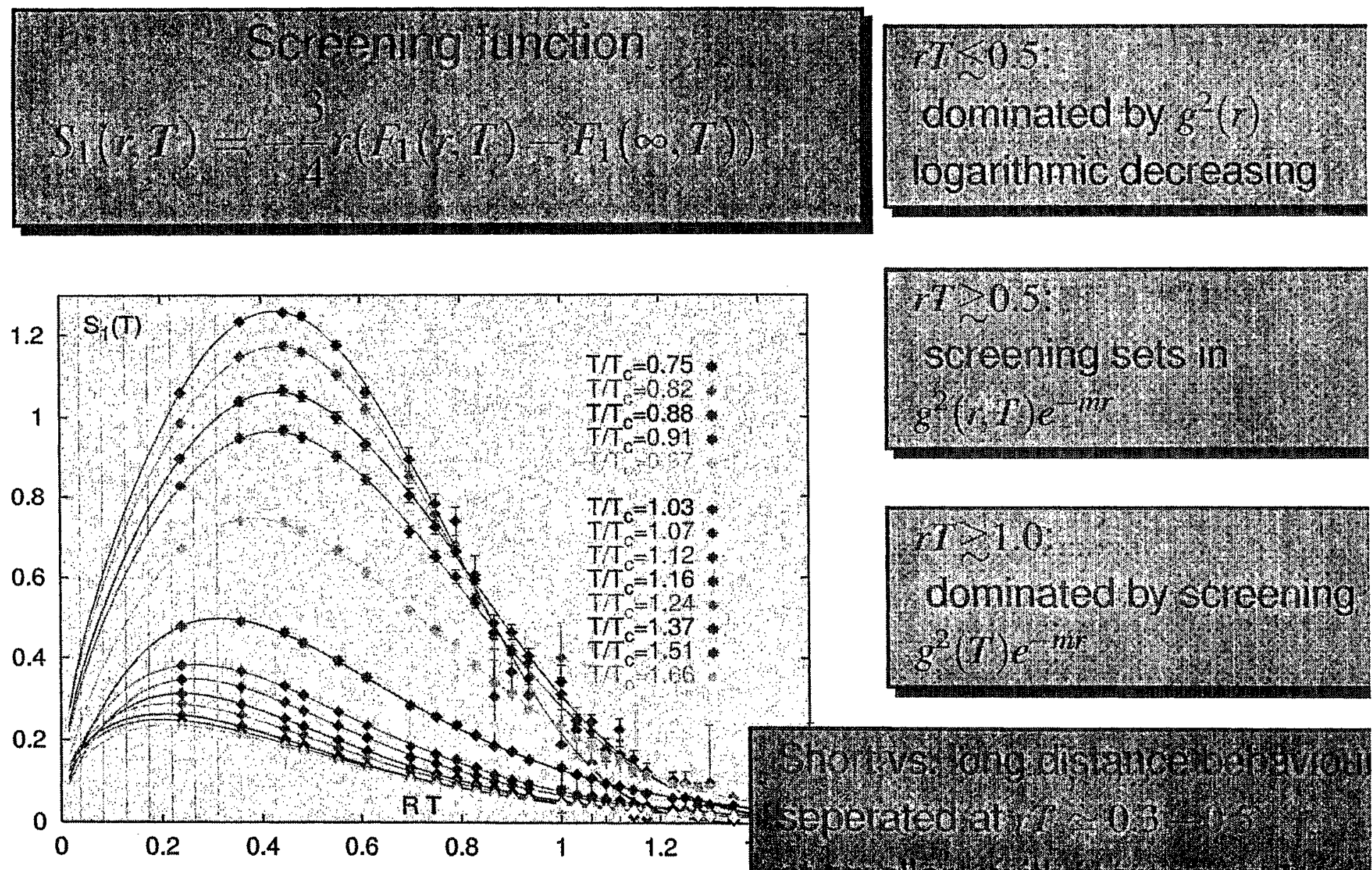

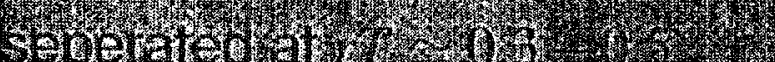

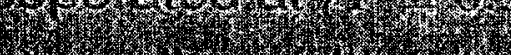

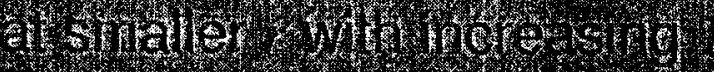




\section{Running coupling vs. Screening}

Effective running coupling:

$$
\alpha_{\mathrm{e} f f}(r, T)=\frac{3 r^{2}}{4} \frac{\mathrm{d} F_{1}(r, T)}{\mathrm{d} r}
$$

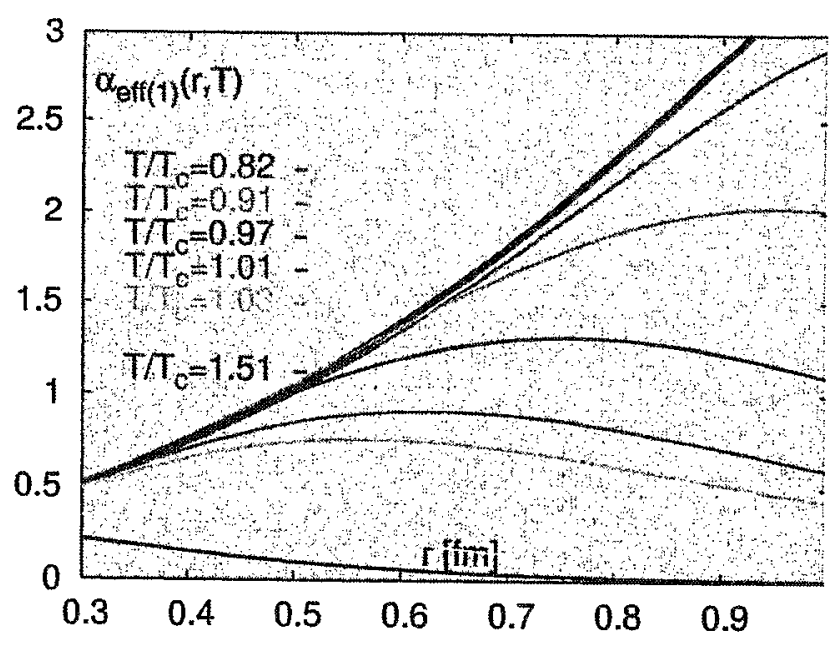

Screening dominates at $r T \gg 1$

Larger lattices needed to extract screening properties

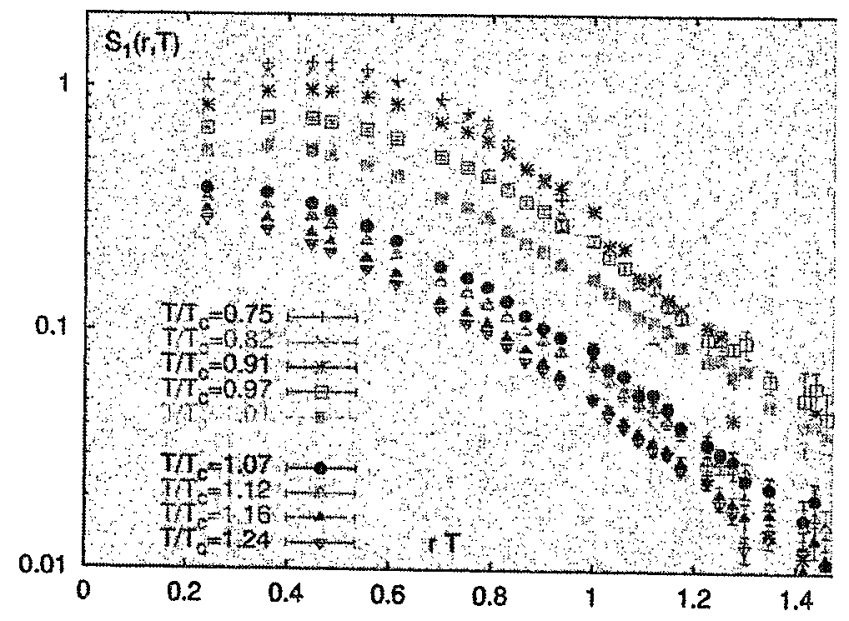




\section{Renormalized Polyakov loop}

$$
\begin{aligned}
& L_{\mathrm{ren}}=\exp \left(-\frac{F(r=\infty, T)}{2 T}\right) \\
& L_{\mathrm{ren}}=0 \text { for } T<T_{C} \text {. }
\end{aligned}
$$

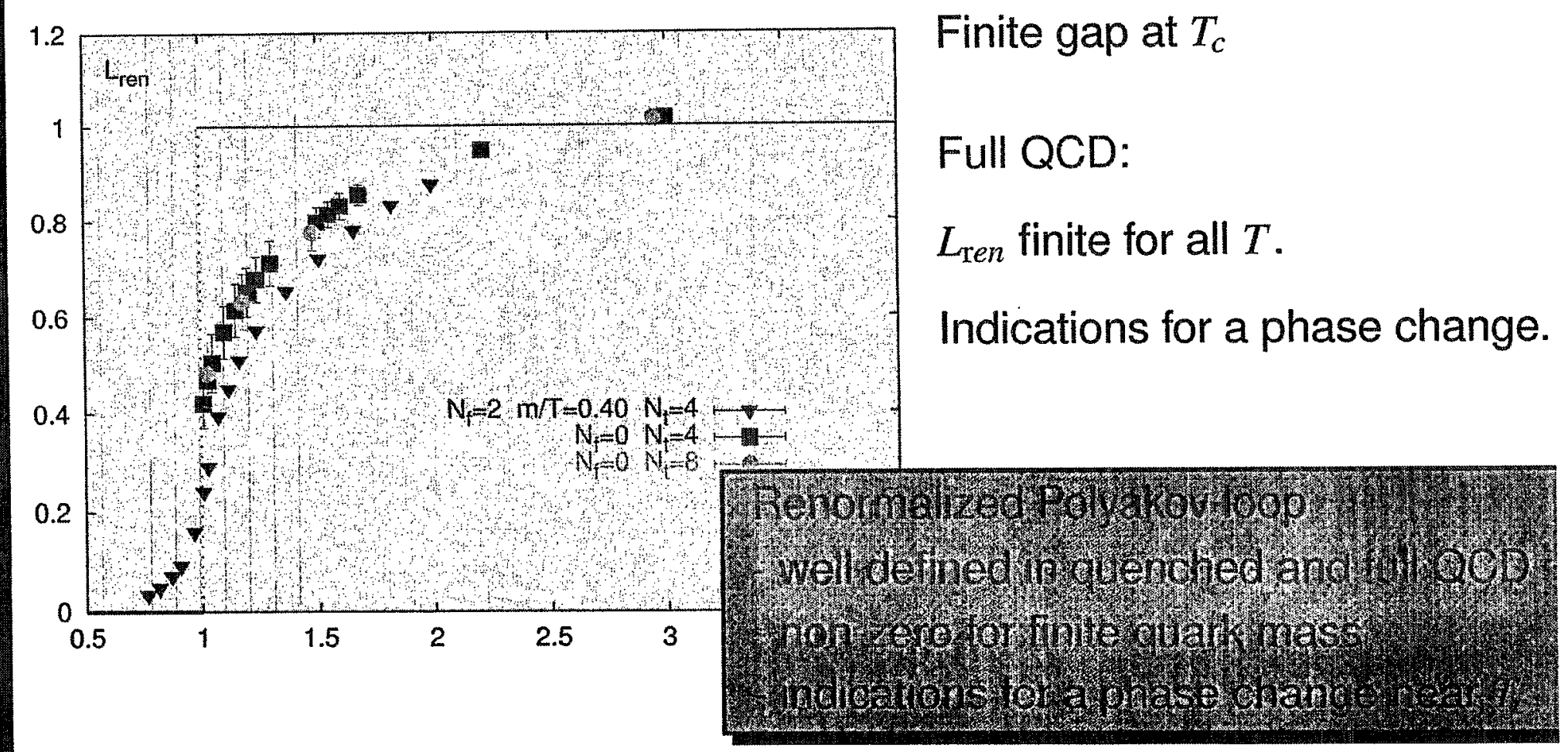




\section{BROOKHEWEN NATIONAL LABORATORY}

Static Quark-Antiquark Potentials at Finite Temperature with Asqtad improved fermions

by Peter Petreczky and Konstantin Petrov 


\section{Introduction}

GOALS:

- study thermodynamic potentials of a static quark-antiquark $(Q \bar{Q})$ pair

- determine free energies at infinite separation

- study order parameter

- glance at thermodynamics

- does it scale?

- what happens at short distances?

MEANS:

MODEL:

$N_{f}=3$ flavour QCD with Asqtad-improved staggered fermions

- introduced and advocated by MILC collaboration

- particulary suitable for studying thermodynamical properties

- good scaling behaviour

- 80 virtually independent configuration at high temperatures (made by MILC)

- 400 such in critical region

Singlet free energy of a static quark-antiquark is defined as [McLerran/Svetitsky '81]:

$$
F_{1}(|x|, T) / T=-\ln \left\langle\operatorname{Tr} L(\vec{x}) L^{\dagger}(0)\right\rangle
$$

- obviously non-gauge-invariant $\rightarrow$ perform calculations in the Coulomb gauge

- which is equivalent to using non-local gauge-invariant definition [Philipsen]

Should be renormalized:

- use zero-temperature potential for normalization [Karsch et al]

- by making all potentials equal to zero temperature one at smallest lattice spacing

- using Cornell potential with the scale $r_{0}=0.514 \mathrm{fm}$

$$
V(r)=\frac{0.4}{r}+1.2 \frac{r}{r_{0}^{2}}
$$




\section{Singlet potentials}

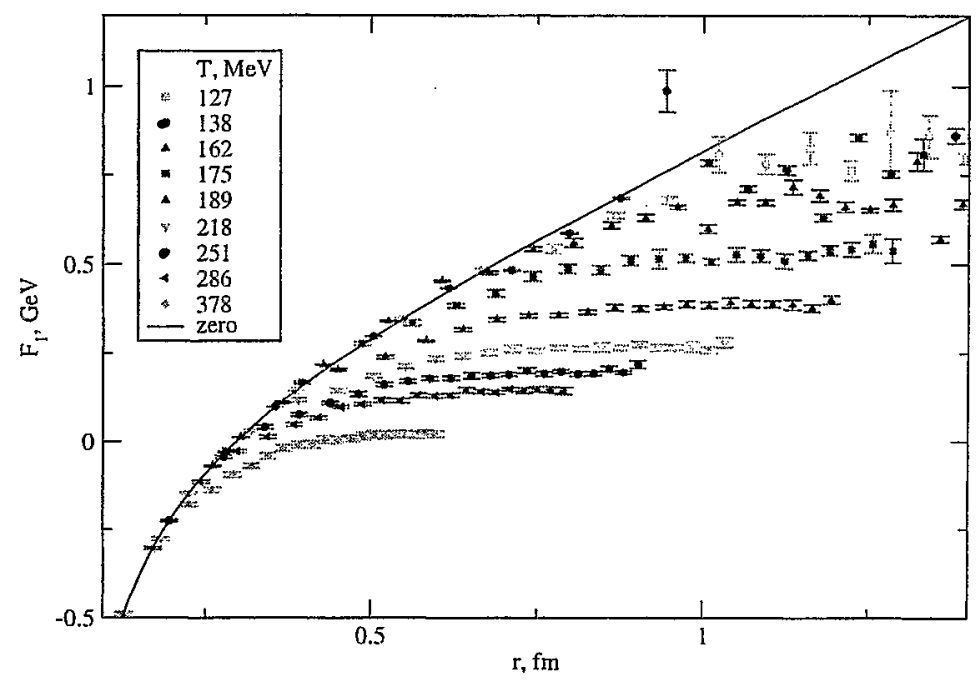

Singlet free energies:

- go to a constant for large distances

- for $T<150 \mathrm{MeV}$ this happens at $1 \mathrm{fm}$ $\longrightarrow$ string breaking

- at higher temperatures at distances which are decreasing as temperature increases $\longrightarrow$ screening

\section{Free and Internal Energy}

- extract asymptotic value by fitting to a constant

$$
F_{\infty}=\lim _{x \rightarrow \infty} F_{1}(|x|, T)
$$

- the corresponding enthropy

$$
S_{\infty}(T)=-\partial F_{\infty} / \partial T
$$

- and the internal energy

$$
U_{\infty}(T)=F_{\infty}(T)+T S_{\infty}(T)
$$

- at $T \rightarrow 0 U_{\infty} / 2$ gives the binding energy of a heavy-light meson (D,B-meson)

- at $T>0$ we can use the ansatz for the $T$-dependent $D, B$-meson masses.

$$
2 M_{D, B}(T)=U_{\infty}+2 m_{c, b}
$$


- $M_{D, B}$ does not decrease below $T_{c}$ (assuming $T_{c} \simeq 170 \mathrm{MeV}$ )

- this is consistent with Bielefeld results

Free Energy at infinite separation

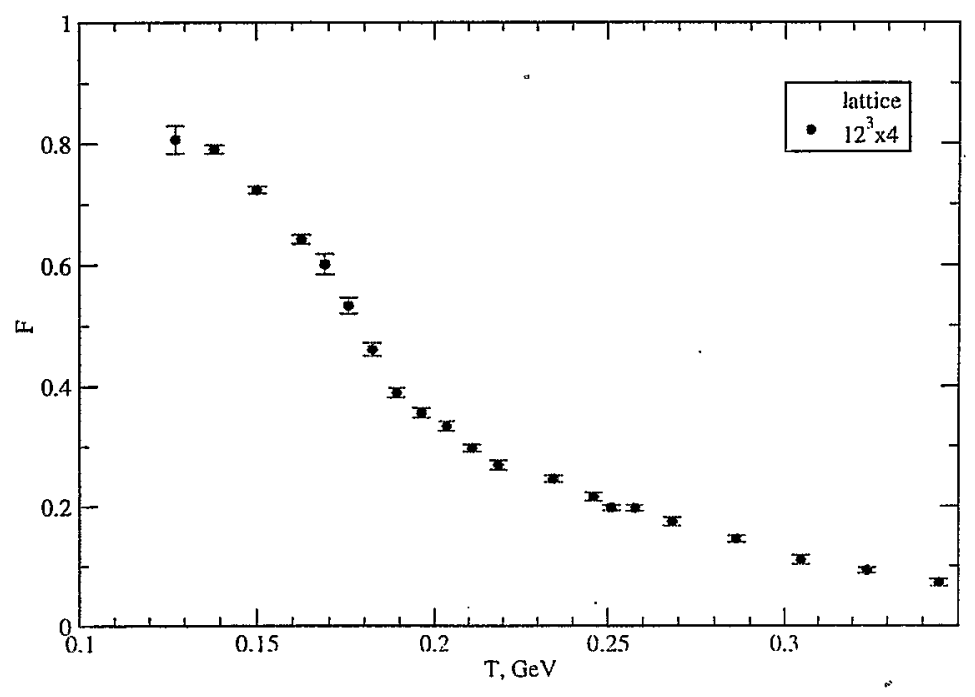

Internal Energy

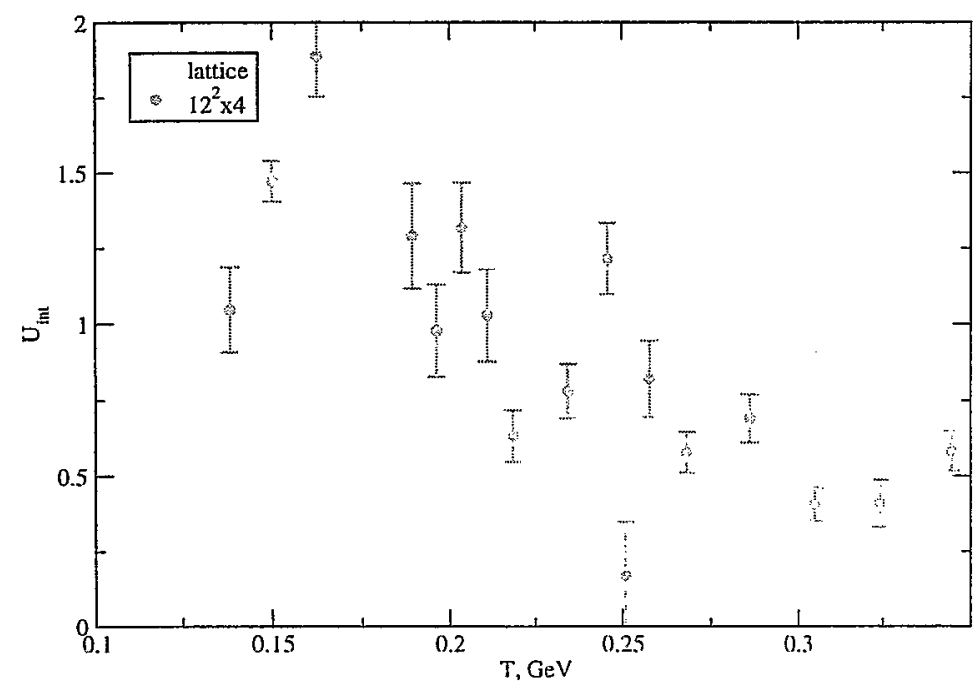




\section{Renormalized Order Parameter}

- used in a various potential models [e.g. Pisarski et al, Moscy et al]

- naive definition leads to bad scaling properties

- defined here as $L_{r e n}=\exp \left(-F_{\infty} / 2 T\right)$ [Karsch et al]

- other definitions availible [Pisarski et al]

Renormalized Polyakov Loop

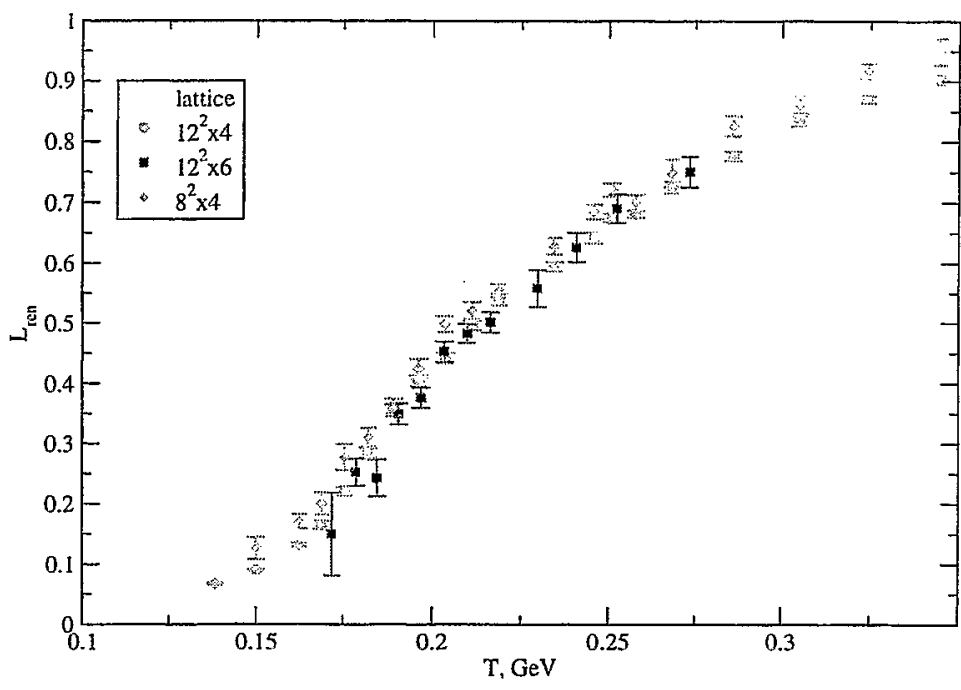

- seems to approach unity at high $\mathrm{T}$

- is $N_{\tau}$-independent

- pretty good finite size scaling behaviour

- little dependence on lattice spacing

$\longrightarrow$ does not become any steeper

- use bigger lattices to study short-distance behaviour

- no T-dependence is seen for distances below $0.4 \mathrm{fm}$

- however...

$$
\begin{aligned}
\alpha_{\dot{s}} & =\frac{3}{4} r^{2} \frac{\partial V}{\partial r} \\
& =\frac{3}{4}\left(0.4+\frac{1.2}{0.514^{2}} r^{2}\right)
\end{aligned}
$$


Running Coupling, $12^{3} \times 6$

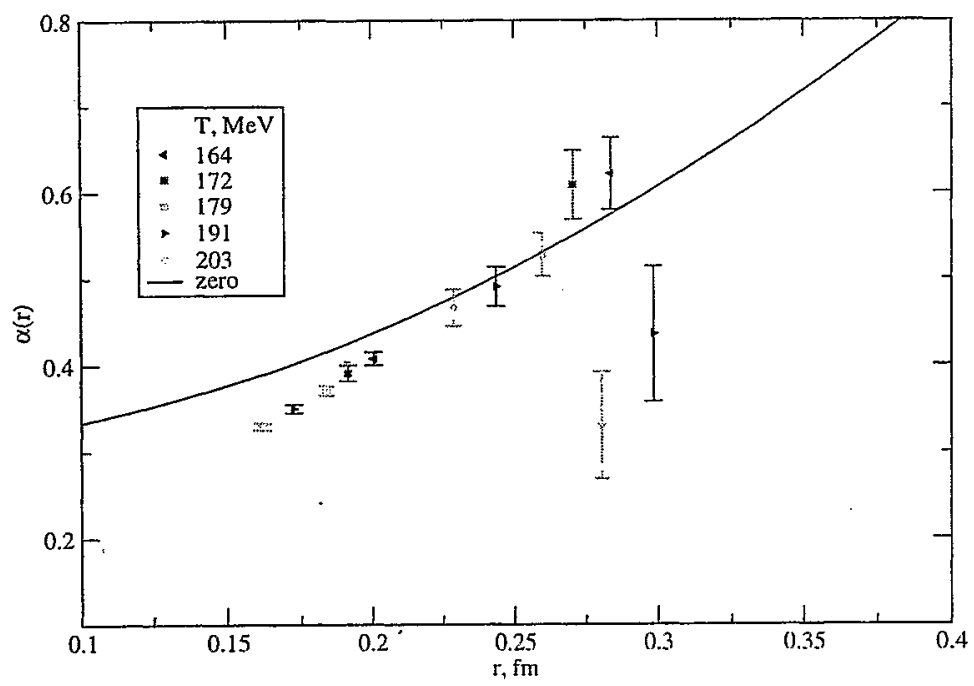

\section{Conclusions and Outlook}

- calculated the singlet free energy $F_{1}$ in three-flavour QCD

- at low temperatures we observe string breaking around $1 \mathrm{fm}$

- at higher temperatures string breaks earlier (screening)

- determined the renormalized order parameter for the deconfinement phase transition

- is independent of the lattice spacing

- $F_{\infty}$ decreases with temperature

- the internal energy $U_{\infty}$ does not decrease when approaching critical temperature from below

- implies that decrease in heavy-light meson masses is unlikely

- need mass dependence study, analyzing data for other quark masses

- $2+1 F$ study, in progress 


\title{
Mesonic Spectral Functions at Finite (High) Temperature
}

\author{
Masayuki Asakawa \\ Department of Physics, Kyoto University, Kyoto 606-8502 Japan
}

\begin{abstract}
We extract the spectral functions of $J / \psi$ and $\eta_{c}$ at finite temperature $(T)$ ranging from $T=0.78 T_{c}$ to $T=2.33 T_{c}$ by analyzing correlation functions on $32^{3} \times(32-96)$ anisotropic lattices with the maximum entropy method (MEM) ( $T_{c}$ is the critical temperature of deconfinement). In the analysis, we put special emphasis on the importance of (I) the MEM error analysis of the resultant spectral functions and (II) the study of the sensitivity of the spectral functions to the number of temporal data points adopted in MEM. We find that $J / \psi$ and $\eta_{c}$ survive as distinct resonances in the plasma even up to $T \simeq 1.62 T_{c}$ and that they eventually dissociate between $1.62 T_{c}$ and $1.70 T_{c}$. This result passes the above two tests. The iraplication of this result in the physics of high energy heavy ion collisions is also discussed.
\end{abstract}




\section{Result for V channel $(\mathrm{J} / \psi)$}

$\stackrel{\infty}{\circ}$

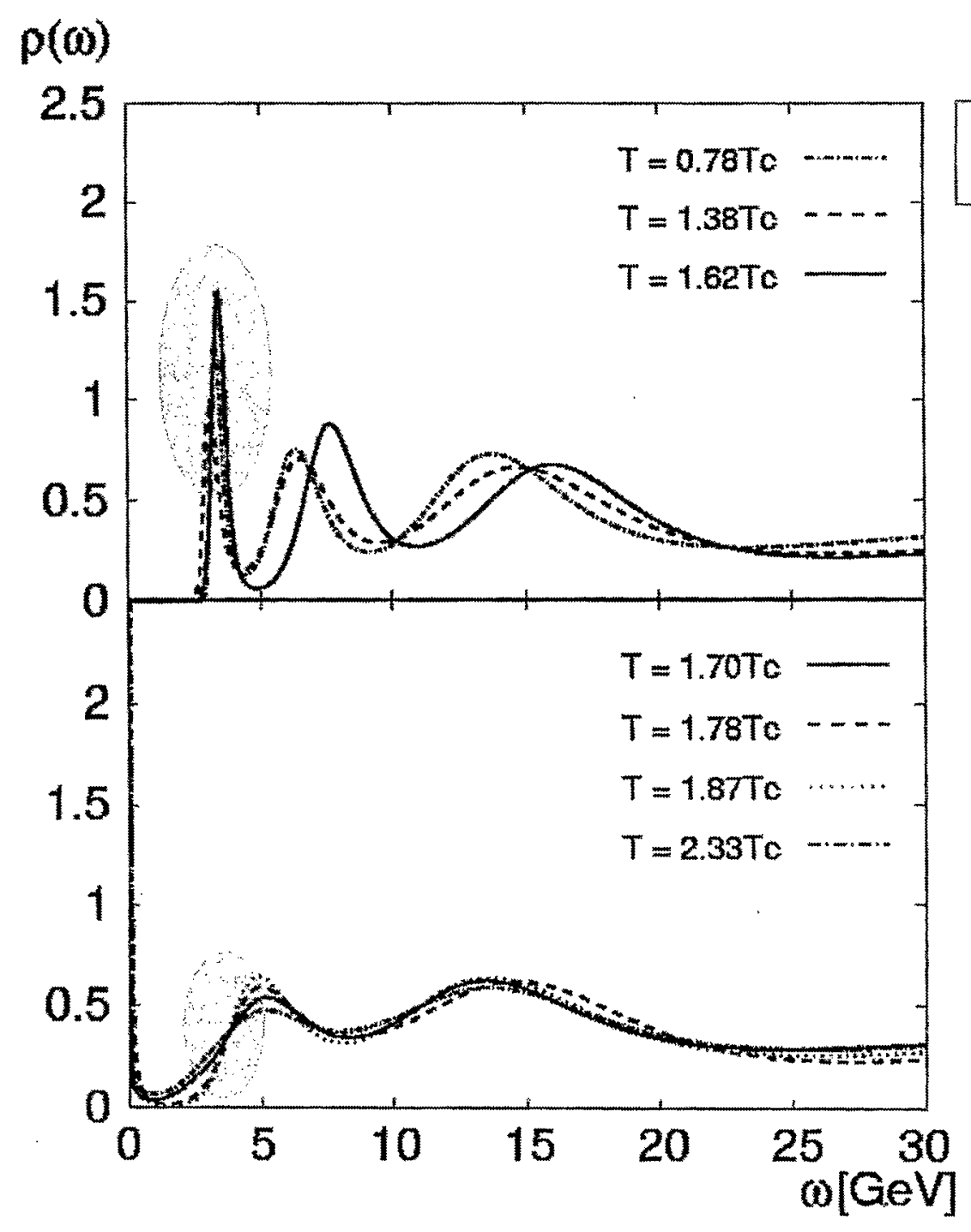

$$
A(\omega)=\omega^{2} \rho(\omega)
$$

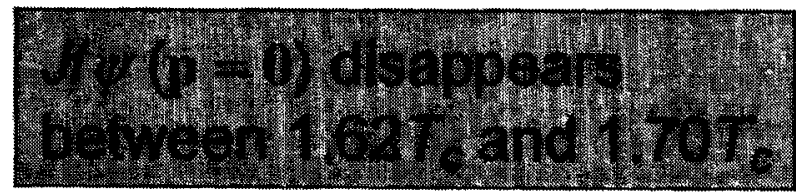

M. Asakawa (Kyoto University) 


\section{Result for PS channel ( $\left.\eta_{c}\right)$}

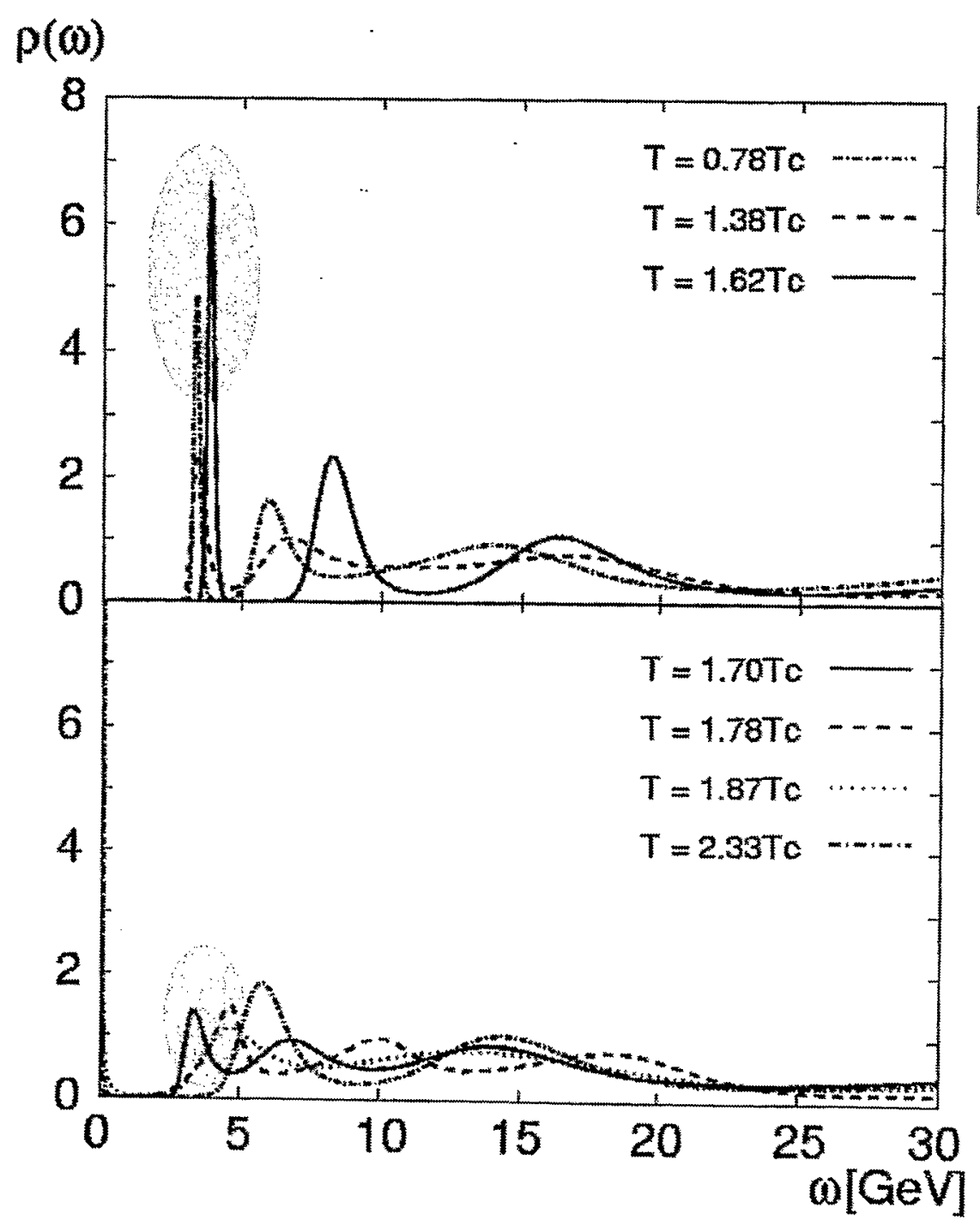

$$
A(\omega)=\omega^{2} \rho(\omega)
$$

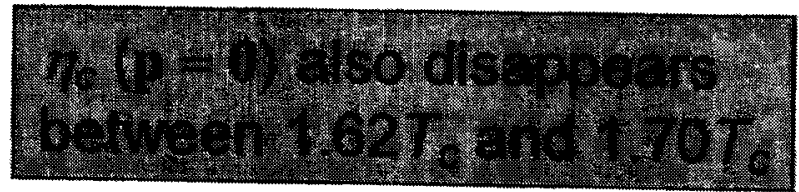

M. Asakawa (Kyoto University) 


\section{Statistical Significance Analysis for $\mathrm{J} / \psi$}

\section{Statistical Significance Analysis = Statistical Error Putting}

㕣

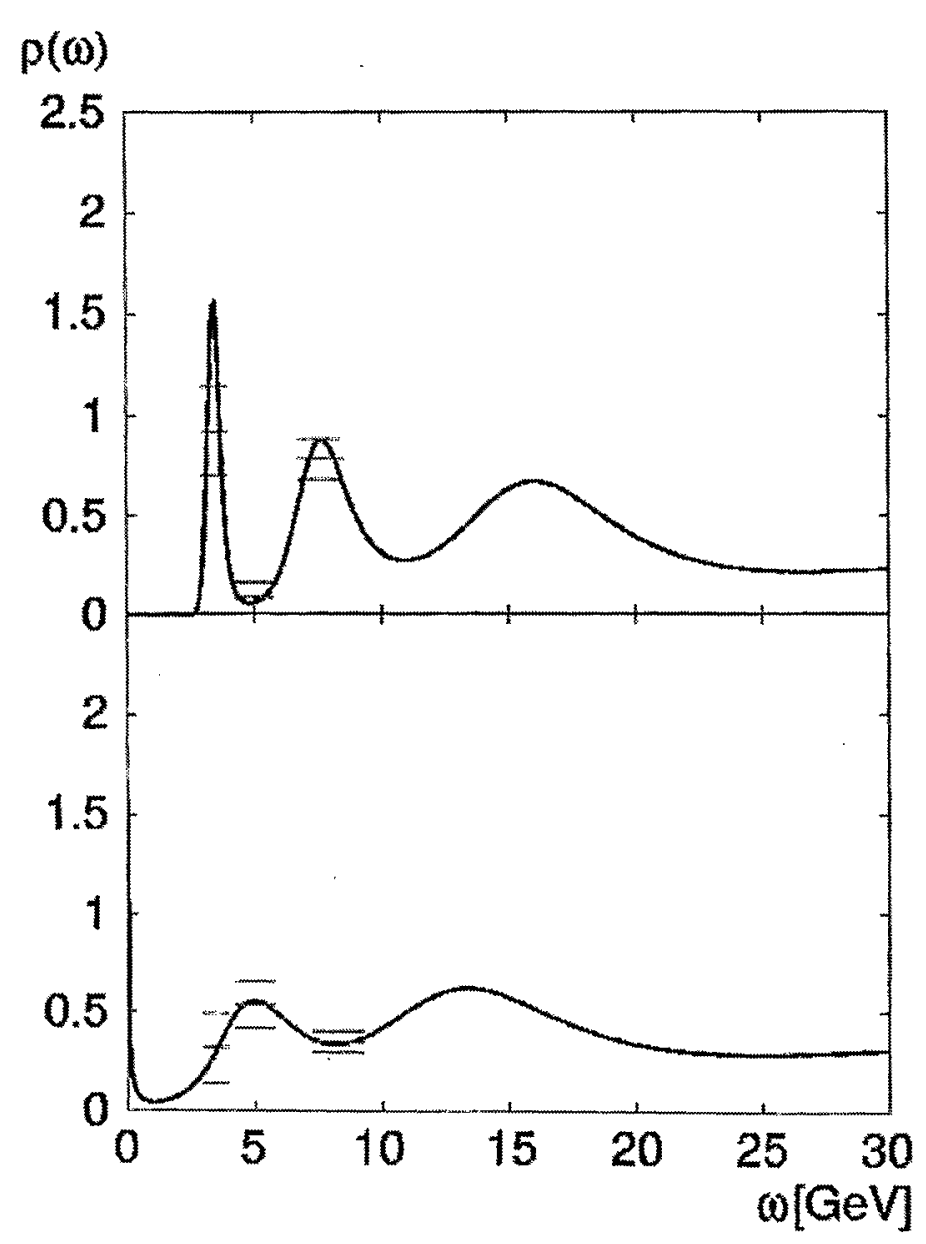

$$
\begin{aligned}
& T=1.62 T_{C} \\
& T=1.70 T_{C}
\end{aligned}
$$




\section{Dependence on Data Point Number (1)}

\section{Data Point \# Dependence Analysis = Systematic Error Estimate}
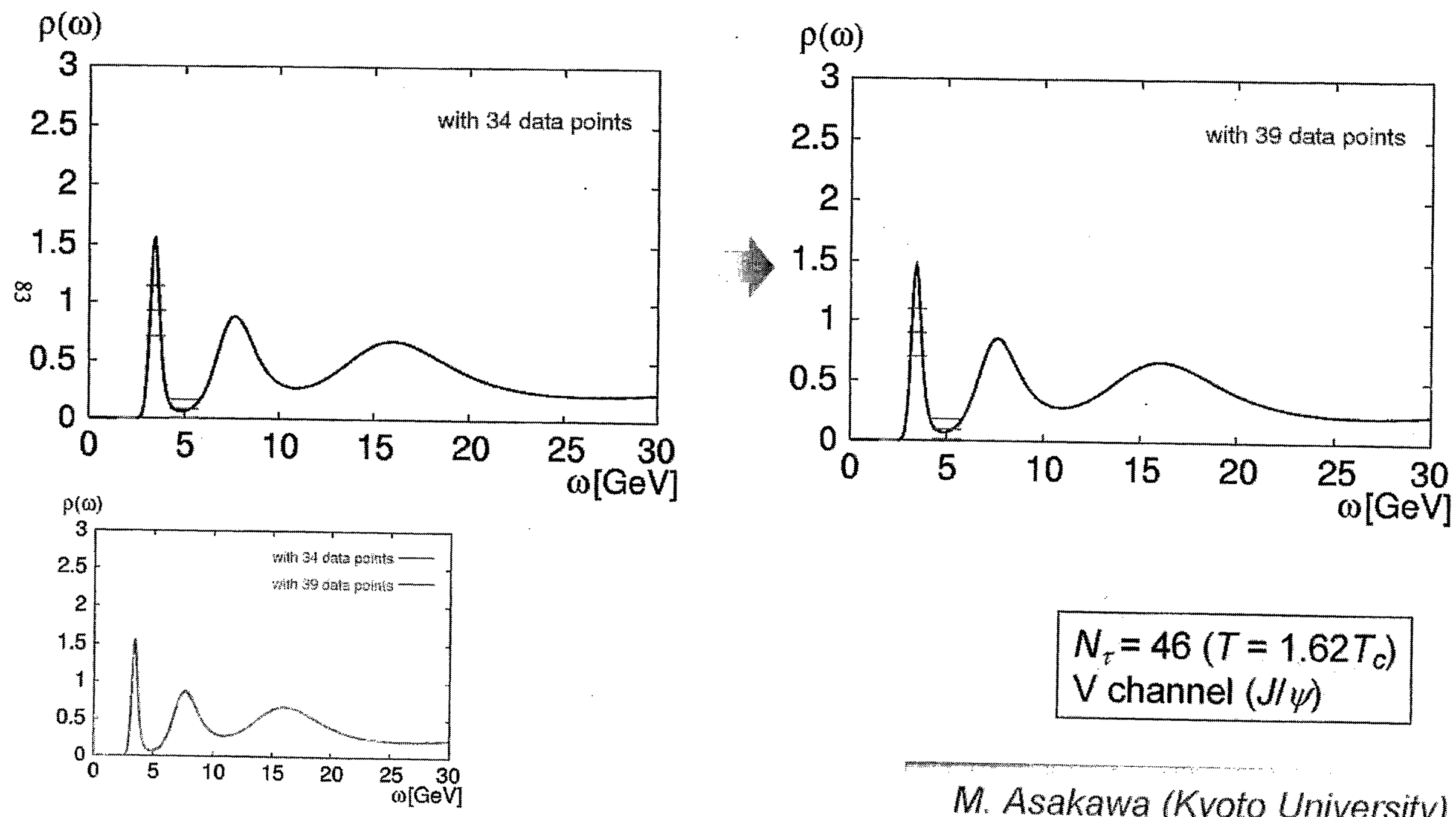

$$
\begin{aligned}
& N_{\tau}=46\left(T=1.62 T_{c}\right) \\
& V \text { channel }(J / \psi)
\end{aligned}
$$




\section{Summary and Perspectives}

- Spectral Functions in QGP Phase were obtained for heavy quark systems at $\mathbf{p}=\mathbf{0}$ on large lattices at several $T$

- Both Statistical and Systematic Error Estimates have been carefully carried out

- It seems $J / \psi$ and $\eta_{C}(\mathbf{p}=\mathbf{0})$ remain in QGP up to $\sim 1.6 T_{C}$

- Sudden Qualitative Change between $1.62 T_{C}$ and $1.70 T_{C}$

1: $~ 34$ Data Points look sufficient to carry out MEM analysis on the present Lattice and with the current Statistics (This is Lattice and Statistics dependent)

. Physics behind is still unknown

Further study needed for better understanding of QGP and Hadronic Modes in QGP!

- Recent RHIC data suggest strong correlation among partons at hadronization 


\title{
Charmonia in Finite Temperature Quenched QCD
}

\author{
Saumen Datta \\ Fakultät für Physik, Universität Bielefeld, D33605 Bielefeld, Germany \\ Based on work done with Frithjof Karsch, Peter Petreczky and Ines Wetzorke
}

I will present results from a lattice study of charmonia in hot gluonic plasma, for temperatures upto three times the deconfinement transition temperature $T_{c}$. I first discuss the high frequency structure of lattice (wilson) fermions, and argue that in order to extract the low energy physics at high temperatures from correlators measured on lattice at high temperatures, it is important to provide information about the high frequency structure. An analysis of the correlators show that the $\chi_{c_{0}}$ and $\chi_{c_{1}}$ suffer serious modifications induced by the hot medium already by $1.1 T_{c}$. The $\eta_{c}$ and $J / \psi$, on the other hand, survive till quite deep in the plasma. Upto $1.5 T_{c}$ little significant reduction of the peak strength is seen in these channels. At higher temperatures, the peak gets gradually weakened; however, a significant peak is seen in these channels till $\sim 2.25$ $T_{c}$. The spectral function at finite temperature shows some change on crossing $T_{c}$; in particular, the in-medium dispersion relation seems to change from the relativistic one. This results in an increase in the screening mass, extracted from long distance decay rate of spatial correlators, with temperature. 

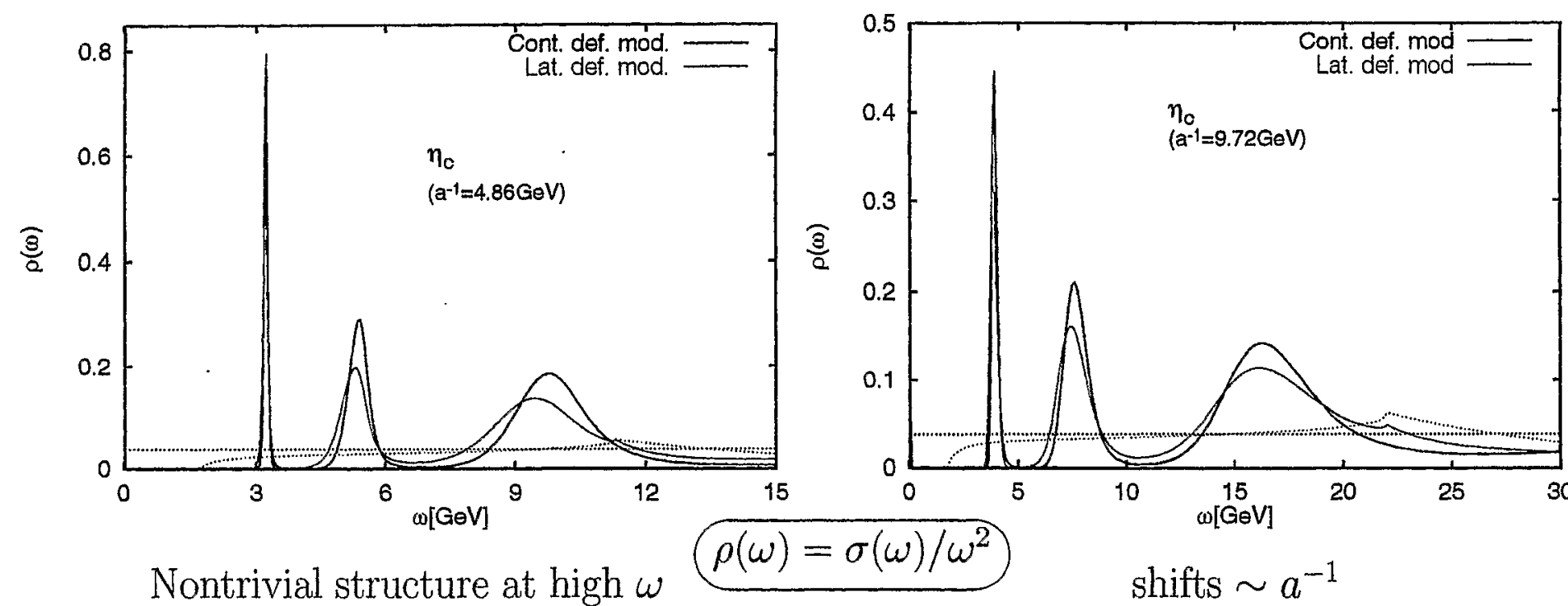

Analysis strategy:

- Supply high $\omega$ peak structure as prior information peak structure at high $\omega \sim \omega^{2}$ at low $\omega$

- See if spectral function at low temperature explains data at high temp.

Reconstructed correlator $G_{\text {recon, } T^{*}}(\tau, T)=\int d \omega \sigma\left(\omega, T^{*}\right) K(\omega, \tau, T)$

Compare with $G(\tau, T)$ 
$1 \mathrm{P}$ states above $T_{c}$
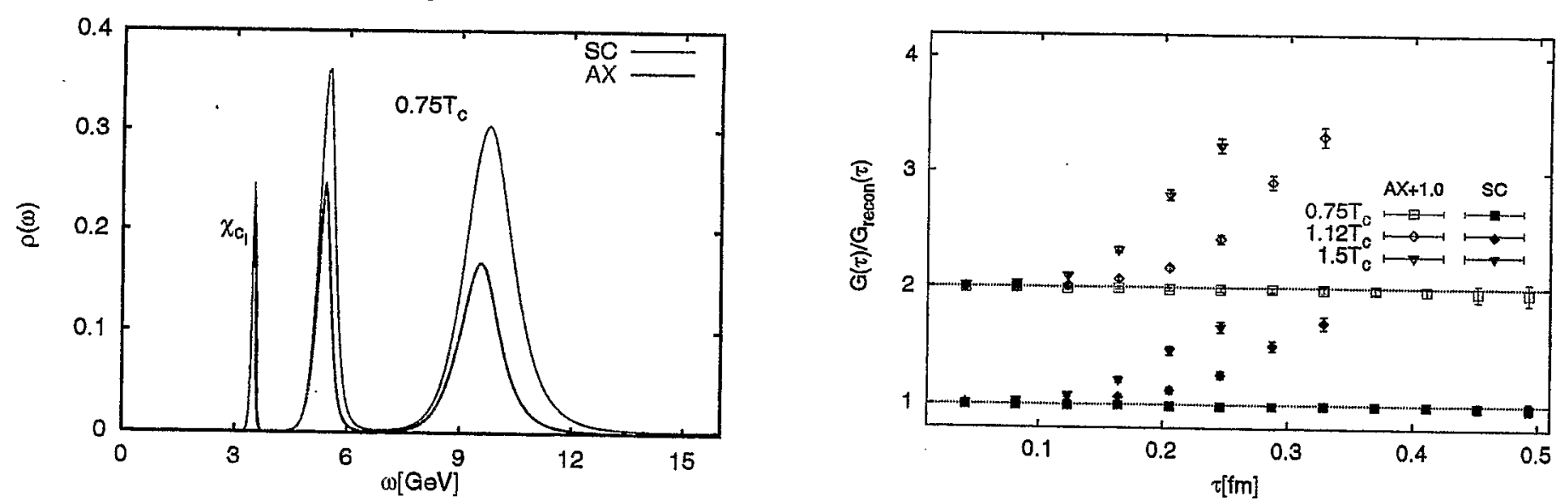

$\stackrel{\infty}{3}$
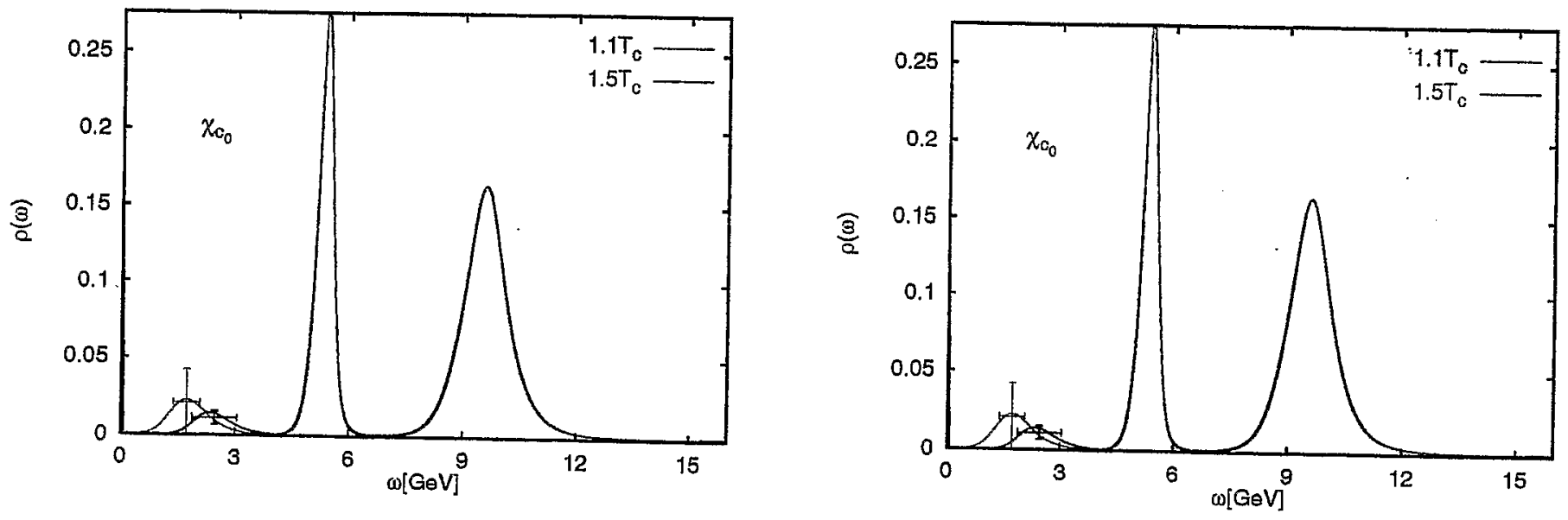

Serious system modification already at $1.1 T_{c}$

Changes consistent with dissolution 

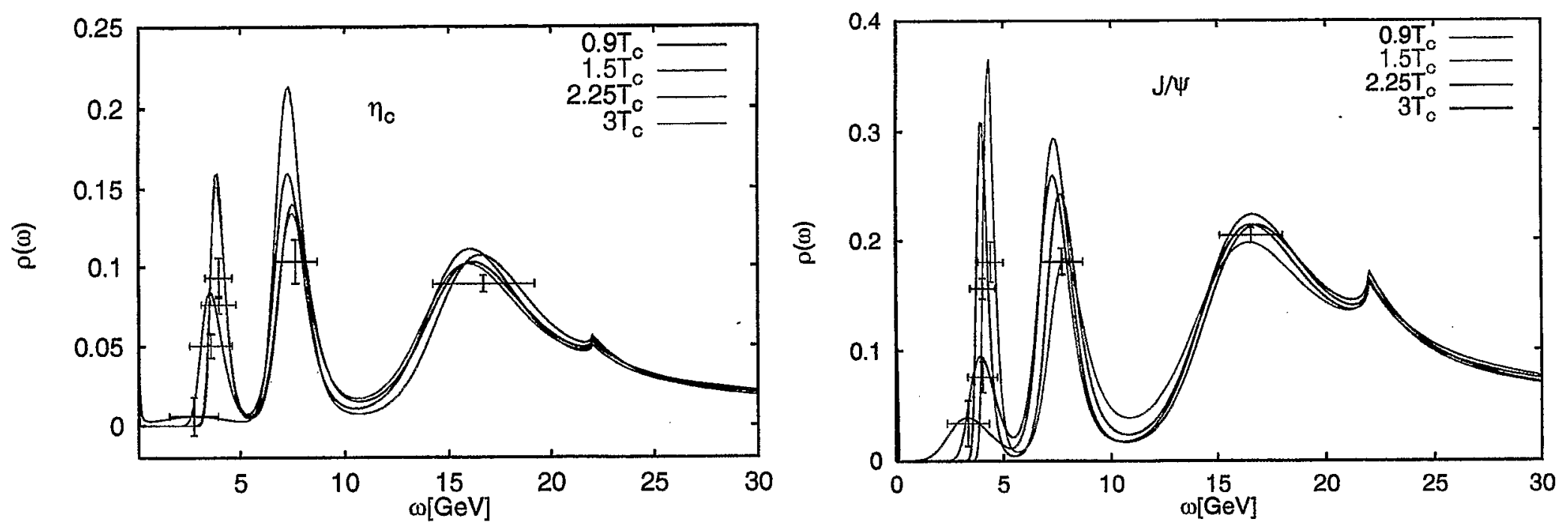

$1 \mathrm{~S}$ States survive upto $2.25 T_{c}$

- $\eta_{c}$ shows no change upto $1.5 T_{c}$

- Weakening (and possibly broadening) at $2.25 T_{c}$

- No significant resonance seen at $3 T_{c}$
- $J / \psi$ shows no weakening upto $1.5 T_{c}$

- Weakening (and possibly broadening) at $2.25 T_{c}$

- No significant resonance seen at $3 T_{c}$ 
Screening masses from spatial correlators:

information about spectral function at finite momenta

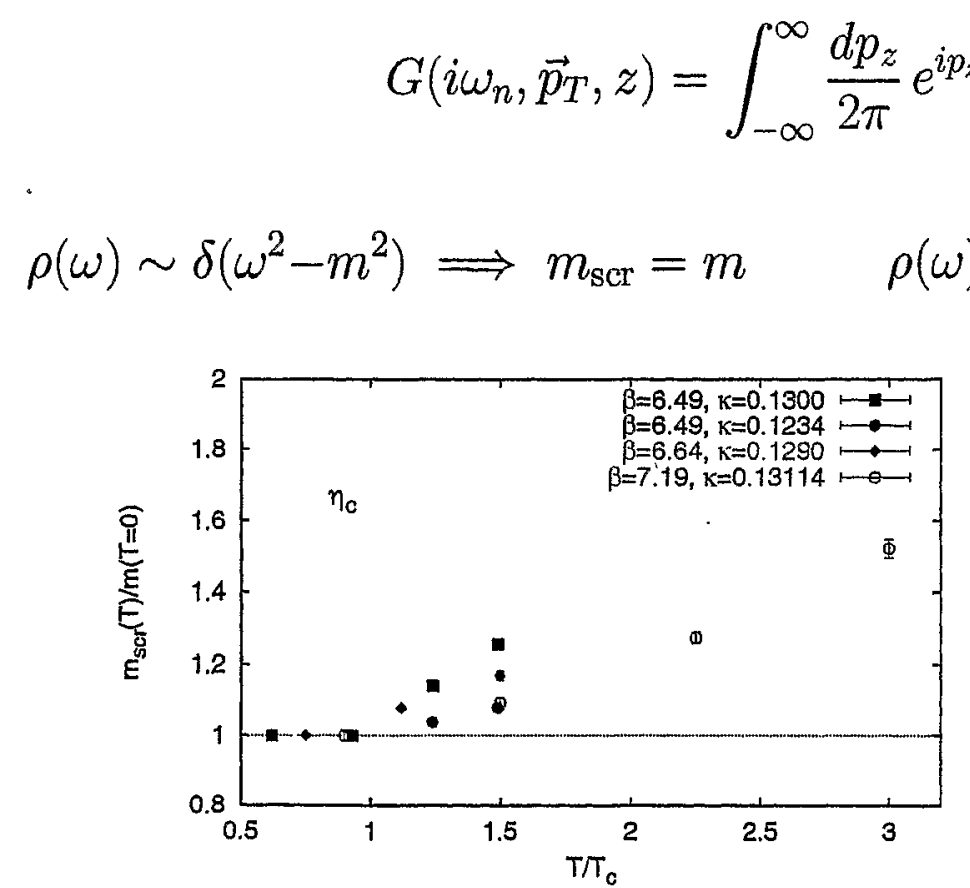

$J / \psi$ moving in heatbath frame sees more energetic gluons

collision broadening and weakening?

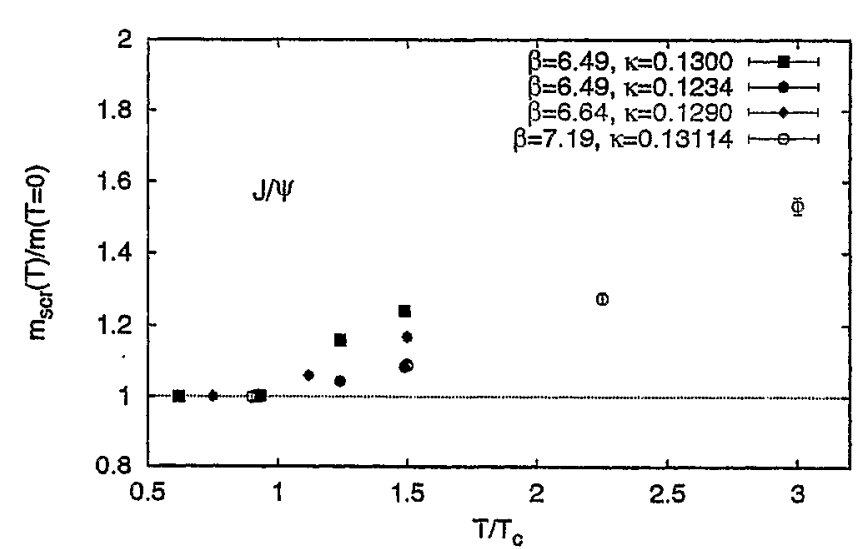

Change in dispersion relation

$$
\begin{aligned}
& \omega^{2}(\vec{p}, T) \sim m^{2}(T)+A^{2}(T) \vec{p}^{2} \\
& \Longrightarrow m_{\mathrm{scr}}(T)=m_{\mathrm{pole}}(T) / A(T)
\end{aligned}
$$



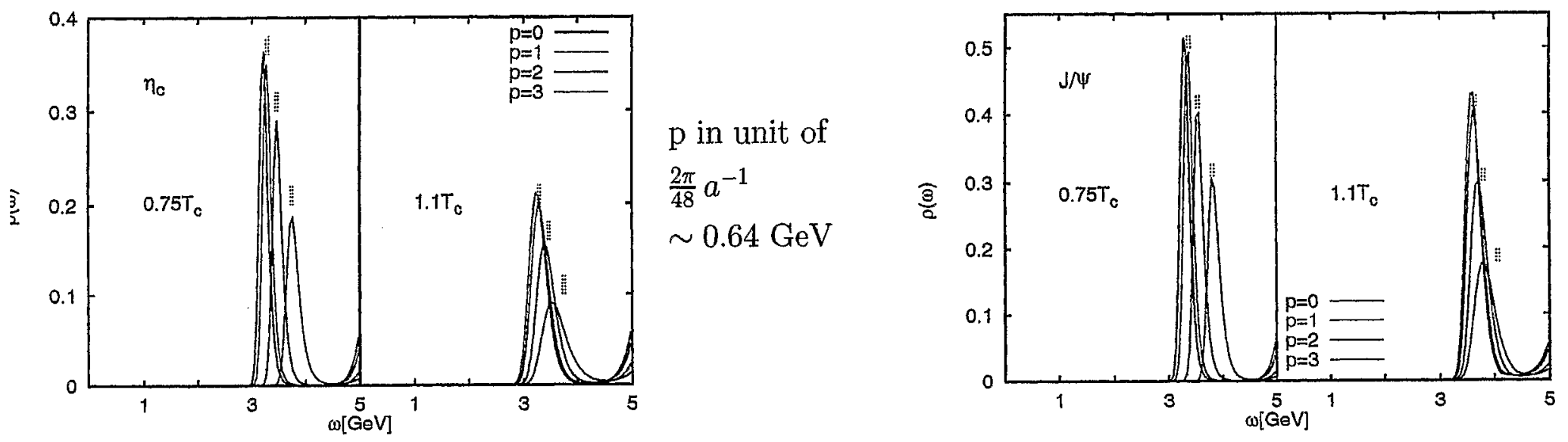

8

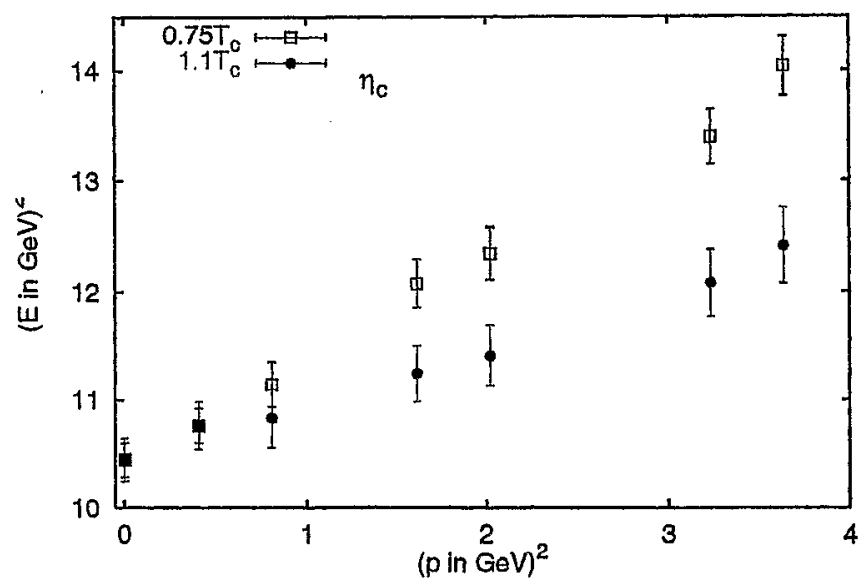

Change in

dispersion relation above $T_{c}$

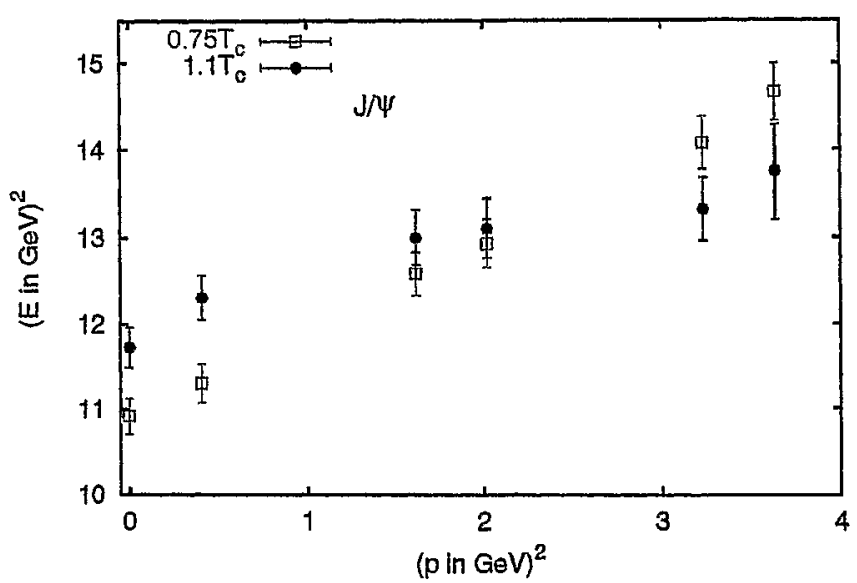




\title{
Properties of the glueball at finite temperature from anisotropic lattice QCD
}

\author{
N. Ishii and H. Suganuma \\ Faculty of Science, Tokyo Institute of Technology
}

\begin{abstract}
The thermal properties of the $0^{++}$glueball are studied using $\mathrm{SU}(3)$ anisotropic lattice QCD with $\beta_{\text {lat }}=6.25$, the renormalized anisotropy $\xi \equiv a_{s} / a_{t}=4$ over the lattice of the size $20^{3} \times N_{t}$ with $N_{t}=24,26,28,30,33,34,35,36,37,38,40,43,45,50,72$ at the quenched level. (These $N_{t}$ correspond to $T=390-130 \mathrm{MeV}$, which contain the case above the critical temperature $T_{c} \simeq 280 \mathrm{MeV}$. However, in this talk, we focus on the thermal effects below $T_{c}$.) We adopt APE smearing to improve the glueball operator so as to enhance the overlap to the lowest $0^{++}$glueball peak. We construct the temporal correlators of these smeared glueball operators at each temperature using 5,500-9,900 gauge configurations at each temperature $T$. We perform a fit analysis of these glueball correlators by adopting an an extended ansatz (Breit-Wigner ansatz) in order to take into account the possible appearance of the thermal width at finite temperature. We find a significant thermal effect on the glueball correlators near the critical temperature, i.e., the glueball peak acquires a huge thermal width as $\Gamma\left(T_{c}\right) \simeq 300 \mathrm{MeV}$ and shows a modest reduction in the peak center as $\omega_{0}\left(T_{c}\right) \simeq 100 \mathrm{MeV}$. We finally discuss the possible implications of these results.
\end{abstract}

\section{References}

[1] N. Ishii, H. Suganuma and H. Matsufuru, Phys. Rev. D66 (2002) 014507.

[2] N. Ishii, H. Suganuma and H. Matsufuru, Phys. Rev. D66 (2002) 094506.

[3] N. Ishii and H. Suganuma, Eur. Phys. J. A17 (2003) 77. 


\section{Small Tc v.s. Heavy glueball mass}

In quenched QCD,

$$
T_{C} \cong 260-280 \mathrm{MeV}<<m_{G} \cong 1500-1700 \mathrm{MeV}
$$

$\Rightarrow$ Thermal excitation of the glueball is suppressed in the confinement phase by a very strong statistical factor as

$$
\exp \left(-\sqrt{m_{G}{ }^{2}+\vec{p}^{2}} / T\right)<e^{-m_{G} / T_{C}} \cong 0.00207
$$

The glueball density is very small even near Tc:

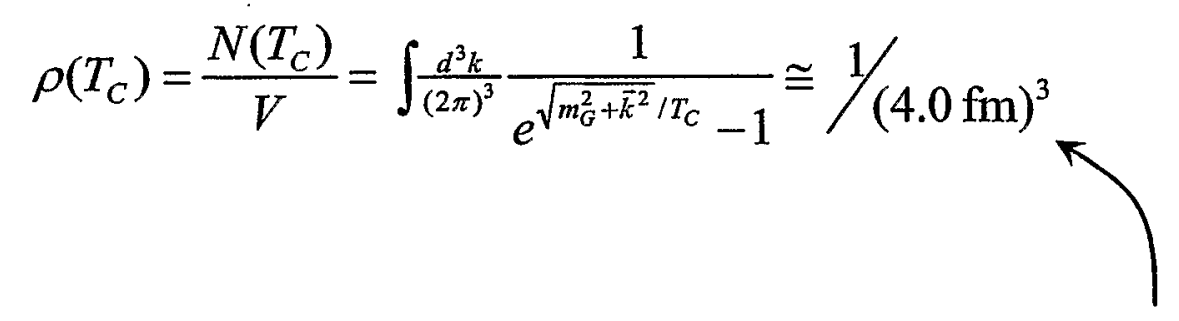

This density is considered to be TOO SMALL:

- The glueball is considered to have a rather small size !

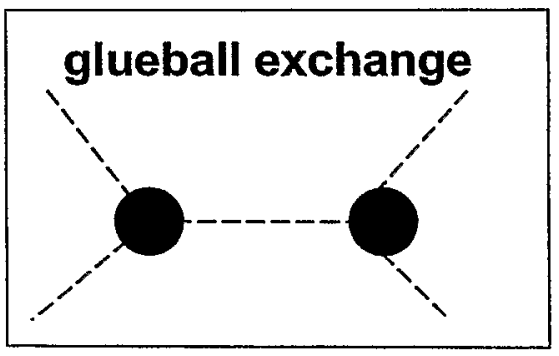

$$
r \leq 0.2-0.4 \mathrm{fm}
$$

- The interactions among glueballs are expected to be very short-ranged, since the interaction is mediated by rather heavy glueball $\left(\mathrm{m}_{\mathrm{G}}>1.5 \mathrm{GeV}\right)$ exchanges. 
One possible answer would be

\section{glueball (pole) mass reduction (and/or swelling of the glueball size)}

near the critical temperature $T_{C}$

In fact, the glueball mass reduction near $T_{C}$ is suggested by

H. Ichie et al., PRD52, 2944 (1995)

based on the effective model studies (dual Ginzburg-Landau model).

In this talk, we report the anisotropic lattice QCD study of the thermal glueball near the critical temperature.

References:

- N. Ishii et al., PRD66,014507(2002)

- N. Ishii et al., PRD66,094506(2002)

- N. Ishii et al., EPJA17,77(2003) 
The glueball correlator at high temperature $(\mathrm{T}=253 \mathrm{MeV}<\mathrm{Tc})$

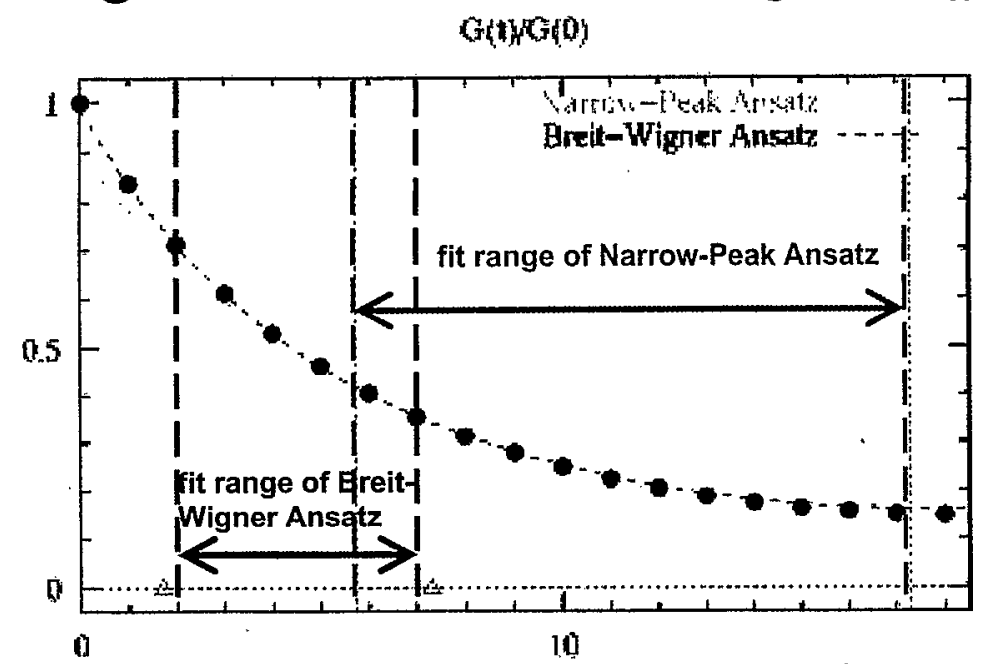

Appropriate smearing is adopted.

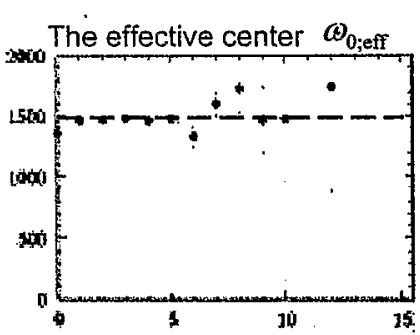

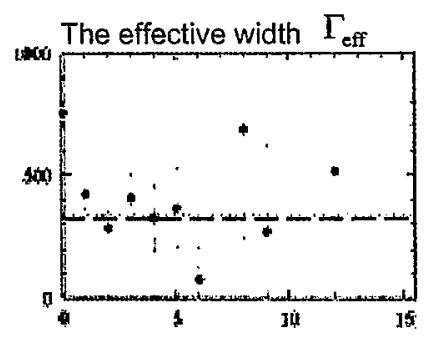

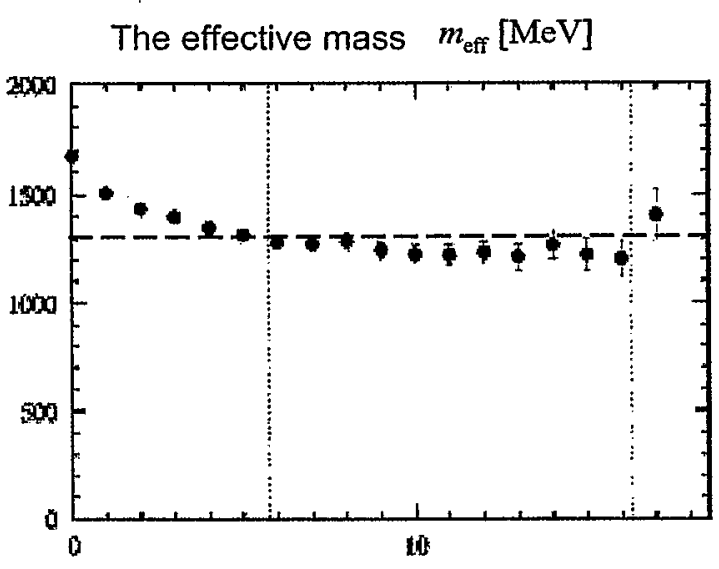

The narrow-peak ansatz fails to fit the lattice QCD data around $t=0$.

The Breit-Wigner ansatz fits the lattice QCD data in the whole region rather well. 


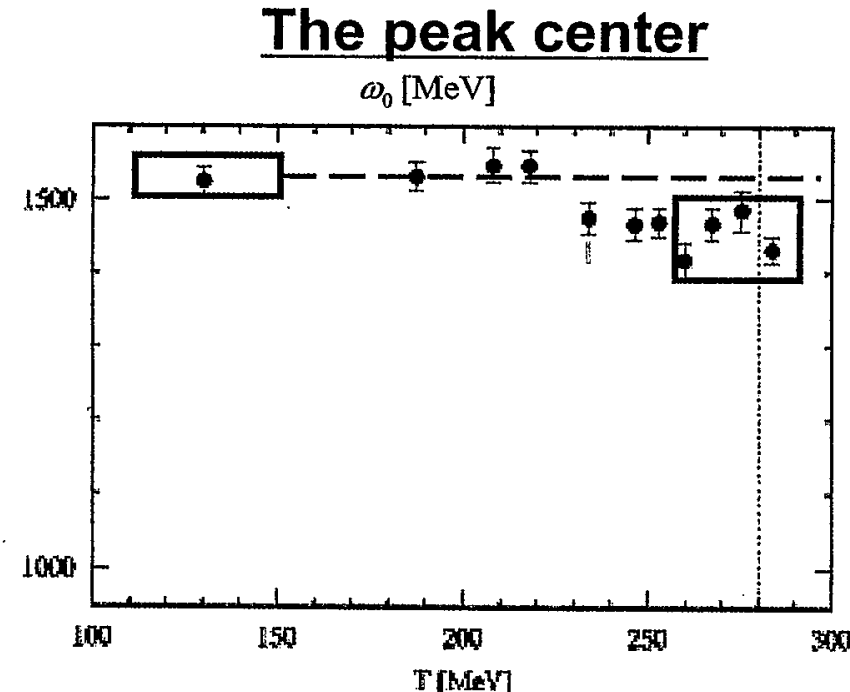

భi

The polemass from narrow peak ansatz

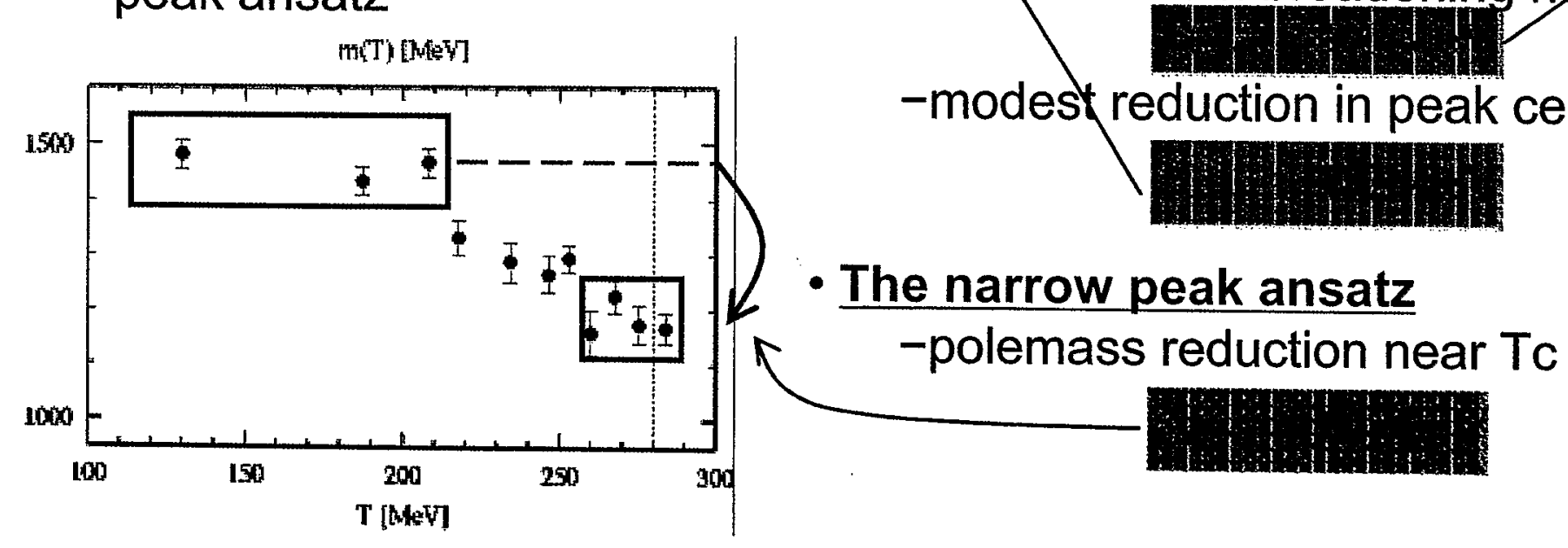

The thermal width

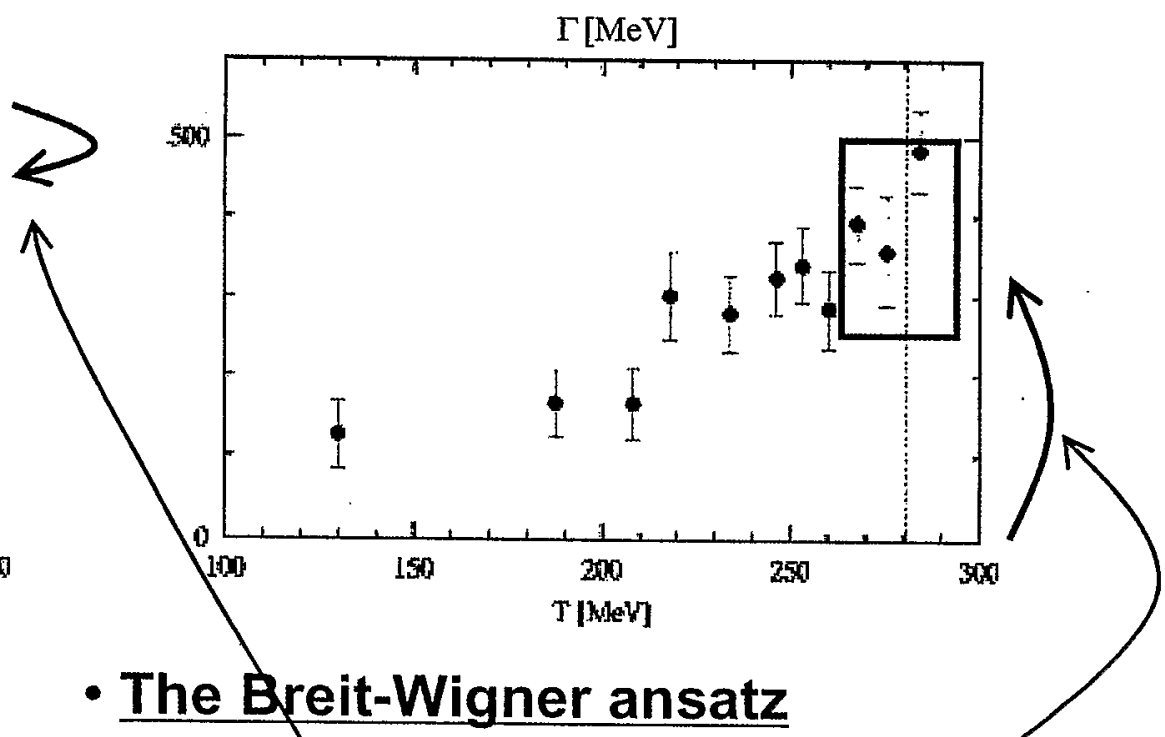

- thermal width broadening near Tc ter 


\section{Summary / Discussion}

1. We have constructed the temporal $\mathbf{0}^{++}$glueball correlators at finite temperature using 5,500-9,900 gauge configurations generated by $\mathrm{SU}(3)$ anisotropic lattice QCD at quenched level.

2. We have performed an advanced analysis adopting Breit-Wigner ansatz for the spectral function in order to take into account the possible appearance of the thermal width at finite temperature.
a. A significant thermal width broadening near Tc:
$\Gamma\left(T_{\mathrm{C}}\right) \cong 300 \mathrm{MeV}$
b. A modest reduction in peak center near Tc:
$\Delta \omega_{0}\left(T_{\mathrm{C}}\right) \cong 100 \mathrm{MeV}$
c. No significant thermal effects in the glueball size.

3. What is the origin of such a huge thermal width ?

a. It is not thermally excited lowest-lying glueballs. (Too small glueball density even at Tc)

b. The contributions of bound state glueballs listed in C.J.Morningstar et al., PRD60,034509('99) is estimated to be not significant. (They are too heavy.)

c. We have to resort to higher energy resonace modes trusting in the sufficient increase in the number of modes. $\Rightarrow$ Hagedorn's picture, Resonance gas model, ... ?

4. Future Plans:

Glueball correlations above Tc, Experimental observability, Extension to the unquenched QCD calculations, etc. 


\title{
Finite Temperature Meson Correlations of Light Quarks
}

\author{
E. LAERMANN \\ Fakultät für Physik, Universität Bielefeld, D-33615 Bielefeld, Germany
}

\begin{abstract}
Results from lattice QCD simulations at finite temperature are presented. They have been obtained from a non-perturbatively improved Wilson fermion action on quenched configurations. The observables discussed are spatial as well as temporal correlation functions for light quarks. From those, screening masses and spectral densities have been extracted, the latter through the use of the Maximum Entropy Method. Special emphasis is laid on the discussion of finite volume and finite lattice spacing effects.
\end{abstract}


at $T=0$ obtain masses and amplitudes, e.g. $m_{\pi}, f_{\pi}$ from

$$
G_{\pi}^{T}(\tau) \underset{\tau \rightarrow \infty}{\longrightarrow} f_{\pi}^{2} m_{\pi} \exp \left(-m_{\pi} \tau\right)
$$

Obstacles at $T \neq 0$ :

- existence of bound states, resonances etc. usually unknown

$\leadsto$ try to establish spectral density

- limited physical extension $1 / T$ in temporal direction

$\leadsto$ spatial correlations

- limited number of data points $N_{\tau}$ in temporal direction

$\sim$ anisotropic lattices

- finite size $\quad L T=N_{\sigma} / N_{\tau}$

- finite lattice spacing $a T=1 / N_{\tau}$ 
vice versa ( $a \rightarrow 0$ first)

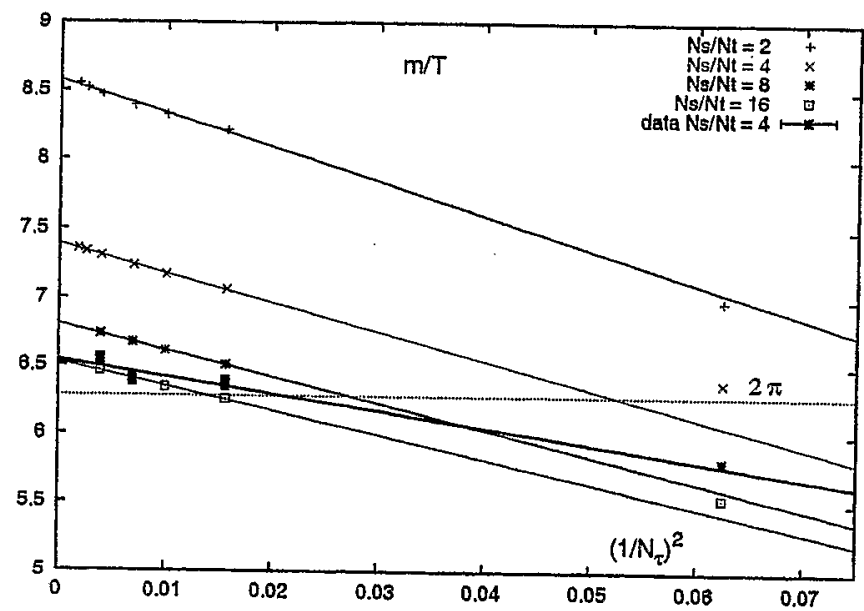

finite lattice spacing effect

$$
\begin{array}{r}
m_{\text {screen }}(L, a)=2 \pi T \alpha(L) \\
\star\left(1-\gamma_{a} \frac{\pi^{2}}{3} \frac{1}{N_{\tau}^{2}}\right)
\end{array}
$$

again data at $3 T_{c}$ :

- slightly less affected

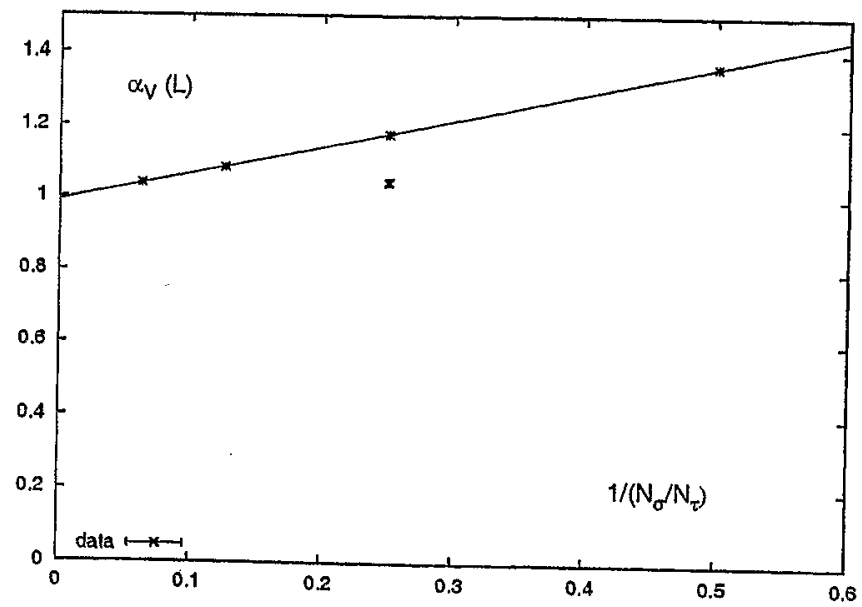

finite volume effect

$$
\alpha(L)=1+\gamma_{V} \frac{2}{\pi} \frac{N_{\tau}}{N_{\sigma}}
$$


screening masses below $T_{c}$

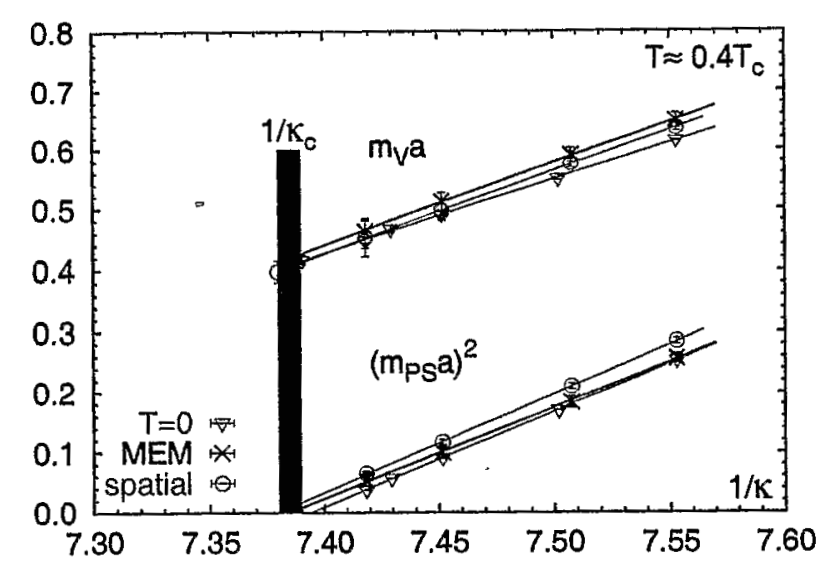

comparison with $T=0: \quad$ [Göckeler et al.]

- at $0.4 T_{c}$ no difference

- masses from MEM : likewise

\section{summary:}

$T<T_{c}$ : only weak $T$ dependence slight finite size in $\rho$ at $0.9 T_{c}$ possible

$T>T_{c}$ : largest volume data only

normalized to same lattice size free case might overestimate difference to $2 \pi$

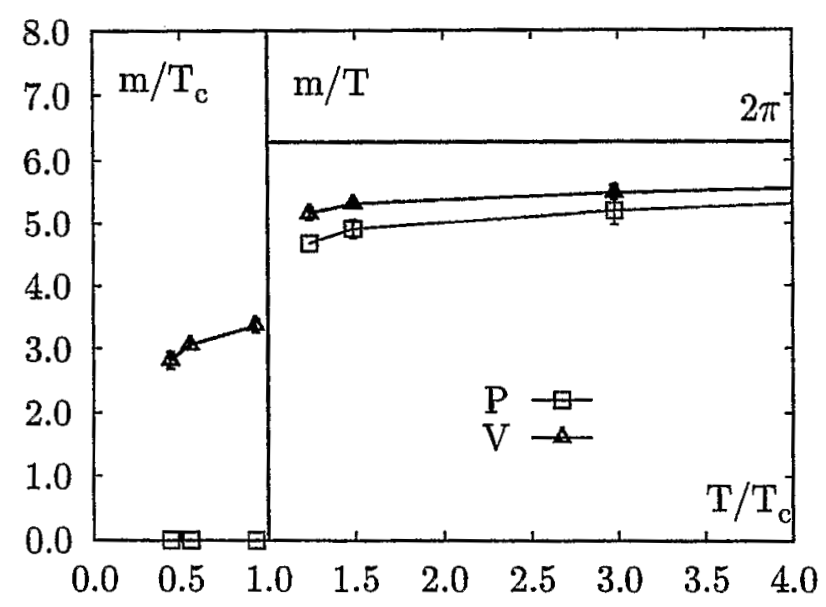


MEM results below $T_{c}$ : compare with screening masses
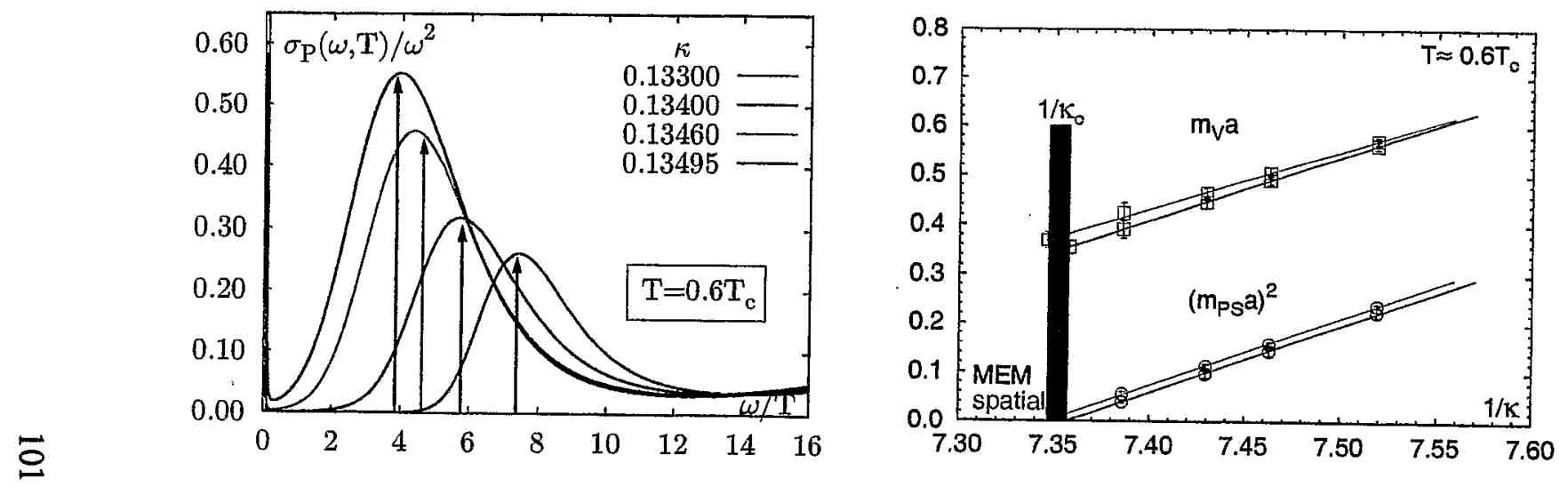

$T=0.6 T_{c}$

no siginificant differences
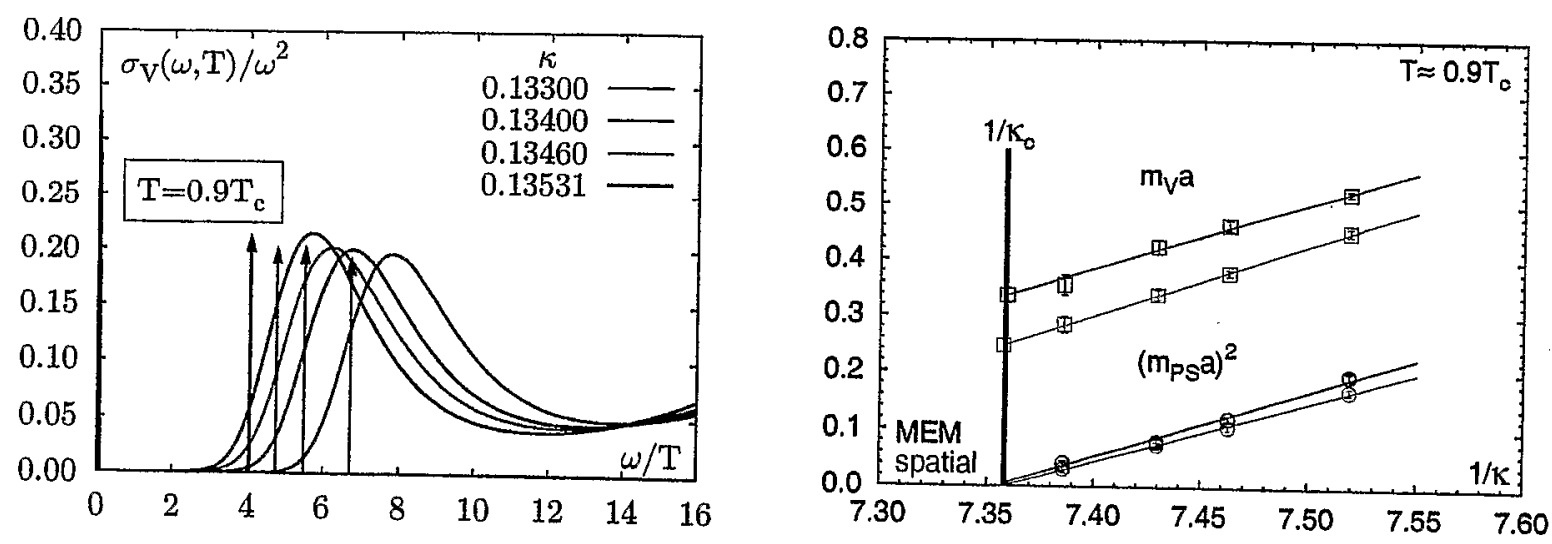

$T=0.9 T_{c}$

$\rho$ enhanced

over screening mass

finite size effect?

arrows denote screening mass position 
MEM results on $\sigma_{V}$ above $T_{c}$

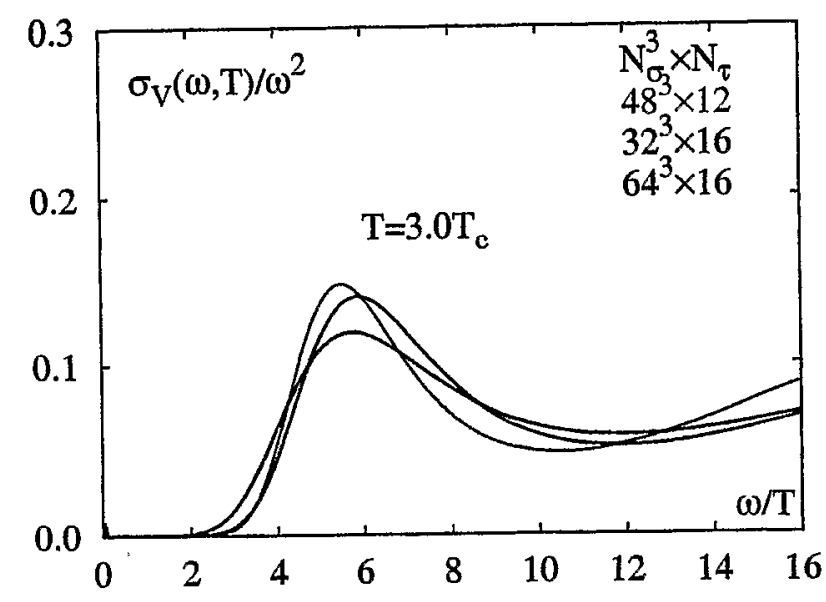

note

- different volumes: $N_{\sigma} / N_{\tau}=2,4$ at $N_{\tau}=16$

- different $a$ values: $N_{\tau}=12,16$ at $N_{\sigma} / N_{\tau}=4$

- no signal in low $\omega$ region, $\omega / T<2$

$$
\leadsto \text { sensitivity? }
$$

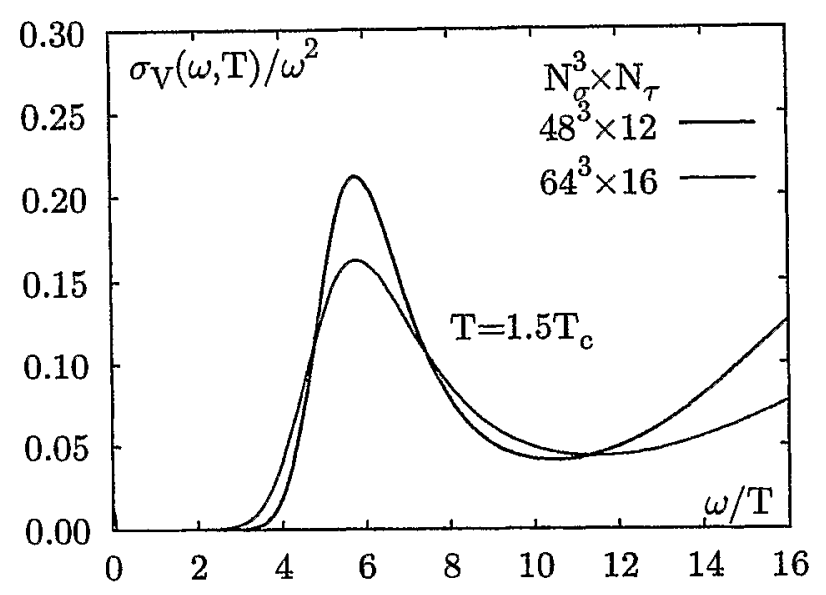

- clear, yet broad bump with maximum at

$$
\omega / T \simeq 6
$$

at temperatures $1.5 T_{c}$ and $3 T_{c}$

- around $\omega / T \simeq 5$ at $T=1.25 T_{c}$ (prelim.) 


\title{
Meson correlators in lattice QCD at finite temperature
}

\author{
Hideo Matsufuru* \\ High Energy Accelerator Research Organization (KEK), Tsukuba 305-0801, Japan \\ in collaboration with \\ Takashi Umeda (Yukawa Institute for Theoretical Physics, Kyoto Univ.) \\ Kouji Nomura (Department of Physics, Hiroshima Univ.) \\ Lattice $Q C D$ at Finite Temperature and Density \\ RIKEN BNL Research Center, 8-12 February 2004
}

\begin{abstract}
We study charmonium correlators in pseudoscalar and vector channels at finite temperature using lattice QCD simulation in the quenched approximation [1]. Anisotropic lattices are used in order to have sufficient numbers of degrees of freedom in the Euclidean temporal direction. We focus on the low energy structure of the spectral function, corresponding to the ground state in the hadron phase, by applying the smearing technique to enhance the contribution to the correlator from this region. We employ two analysis procedures: the maximum entropy method (MEM) for the extraction of the spectral function without assuming a specific form, to estimate the shape of the spectral function, and the standard $\chi^{2}$ fit analysis using typical forms in accordance with the result of MEM, for a more quantitative evaluation. A sophisticated version of the latter analysis, we employ the constrained curve fitting, which makes use of the prior knowledge about the system in question.

The numerical results are obtained on the quenched lattices of sizes $20^{3} \times N_{t}$, where $N_{t}=160$ $(T \simeq 0), 32\left(T \simeq 0.88 T_{c}\right), 30\left(0.93 T_{c}\right), 29\left(0.97 T_{c}\right), 27\left(1.04 T_{c}\right), 26\left(1.08 T_{c}\right), 24\left(1.17 T_{c}\right), 20\left(1.40 T_{c}\right)$, and $16\left(1.75 T_{c}\right)$. To verify the applicability of the procedures, we first analyze the smeared correlators as well as the point correlators at zero temperature. We find that by shortening the $t$-interval used for the analysis (a situation inevitable at $T>0$ ) the reliability of MEM for point correlators is lost, while it subsists for smeared correlators. Then the smeared correlators at finite temperatures. Below $T_{c}$, we find no significant mass shift and sizable width for the ground state peak. This result is inconsistent with naive potential model analyses using the static quark potential extracted from the Polyakov loop correlation. Above $T_{c}$, we find that the strong peak structure still persists at almost the same place as below $T_{c}$, but with a finite width of a few hundred $\mathrm{MeV}$. This result indicates that the correlators possess a nontrivial structure even in the deconfined phase. This is also consistent with the observation of $c-\bar{c}$ spatial correlation in the Coulomb gauge, which also indicated strong correlation between quark and antiquark exist up to $T \simeq 1.5 T_{c}$ [2].
\end{abstract}

\section{References}

[1] T. Umeda, K. Nomura and H. Matsufuru, hep-lat/0211003 (to appear in Eur. Phys. J. C)

[2] T. Umeda, R. Katayama, O. Miyamura and H. Matsufuru, Int. J. Mod. Phys. A 16 (2001) 2215.

"Email: hideo.matsufuru@kek.jp ; URL: http://www.rcnp.osaka-u.ac.jp/ matufuru/ 
Our approach

Properties of mesonic modes through the spectral function.

- Apply as many procedures as possible.

- Maximum entropy method (MEM)

- Fit analysis assuming presumable forms $(\leftarrow$ MEM) (constrained curve fitting)

- Semi-quantitative analysis.

- evaluation of mass shift, width

- Smearing - enhancement of low energy part

$\circ \leftarrow$ Problem of point correlators

$\circ \rightarrow$ Uncertainty from smearing functions

- Anisotropic lattice: finer temporal lattice spacing $a_{\tau}$ than $a_{\sigma}$

- Sufficient number of d.o.f. in temporal direction

- Toward dynamical simulations 


\section{Point correlators at $T=0$}

\section{Restriction of data points used in MEM analysis}
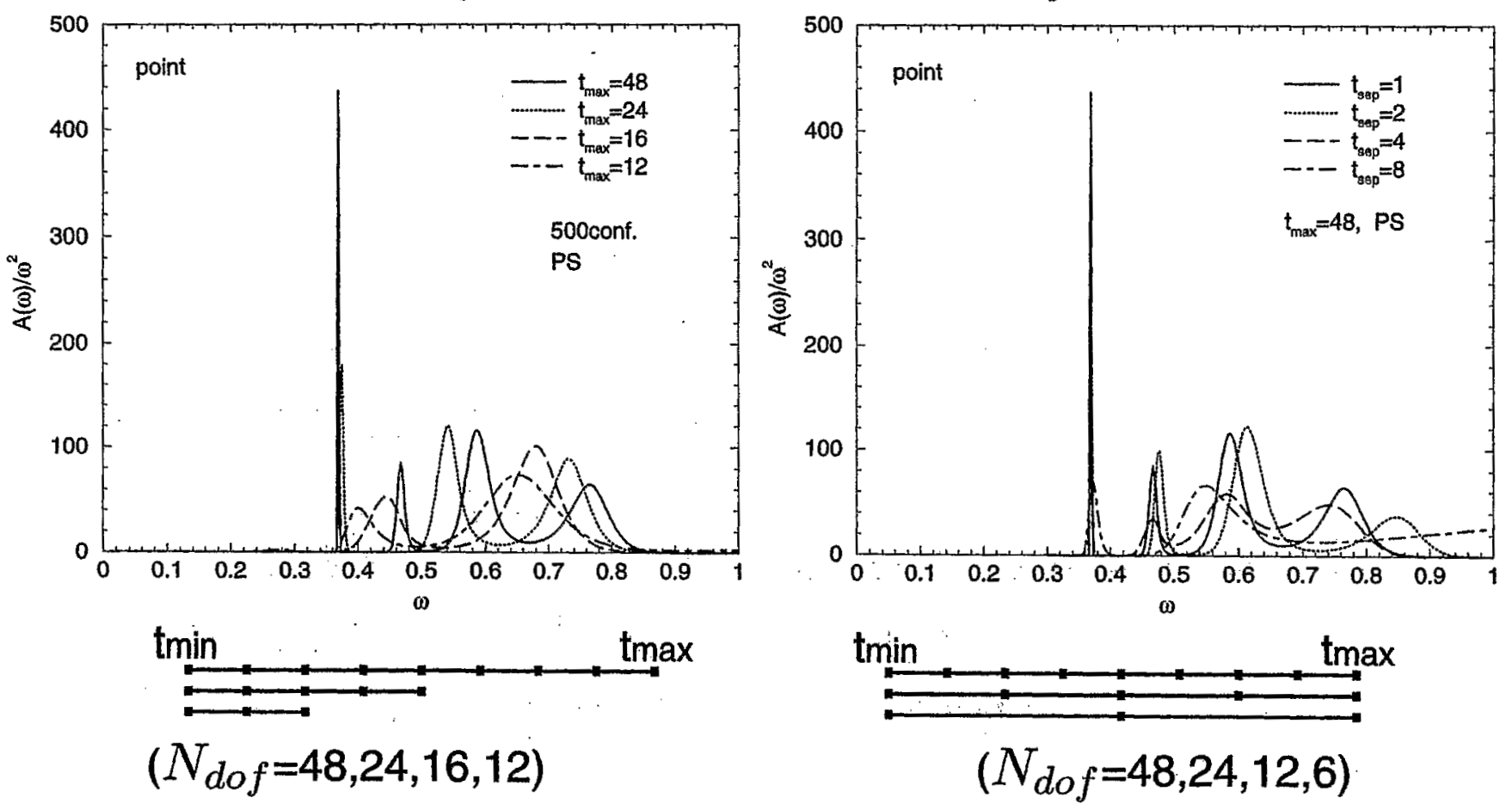

- $O(10)$ d.o.f. is sufficient

- $N_{t} a_{\tau} \sim 1 \mathrm{fm}$ is necessary 
$+$

Results below $T_{c}$ : Fit results

Fit results (constrained curve fitting).
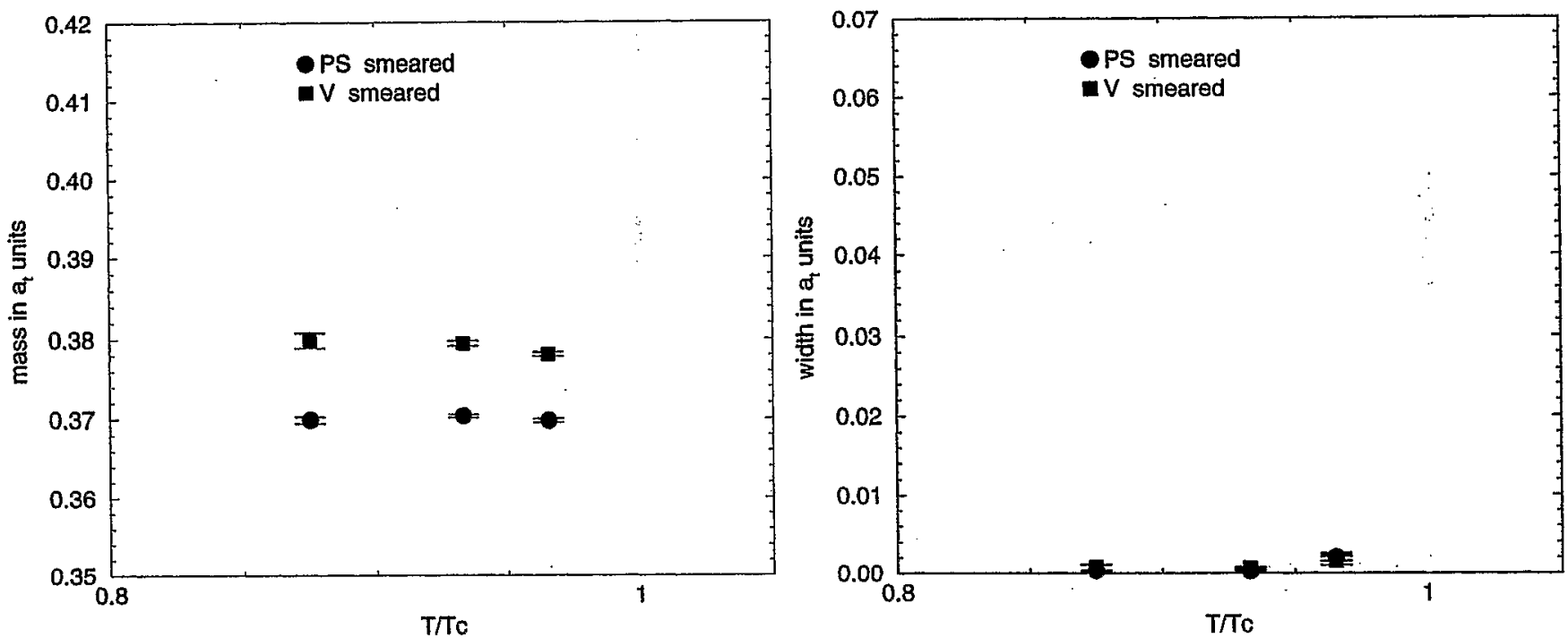

- No significant mass shift is observed.

- No sizable width is observed. 
Results above $T_{c}$ : MEM results $T \simeq 1.08-1.75$

MEM results ( $N_{t} \leq 20$ is less reliable).
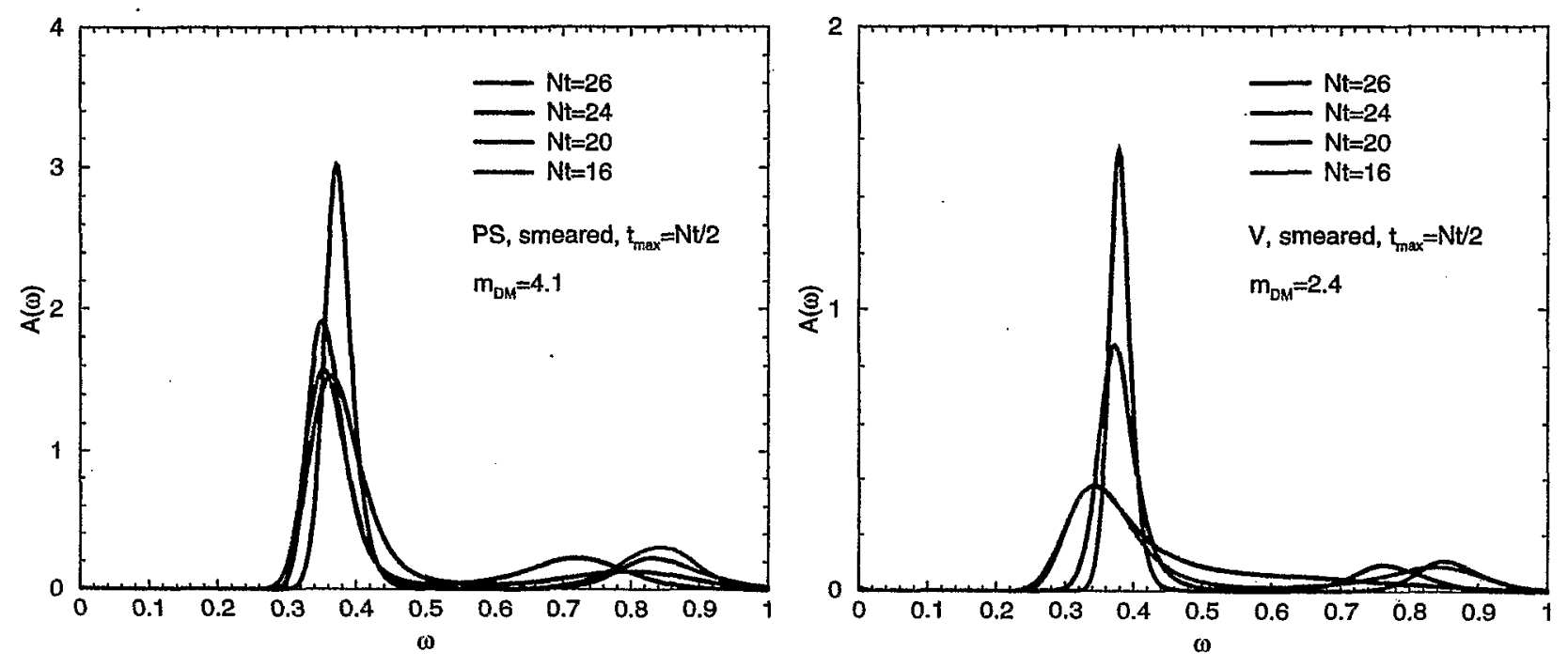

- Correlators change gradually as $T$ increases

- Small or no shift of peak position

- Vector channel shows large change at high $T$ 
At $T>T_{c}$ : Fit analysis at $T \simeq 1.08 T_{c}$

Result of standard $\chi^{2}$ fit analysis with BW+pole form
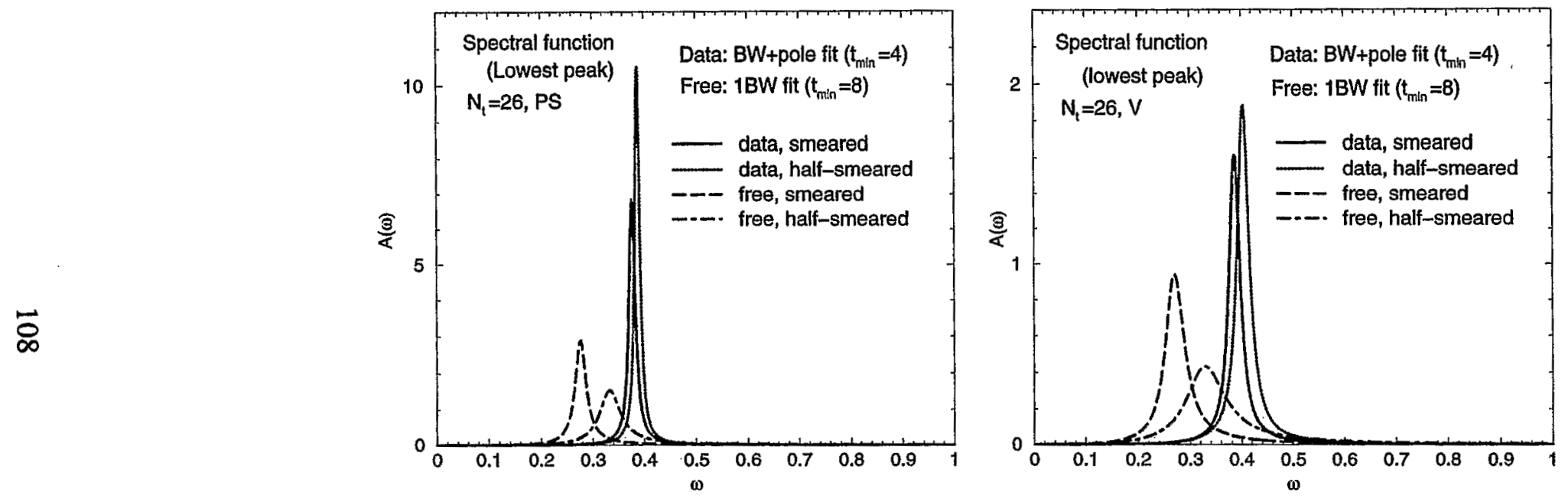

By changing smearing function,

- Free quark case:

peak shift more than the widths / widths become broader

- Numerical result:

peak does not shift beyond the widths / stable widths

$\Rightarrow$ We conclude peaks are physical (mesonic modes) 


\title{
Temporal and spatial hadron correlators in finite temperature $Q C D$
}

\author{
Sourendu Gupta (TIFR, Mumbai) \\ February 10, 2004
}

1. Screening correlators: departure from weak coupling theory?

2. Temporal correlators: transport coefficients and linear response theory.

- Bayesian methods- functional and parametrised

- Bayesian analysis of lattice data: yields small transport time scale

3. Some phenomenology 


\section{Screening masses}

Staggered quark screening masses agree with free field theory. Insignificant quark mass dependence for $m \leq T$. Overlap quarks at coarse lattice spacing give similiar results.

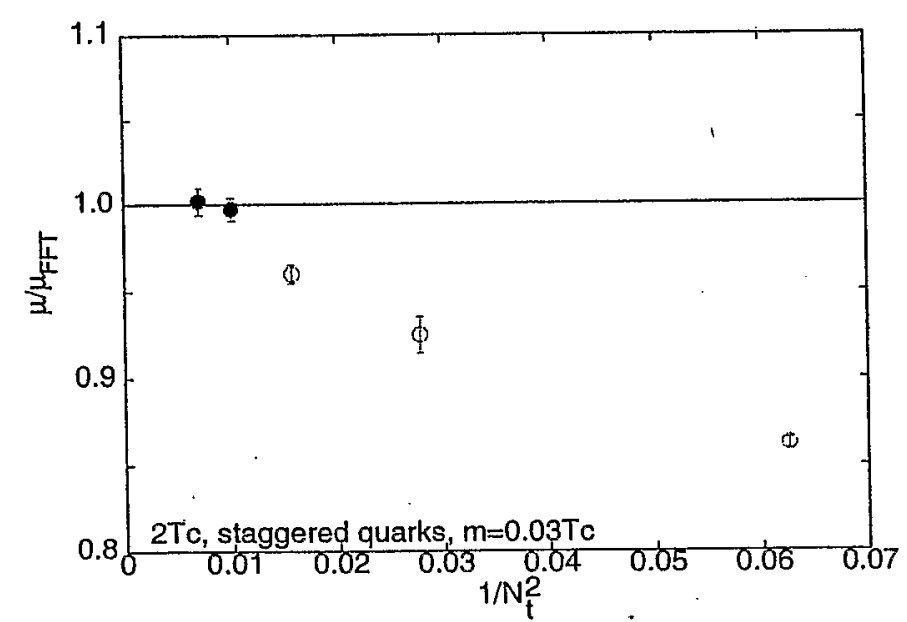

Gavai and SG, Phys. Rev., D 67 (2003) 034501

Laine and Vepsalainen, hep-ph/0311268 and Hansson and Zahed, Nucl. Phys., B 374 (1992) 277 


\section{Long distance physics}

Screening mass does not capture the full physics. Momentum space correlator shows that long distance physics is different from free field theory of quarks. There is also too much power in the long distance part of the correlator when compared to a single boson exchange.

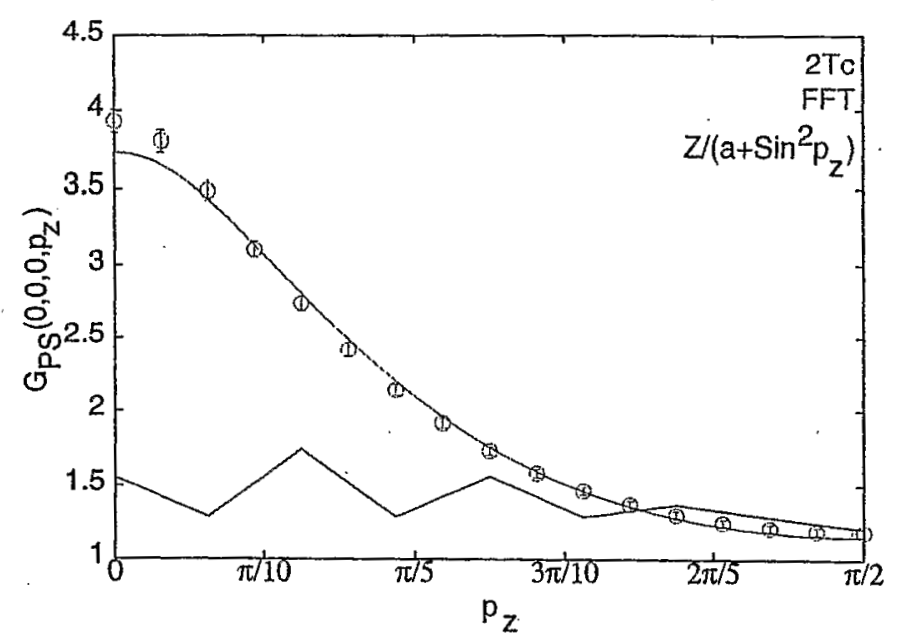




\section{Electrical conductivity: continuum limit}

Electrical conductivity depends only on the parameter $\gamma$. Obtain this by marginalising over the remaining parameters. SG, hep-lat/0301006

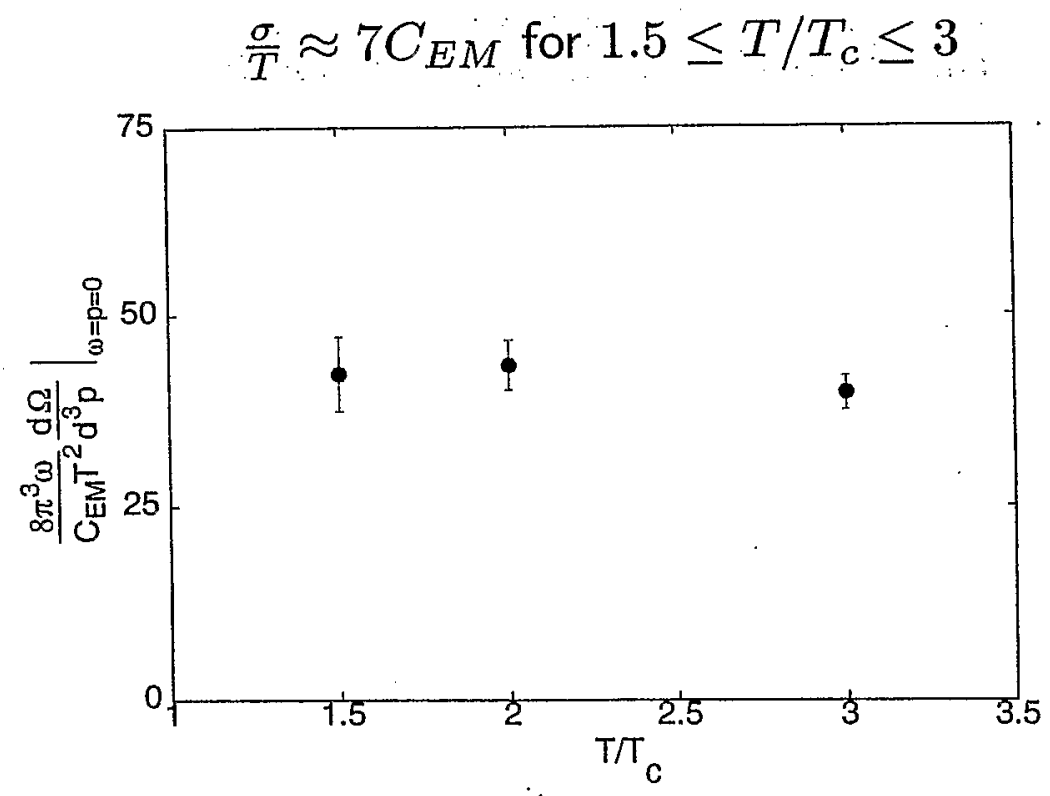




\section{Parametric behaviour}

$$
\Delta G\left(\omega_{n}, \mathbf{p} ; T\right)=\oint \frac{d \omega}{2 i \pi} \frac{\Delta \rho(\omega, \mathbf{p} ; T)}{\omega-\omega_{n}}
$$

dominated by poles closest to the origin. If these are poles of $\Delta \rho$ then

\section{Relaxation time}

$\vec{\omega}$

\section{Landau damping}

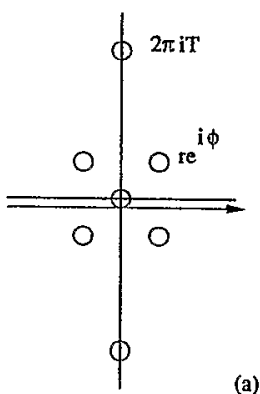

- $\left|\Re G\left(\omega_{n}\right)\right| \gg\left|\Im G\left(\omega_{n}\right)\right|$

- $\operatorname{sign} \Re G\left(\omega_{n}\right)=\operatorname{sign} \Im G\left(\omega_{n}\right)$

- $\Re G\left(\omega_{n}\right)<0$.

(*) $\quad\left|\Re G\left(\omega_{n}\right)\right| \gg\left|\Im G\left(\omega_{n}\right)\right|$

(*) $\quad$ sign $\Re G\left(\omega_{n}\right) \neq \operatorname{sign} \Im G\left(\omega_{n}\right)$

- $\Re G\left(\omega_{n}\right)>0$. 


\section{Lattice results}

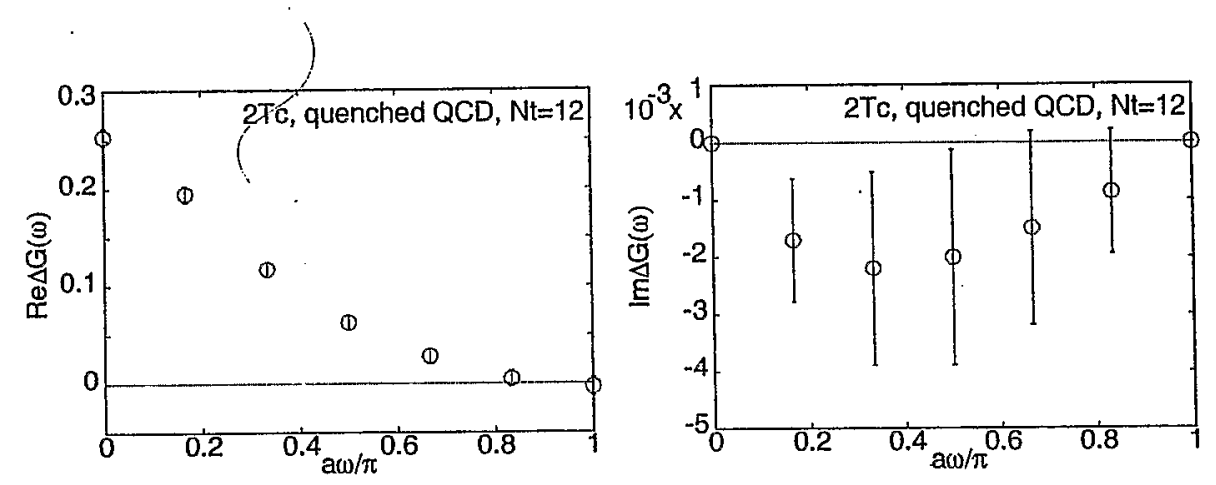

- Relaxation time approach ruled out. Landau damping possible.

- For $N_{t}=12$ fits possible with $|n| \leq 3$.

- Smaller lattices give qualitative results but a fit is not possible.

- $S_{4}$ poles at $\omega / 2 \pi T \approx 0.15$ and $\phi \approx 0.2$. 


\title{
LATTICE SIMULATIONS AT THE FERMI SURFACE
}

\author{
Simon Hands \\ Department of Physics, University of Wales Swansea, \\ Singleton Park, Swansea SA2 8PP, U.K.
}

\begin{abstract}
Controlled calculations of the properties of QCD with baryon chemical potential $\mu_{B} \sim O(1 \mathrm{GeV})$ remain an elusive dream; progress is possible, however, in simpler effective models which do not suffer from the fermion sign problem. In my talk I review results from the Gross-Neveu and NJL models in both $2+1$ and $3+1$ dimensions using both analytical and numerical methods. For $\mu$ greater than a critical value the model is chirally symmetric and has a Fermi surface with $k_{F} \simeq \mu$. I show how to calculate the particle interaction in medium due to scalar meson exchange to leading order in $N_{f}^{-1}$, where $N_{f}$ is the number of flavors, in the hard dense loop approach. This is then used to calculate the relation between $\mu$ and the Fermi momentum and velocity to $O\left(N_{f}^{-1}\right)$, using the theory of the Fermi liquid originally formulated by Landau. Next, simulation results (using a standard hybrid Monte Carlo algorithm) from a $32^{2} \times 48$ lattice for fermion and meson dispersion relations are presented, showing qualitative and in some cases quantitative agreement with analytic predictions. The most spectacular disagreement is in $\mathrm{NJL}_{3+1}$ where the fermion dispersion relation has an energy gap $\Delta$ of similar order of magnitude to that predicted in effective analytic descriptions and shows that the Fermi liquid is unstable with respect to the superfluid (aka color-superconducting) ground state. The simulations of $\mathrm{GN}_{2+1}$ show evidence for a massless pole in the vector meson channel resembling a collective mode known as zero sound. Finally, I make some remarks on the High Density Effective Theory recently formulated by Hong \& Hsu, and in particular discuss how it must be modified to reproduce Fermi liquid physics.
\end{abstract}

\section{References}

[1] SJH, J.B. Kogut, C.G. Strouthos \& T.N. Tran, Phys. Rev. D68 (2003) 016005.

[2] SJH, Phys. Rev. D69 (2004) 014020.

[3] SJH \& D.N. Walters, hep-lat/0401018 
What can we say at smaller densites $\mu \sim O(1 \mathrm{GeV})$ where weak coupling methods can't be trusted? Lattice $Q C D$ simulations can't help because the Euclidean path integral measure $\operatorname{det} M(\mu)$ is not positive definite.

In condensed matter theory there are two tractable limits:

\begin{tabular}{ccc}
\hline & Strong Coupling & Weak Coupling \\
\hline physical d.o.f's & tightly-bound bosons & weakly interacting fermions
\end{tabular}

$\begin{array}{ccr}\begin{array}{c}\text { superfluidity } \\ \text { mechanism } \\ \text { underlying }\end{array} & \text { Bose Einstein Condensation } & \text { BCS condensation } \\ \text { perturbative system } & \text { U(1) scalar field } & \text { Fermi liquid } \\ \text { QFT example } & \text { Two Color QCD } & \text { NJL model }\end{array}$

Both model OFT's can be studied with $\mu \neq 0$ using lattice simulations.

High- $T_{c}$ superconducting compounds, and perhaps QCD, are difficult problems because they belong to neither limit

\section{Numerical Results with $N_{f}=4$}
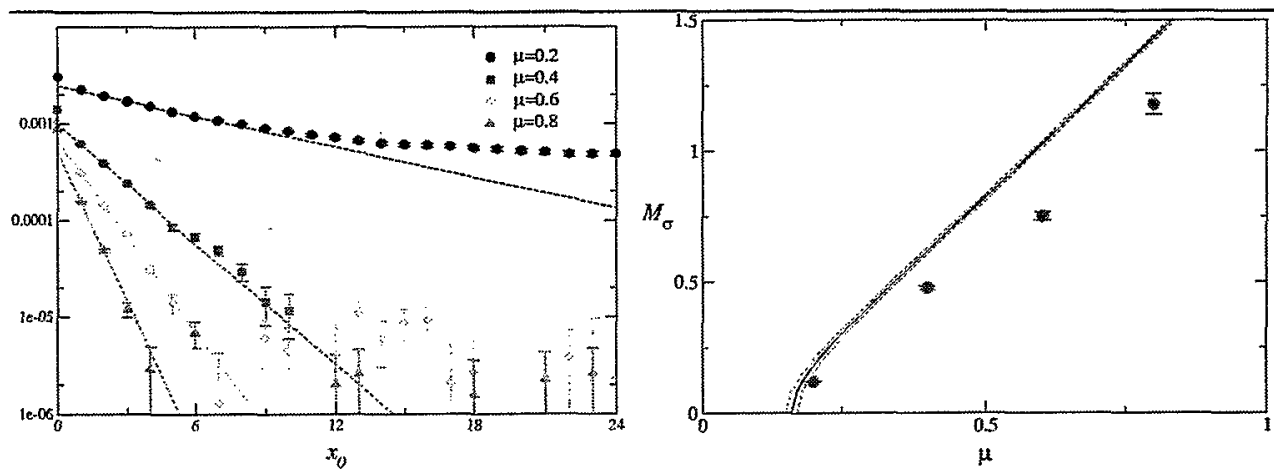

In quark matter by contrast the $\sigma$ exhibits a sharply-defined pole at $M_{\sigma}(\mu)$ consistent with $O\left(1 / N_{f}\right)$ corrections to the leading order result $M_{\sigma}=2 \sqrt{\mu\left(\mu-\mu_{c}\right)}$ with $\mu_{c} a \approx 0.16$ 

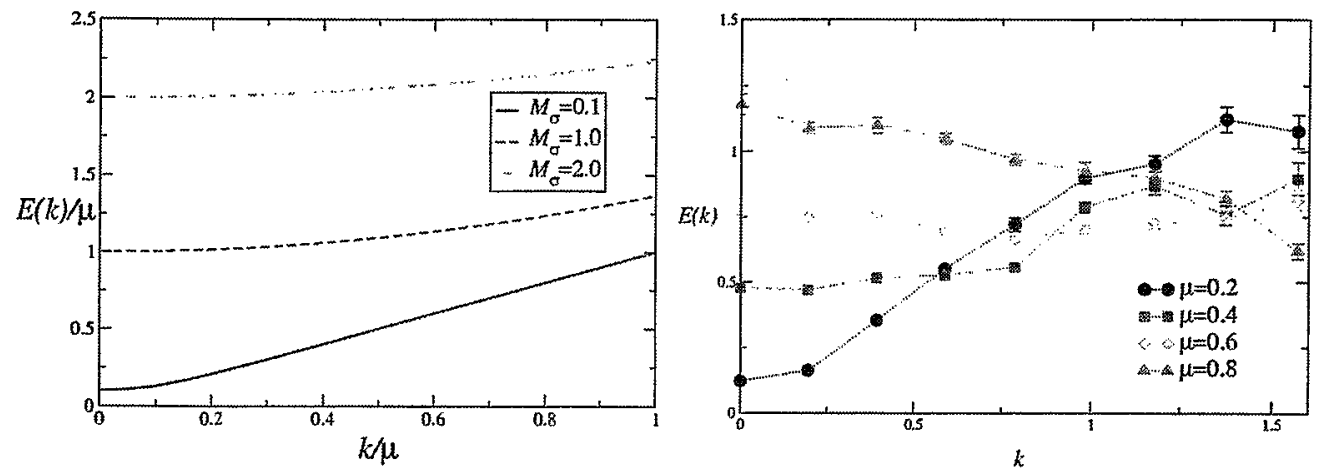

The $\sigma$ dispersion relation $E(|\vec{k}|)$ is also modified as $\mu \nearrow$ in qualitative agreement with the large- $N_{i}$ result.

Discretisation artifacts near the zone edge?

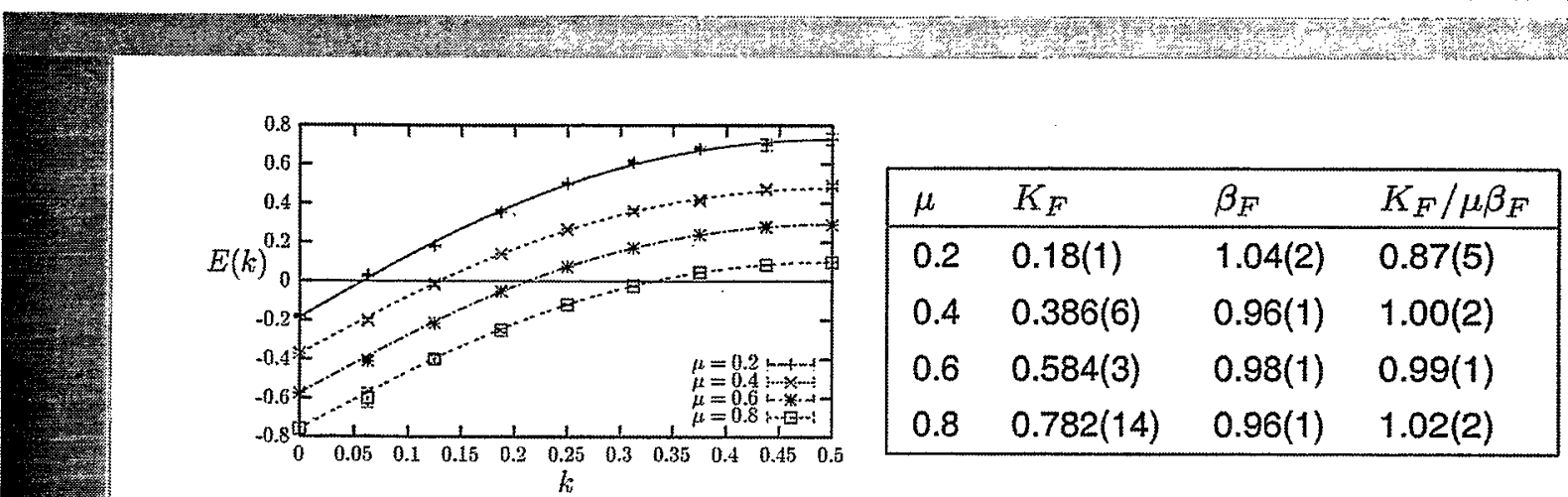

The fermion dispersion relation is fitted with

$$
E(|\vec{k}|)=-E_{0}+D \sinh ^{-1}(\sin |\vec{k}|)
$$

yielding the Fermi liquid parameters

$$
K_{F}=\frac{E_{0}}{D} ; \quad \beta_{F}=D \frac{\cosh E_{0}}{\cosh K_{F}}
$$

Results for GN model consistent with $\mathfrak{g}=2, N_{f}=4$ 


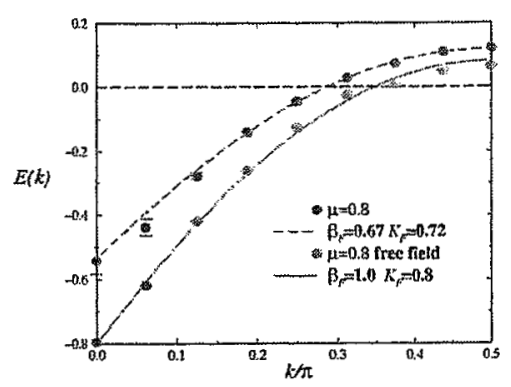

\begin{tabular}{|llll|}
\hline$\mu$ & $K_{F}$ & $\beta_{F}$ & $K_{F} / \mu \beta_{F}$ \\
\hline 0.80 & $0.720(3)$ & $0.670(3)$ & $1.34(1)$ \\
0.82 & $0.738(3)$ & $0.671(3)$ & $1.34(1)$ \\
0.84 & $0.773(3)$ & $0.686(3)$ & $1.34(1)$ \\
0.86 & $0.791(5)$ & $0.673(4)$ & $1.37(1)$ \\
0.88 & $0.811(5)$ & $0.628(4)$ & $1.47(1)$ \\
0.90 & $0.836(4)$ & $0.704(4)$ & $1.32(1)$ \\
\hline
\end{tabular}

[SJH, B.Lucini and S. Morrison, PRD65(2002)036004]

Simulations of $\mathrm{NJL}_{2+1}$, however, yeld Fermiliquid parameters inconsistent with $\mathfrak{g}=4, N_{f}=4$

Evidence for new physics due to phase coherent diquark pairing at Fermi surface?

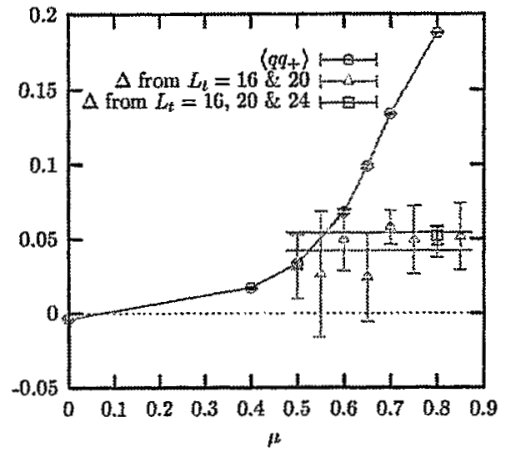

[S.JH and D.N. Walters, hep-lat/0401018]

Finally, simulations of $N L_{3+1}$, reveal a genuine gap at the Fermi surface consistent with an orthodox BCS mechanism as a result of diquark pair condensation

The ratio $\Delta / \Sigma_{0} \approx 0.15 \Rightarrow \Delta \approx 60 \mathrm{MeV}$

This result is comparable with self-consistent approaches 


\section{Meson Correlation Functions}

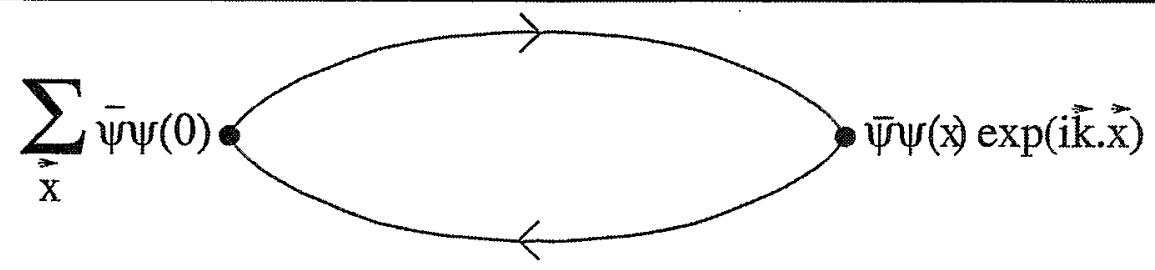

For $\vec{k} \neq 0$ can always excite a particle-hole pair with almost zero energy $\Rightarrow$ algebraic decay of correlation functions

$|\vec{k}| \ll \mu$

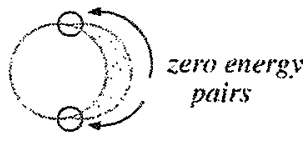

$\Rightarrow C \sim \frac{1}{x_{0}^{2}}$

$|\vec{k}|=2 \mu$

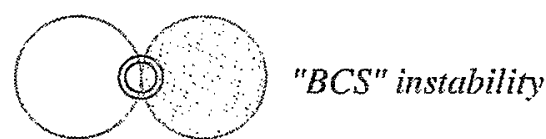

$\Rightarrow C \sim \frac{1}{x_{0}^{3 / 2}}$

$|\vec{k}|>2 \mu$

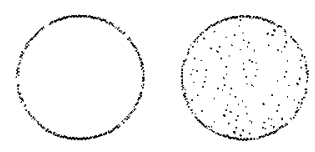

$\Rightarrow C \sim \frac{e^{-(|\vec{k}|-2 \mu) x_{0}}}{x_{0}^{3 / 2}}$

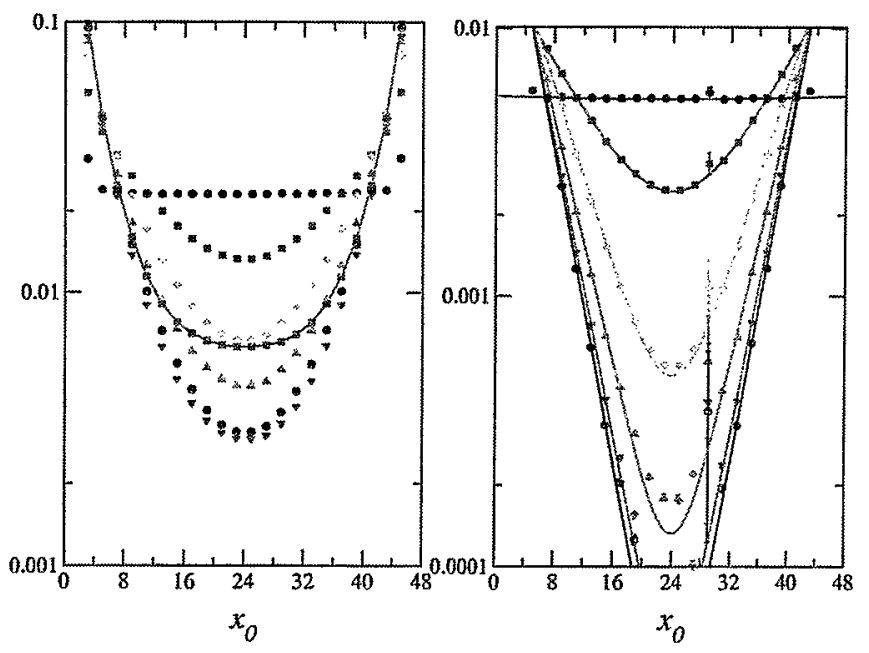

In the spin-1 channel, $C_{\gamma_{\perp}}$ also looks algebraic, but $C_{\gamma_{\|}}$ shows evidence of exponential decay. 


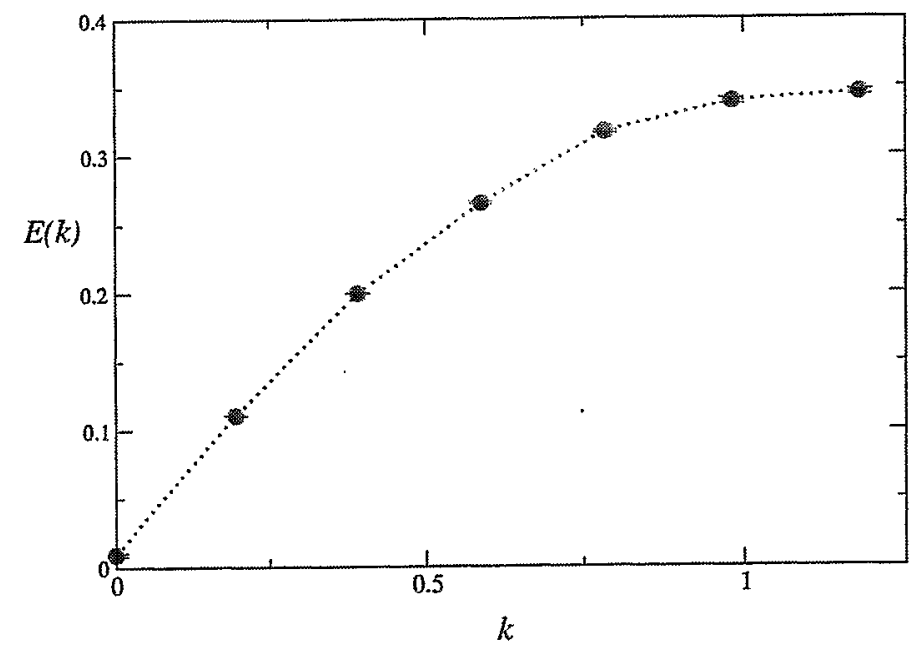

Dispersion relation $E(|\vec{k}|)$ extracted from $C_{\gamma_{\|}}$at $\mu a=0.6$ A massless vector excitation?

\section{Sounds Unfamiliar?}

Light vector states in medium are of of great interest:

Brown-Rho scaling, vector condensation... In the Fermi liquid framework a possible explanation is a collective excitation thought to become important as $T \rightarrow 0$ : Zero Sound

Ordinary FIRST sound is a breathing mode

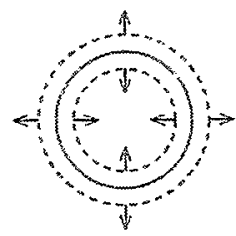
of the Fermi surface: velocity $\beta_{1} \simeq \frac{1}{\sqrt{ } 2} \frac{k_{F}}{\mu}$

ZERO sound is a propagating distortion

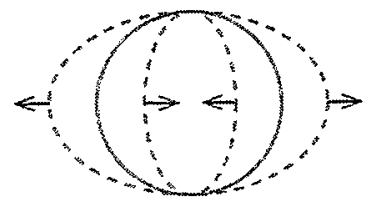
of the Fermi surface: velocity $\beta_{0}$ must be determined self-consistently 


\title{
Order from disorder in lattice QCD with baryons
}

\author{
Barak Bringoltz \\ School of Physics and Astronomy, Raymond and Beverly Sackler Faculty of Exact \\ Sciences, Tel Aviv University, 69978 Tel Aviv, Israel
}

Email: barak@viki.tau.ac.il — http://viki.tau.ac.il/ barak

We determine the patterns of spontaneous symmetry breaking in strong-coupling lattice QCD with baryons. Our starting point is the effective Hamiltonian for color singlet objects, which looks in lowest order like an antiferromagnet, and which describes meson physics with a fixed baryon background. The symmetry of the Hamiltonian depends on the underlying fermion formulation. For naive nearest-neighbor (NN) fermions the symmetry is $U\left(4 N_{f}\right)$. We transform the Hamiltonian to a path integral of a non-linear sigma model and use large- $N_{c}$ techniques to extract the ground state and excitation spectrum. We find that at lowest order the classical ground state is locally degenerate. Quantum fluctuations of $O\left(1 / N_{c}\right)$ remove this degeneracy and choose a ground state that breaks the $U\left(4 N_{f}\right)$ symmetry. This is a manifestation of the condensed-matter phenomenon called "order from disorder". We construct the excitation spectrum along the lines of order from disorder, and find that this NN theory gives rise to two kinds of Goldstone bosons: type I Goldstone bosons that exists also at zero density and type II Goldstone bosons whose energy is proportional to $|\vec{k}|^{2}$. The type II bosons emerge only when $O\left(1 / N_{c}\right)$ fluctuations are taken into account.

When we use a next-nearest-neighbor fermion kernel for the underlying theory, the effective Hamiltonian acquires extra terms that break the symmetry to $U\left(N_{f}\right) \times$ $U\left(N_{f}\right)$. Treating these terms as a perturbation of the NN ground state, we find that both chiral symmetry and discrete lattice rotations are spontaneously broken. This is accompanied by interesting types of fluctuations, that are admixtures of type I and type II Goldstone bosons, and that have anisotropic dispersion relations.

[1] B. Bringoltz, and B. Svetitsky, Phys. Rev. D 69, 014502 (2004) [hep-lat/0310032].

[2] B. Bringoltz, hep-lat/0308018. To appear in Phys. Rev. D.

[3] B. Bringoltz and B. Svetitsky, Phys. Rev. D 68, 034501 (2003) [hep-lat/0211018]. 
$H_{\mathrm{eff}}^{(2)}$-the Heisenberg Anti-Ferromagnet

$$
H_{\mathrm{eff}}^{(2)}=\sum_{\substack{\mathbf{n} \mu \eta \\ l=1,2}} J_{l}\left(s_{\eta}^{\mu}\right)^{l+1} Q_{\mathbf{n}}^{\eta} Q_{\mathbf{n}+l \hat{\mu}}^{\eta} .
$$

- $J_{1,2}$ depend on the fermion kernel and obey $J_{2} \ll J_{1}$.

- $Q_{\mathrm{n}}^{\eta}$ are fermion bilinears:

$$
Q_{\mathbf{n}}^{\eta}=\sum_{a=1}^{N_{c}} \sum_{\alpha, \beta=1}^{4 N_{f}} \Psi_{\mathbf{n}}^{\dagger \alpha a} M_{\alpha \beta}^{\eta} \Psi_{\beta a \mathbf{n}}-\frac{N_{c}}{2} \operatorname{Tr} M^{\eta} .
$$

- The set $M^{\eta}=\Gamma^{A} \otimes \lambda^{f}$ are the generators of $U\left(4 N_{f}\right)$, acting on Dirac-flavor indices.

$\Downarrow$

The "spin" operators $\boldsymbol{Q}_{\mathbf{n}}^{\eta}$ generate a $U\left(4 N_{f}\right)$ algebra.

- $s_{\eta}^{\mu}$ is a sign factor. Calculation of $s_{\eta}^{\mu}$ gives

$-\underline{l=1(\mathrm{NN}):} H_{\mathrm{eff}}^{(2)}-U\left(4 N_{f}\right)$ NN Heisenberg AFM.

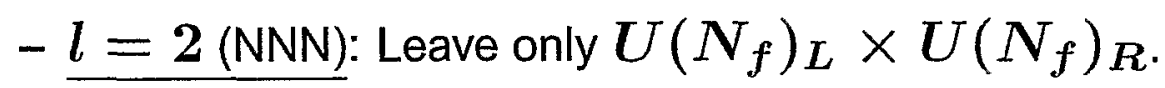




\section{Spin coherent states ( $\sigma$ model) representation}

On site $\mathbf{n}$, with baryon number $B_{\mathbf{n}}=m-2 N_{f}$, define "spin" coherent states

$|q\rangle \equiv \exp \left(\sum_{\alpha=1}^{m} \sum_{\beta=m+1}^{4 N_{f}}\left(q_{\beta}^{* \alpha} \Psi^{\dagger \beta a} \Psi_{\alpha a}-q_{\beta}^{\alpha} \Psi^{\dagger \alpha a} \Psi_{\beta a}\right)\right)|0\rangle$.

$|0\rangle$ - highest weight state in

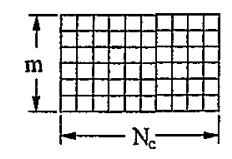

Write a path integral for the partition function $\rightarrow$ NLSM:

- $\sigma$ fields are $4 N_{f} \times 4 N_{f}$ matrices that are both unitary and hermitian:

$$
\begin{aligned}
\sigma_{\mathbf{n}}=U_{\mathbf{n}} \Lambda U_{\mathbf{n}}^{\dagger} & =\left\langle q\left|\vec{Q}_{\mathbf{n}} \cdot \vec{M}\right| q\right\rangle \text { with } U_{\mathbf{n}} \in U\left(4 N_{f}\right), \\
\Lambda & =\left(\begin{array}{cc}
1_{m} & 0 \\
0 & -1_{4 N_{f}-m}
\end{array}\right) .
\end{aligned}
$$

This means that,

$$
\sigma_{n} \in U\left(4 N_{f}\right) /\left[U\left(m_{n}\right) \times U\left(4 N_{f}-m_{n}\right)\right] .
$$


- The action is

$$
S=\frac{\mathbf{N}_{\mathbf{c}}}{\mathbf{2}} \int_{0}^{\beta} d \tau \operatorname{Tr}\left(\sum_{\mathbf{n}}-\Lambda U_{\mathbf{n}}^{\dagger} \partial_{\tau} U_{\mathbf{n}}+\frac{N_{c}}{4} \mathcal{H}_{\mathrm{eff}}^{(2)}\right)
$$

- The $\mathrm{NN}+\mathrm{NNN}$ interactions are

$$
\mathcal{H}_{\mathrm{eff}}^{(2)}=\sum_{\mathbf{n}, \hat{\mu}} J_{1}\left[\sigma_{\mathbf{n}} \sigma_{\mathbf{n}+\hat{\mu}}\right]+J_{2}\left[\sigma_{\mathbf{n}} \alpha_{\mu} \sigma_{\mathbf{n}+2 \hat{\mu}} \alpha_{\mu}\right] .
$$


Vl. Large $N_{c}$ and classical vacuum

Restrict to uniform $\boldsymbol{B}_{\mathbf{n}}=B \geq \mathbf{0}, \cdots \cdots \boxplus \boxplus \boxplus \boxplus \cdots$

Restrict to $J_{2}=0$, so symmetry is $U\left(4 N_{f}\right)$.

At large $N_{c}$ - minimize $\mathcal{H}_{\text {eff }}^{(2)}$ - minimize first the one link problem:

$$
E_{\text {link }}=J_{1} \operatorname{Tr} \sigma_{1} \sigma_{2}
$$

So what happens for $B>0$ ?

Minimum: any rotation of

$\sigma_{1}=\Lambda=\left(\begin{array}{cc}1_{m} & 0 \\ 0 & -1_{4 N_{f}-m}\end{array}\right), \sigma_{2}=\left(\begin{array}{c|c}\sigma_{m} & 0 \\ \hline 0 & 1_{4 N_{f}-m}\end{array}\right)$

$\sigma_{m} \equiv u_{m}\left(\begin{array}{cc}1_{2 m-4 N_{f}} & 0 \\ 0 & -1_{4 N_{f}-m}\end{array}\right) u_{m}^{\dagger}, \quad u_{m} \in U(m)$

- g.s. of link is pictorially

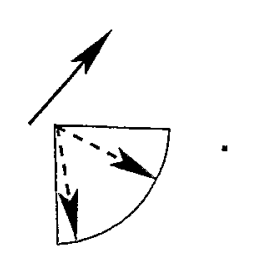




\section{IIX. Quantum fluctuations remove "zero modes"}

Write $\sigma_{\mathbf{n}}$ function of manifold coordinates.

- outside degenerate submanifold.

- within degenerate submanifold - zero modes.

$O\left(1 / N_{c}\right)$ - zero modes get nonzero self-energy, and have

- Type I GBs / AFM spin waves:

$$
\epsilon_{\mathbf{k}}^{I} \sim J_{1}|\mathbf{k}|, \quad n_{I}=2\left(4 N_{f}-m\right)^{2} .
$$

- Type II GBs / FM magnons (previously zero modes):

$$
\epsilon_{\mathbf{k}}^{I I} \sim \frac{J_{1}}{N_{c}}|\mathbf{k}|^{2}, \quad n_{I I}=2\left(4 N_{f}-m\right)\left(2 m-4 N_{f}\right) .
$$

Nielsen-Chadha counting rule is obeyed:

$$
n_{I}+2 n_{I I}=n_{G} .
$$

$$
\begin{aligned}
& U\left(4 N_{f}\right) \rightarrow \\
& U\left(2 m-4 N_{f}\right) \times U\left(4 N_{f}-m\right) \times U\left(4 N_{f}-m\right)
\end{aligned}
$$


IX. NNN interactions, $0<J_{2} \ll J_{1}$

$U\left(4 N_{f}\right) \rightarrow U\left(N_{f}\right)_{L} \times U\left(N_{f}\right)_{R}$, Vacuum alignment.

\begin{tabular}{cccc}
$N_{f}$ & $|B|$ & Unbroken symmetry & Broken charges \\
\hline 0 & - & 1
\end{tabular}

$\begin{array}{llll}1 & 1 & - & 1\end{array}$

\begin{tabular}{lll}
2 & $U(1)_{A}$ & 0 \\
\hline 0 & $S U(2)_{V}$ & 4
\end{tabular}

\begin{tabular}{cccc} 
& 1 & $U(1)_{I_{3}}$ & 6 \\
& 2 & $S U(2)_{V}$ & 4 \\
& 3 & $U(1)_{I_{3}}$ & 6 \\
& 4 & $S U(2)_{L} \times S U(2)_{R} \times U(1)_{A}$ & 0 \\
\hline \multirow{3}{*}{3} & $S U(3)_{V}$ & 9 \\
& 1 & $U(1)_{Y} \times S U(2)_{V}$ & 13 \\
& 2 & $U(1)_{Y}$ & 16 \\
& 3 & $S U(3)_{V}$ & 9 \\
& 4 & $U(1)_{I_{3}} \times U(1)_{Y}$ & 15 \\
& 5 & $U(1)_{I_{3}} \times U(1)_{Y} \times U(1)_{A}$ & 14 \\
6 & $S U(3)_{L} \times S U(3)_{R} \times U(1)_{A}$ & 0 \\
\hline
\end{tabular}

Some type II bosons get a mass $M \sim J_{2}$.

Some type II $\rightarrow$ type I, with $\epsilon_{\mathbf{k}} \sim \sqrt{\frac{J_{2} J_{1}}{N_{c}}} \sqrt{c_{z}^{2} k_{z}^{2}+c_{\perp}^{2} k_{\perp}^{2}}$. 
Feb.2004, finite $T$ lattice workshop

The Quark-Gluon Plasma at strong (QCD) and very strong (CFT) coupling

Edward Shuryak*, Stony Brook

- Good news: Large pressure at $T>T_{c}$ due to QGP is observed at RHIC. Furthermore hydrodynamics works well and lattice EoS results are quantiatively confirmed $\Rightarrow>T_{c} \approx$ $170 \mathrm{MeV}$, latent heat $\approx .7-.8 \mathrm{GeV} / \mathrm{fm}^{3}$.

- Moreover, contrary to expectations QGP at RHIC $\neq$ weakly interacting quasiparticle gas: its transport properties are such that QGP as seen at RHIC is the most perfect liquid known (viscosity/entropy $\eta / s \sim .1$ is much less than for e.g. water!)

*+G.Brown,D.Teaney,I.Zahed... 
- QGP at RHIC $\left(T=(1-3) T_{c}\right)$ is in a strongly interacting regime. What that means? We start to understand, with much help from two other (recently discovered) examples

- Trapped ultracold atoms at scattering length $a \rightarrow \infty$
- $\mathcal{N}=4$ SUSY $Y M=C F T$ at strong coupling $g^{2} N_{c}>>1$ (AdS/CFT duality $<=>$ string theory )

- hundreds of colored $q g, g g$ bound states on top of "old" $\bar{q} q$ and glueballs contribute to pressure.

- The zero binding lines inside the QGP phase are associated with large cross sections, maybe associated with the observed viscosity 


\section{Bound states of quasiparticles above $T_{c}$}

\section{ES and I.Zahed,hep-ph/0307267}
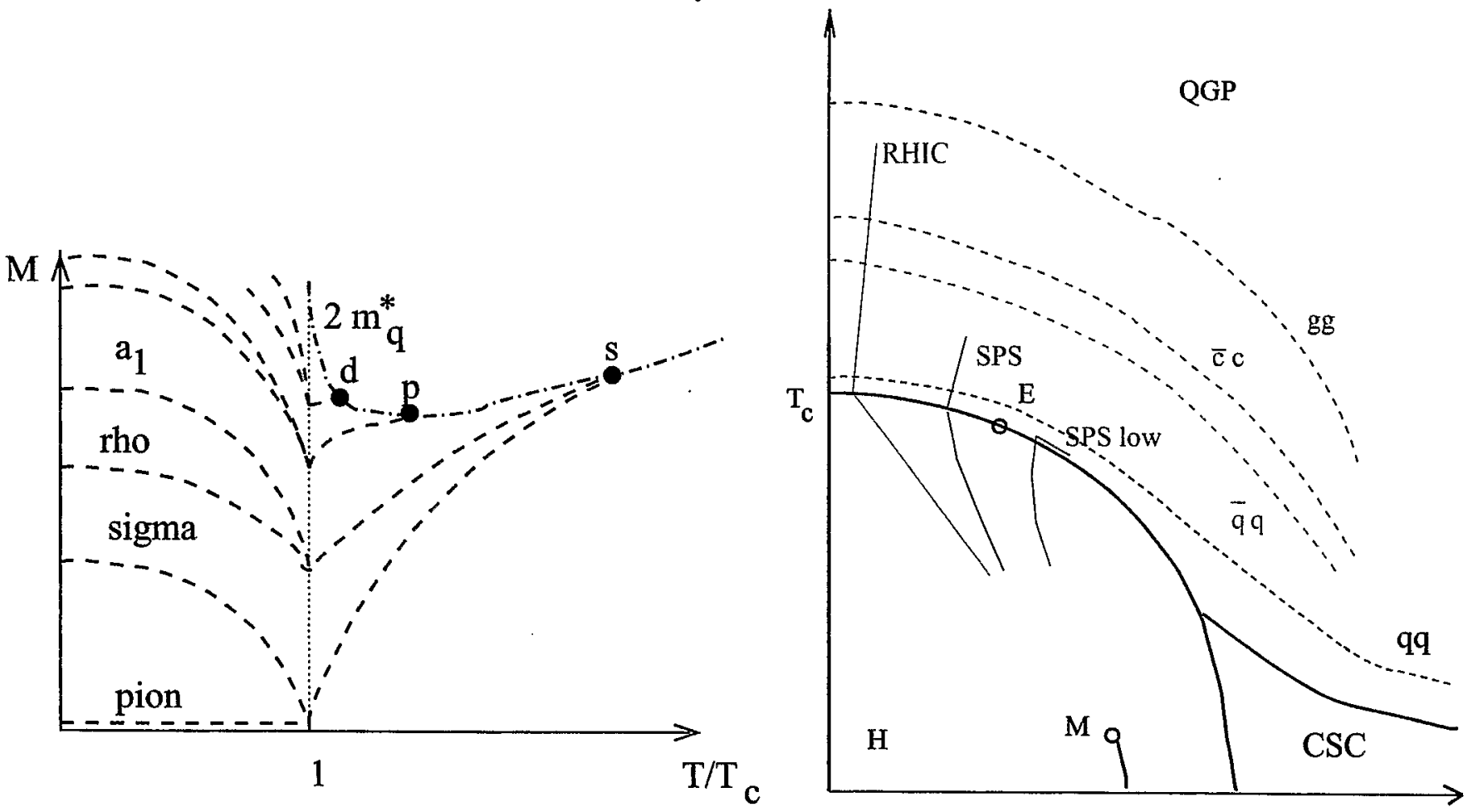

- Zero binding lines for various $\bar{q} q$ states (estimated for P-wave $T \approx 1.2 T c$, for S-wave at $1.5 T c$, charmonium at $1.6 \mathrm{Tc}$ from some model potential). 


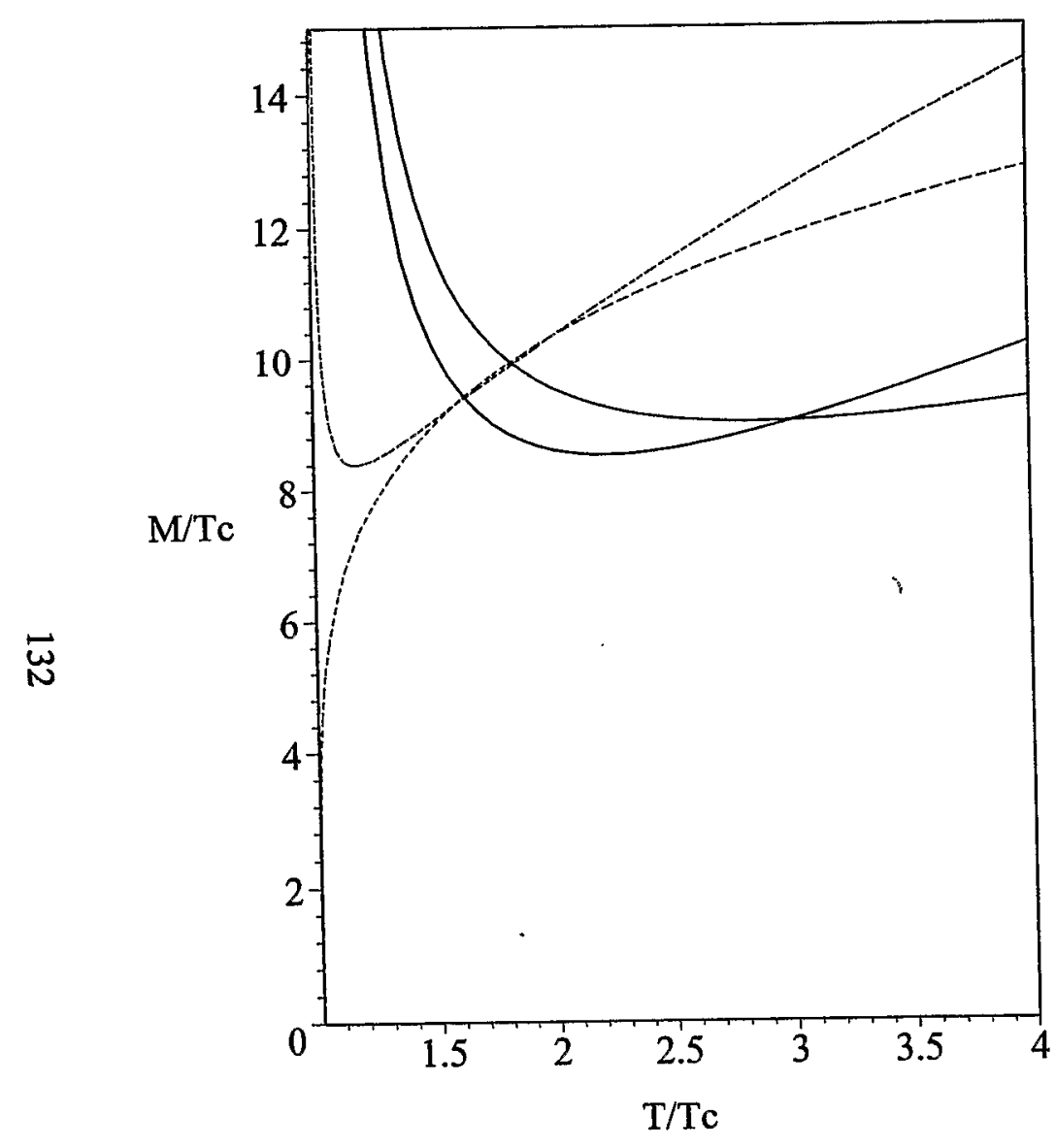

$2 M_{q}(T), 2 M_{g}(T)$ fitted to (Karsch et al) quasiparticle masses, as well as example of "old" $M_{\pi}(T)$ and "new" octet $M_{g g}^{8}(T)$

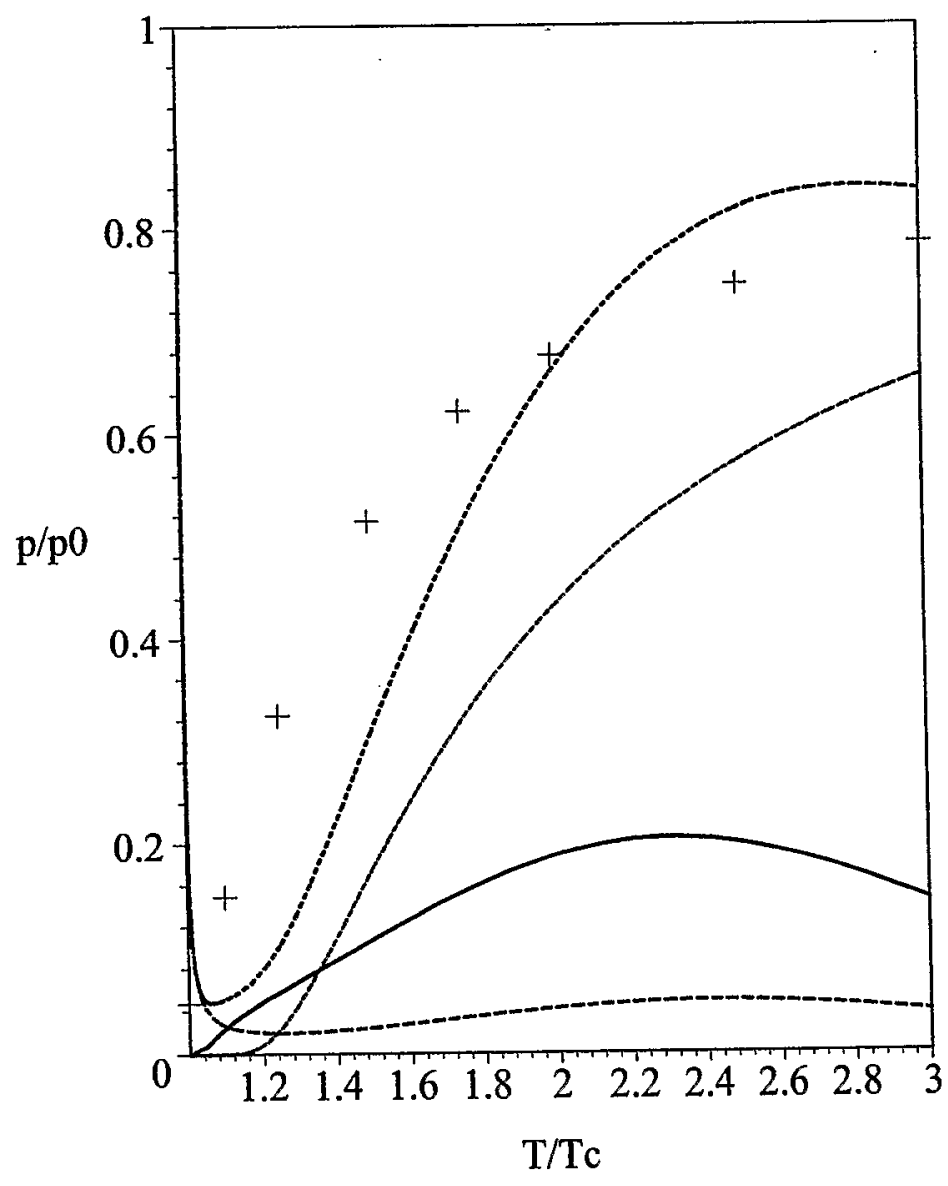

The QGP pressure: crosses are lattice thermodynamics for $N_{f}=$ 2 (Beilefeld,2000), the lines represent the contributions of $q+g$ quasiparticles, "mesons" $\pi-\rho \ldots$, colored exotics $\left(g g_{8}, q g_{3}\right)$ and total (the upper curve). 


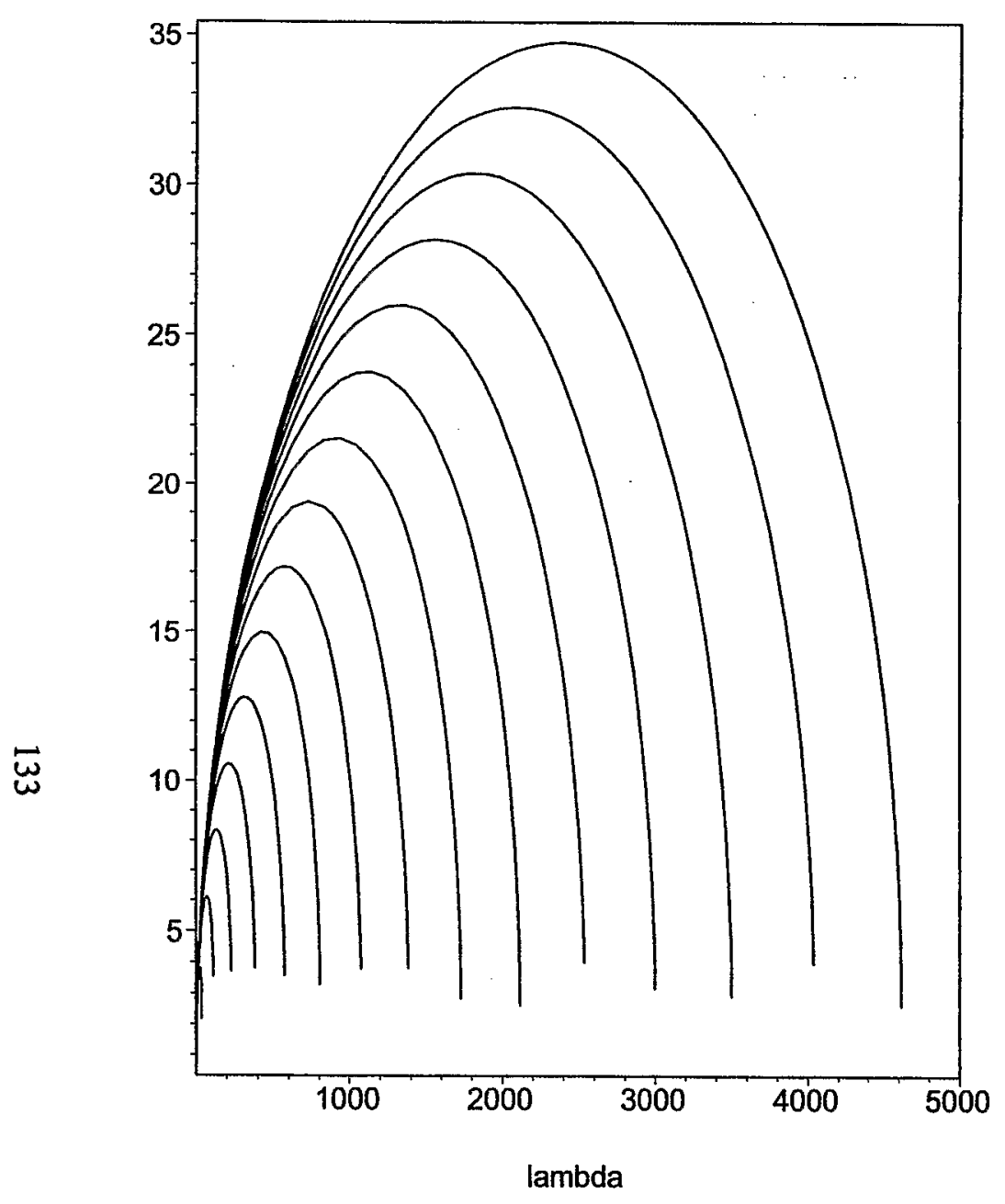

CoulombV $=-\frac{C}{r}$

$E_{n l} / M=$

$\left[1+\left(\frac{C}{n+1 / 2+\sqrt{(l+1 / 2)^{2}-C^{2}}}\right)^{2}\right]^{-1 / 2}$

Small C - nonrelat. atoms, Balmer series... New regime at large $C \sim$ $\lambda^{1 / 2}>1$ : families of relativistic deeply bound states, $E \sim \lambda^{0} T(n+$ $\left.1 / 2+\sqrt{(l+1 / 2)^{2}-C^{2}}\right)$ with large orbital momentum balancing the supercritical Coulomb 


\section{Summary}
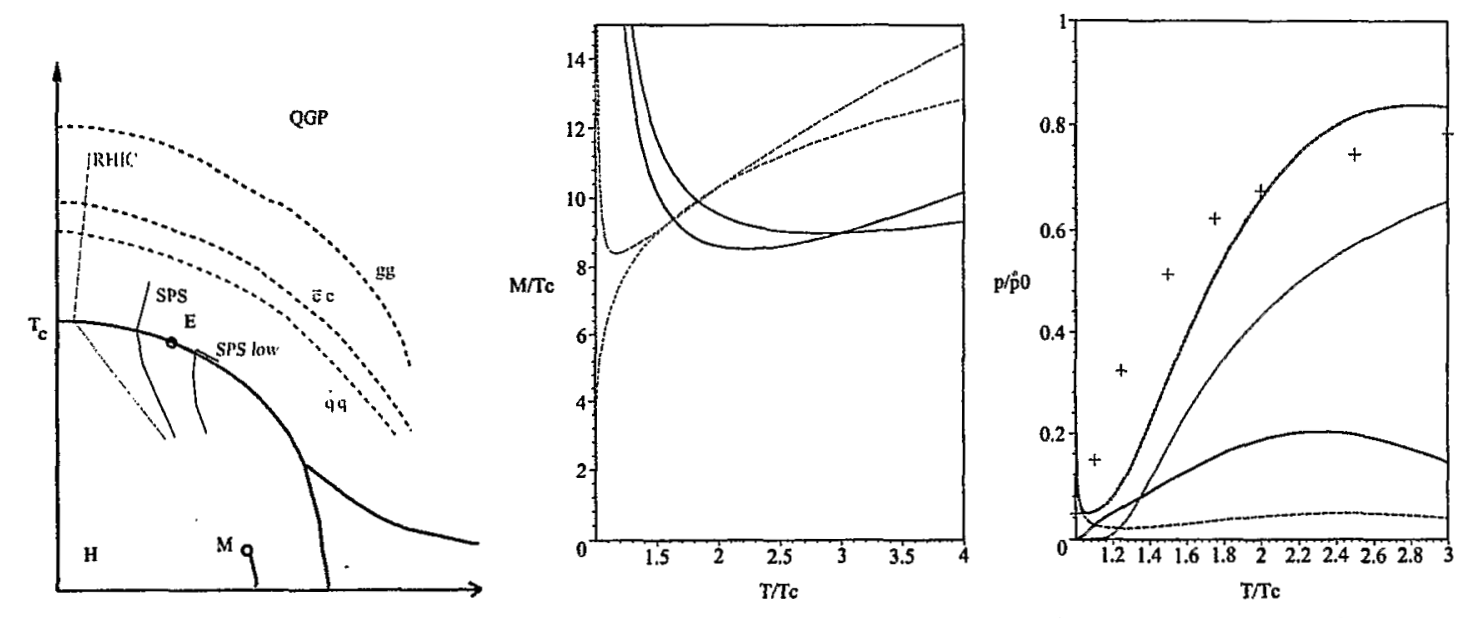

- QGP at RHIC does have large pressure predicted by the lattice, but we only start to understand why

- unexpectedly QGP is a surprisingly good fluid, with $\eta / s \sim 1 / 10$, smaller than ever seen before. This seem to be in conflict with $p Q C D$ (weak coupling) but suggests a strong coupling regime

- Connection to string theory via Ads/CFT correspondence by Maldacena provide good EoS and viscosity. Duality was recently extended to more QCDlike theories, can also be partly explained by ladder resummations...

- Not only known hadrons do not melt at $T>T_{c}$, but hundreds of colored exotic bound states of $q q, q g, g g$ also appear. All of them melt at some zero binding lines which separate "pure QGP".

- The exotic states generate about $1 / 2$ pressure at RHIC. Small mean free path is maybe due to strong (unitarity limited) rescattering e.g. $\bar{q} q<=>$ meson Amazingly similar to trapped $L i^{6}$ atoms. with "only" 19 orders difference in the $T$ scale 


\title{
Renormalized Polyakov Loops and Matrix Models
}

\author{
Jonathan T. Lenaghan \\ University of Virginia
}

Work done in collaboration with Adrian Dumitru, Yoshitaka Hatta, Kostas Orginos and Robert Pisarski

$$
\text { hep-th/0311223 }
$$

We discuss how to extract renormalized from bare Polyakov loops at nonzero temperature. The values of renormalized loops in the four lowest representations of $\mathrm{SU}(3)$ were measured numerically on small, coarse lattices. We find approximate agreement with a large $N$ expansion, where factorization implies that the expectation values of loops in adjoint and higher representations are just powers of fundamental and antifundamental loops. The values of the renormalized triplet loop can be described by an SU(3) matrix model. In several ways, the deconfining phase transition for $\mathrm{N}=3$ appears to be like that in the $N=\infty$ matrix model of Gross and Witten. 


\section{Renormalization Prescription}

We assume

$$
\log \left\langle\ell_{\mathcal{R}}\right\rangle=-f_{\mathcal{R}}^{\text {div }} N_{t}-f_{\mathcal{R}}^{c o n t}-f_{\mathcal{R}}^{\text {lat }} \frac{1}{N_{t}} .
$$

Each of the $f_{\mathcal{R}}$ 's is a power series in the renormalized coupling constant, $g^{2}$.

$$
\mathcal{Z}_{\mathcal{R}} \equiv \exp \left(-\frac{m_{\mathcal{R}}}{T}\right) \quad, \quad m_{\mathcal{R}} \equiv \frac{f_{\mathcal{R}}^{d i v}}{a}
$$

We compare two lattices at the same temperature and different lattice spacings.

$$
f_{\mathcal{R}}^{\text {div }} \rightarrow Z_{\mathcal{R}}, \quad f_{\mathcal{R}}^{\text {cont }} \rightarrow\left\langle\widetilde{\ell}_{\mathrm{N}}\right\rangle
$$

Numerically, we find

$$
f_{\mathcal{R}}^{l a t} \sim 0
$$

The success of a fit indicates that on the lattice, the divergent mass $m_{\mathcal{R}}$ does exponentiate. 


\section{Mass Term Versus $T$}

Recall from perturbation theory that the mass term scales as

$m_{\mathcal{R}} \sim C_{\mathcal{R}}$

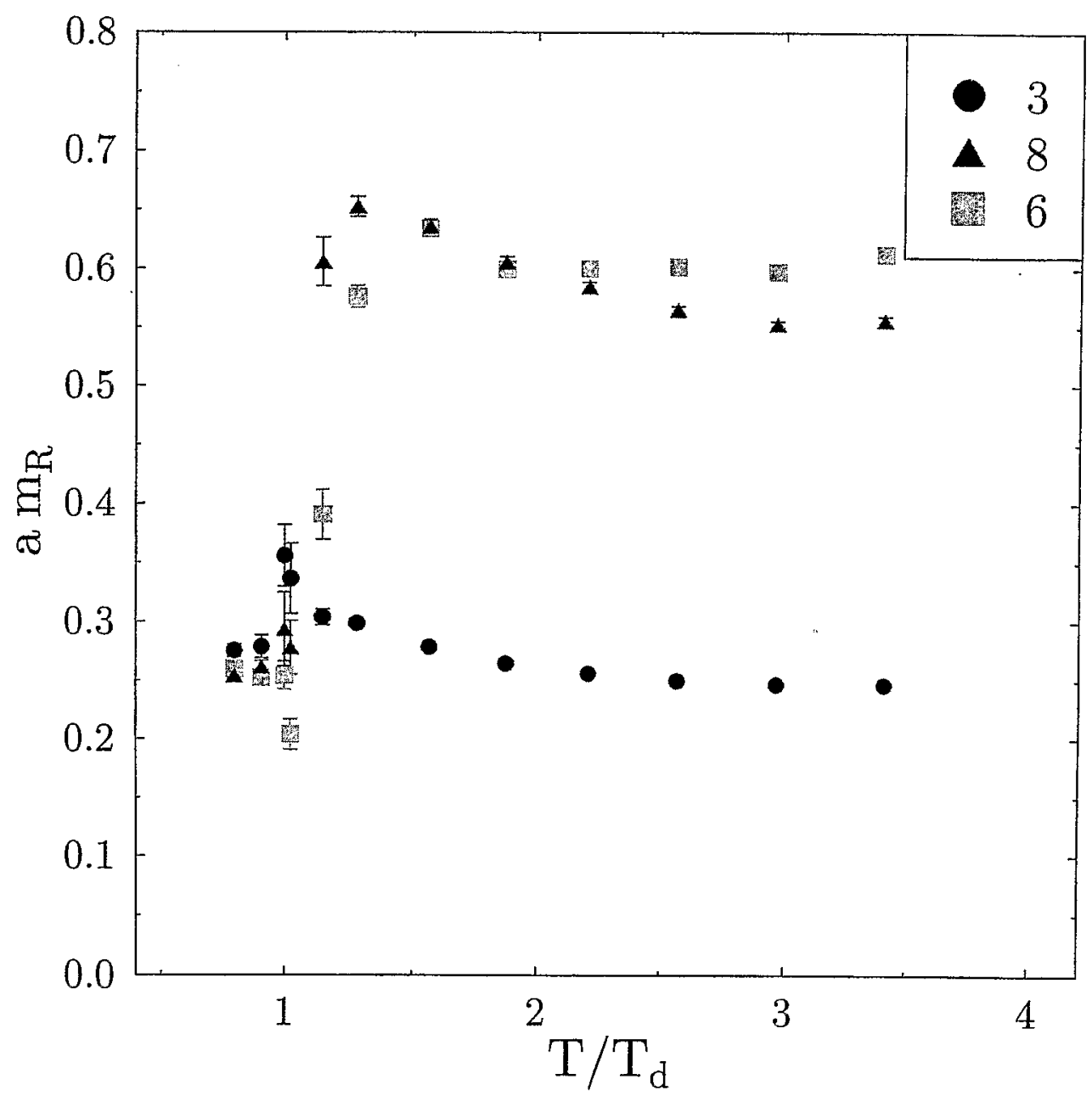




\section{Renormalized Loops}

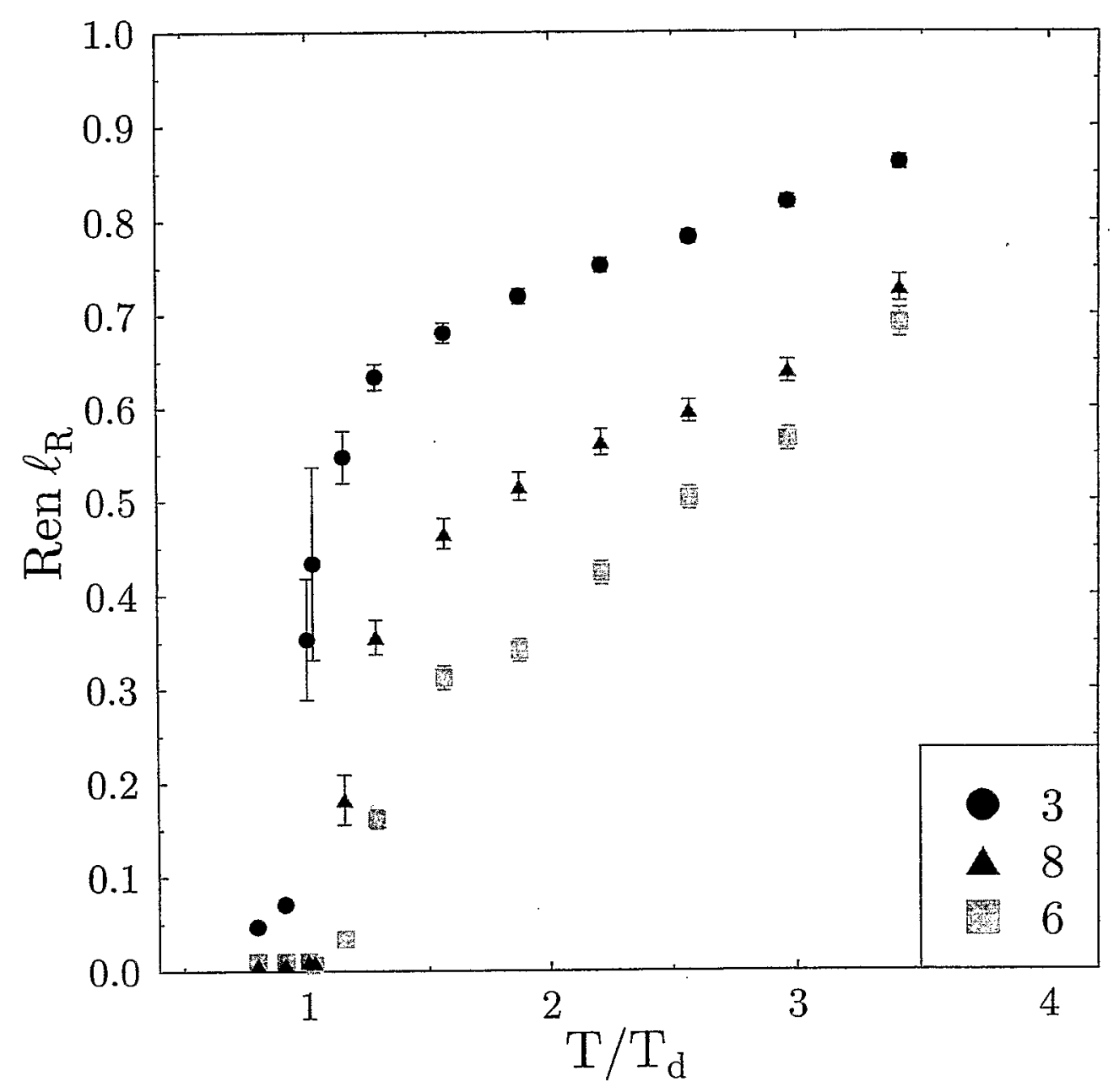

No signal for the decuplet loop. 


\section{Large $-\mathbf{N}$ and Mass of Polyakov Loop}

The connected two point function of $\ell$ is

$$
\left\langle\ell_{\mathrm{N}}^{*}(\vec{x}) \ell_{\mathrm{N}}(0)\right\rangle-\left|\left\langle\ell_{\mathrm{N}}\right\rangle\right|^{2} \sim \frac{\exp (-M|\vec{x}|)}{|\vec{x}|},|\vec{x}| \rightarrow \infty .
$$

In the confined phase, $M=\sigma / T$, where $\sigma$ is the string tension. In the deconfined phase, one can define $M=2 m_{\text {Debye }}$.

Computing $m^{2}=\partial^{2} \mathcal{V}_{m f} / \partial \ell^{2}$ :

$$
\begin{aligned}
& m_{-}^{2} \approx 2(1-\beta), \quad \beta \rightarrow 1^{-} . \\
& m_{+}^{2} \approx 4 \sqrt{\beta-1}, \quad \beta \rightarrow 1^{+} .
\end{aligned}
$$

The string tension vanishes at the transition as:

$$
\sigma(T) \sim\left(T_{d}-T\right)^{1 / 2} \quad, \quad T \rightarrow T_{d}^{-}
$$

and the Debye mass, as

$$
m_{\text {Debye }}(T) \sim\left(T-T_{d}\right)^{1 / 4}, \quad T \rightarrow T_{d}^{+} .
$$




\section{Conclusions}

- We have a method to renormalize loops in any representation.

- The method is unaffected by the presence of dynamical quarks.

- Factorization seems to be approximately true even for three colors.

- For three colors, careful measurements of the renormalized loops near $T_{d}$ will sharply constrain the couplings of the effective matrix model.

- Simulations should quickly show if the deconfining transition is near the Gross-Witten limit for four or more colors. Measure the string tension and Debye mass near $T_{d}$. 


\title{
Viscosity In Heavy Ion Collisions
}

\author{
Derek Teaney
}

February 18, 2004

- The first slide estimates the domain of applicability of hydrodynmaics. Here, I define the Sound Attenuation Length, $\Gamma_{s} \equiv \eta /(e+p) . \Gamma_{s}$ should be small compared to the smallest inverse gradient in the system. For a Bjorken expansion the smallenst inverse gradient is $1 /\left(\partial_{z} v^{z}\right)=\tau$. $\Gamma_{s}$ is of order of the transport mean free path. Thus hydrodynamics is valid when the mean free path is much smaller the system size or $\Gamma_{s} /$ tau $\ll 1$.

- The next slide garners estimates of the viscosity from the litterature. The perturbative estimate of the viscosity is approximately four times larger than an estimate based upon strongly coupled $N=4$ Supersymmetric Yang Mills [1]. The $N=4$ result is in turn is approximately four times larger than the viscosity which is needed to reproduce the observed elliptic elliptic flow with a classical Boltzmann equation [2] .

- The next slide estimates how $\Gamma_{s} / \tau$ evolves as a function of time for different temperature dependencies of the viscosity. When the viscosity is proportional to the (temperature) ${ }^{3}$ and the expansion is one dimensional Bjorken the system rapidly approaches equilibirium $-\Gamma_{s} / \tau$ decreases $1 / \tau^{2 / 3}$. In contrast when the viscosity is proportional to the temperature and the expansion is three dimensional the system quickly evolves out of equilibrium $-\Gamma_{s} / \tau$ increases like $\tau^{2}$. These simple estimates largely explain why the viscosity found in [2] is so small. Three simple models of viscosity are then introduced. In the first model visocity is proportional to (temperature) ${ }^{3}$. In the second model the viscosity is made proportional to $\frac{T}{\sigma_{0}}$. Finally, I construct a minimal model for the viscosity which is proportional to $T^{3}$ above a critical energy density and proportional to $\frac{T}{\sigma_{0}}$ below that energy density.

- The viscous hydrodynamic equations of motion are then solved numerically. The solution does not differ significantly from the ideal solution.

- Then I compare the three models of viscosity. The thin lines indicate contours of constant tranverse velocity. The minimal model and the model with constant cross section give approximately the same average transverse rapidity.

\section{References}

[1] G. Policastro, D. T. Son, A. 0. Starinets, Phys. Rev. Lett. 87, 081601 (2001) ; JHEP 0209, 043 (2002);

[2] D. Molnar and M. Gyulassy, Nucl. Phys. A 697, 495 (2002).

[3] D. Teaney, Phys. Rev. C 68, 034913 (2003). 
Ideal Hydrodynamic Simulations of Heavy Ion Collisions:

- Assume the Momentum Degradation Length $\ell_{m f p}=0$

- Write the stress energy tensor:

$$
T^{\mu \nu}=(e+p) u^{\mu} u^{\nu}+p g^{\mu \nu}
$$

- Input the equation of state: $p(e)$

- Solve the equations of motion: $\partial_{\mu} T^{\mu \nu}=0$

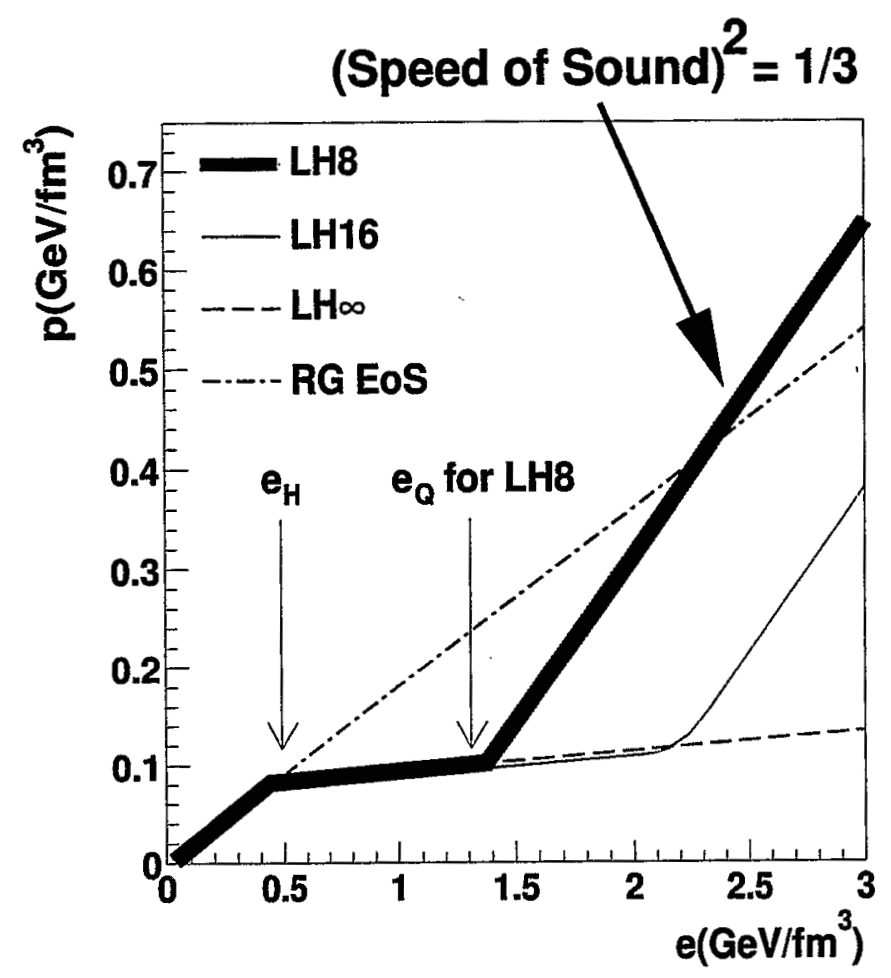


Compute $\left.v_{2}\left(p_{T}\right) \equiv \cos (2 \dot{\phi})\right\rangle_{p_{T}}$

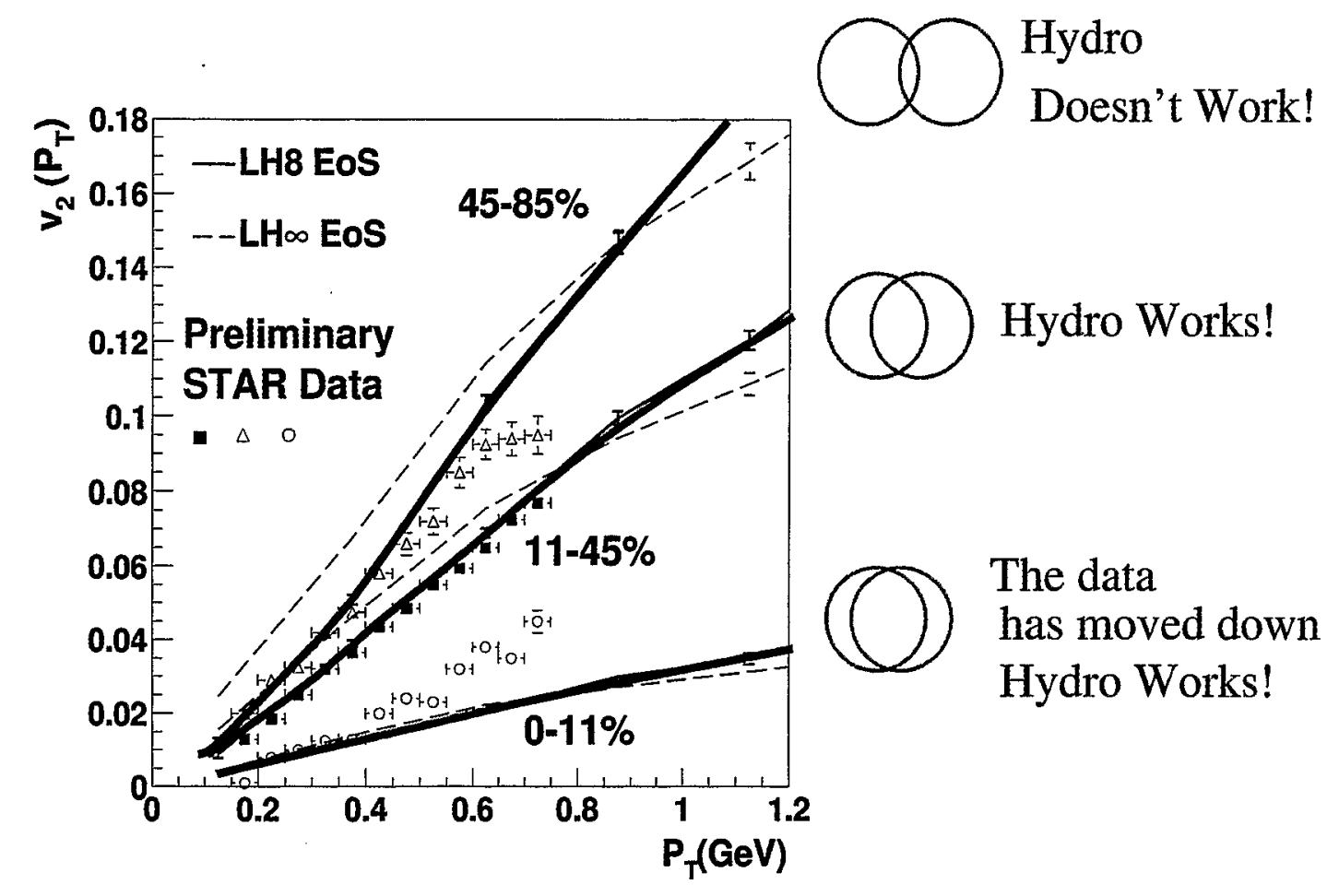

by Pasi Houvinnen

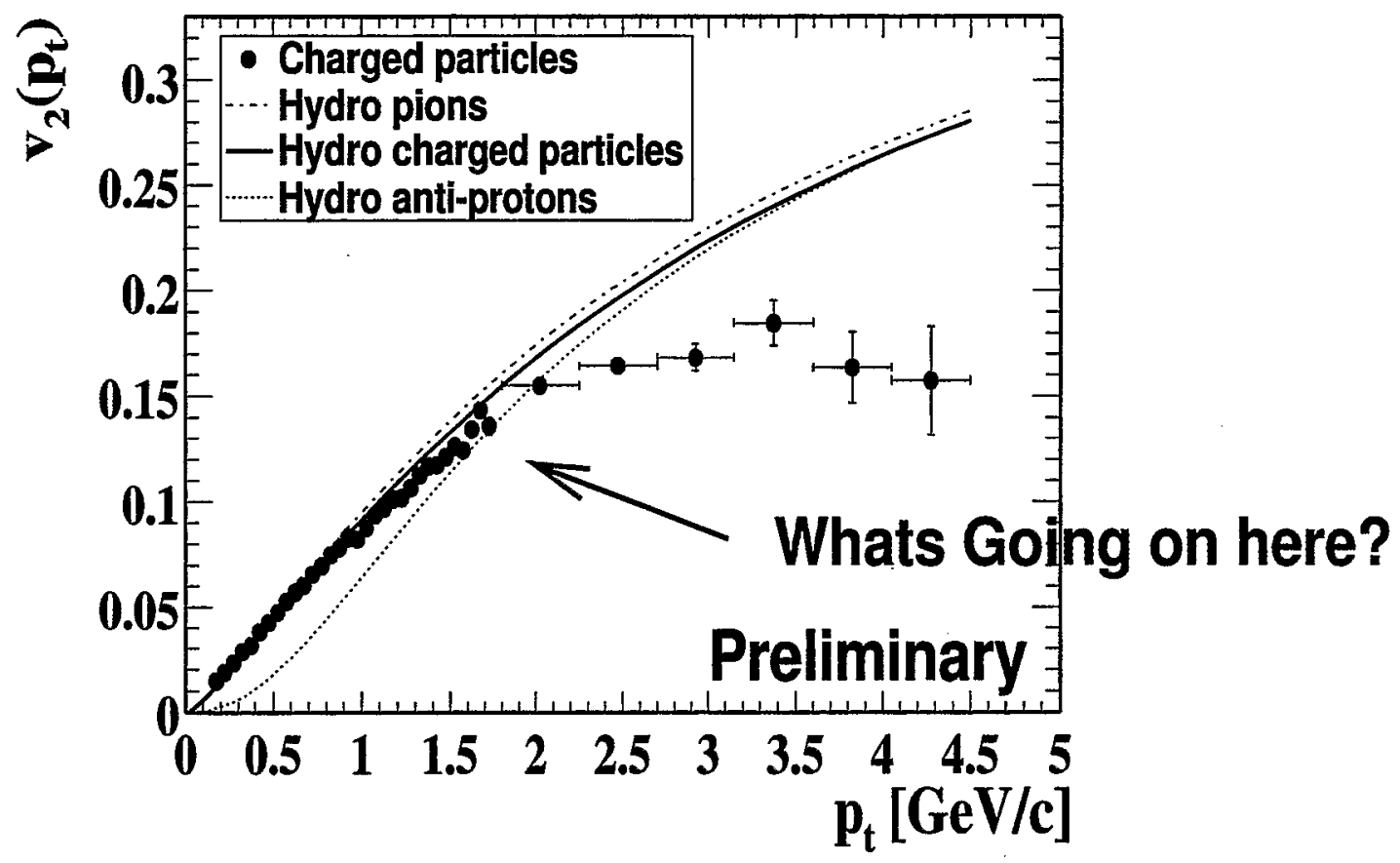


How valid is Hydro? How much Entropy is produced?

$$
\begin{array}{ll}
\frac{d(\tau s)}{d \tau}=0 & \text { (Ideal Case) } \\
\frac{d(\tau s)}{d \tau}=\frac{\frac{4}{3} \eta+\sigma}{\tau T} & \text { (Viscous Case) }
\end{array}
$$

For hydrodynamics to be valid, the entropy produced over the time scale of the system, $\tau$, must be small compared to the total :

$$
\tau \frac{\frac{4}{3} \eta+\sigma}{\tau T} \frac{1}{\tau s} \equiv \Gamma_{s} \frac{1}{\tau} \ll 1
$$

where $\Gamma_{s} \equiv \frac{\frac{4}{3} \eta+\sigma}{e+p}$ is the Sound Attenuation Length. Note $(s T=e+p)$.

Estimates of Shear Viscosity:

$$
\underbrace{T^{00}+T^{z z}}=(e+p)+\eta\left\langle\partial^{z} v^{z}\right\rangle
$$

indep. of Bag Const.

$$
\sim(e+p)[1+\underbrace{\ell_{m . f . p .}}_{1 /\left(n \sigma_{0}\right)}\left\langle\partial^{z} v^{z}\right\rangle]
$$

Using $e+p \sim n T$, then find:

$\Gamma_{s} \sim \ell_{m . f . p .} \quad$ and $\quad \eta \sim \frac{T}{\sigma_{0}}$ and $\frac{\Gamma_{s}}{\tau} \sim \frac{\ell_{m . f . p .}}{\tau} \ll 1$ 
Estimates of $\eta$ for the QGP and Heavy lon Collisions Perturbative QCD - Arnold, Moore, Yaffe.

- $\eta \approx 150 T^{3} \frac{1}{g^{4}}$.

Based upon kinetic theory of quarks and gluons. Set $\alpha_{s} \rightarrow 1 / 2$ and $m_{D} \rightarrow$ a reasonable value

$$
\left(\frac{\Gamma_{s}}{\tau}\right) \approx 0.4 \frac{1}{\tau T}
$$

Strongly Coupled conformal N=4 SYM - Son, Starinets, Policastro

- No kinetic theory exists. Like most real liquids.

$$
\left(\frac{\Gamma_{s}}{\tau}\right)=\frac{1}{3 \pi} \frac{1}{\tau T} \approx 0.11 \frac{1}{\tau T}
$$

Phenomenology - Molnar

Found could fit elliptic flow $v_{2}\left(p_{T}\right)$ only when

- $\frac{d N}{d \eta}=1000, \sigma_{0}=10 \div 20$, and $\tau_{o}=0.1 \mathrm{fm}$.

$$
\Gamma_{s}=0.421 \frac{1}{n \sigma_{0}} \quad\left(\frac{\Gamma_{s}}{\tau}\right)=0.02 \div 0.04
$$

- Constant cross section. Independent of time! 
Bjorken Solution with transverse Expansion:

Initial conditions from Kolb's Hydro: $p=\frac{1}{3} e$ and $\eta / s=0.2$
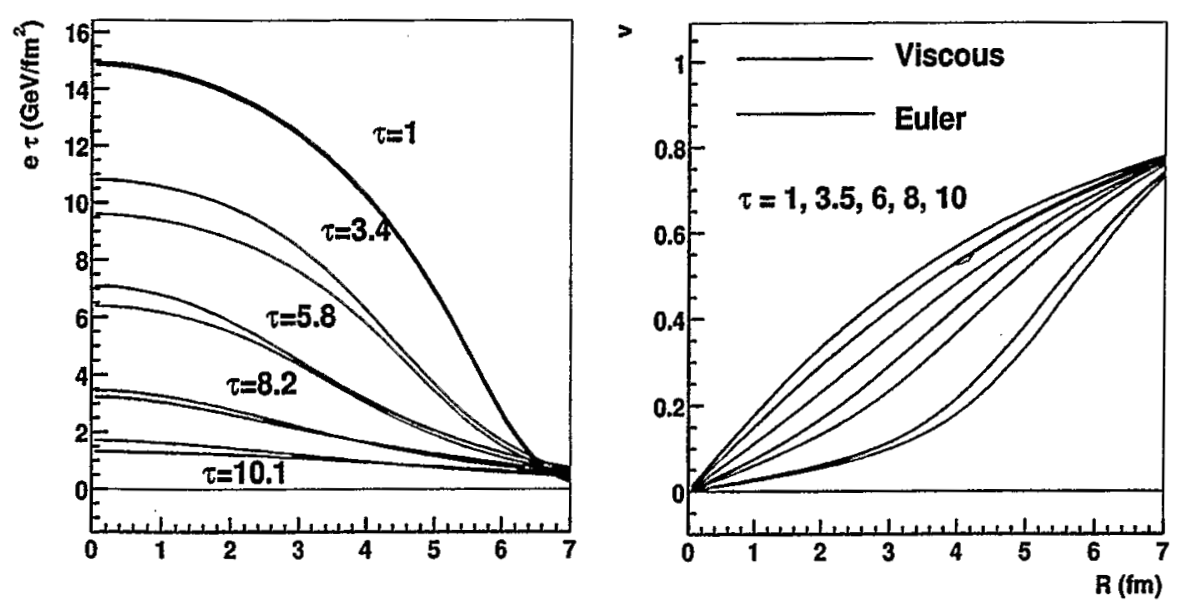

- First the viscous case does less longitudinal work.

- But, the viscous transverse velocity grows more rapidly because the transverse pressure is larger

- The larger transverse velocity then reduces the viscous energy density more quickly than Euler hydro.

Viscous corrections do NOT integrate to give an $O(1)$ change to the flow. 
Summary:

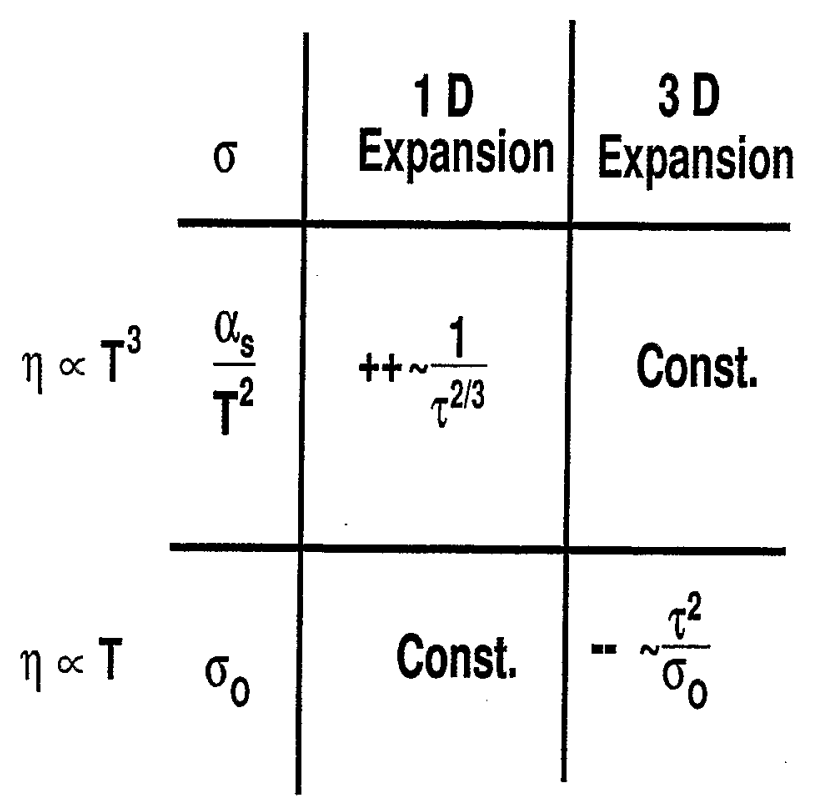

Three models of viscosity:

- A scale free model: $\eta \propto T^{3}$

$$
\eta=\frac{1}{5} s
$$

- Constant cross section:

$$
\eta=1.2 \frac{T}{\sigma_{0}} \text { with } \sigma_{0}=10 \mathrm{mb} .
$$

- A minimal model: $e_{c}=1 \mathrm{GeV} / \mathrm{fm}^{3}$

$$
\eta= \begin{cases}1.2 \frac{\mathrm{T}}{\sigma_{0}} & \text { for } e<e_{c} \\ \frac{1}{5} \mathrm{~s} & \text { for } e>e_{c}\end{cases}
$$


Compare the three models of viscosity:
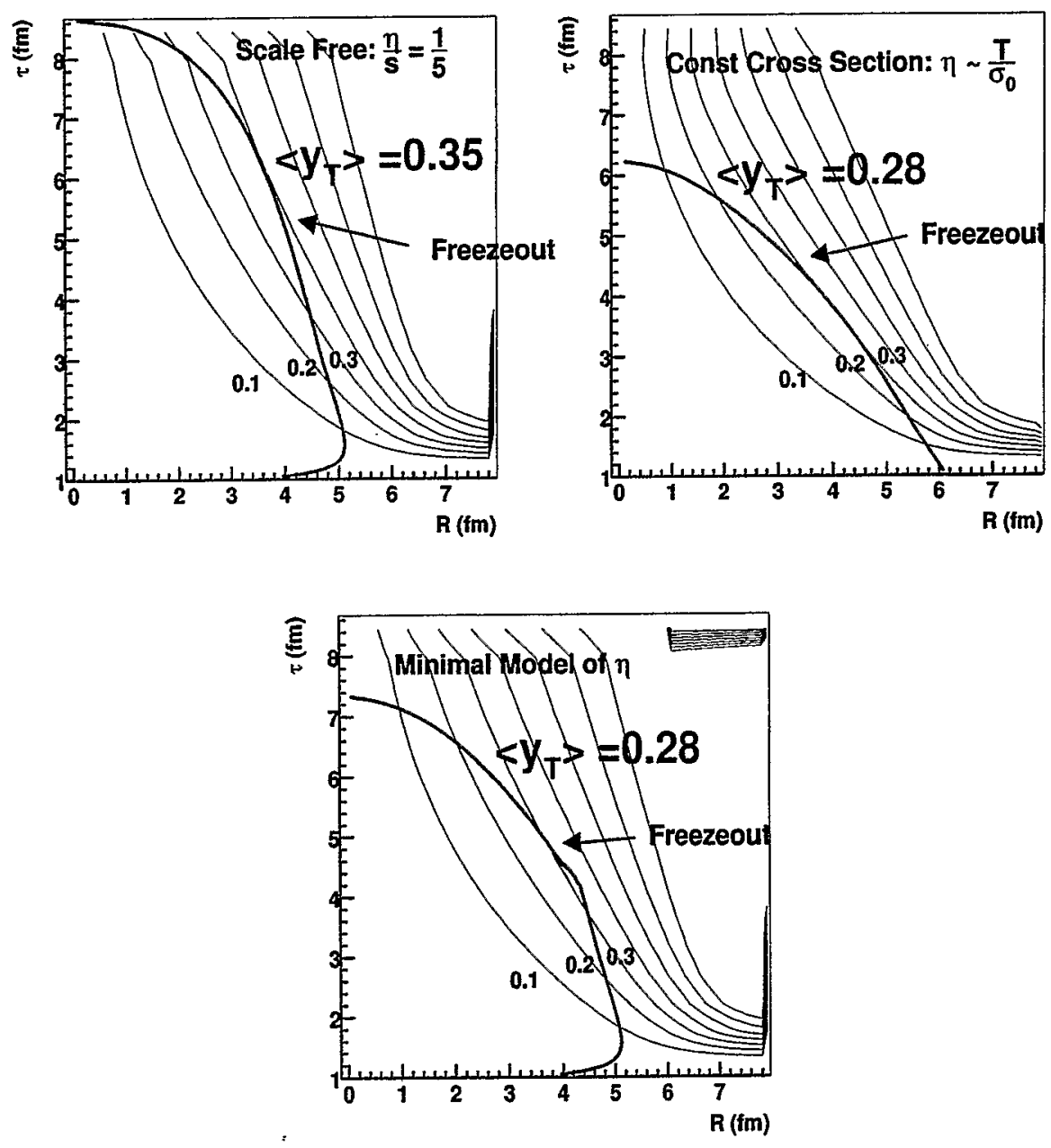

The minimal model of $\eta$ and the Const X.-section model of $\eta$ yield the same amount of radial flow. 
Brookhaven, 12 Feb 2004

\title{
Pressure of hot QCD - is $g^{6}$ and beyond calculable?
}

K. Kajantie, Helsinki

\author{
In collaboration with: \\ Www physics.helsinki: fi/rkajantie/dlectures/ton. \\ A. Hietanen, M. Laine, K. Rummukainen, Y. Schröder

Here $p(T, \mu=0), \quad \mu>0$ has been done by Aleksi Vuorinen 
The perturbative expression for the pressure is now known up to $g^{6} \log g$ :

$$
\begin{aligned}
& p / p_{\mathrm{SB}}=1 \quad \text { Stefan-Boltzmann } \\
& \begin{array}{lll}
+g^{2} & \text { 2-loop (Shuryak 78) }
\end{array} \\
& +g^{3} \quad \text { resum 2-loop (Kapusta 79) } \\
& +g^{4} \ln 1 / g \text { resum 2-loop (Toimela 83) } \\
& +g^{4} \quad \text { resum 3-loop (Arnold, Zhai 94) } \\
& +g^{5} \quad \text { resum 3-loop (Kastening, Zhai 95) } \\
& \begin{array}{lll}
+g^{6} \ln 1 / g & \text { resum 4-loop } & \text { (KLRSO2) } \\
+g^{6} & \text { not computable in PT!! } & \text { (Linde 80; this talk) }
\end{array} \\
& +g^{7} \ldots \quad \ldots \quad \text { (this talk) } \\
& p_{\mathrm{SB}}=\frac{\pi^{2}}{90}\left(16+\frac{21}{2} N_{f}\right) T^{4}
\end{aligned}
$$

Even though the $g^{6}$ coefficient is not calculable in PT, it is calculable - as is the non-perturbative sum starting $g^{7}$.
Effective theory approach: Braaten-Nito

$$
\begin{aligned}
& p_{\text {c⿻o }}(T) / T=\left(p_{E}+p_{M}+p_{G}\right) / T= \\
& =T^{3}\left[1+g^{2}(\Lambda)+g^{4}(\Lambda)+\left(\log \frac{\Lambda}{T}+a \log \frac{T}{\Lambda_{E}}\right) g^{6}(\Lambda)\right]+ \\
& +m_{E}^{3}+m_{E}^{2} g_{E}^{2}+m_{E} g_{E}^{4}+\left(a \log \frac{\Lambda_{E}}{m_{E}}+b \log \frac{m_{E}}{\Lambda_{M}}\right) g_{E}^{6}+ \\
& +g_{M}^{6} \log \frac{\Lambda_{M}}{g_{M}^{2}}
\end{aligned}
$$

Sequence of three theories:

- Full hot 4d QCD

- 3d gauge+adjoint Higgs theory, $S\left[A_{i}, A_{0}\right]$

- 3d gauge theory, $S\left[A_{i}\right]=\frac{1}{4} F_{i j}^{2}$

carefully matched in the UV. (Matching will be also the main point in the next talk by Vepsäläinen)

\section{How?}


Perturbative result (known for all $N_{f}$ ):

$$
a \equiv \frac{\alpha_{s}(\bar{\mu})}{\pi} \quad N_{f}=0
$$$$
p_{\mathrm{QCD}}(T) / p_{\mathrm{SB}}(T)=1-\frac{15}{4} a+30 a^{3 / 2}+
$$$$
+\left(237.2+\frac{135}{2} \log a-\frac{11}{2} \frac{15}{4} \log \frac{\bar{\mu}}{2 \pi T}\right) a^{2}
$$$$
+\left(-799.1+\frac{495}{2} \log \frac{\bar{\mu}}{2 \pi T}\right) a^{5 / 2}
$$$$
+\left(-659.2+742.5 \log \frac{\bar{\mu}}{2 \pi T}-475.6\right) \dot{a}^{3} \log a
$$$$
+\left(-\frac{1815}{16} \log ^{2} \frac{\bar{\mu}}{2 \pi T}+2932.6 \log \frac{\bar{\mu}}{2 \pi T}+p_{6}\right) a^{3}+\ldots
$$

$$
\begin{aligned}
& -475.6=-\frac{17415}{16}+\frac{63585}{1024} \pi^{2} \\
& -659.2=-\frac{49005}{32}+\frac{198855}{2048} \pi^{2}-\frac{1485}{2}\left(\log 2-\gamma_{E}\right)
\end{aligned}
$$

Better: do NOT expand $m_{E}^{3} / T^{3}=\left(g^{2}+g^{4}+. .\right)^{3 / 2} \sim g^{3}+g^{5}+. .1$

\section{Now that one has computed that}

$$
p(T) / p_{\mathrm{sB}}=\ldots+0.03738 g^{6} \log \frac{1}{g}+\ldots
$$

one can at least fit the $g^{6}$ coefficient:

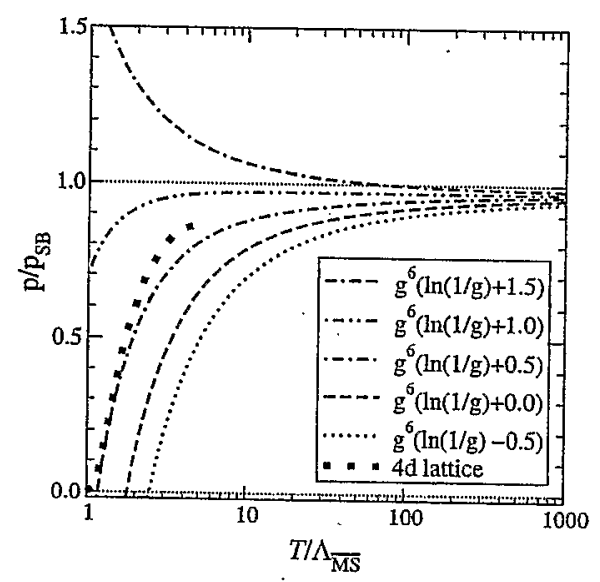

$\log (1 / g)+0.7$ gives a good fit, but is the 0.7 only $g^{6}$ ? 


\section{Pure 3d SU(3)}

$$
\begin{aligned}
& Z=\exp \left[\frac{p_{G}(T)}{T} V_{3}\right] \\
= & \int \mathcal{D} A_{i} \exp \left(-\int \mathrm{d}^{d} x \frac{1}{4} F_{i j}^{a} F_{i j}^{a}\right) \quad \overline{\mathrm{MS}} \\
= & \int \mathcal{D} U_{i} \exp (-\beta \Sigma[1-\square]) \quad \text { lattice }
\end{aligned}
$$

MS Scheme: KLRS, hep-ph/0211921, Schröder, hep-ph/0211268

$$
\frac{p_{\mathrm{G}}(T)}{T \mu^{-2 \varepsilon}}=\frac{d_{A} C_{A}^{3}}{(4 \pi)^{4}} g_{\mathrm{M}}^{6}\left[\left(\frac{43}{96}-\frac{157 \pi^{2}}{6144}\right)\left(\frac{1}{\epsilon}+8 \ln \frac{\bar{\mu}}{2 C_{A} g_{M}^{2}}\right)+\beta_{G}+. .\right]
$$

For $\overline{\mathrm{MS}}$ with no scale $1 / \epsilon_{\mathrm{UV}}-1 / \epsilon_{\mathrm{R}}=0$. To separate the UV divergence, shield IR by introducing $m_{\text {gluon }}$, use arbitrary $\xi$. Then $\beta_{G}=\beta_{G}\left(m_{\text {gluon }}, \xi\right)$.

Lattice: Take derivative of $p_{G}(T) / T$ w.r.t $\beta$, determine $\langle\square\rangle$, integrate back.

Remarkable recent progress using stochastic perturbation theory Di Renzo, Mantovi, Miccio, Schröder, hep-lat030s111.

Determination of $\beta_{G}$ and $p_{6}$ seems feasible! Avoid doing analytically 4-loop lattice perturbation theory!
After integration:

$$
\begin{aligned}
\frac{p_{G}(T)}{T}= & 3 c \frac{\log g_{3}^{2} a}{a^{3}}+\quad c=d_{A} / 3 \\
& +\frac{c_{1}}{2} \frac{g_{3}^{2}}{a^{2}}+\quad c_{1}^{\text {Hellor-Karsch }}=1.94862 \\
& +\frac{c_{2}}{24} \frac{g_{3}^{4}}{a}+\quad c_{2}=6.7 \pm 0.2 \\
& +\frac{g_{3}^{6}}{216}\left(c_{3} \log \frac{6}{g_{3}^{2} a}+\frac{1}{3} c_{3}+\tilde{c}_{3}\right) \\
c_{3}= & 0.9 \pm 0.4 \quad \tilde{c}_{3}=23 \pm 5
\end{aligned}
$$

The value of $c_{3}$ agrees with the analytic one!! Next

- Do $\overline{\mathrm{MS}}$ in finite $V$ or

- Do stochastic perturbation theory with $m_{\text {qluon }}, \xi$

to get the Linde coefficient $\beta_{\mathrm{G}}$. 
3d SU(3) gauge + adjoint Higgs theory:

$$
\begin{gathered}
\exp \left[\frac{p_{M}(T)}{T} V_{3}\right]=\int \mathcal{D} A_{i}^{a} \mathcal{D} A_{0}^{a} \exp \left\{-\int d^{3} x \times\right. \\
{\left[\frac{1}{4} F_{i j}^{a} F_{i j}^{a}+\frac{1}{2}\left(D_{i} A_{0}\right)^{a}\left(D_{i} A_{0}\right)^{a}+\right.} \\
+\underbrace{\frac{1}{16 \pi^{2}}\left(22 \log \frac{5.371 T}{\Lambda_{\overline{M S}}}+9\right)}_{\equiv m_{E}^{2} / g_{E}^{4}=y \sim 1 / g^{2}} \frac{1}{2} A_{0}^{a} A_{0}^{a}+ \\
+\underbrace{\frac{3}{44 \log \left(5.371 T / \Lambda_{M S}\right)}}_{\equiv \lambda_{E} / g_{E}^{2} \equiv x} \frac{1}{4}\left(A_{0}^{a} A_{0}^{a}\right)^{2}+\frac{\cdots}{T}]\}
\end{gathered}
$$

$A_{i}, A_{0}, \mathrm{x}$, dimensionless, $D_{i}=\partial_{i}+i A_{i}$

Here $m_{E}^{2}, g_{E}^{2}, \lambda_{E}$ are matched to $4 \mathrm{~d}$ theory using next-toleading-order optimised perturbation theory.

Both $m_{E}^{2}$ and $\lambda_{F}^{2}$ are given by $T / \Lambda_{\mathrm{MS}}$.
MS: KLRS, hep-ph/0304048

$$
\begin{aligned}
& \frac{p_{M}(T)}{T \mu^{-2 \epsilon}}=m_{E}^{3}+g_{E}^{2} m_{E}^{2}+g_{E}^{4} m_{E}+ \\
&+\frac{d_{A} C_{A}^{3}}{(4 \pi)^{4}} g_{E}^{6}\left[\left(\frac{43}{32}-\frac{491 \pi^{2}}{6144}\right)\left(\frac{1}{\epsilon}+8 \ln \frac{\bar{\mu}}{2 m_{E}}\right)+\beta_{M}+\mathcal{O}(\epsilon)\right] \\
& \beta_{M}=-\frac{311}{256}-\frac{43}{32} \ln 2-\frac{19}{6} \ln ^{2} 2+\frac{77}{9216} \pi^{2}- \\
& \quad-\frac{491}{1536} \pi^{2} \ln 2+\frac{1793}{512} \zeta(3)+\gamma_{10} \\
&=-1.562519+\gamma_{10}=-1.391512 \ldots
\end{aligned}
$$

Lattice: Take derivative of $p_{M}(T) / T$ w.r.t $m_{E}^{2}$, determine $\left\langle A_{0}^{a} A_{0}^{a}\right\rangle$, integrate back.

Now we know 1-,2-,3-,4-loop perturbative results analytically (for $\langle\square\rangle$ only $1-$ and 2 -loop)! If

$$
\frac{p_{M}}{T} \sim m_{E}^{3}+g_{E}^{2} m_{E}^{2}+g_{E}^{4} m_{E}+g_{E}^{6}\left(\log \frac{\bar{\mu}}{m_{E}}+\beta_{M}\right)+\frac{g_{E}^{8}}{m_{E}}+\ldots
$$

then (dimless terms; $\beta_{M}$ disappears!))

$$
\left\langle\frac{A_{0}^{2}}{g_{E}^{2}}\right\rangle=-\frac{m_{E}}{\pi g_{E}^{2}}+1+\frac{g_{E}^{2}}{m_{E}}+\frac{g_{E}^{4}}{m_{E}^{2}}+\frac{g_{E}^{6}}{m_{E}^{3}}+\ldots
$$

It is the last term $+\ldots$ we want to measure! 
Continuum extrapolation: $\beta_{G}=6 / g_{E}^{2} a \rightarrow \infty$.

Choose $y=m_{E}^{2} / g_{E}^{4} \sim 1 / g^{2}=1.14-6.39$, corresponding to $T \sim 100-10^{20} \Lambda_{\mathrm{MS}}$. Leading term was $-\sqrt{y} / \pi$.

$\overrightarrow{\mathrm{t}}$

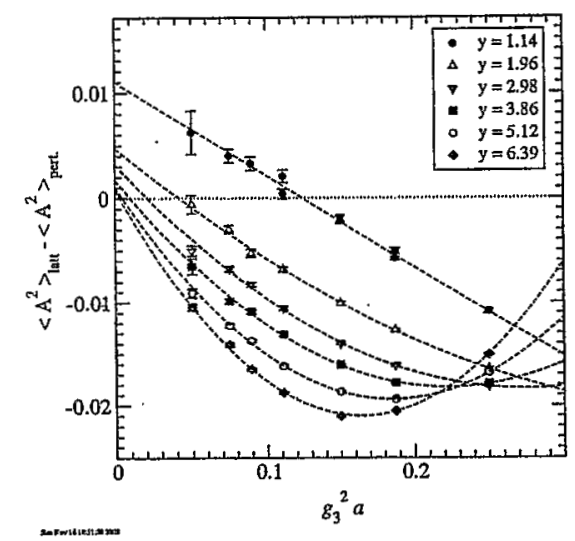

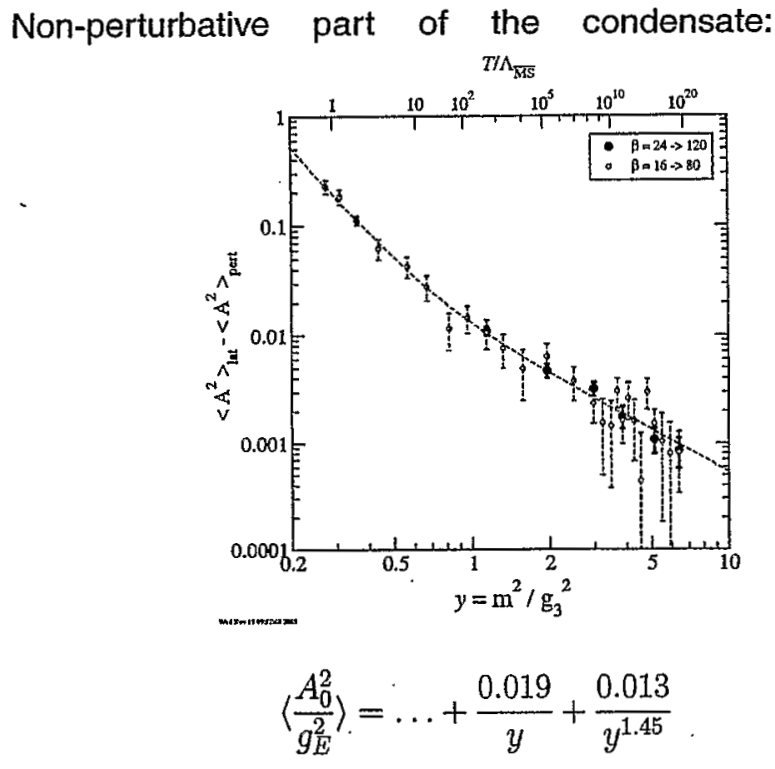

$(0.019 / y$ is the 4 loop result, subtracted in fig). 
Integrating the nonperturbative additional term in $\left\langle A_{0}^{2}\right\rangle$ over $y=m_{E}^{2} / g_{E}^{4}=1 / g^{2}$ one obtains the additional free energy

$$
-\frac{0.013}{0.45 y^{0.45}}
$$

which adds to $p / p_{\mathrm{SB}}$ the last term in

$$
\begin{aligned}
\frac{p(T)}{p_{\mathrm{SB}}} & =\ldots+0.03738 g^{6} \log \frac{1}{g}+0.01645 g^{6.9} \\
& =\ldots+0.03738 g^{6}\left(\log \frac{1}{g}+0.44 g^{0.9}\right)
\end{aligned}
$$

$\begin{array}{ll}\text { un } \quad \begin{array}{l}1.7>g>1.3 \\ \text { when } \\ 2<T / \Lambda<10 \\ \text { Thus } \\ 0.44 g^{0.9}\end{array} \\ & \text { s. }\end{array}$$$
0.44 g^{0.9}
$$

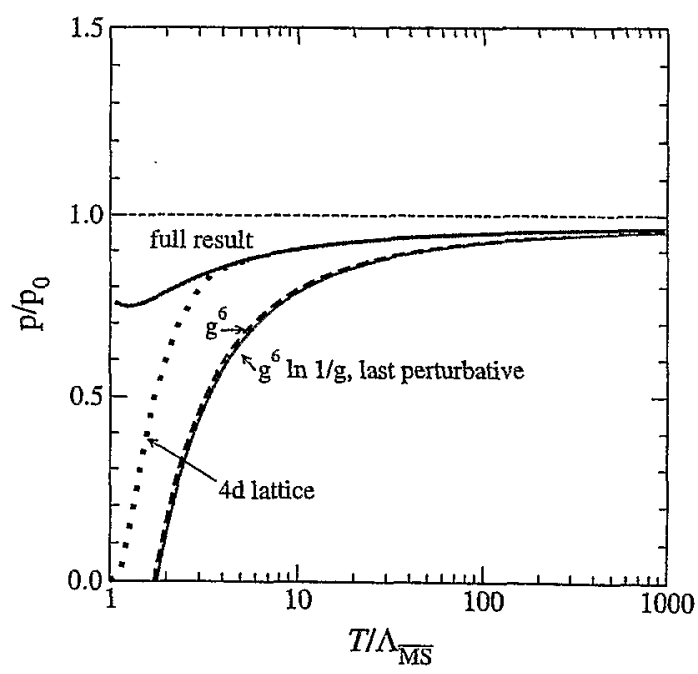

That

$$
+0.03738 g^{6}\left(\log \frac{1}{g}+0.44 g^{0.9}\right)
$$

gives a good fit to data indicates that

- the order $g^{6}$ term is small

- perturbation theory + non-perturbative $3 d$ effects describe physics down to $\approx 3 T_{c}$. And this is a first-principle computation in field theory.

Why is the $g^{6}$ coefficient small? Big Linde term $\beta_{G}$ which is effectively cancelled by -big terms from matching?

One should work out the $g^{6}$ coefficient!

Small Corrections from $\left\langle A_{0}^{4}\right\rangle$ (start at $g^{6}$ ), higher dimensional terms truncated in the effective theory $S\left[A_{i}, A_{0}\right]$, say, $A_{0}^{2} F_{i j}^{2}$, (start at $\left.g^{7}\right) \ldots$ 
What remains to be done for $g^{6}$ ?

$$
\begin{gathered}
+\frac{g^{6}}{(4 \pi)^{4}}\left\{\beta_{\mathrm{E} 1}-\frac{1}{4} d_{A} \alpha_{\mathrm{E} 4}\left[\left(d_{A}+2\right) \beta_{\mathrm{E} 4}+\frac{2 d_{A}-1}{N_{c}} \beta_{\mathrm{E} 5}\right]\right. \\
-d_{A} C_{A}\left[\frac{1}{4}\left(\alpha_{\mathrm{EE}}+\alpha_{\mathrm{E5}} \alpha_{\mathrm{E}}+3 \alpha_{\mathrm{E} 4} \alpha_{\mathrm{E} 7}+\beta_{\mathrm{E} 2}+\alpha_{\mathrm{E} 4} \beta_{\mathrm{E} 3}\right)\right. \\
\left.+\left(\alpha_{\mathrm{EG}}+\alpha_{\mathrm{E} 4} \alpha_{\mathrm{E} 7}\right)\left(\frac{1}{4 \epsilon}+\ln \frac{\bar{\mu}}{2 g T \alpha_{\mathrm{E}}^{1 / 2}}\right)\right] \\
\left.+d_{A} C_{A}^{3}\left[\beta_{\mathrm{M}}+\beta_{\mathrm{G}}+\alpha_{\mathrm{M}}\left(\frac{1}{\epsilon}+8 \ln \frac{\bar{\mu}}{2 g T \alpha_{\mathrm{E} 4}^{1 / 2}}\right)+\alpha_{\mathrm{G}}\left(\frac{1}{\epsilon}+8 \ln \frac{\bar{\mu}}{2 g^{2} T C_{A}}\right)\right]\right\}
\end{gathered}
$$

$\beta_{\mathrm{E} 1}:$ Calculate in full $4 \mathrm{~d}$ theory in the $\overline{\mathrm{MS}}$ scheme the order $g^{6}$ term for the pressure. Need 4-loop sum-integrals, should be doable. IR $1 / \epsilon$ poles appear which precisely cancel those above.

$\beta_{\mathrm{E} 2}, \beta_{\mathrm{Eg}}:$ Calculate in full $4 \mathrm{~d}$ theory the order $\epsilon 2$-loop terms in $m_{E}^{2}, g_{E}^{2}$.

$\beta_{\mathrm{G}}$ : Use stochastic pefturbation theory in pure $\mathrm{SU}(3)$ to relate $\overline{\mathrm{MS}}$ and lattice and to find this Linde coefficient.

\section{Conclusions}

- The perturbative computation of the pressure $p(T$ hot QCD has been driven as far it can be

- There exists a definite and realistic scheme for co ing the order $g^{6}$ term. One needs both numerical and analytic computations.

- Inclusion of $3 d$ nonperturbative effects beyond tends the agreement with present lattice data dc $3 T_{c}$.

- Can one ever have such accurate and controllab proximations for time-dependent kinetic problems: 


\title{
Mesonic screening masses at high temperature
}

\author{
Mikko Vepsäläinen \\ University of Helsinki
}

February 122004

\begin{abstract}
A dimensionally reduced effective theory analogous to NRQCD is used to calculate screening lengths for various quark-antiquark operators. At next-to-leading order we find a small gauge invariant and infrared safe correction to the free theory value $2 \pi T$, differing in sign from the $4 \mathrm{~d}$ lattice computations.
\end{abstract}




\section{Fermionic Lagrangian}

Write $\psi=\left(\begin{array}{c}\chi \\ \phi\end{array}\right)$ and solve the EOM for heavy components near $p_{3}= \pm i p_{0}$ :

$$
\Rightarrow \mathcal{L}_{\mathrm{f}}=i \chi^{\dagger}\left(M-g_{\mathrm{E}} A_{0}+D_{t}-\frac{\nabla_{\perp}^{2}}{2 p_{0}}\right) \chi+i \phi^{\dagger}\left(M-g_{\mathrm{E}} A_{0}-D_{t}-\frac{\nabla_{\perp}^{2}}{2 p_{0}}\right) \phi
$$

The particle $(\chi)$ and antiparticle $(\phi)$ sectors are clearly separated and propagate only forward/backward in the $x_{3}$ direction:

$$
\left\langle\chi_{u}(x)^{*} \chi_{v}(y)\right\rangle=-i \delta_{u v} \theta\left(x_{3}-y_{3}\right) \frac{p_{0}}{2 \pi\left|x_{3}-y_{3}\right|} e^{-M\left|x_{3}-y_{3}\right|-\frac{p_{0}\left(x_{\perp}-y_{\perp}\right)^{2}}{2\left|x_{3}-y_{3}\right|}}
$$


In the reduced theory the Matsubara frequency $\pi T \equiv M$ acts as a mass term. NLO $M$ becomes a parameter that needs matching. We did this by comparing the locations of poles in fermion propagators, which is both a gauge invariant and an IR safe method.

To preserve the correct power counting $\pi T \gg \boldsymbol{p}_{\perp}$ while integrating over $\boldsymbol{p}_{\perp}$, we expand the ET quark propagators in $\boldsymbol{p}_{\perp} / p_{0}$.

$$
\stackrel{\text { हक }}{\longrightarrow}=g_{\mathrm{E}}^{2} C_{\mathrm{F}} \int \frac{\mathrm{d}^{3-2 \epsilon} q}{(2 \pi)^{3-2 \epsilon}} \frac{1}{M+i p_{3}-i q_{3}} \frac{1}{q^{2}+\lambda^{2}}
$$

Matching the QCD and NRQCD results gives the mass parameter in effective theory:

$$
M=\pi T+g^{2} T \frac{C_{\mathrm{F}}}{8 \pi}
$$




$$
\begin{aligned}
V(\boldsymbol{r}) & =\text { Ded } \\
& =g_{\mathrm{E}}^{2} C_{\mathrm{F}} \int \frac{\mathrm{d}^{2-2 \epsilon}}{(2 \pi)^{2-2 \epsilon}}\left[\frac{1}{q^{2}+\lambda^{2}}\left(1-e^{i q \cdot r}\right)-\frac{1}{q^{2}+m_{\mathrm{E}}^{2}}\left(1+e^{i q \cdot r}\right)\right]
\end{aligned}
$$

$\overline{8}$

With this specific combination of signs, the potential is both IR and UV finite. Taking the limit $\epsilon \rightarrow 0, \lambda \rightarrow 0$ gives

$$
V(\boldsymbol{r})=g^{2} T \frac{C_{\mathrm{F}}}{2 \pi}\left[\ln \frac{m_{\mathrm{E}} r}{2}+\gamma_{\mathrm{E}}-K_{0}\left(m_{\mathrm{E}} r\right)\right] .
$$




\section{Results}

Solving the corresponding Schrödinger equation numerically gives

$$
\frac{E-2 M}{g_{\mathrm{E}}^{2} \frac{C_{\mathrm{F}}}{2 \pi}}= \begin{cases}0.16368014 & \left(N_{f}=0\right) \\ 0.38237416 & \left(N_{f}=2\right) \\ 0.46939139 & \left(N_{f}=3\right)\end{cases}
$$

$$
\Rightarrow \quad E \approx 2 \pi T+0.14083730 g^{2} T \quad\left(N_{f}=0, \text { quenched }\right)
$$

At $T \sim 2 T_{c}$ and $N_{f}=0 g_{\mathrm{E}}^{2} / T \approx 2.7,{ }^{1}$ only a $5 \%$ correction.

\footnotetext{
${ }^{1}$ Kajantie, Laine, Rummukainen and Shaposhnikov, Nucl.Phys. B503 (1997) 357 


\section{Conclusions, outlook}

- Consistent method for calculating spatial correlators of fermionic operators

- Systematics clear

穴

- Sign differs from that of $4 d$ lattice $Q C D$

- $\mathrm{NRQCD}_{3}$ should be easy to implement on lattice

- Flavour singlets`still problematic 


\title{
Gluon screening masses
}

\author{
A.Nakamura and T.Saito \\ RIISE, Hiroshima Univ.
}

LATTICE QCD AT FINITE TEMPERATURE AND DENSITY

RBRC, Feb. 8 - 12, 2004

Abstract:

Lattice QCD calculations Tc 5Tc confront Experimental data from RHIC. We report our simulation results on gluon propagators and color dependent $¥ b a r\{q\} q$ and di-quark potentials using the stochastic gauge fixing algorithm by Zwanziger in the quench approximation.

We extract the gluon screening masses from the gluon propagators. They have little gauge parameter dependence. These non-perturbatively determined values are important not only for the theoretical base, but also for the QGP phenomenology. The screening masses behave approximately $\mathrm{me} / T \sim \mathrm{Cg}(T), \mathrm{mm} / \mathrm{T} \sim \mathrm{Cg}(T)^{\wedge} 2$.

The color singlet has wider range in $\mathrm{R}$ comparing with the standard color averaged one. We study the Temperature dependence of the force. The antisymmetric di-quark potential is attractive, and its force is about half of that of the singlet $¥ \mathrm{bar}\{q\} q$ force. 


\section{Fitting to extrapolate mass}

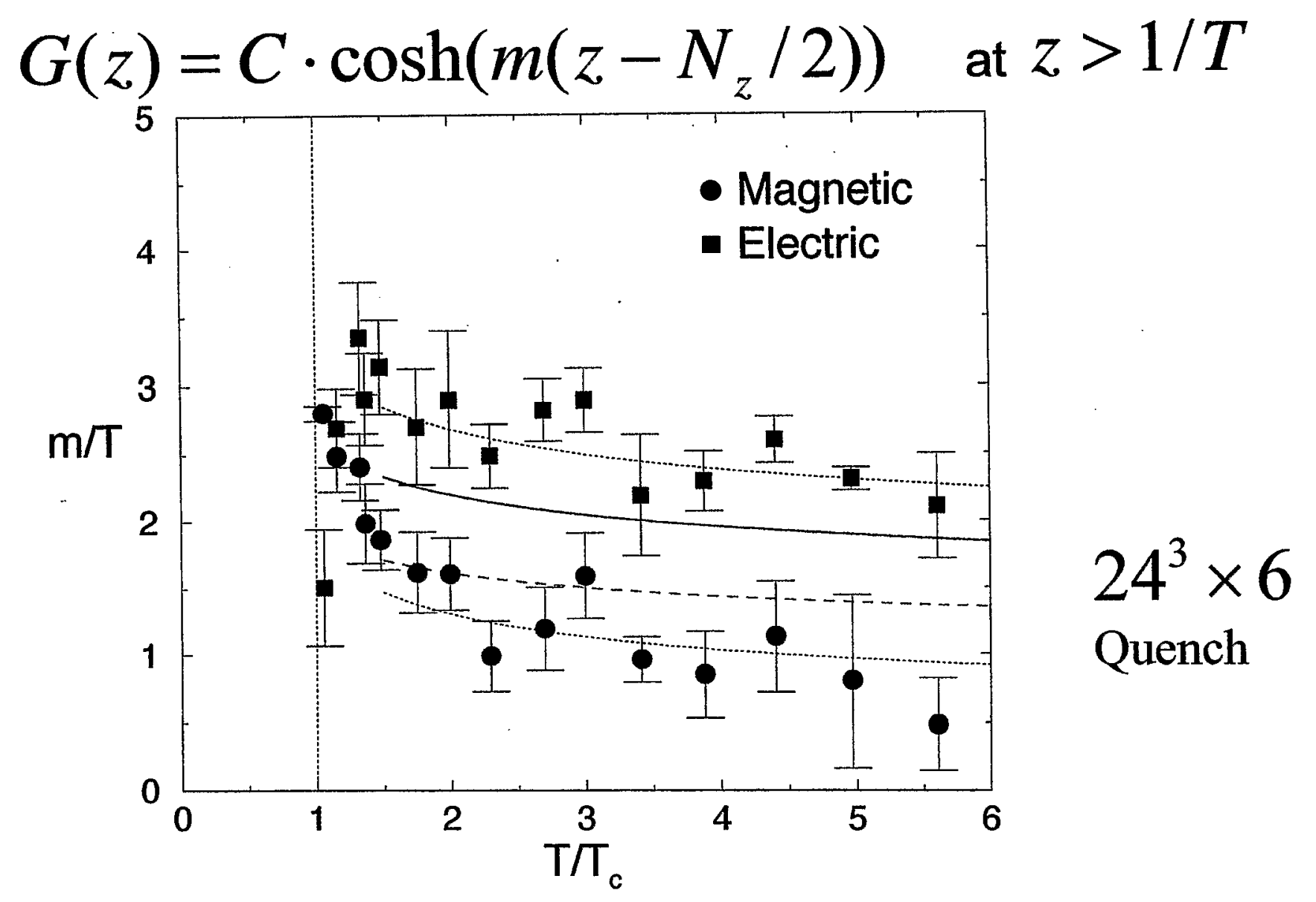




\section{Color-dependent Forces}

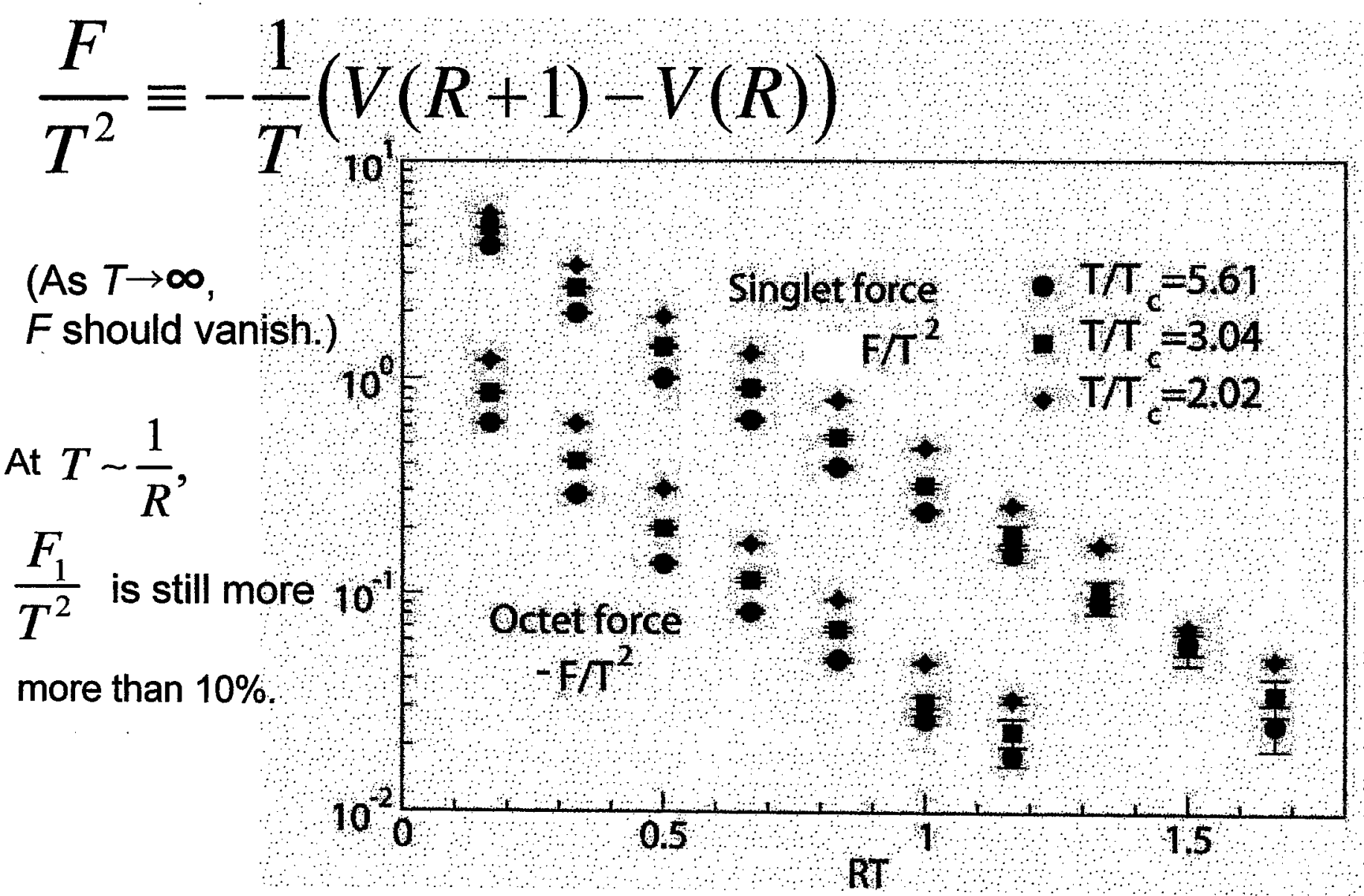




\section{$\bar{q} q$ and $q q$ Potentials}

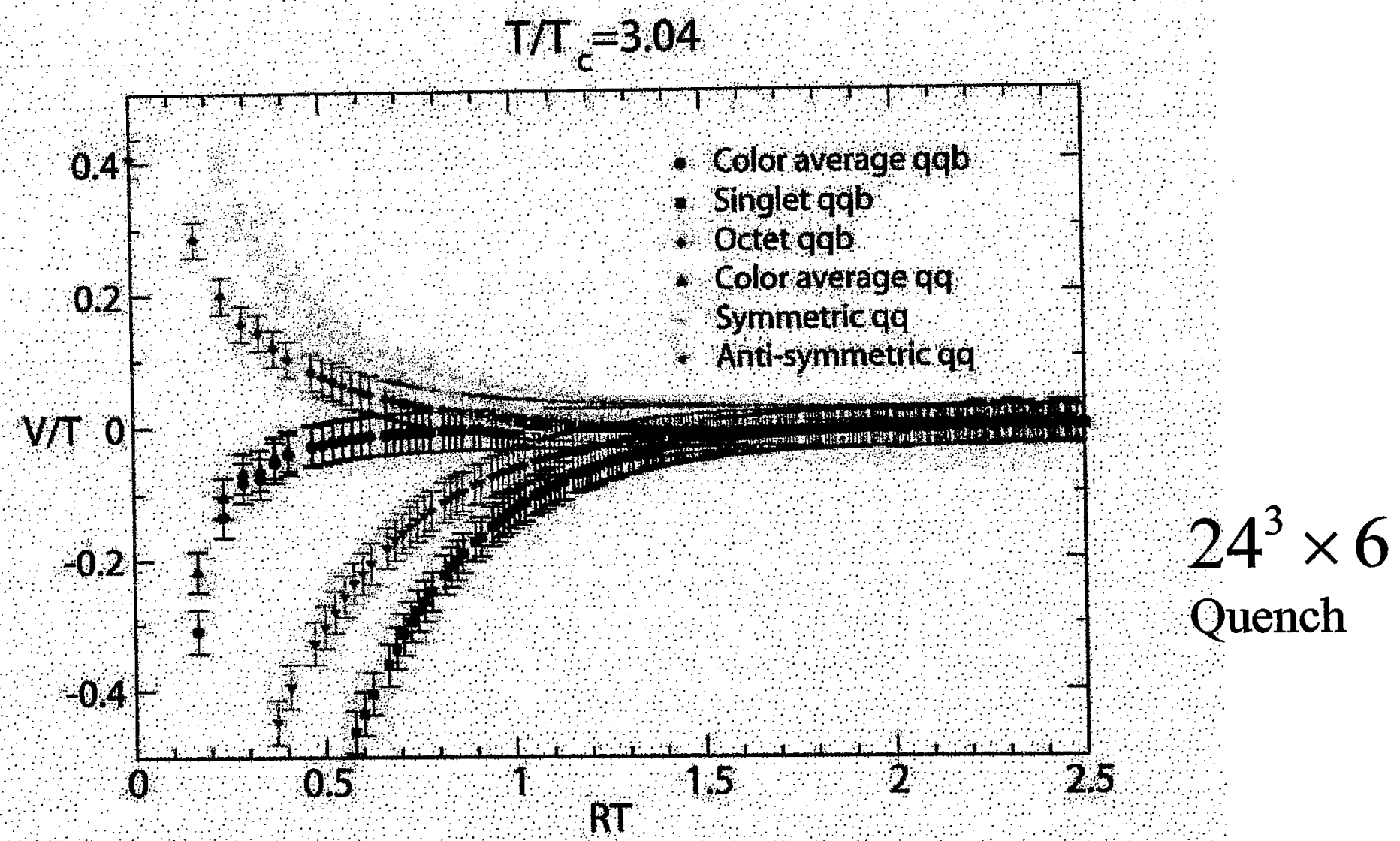




\title{
Lattice QCD at Finite Temperature and Density \\ RIKEN BNL Research Center \\ February 8-12, 2004
}

\author{
REGISTERED PARTIPANTS
}

Name

Masayuki Asakawa

Tom Blum

Barak Bringoltz

Gerald Brown

Michael Cheng

Mike Creutz

Saumen Datta

Shinji Ejiri

Philippe de Forcrand

Zoltan Fodor

Sourendu Gupta

Simon Hands

Urs Heller

Noriyoshi Ishii

Chulwoo Jung

Olaf Kaczmarek

Keijo O. Kajantie

Frithjof Karsch

Sandor Katz

Changhoan Kim

Edwin Laermann

Jonathan Lenaghan

Ludmila Levkova

Shu Li

Huey-Wen Lin

Meifeng Lin

Hideo Matsufuru

Robert Mawhinney

Atsushi Nakamura

Affiliation

E-Mail Address

Kyoto University

University of Connecticut

Tel Aviv University

SUNY, Stony Brook

Columbia University

BNL

Universitaet Bielefeld

Universitaet Bielefeld

ETH Zurich

University of Wuppertal

TIFR

University of Wales Swansea

APS \& BNL

Tokyo Institute of Technology

BNL

University of Bielefeld

University of Helsinki

Bielefeld University

University of Wuppertal

Columbia University

Bielefeld University

University of Virginia

Columbia University

Columbia University

Columbia University

Columbia University

KEK

Columbia University

Hiroshima University yuki@ruby.scphys.kyoto-u.ac.jp

tblum@bnl.gov

barak@viki.tau.ac.il

gerald.brown@stonybrook.edu

michaelc@phys.columbia.edu

creutz@bnl.gov

saumen@physik.uni-bielefeld.de

ejiri@Physik.Uni-Bielefeld.DE

forcrand@phys.ethz.ch

fodor@bodri.elte.hu

sgupta+bn1@theory.tifr.res.in

s.hands@swan.ac.uk

heller@aps.org

ishii@th.phys.titech.ac.jp

chulwoo@quark.phy.bnl.gov

okacz@physik.uni-bielefeld.de

keijo.kajantie@helsinki.fi

karsch@physik.uni-bielefeld.de

katz@bodri.elte.hu

chateau@phys.columbia.edu

edwin@physik.uni-bielefeld.de

j18bf@mail.phys.virginia.edu

ludmila@phys.columbia.edu

lishu@phys.columbia.edu

hwlin@theory1.phys.columbia.edu

mflin@phys.columbia.edu

hideo.matsufuru@kek.jp

rdm@physics.columbia.edu

nakamura@riise.hiroshima-u.ac.jp 


\section{Lattice QCD at Finite Temperature and Density \\ RIKEN BNL Research Center \\ February 8-12, 2004 \\ REGISTERED PARTIPANTS}

Name

Affiliation

E-Mail Address

Shigemi Ohta

Peter Petreczky

Konstantin Petrov

Owe Philipsen

Christian Schmidt

Edward Shuryak

Misha Stephanov

Takanori Sugihara

Benjamin Svetitsky

Derek Teaney

Mikko Vepsalainen

Felix Zantow
BNL / KEK

BNL

BNL

University of Sussex

University of Wuppertal

SUNY, Stony Brook

Univ of IL, Chicago and BNL

BNL

Tel Aviv University

BNL

University of Helsinki

University of Bielefeld ohta@bnl.gov

petreczk@bnl.gov

petrov@bnl.gov

O.Philipsen@sussex.ac.uk

cschmidt@theorie.physik.uni-wuppertal.de

edward.shuryak@sunysb.edu

misha@uic.edu

sugihara@bnl.gov

bqs@julian.tau.ac.il

dteaney@quark.phy.bnl.gov

Mikko.T.Vepsalainen@helsinki.fi

zantow@physik.uni-bielefeld.de 


\section{LATtice QCD AT Finite TEMPERATURE AND DENSITy \\ RIKEN BNL Research Center Workshop \\ Physics Department, Bldg. 510, Large Seminar Room \\ Sunday, Feb. 8 - Thursday, Feb. 12, 2004}

Sunday, February 8, 2004:

BULK THERMODYNAMICS I

09.00-09.45 Continental Breakfast and Registration

09.45-10.00 Welcome N. Samios, M. Creutz, T.Blum, P. Petreczky

10.00-10.50 F. Karsch $\quad$ QCD thermodynamics on lattice

10.50-11.40 R. Mawhinney Lattice QCD thermodynamics with domain wall fermions and anisotropic staggered fermions

11.40-12.30 U.M. Heller Phase diagram of finite temperature QCD with improved staggered fermions

12.30-14.00 Lunch Break

\section{BULK THERMODYNAMICS II}

14.00-15.00 Z. Fodor The phase diagram of $\mathrm{QCD}$ at finite temperature and baryon density

15.00-15.30 Coffee Break

15.30-16.30 C. Schmidt Taylor expansion at non-zero mu and the chiral critical end-point in 3 flavor QCD

16.30-17.30 O. Philipsen $\quad$ QCD thermodynamics from imaginary mu

17:30-18:00 Discussions 
Monday, February 9, 2004:

\section{BULK THERMODYNAMICS III}

09.00-10.00 S. Katz The QCD equation of state at finite $\mathrm{T}$ and $\mathrm{mu}$

10.00-10.30 Coffee Break

10.30-11.30 S. Ejiri QCD thermodynamics at non-zero mu

11.30-12.10 P. de Forcrand $\quad$ QCD at zero baryon density

12.10-14.00 Lunch Break

\section{HEAVY QUARK POTENTIALS}

$\begin{array}{lll}14.00-14.30 & \text { O. Philipsen } & \text { The free energy of static charges at finite temperature } \\ 14.30-15.30 & \text { F. Zantow } & \text { A new look at the QCD potential energy at finite temperature } \\ 15.30-16.00 & \text { Coffee Break } & \\ 16.00-16.40 & \text { O. Kaczmarek } & \begin{array}{l}\text { Heavy quark free energies and the renormalized Polyakov loop in full } \\ \text { QCD }\end{array} \\ 16.40-17.20 & \text { K. Petrov } & \text { Static quark-antiquark potentials with Asqtad improved fermions } \\ 17.20-17.45 & \text { Discussions } & \end{array}$

Tuesday, February 10, 2004:

\section{HADRON PROPERTIES AT T>0 I}

$\begin{array}{lll}09.00-10.00 & \text { M. Asakawa } & \text { Mesonic spectral functions at finite temperature } \\ 10.00-10.30 & \text { Coffee Break } & \\ 10.30-11.30 & \text { S. Datta } & \text { Quarkonium at finite temperature } \\ 11.30-12.10 & \text { N. Ishii } & \begin{array}{l}\text { Properties of the glueball at finite temperature from anisotropic lattice } \\ \text { QCD }\end{array} \\ 12.10-14.00 & \text { Lunch Break } & \end{array}$




\title{
Tuesday, February 10, 2004, continued:
}

\section{HADRON PROPERTIES AT T $>0$ II}

$\begin{array}{lll}14.00-15.00 & \text { E. Laermann } & \text { Light quark meson correlations at high temperature } \\ 15.00-15.30 & \text { Coffee Break } & \\ 15.30-16.30 & \text { H. Matsufuru } & \text { Meson correlators at T>0 in lattice QCD } \\ 16.30-17.30 & \text { S. Gupta } & \text { Temporal and spatial hadron corrrelators in finite temperature QCD } \\ 17.30-18.00 & \text { Discussions } & \end{array}$

\section{Wednesday, February 11, 2004:}

\section{THEORETICAL DEVELOPEMENTS}

\author{
09.00-10.00 S. Hands Lattice simulations at the Fermi surface \\ 10.00-10.40 B. Bringoltz Order from disorder in lattice QCD with baryons \\ 10.40-11.00 Coffee Break \\ 11.00-11.50 E. Shuryak $\quad$ Quark-gluon plasma at strong and very strong coupling \\ 11.50-13.30 Lunch Break \\ 13.30-14.10 J. Lenaghan Deconfining phase transition and matrix models for renormalized \\ Polyakov loops \\ 14.10-14.50 D. Teaney Collective behavior in heavy-ion collisions \\ 14.50-15.30 Coffee Break \\ 15.30-16.30 BNL Physics Colloquium \\ Krishna Rajagopal Unveiling the phases of QCD matter
}

\section{Thursday, February 12, 2004:}

09.00-10.00 K. Kajantie QCD thermodynamics and 3d effective theories

10.00-10.30 Coffee Break

10.30-11.10 M. Vepsalainen Mesonic screening masses at high temperature

11.10-12.00 A. Nakamura Gluon screening masses 


\section{Additional RIKEN BNL Research Center Proceedings:}

Volume 60 - Lattice QCD at Finite Temperature and Density - BNL-

Volume 59 - RHIC Spin Collaboration Meeting XXI, January 2004 - BNL-

Volume 58 - RHIC Spin Collaboration Meeting XX - BNL- 71900-2004

Volume 57 - High pt Physics at RHIC, December 2-6, 2003 - BNL-

Volume 56 - RBRC Scientific Review Committee Meeting - BNL- 71899-2003

Volume 55 - Collective Flow and QGP Properties - BNL-71898-2003

Volume 54 - RHIC Spin Collaboration Meetings XVII, XVIII, XIX - BNL-71751-2003

Volume 53 - Theory Studies for Polarized $p p$ Scattering - BNL-71747-2003

Volume 52 - RIKEN School on QCD "Topics on the Proton" - BNL-71694-2003

Volume 51 - RHIC Spin Collaboration Meetings XV, XVI - BNL-71539-2003

Volume 50 - High Performance Computing with QCDOC and BlueGene - BNL-71147-2003

Volume 49 - RBRC Scientific Review Committee Meeting - BNL-52679

Volume 48 - RHIC Spin Collaboration Meeting XIV - BNL-71300-2003

Volume 47 - RHIC Spin Collaboration Meetings XII, XIII - BNL-71118-2003

Volume 46 - Large-Scale Computations in Nuclear Physics using the QCDOC - BNL-52678

Volume 45 - Summer Program: Current and Future Directions at RHIC - BNL-71035

Volume 44 - RHIC Spin Collaboration Meetings VIII, IX, X, XI - BNL-71117-2003

Volume 43 - RIKEN Winter School - Quark-Gluon Structure of the Nucleon and QCD - BNL-52672

Volume 42 - Baryon Dynamics at RHIC - BNL-52669

Volume 41 - Hadron Structure from Lattice QCD - BNL-52674

Volume 40 - Theory Studies for RHIC-Spin - BNL-52662

Volume 39 - RHIC Spin Collaboration Meeting VII - BNL-52659

Volume 38 - RBRC Scientific Review Committee Meeting - BNL-52649

Volume 37 - RHIC Spin Collaboration Meeting VI (Part 2) - BNL-52660

Volume 36 - RHIC Spin Collaboration Meeting VI - BNL-52642

Volume 35 - RIKEN Winter School - Quarks, Hadrons and Nuclei - QCD Hard Processes and the Nucleon Spin - BNL-52643

Volume 34 - High Energy QCD: Beyond the Pomeron - BNL-52641

Volume 33 - Spin Physics at RHIC in Year-1 and Beyond - BNL-52635

Volume 32 - RHIC Spin Physics V - BNL-52628

Volume 31 - RHIC Spin Physics III \& IV Polarized Partons at High Q^2 Region - BNL-52617

Volume 30 - RBRC Scientific Review Committee Meeting - BNL-52603

Volume 29 - Future Transversity Measurements - BNL-52612

Volume 28 - Equilibrium \& Non-Equilibrium Aspects of Hot, Dense QCD - BNL-52613

Volume 27 - Predictions and Uncertainties for RHIC Spin Physics \& Event Generator for RHIC Spin Physics III - Towards Precision Spin Physics at RHIC - BNL-52596 
Volume 26 - Circum-Pan-Pacific RIKEN Symposium on High Energy Spin Physics - BNL-52588 Volume 25 - RHIC Spin - BNL-52581

Volume 24 - Physics Society of Japan Biannual Meeting Symposium on QCD Physics at RIKEN BNL Research Center - BNL-52578

Volume 23 - Coulomb and Pion-Asymmetry Polarimetry and Hadronic Spin Dependence at RHIC Energies - BNL-52589

Volume 22 - OSCAR II: Predictions for RHIC - BNL-52591

Volume 21 - RBRC Scientific Review Committee Meeting - BNL-52568

Volume 20 - Gauge-Invariant Variables in Gauge Theories - BNL-52590

Volume 19 - Numerical Algorithms at Non-Zero Chemical Potential - BNL-52573

Volume 18 - Event Generator for RHIC Spin Physics - BNL-52571

Volume 17 - Hard Parton Physics in High-Energy Nuclear Collisions - BNL-52574

Volume 16 - RIKEN Winter School - Structure of Hadrons - Introduction to QCD Hard Processes BNL-52569

Volume 15 - QCD Phase Transitions - BNL-52561

Volume 14 - Quantum Fields In and Out of Equilibrium - BNL-52560

Volume 13 - Physics of the 1 Teraflop RIKEN-BNL-Columbia QCD Project First Anniversary Celebration - BNL-66299

Volume 12 - Quarkonium Production in Relativistic Nuclear Collisions - BNL-52559

Volume 11 - Event Generator for RHIC Spin Physics - BNL-66116

Volume 10 - Physics of Polarimetry at RHIC - BNL-65926

Volume 9 - High Density Matter in AGS, SPS and RHIC Collisions - BNL-65762

Volume 8 - Fermion Frontiers in Vector Lattice Gauge Theories - BNL-65634

Volume 7 - RHIC Spin Physics - BNL-65615

Volume 6 - Quarks and Gluons in the Nucleon - BNL-65234

Volume 5 - Color Superconductivity, Instantons and Parity (Non?)-Conservation at High Baryon Density - BNL-65105

Volume 4 - Inauguration Ceremony, September 22 and Non -Equilibrium Many Body Dynamics BNL-64912

Volume 3 - Hadron Spin-Flip at RHIC Energies - BNL-64724

Volume 2 - Perturbative QCD as a Probe of Hadron Structure - BNL-64723

Volume 1 - Open Standards for Cascade Models for RHIC - BNL-64722 
For information please contact:

Ms. Pamela Esposito

RIKEN BNL Research Center

Building 510A

Brookhaven National Laboratory

Upton, NY 11973-5000 USA

Phone: (631) 344-3097

Fax: (631) 344-4067

E-Mail: pesposit@bnl.gov

Homepage: http://www.bnl.gov/riken
Ms. Tammy Heinz

RIKEN BNL Research Center

Building 510A

Brookhaven National Laboratory

Upton, NY 11973-5000 USA

(631) $344-5864$

(631) 344-2562

theinz@bnl.gov 


\section{(9) RIKEN BNL RESEARCH CENTER \\ Lattice QCD at Finite Temperature and Density}

February 8-12, 2004

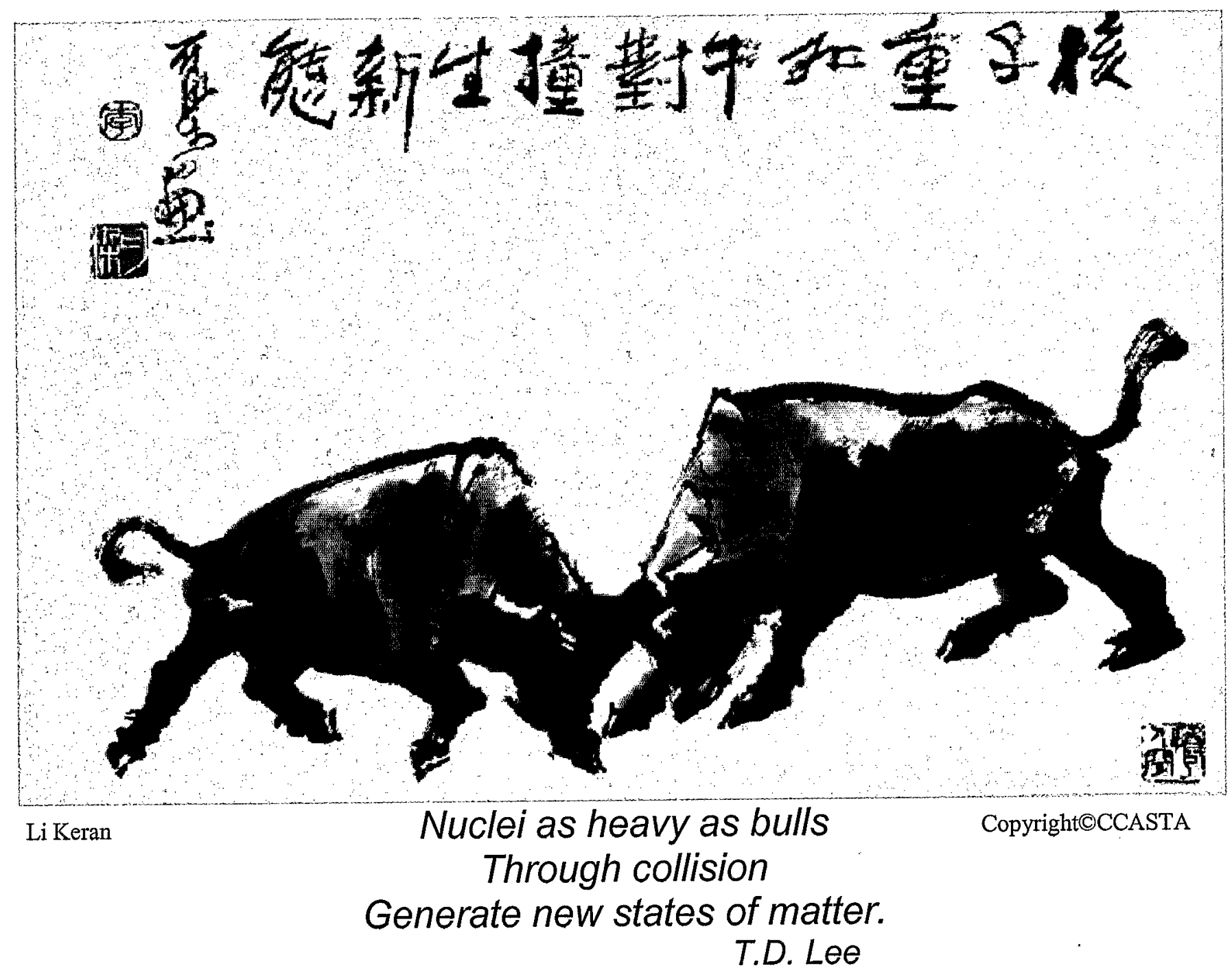

Speakers:
M. Asakawa
B. Bringoltz
S. Datta
P. de Forcrand
S. Ejiri
Z. Fodor
S. Gupta
S. Hands
U. Heller
$\mathrm{N}$. Ishii
O. Kaczmarek
F. Karsch
S. Katz
E. Laermann
J.Lenaghan
K. Kajantie
R. Mawhinney
O. Philipsen
H. Matsufuru
A. Nakamura
K. Petrov
C. Schmidt
B. Svetitsky
M. Vepsalainen
F. Zantow

Organizers: T. Blum, M. Creutz, and P. Petreczky 